\title{
USING QUASI-ELASTIC EVENTS TO MEASURE NEUTRINO OSCILLATIONS WITH MINOS DETECTORS IN THE NUMI NEUTRINO BEAM
}

\author{
A Dissertation \\ by \\ MASAKI WATABE \\ Submitted to the Office of Graduate Studies of \\ Texas A\&M University \\ in partial fulfillment of the requirements for the degree of \\ DOCTOR OF PHILOSOPHY
}

May 2010

Major Subject: Physics 


\title{
USING QUASI-ELASTIC EVENTS TO MEASURE NEUTRINO OSCILLATIONS WITH MINOS DETECTORS IN THE NUMI NEUTRINO BEAM
}

\author{
A Dissertation \\ by \\ MASAKI WATABE
}

\begin{abstract}
Submitted to the Office of Graduate Studies of Texas A\&M University in partial fulfillment of the requirements for the degree of

DOCTOR OF PHILOSOPHY
\end{abstract}

Approved by:

Chair of Committee, Robert C. Webb

Committee Members, Stephen Fulling

Teruki Kamon

David Toback

Head of Department, Edward Fry

May 2010

Major Subject: Physics 


\author{
ABSTRACT \\ Using Quasi-Elastic Events to Measure Neutrino Oscillations \\ with MINOS Detectors in the NuMI Neutrino Beam. (May 2010) \\ Masaki Watabe, B.S., Drexel University; \\ M.S., Texas A\&M University \\ Chair of Advisory Committee: Dr. Robert C. Webb
}

MINOS (Main Injector Neutrino Oscillation Search) experiment has been designed to search for a change in the flavor composition of a beam of muon neutrinos as they travel between the Near Detector at Fermi National Accelerator Laboratory and the Far Detector in the Soudan mine in Minnesota, $735 \mathrm{~km}$ from the target. The MINOS oscillation analysis is mainly performed with the charged current (CC) events and sensitive to constrain high- $\Delta m^{2}$ values. However, the quasi-elastic (QEL) charged current interaction is dominant in the energy region important to access low- $\Delta m^{2}$ values. For further improvement, the QEL oscillation analysis is performed in this dissertation. A data sample based on a total of $2.50 \times 10^{20} \mathrm{POT}$ is used for this analysis. In summary, 55 QEL-like events are observed at the Far detector while $87.06 \pm 13.17$ (syst.) events are expected with null oscillation hypothesis. These data are consistent with $\nu_{\mu}$ disappearance via oscillation with $\Delta m^{2}=2.10 \pm 0.37$ (stat.) \pm 0.24 (syst.) $\mathrm{eV}^{2}$ and the maximal mixing angle. 


\section{ACKNOWLEDGMENTS}

I would like to thank my committee chair, Dr. Webb, my committee members, Dr. Fulling, Dr Kamon, Dr. Toback and Dr. Dutta for their guidance and support throughout the course of this research.

Thanks also to my friends and colleagues and the department faculty and staff for making my time at Texas A\&M University a great experience. I also want to extend my gratitude to the MINOS collaborators and Fermi National Accelerator Laboratory, which provided the survey instrument and service work to maintain running the MINOS experiment. This work has been supported in part by the U.S. Department of Energy through grant DE-FG02-95ER40917.

Finally, thanks to my family for their encouragement and support throughout my time as a student. 


\section{TABLE OF CONTENTS}

CHAPTER

MASSIVE NEUTRINOS . . . . . . . . . . . . . . . . . . 4

A. Fundamental Properties . . . . . . . . . . . 5

1. Dirac and Majorana Particles . . . . . . . . . 5

2. Lepton Number Violation . . . . . . . . . . . . 6

3. Neutrino Mixing . . . . . . . . . . . . . . . . 6

B. Neutrino Mass Measurement . . . . . . . . . . . . . . 9

1. Solar Neutrinos . . . . . . . . . . . . . . . . . 12

2. Atmospheric Neutrinos . . . . . . . . . . . . . 16

3. $\nu_{e}$ Appearance . . . . . . . . . . . . . . . 19

C. Light Neutrino Mass Generation Mechanism . . . . . . . . 24

1. Seesaw Mechanism . . . . . . . . . . . . . 24

2. Exotic Neutrino Search . . . . . . . . . . . . 26

III THE NUMI-MINOS EXPERIMENT . . . . . . . . . . . 29

A. NuMI Neutrino Beamline . . . . . . . . . . . . . . . . 29

B. The MINOS Detectors . . . . . . . . . . . . . 33

C. Event Reconstruction . . . . . . . . . . . . . . . 36

D. Detector Calibration ............ 37

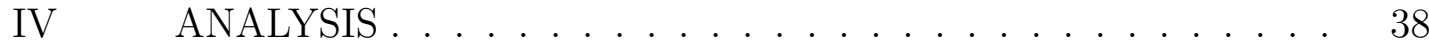

A. Motivation .................. . . 38

B. The Near Detector Analysis . . . . . . . . . . . . . 41

1. Model Adjustment : QEL Cross Sections . . . . . . 41

2. QEL Selection : QEL Kinematic Correction . . . . . . 46

3. Low- vs High- $Q^{2}$ Selection . . . . . . . . . . . . 54

4. Event Distribution/Beam Stability . . . . . . . . . 57

5. Near Detector Fit . . . . . . . . . . . . . . . . 60

C. The Far Detector Analysis . . . . . . . . . . . . . . . . 72

1. QEL Selection : QEL Kinematic Correction . . . . . . 72

2. Event Distribution/Beam Stability . . . . . . . . 76

3. Oscillation Fit . . . . . . . . . . . . . . 76 
CHAPTER

REFERENCES . . . . . . . . . . . . . . . . . . 87

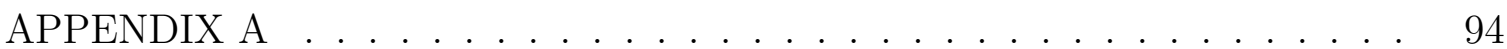

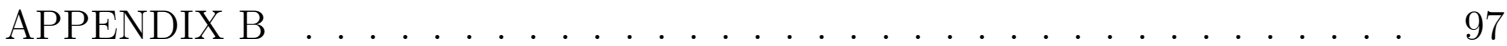

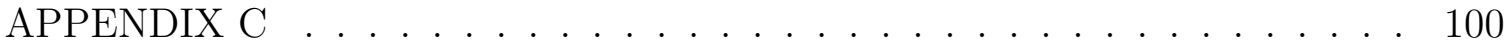

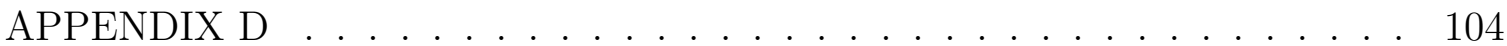

APPENDIX E . . . . . . . . . . . . . . . . . . . . . 127

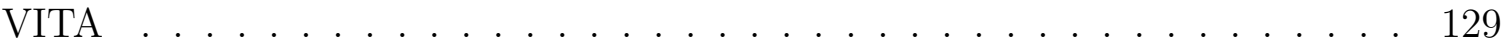




\section{LIST OF TABLES}

TABLE

I Lepton number conservation and violation. $L, L_{e}$ and $L_{\mu}$ are denoted as total, electron and muon lepton number. (Left) The lepton number is conserved for the SM-like muon decay. (Right) The electron and muon lepton numbers are violated in one of the possible muon decay processes if neutrino is massive and Majorana

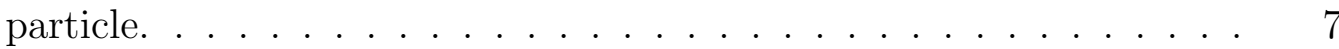

II Solar neutrino flux. Solar neutrino flux obtained from various experiments. SNO experiment is sensitive to measure the flux for ES, CC and NC interaction channels. The other experiments are only sensitive to the single interaction channel as indicated. The flux ratio means the ratio of the observed to expected SSM flux. . . .

III Systematic shift for oscillation parameters. The systematic shifts for the fitted parameters are calculated with MC "fake" (or mock) data samples for $\Delta m^{2}=2.102 \times 10^{-3} \mathrm{eV}^{2}$ and $\sin ^{2}(2 \theta)=1.00$. Systematic shifts for $\Delta m^{2}, \sin ^{2}(2 \theta)$ and the expected event number respect to the $10^{20}$ POT counting are shown in 3rd, 4th and

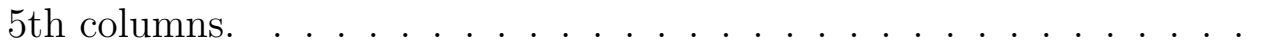

IV Near Detector mock data fit results. Four parameters are included in the Near Detector shape-only and shape+rate fits. The Near Detector mock data set is generated with the detector and cross section parameters and SKZP reweighted beam spectrum. The minimum values of the $\chi^{2}$ are less than degree of freedom. . . . . . 106

V Near Detector data fit results. Four parameter fits with NonQE scale are shown in 2 nd and 4th columns. 3rd and 5th columns show four parameter fit results with the $M^{R E S}$-scale. The minimum values of the $\chi^{2}$ are larger than degree of freedom. . . . . . . . 114

VI Far Detector oscillation mock data fit result. FD oscillation fit result for $65 \times 10^{20}$ pot mock data set using the shape+rate Near Detector fit result. . . . . . . . . . . . . . . . . . . . . 127 


\section{LIST OF FIGURES}

FIGURE

$1 \quad$ Neutrino mass hierarchy. $\nu_{3}$ is the lightest neutrino in the inverted hierarchy. $\nu_{1}$ is the lightest neutrino in the normal hierarchy. . . . .

Solar neutrino flux. The SSM flux is shown as a function of neutrino energy for different nuclear fusion channels [38].

Result from KamLAND experiment. (Left) $L / E$ spectrum shows that neutrino is maximally oscillated around $L / E \sim 50 \mathrm{~m} / \mathrm{MeV}$. (Right) Black line shows the result from solar neutrino data. The color contour shows the fitting result from KamLAND data for the total exposure of $2.44 \times 10^{32}$ proton-yr (2881 ton-yr). These figures are referenced from $[41] . \ldots \ldots \ldots$

4 SK result of atmospheric neutrino. (Left) SK result of atmospheric neutrino $L / E$ spectrum. The best-fit expectations are shown for neutrino oscillation (solid line) and decay (dashed line) and decoherence (dotted line). (Right) 68\%, 90\% and 99\% C.L. allowed oscillation parameter regions. These figures are referenced from $[42] \ldots \ldots \ldots \ldots \ldots \ldots \ldots$

$5 \quad$ Neutrino energy distribution and ratio from MINOS experiment. Expected and observed CC energy spectrum at the Far Detector is shown at left. The oscillated depletion is shown around $3 \mathrm{GeV}$. The ratio of data to $\mathrm{MC}$ is shown at right. These figures are referenced from $[9] . \ldots \ldots \ldots \ldots$

$6 \quad$ Oscillation contour from MINOS experiment. The oscillation contour of $\Delta m^{2}$ vs $\sin ^{2}(2 \theta)$ is shown in the figure. This figure is referenced from $[9] . \ldots \ldots \ldots \ldots \ldots$ 
$7 \quad$ Limitation of MINOS experiment. Left and right figures show the sensitivity for $\Delta m^{2}$ and the mixing angle measurements as a function of the POT exposure for the low energy beam configuration. $68 \%$ and $90 \%$ confidence level regions are for black and red line. The systematic error is added for the dashed line. Red straight and shaded region are for SK 90\% confidence limit. These figures are referenced from $[46] . \ldots \ldots \ldots \ldots \ldots$

8 NuMI off-axis beam spectrum. (Left) Red line shows that the the relativistic relation between pion and neutrino on the beam axis. The neutrino is less dependent to pion energy for small angle off the beam axis. (Right) Black points show the neutrino energy spectrum for the NuMI medium beam energy configuration onaxis. The spectrum is shifted near the maximally oscillated position if the off-axis angle is $14 \mathrm{mrad}$ and shown in red histogram. These figures are referenced from $[49] . \ldots$. . . . . . . . . . .

$9 \quad \theta_{13}$ sensitivity contour in the $\mathrm{NO} \nu \mathrm{A}$ experiment. $\sin ^{2}\left(2 \theta_{13}\right)$ sensitivity contour for $700 \mathrm{~kW}$ beam power are shown in the top figure as a function of the CP phase in solid line. The figure is referenced from $[50] . \ldots \ldots \ldots \ldots \ldots \ldots \ldots$

10 Mass ordering sensitivity in the $\mathrm{NO} \nu \mathrm{A}$ experiment. Normal and inverted mass hierarchy are in red and blue color. Mass ordering sensitivity contours for $700 \mathrm{~kW}$ beam power are shown as a function of the CP phase in dash-dot line. The left and right bottom figures are for normal and inverted hierarchy. These figures are referenced from $[50] \ldots \ldots \ldots \ldots \ldots$

11 Mass heirarchy. Vertical and horizontal axes show the mass scale in $\mathrm{eV}$ and 3 generations respectively. u-type and d-type quarks and charged leptons are marked in black, red and green points. Neutrinos and top quark are extremely light and heavy comparing to the other fundamental particles. Ranges for neutrino mass is shown in blue lines. . . . . . . . . . . . . . . . . 
FIGURE

12 Result from MiniBooNE experiment. (Left) Top figure shows the neutrino energy spectrum in MB. Bottom one is the ratio figure of the expected to observed spectra. MB data above $475 \mathrm{MeV}$ is consistent with no oscillation. (Right) $90 \% \mathrm{MB}$ exclusion region is shown with dashed and solid lines. LSND signal filled in blue is clearly excluded. Those figures are referenced from [58]. . . . . . .

13 Overview of MINOS experiment. Overview of MINOS experiment $[44,45] \ldots \ldots \ldots \ldots \ldots \ldots$

14 Schematic view of the NuMI beamline. Protons from the MI enter from the left and the produced neutrinos exit to the right $[44,45]$

15 NuMI beam configurations. The Near Detector MC spectra for low, medium and high energy beam configurations. If the distance between the target position and first horn is increased, the peak energy position is shifted to higher energy. . . . . . . . . . .

16 Schematic view for one layer of scintillator plane in the detector [44]. . . . . . . . . . . . . . . . . .

17 Photograph of the Near Detector. The Near Detector [44, 45] located $104 \mathrm{~m}$ underground and consists of 282 irregular $4 \times 6 \mathrm{~m}^{2}$ octagonal steel and 153 scintillator planes read out at via a WLS fiber to one pixel of a Hamamatsu Multiplex M64 PMT and the Fast QIE electronics system that is capable of continuously recording signals without dead time throughout the $\sim 10 \mu$ s beam spill. A toroidal magnetic field is a toroidal field produced by a running a current of $185 \mathrm{kA}$ through the center of the detector producing an average value of $1.2 \mathrm{~T} \ldots \ldots \ldots \ldots$

18 Photograph of the Far Detector. The Far Detector [44, 45] is located $705 \mathrm{~m}$ beneath the surface and consists of 484 octagonal $8 \mathrm{~m}$ wide steel and scintillator planes read out at both ends via Hamamatsu Multiplex M16 PMTs and the VA electronics systems. The $40 \mathrm{kA}$ current carried by the coil running through the center of the detector induces the magnetic field of $1.17 \mathrm{~T}$. Veto shields are installed on the top of the detector to remove cosmic muon events. . 
FIGURE

19 2-dimensional views of the $\mathrm{CC}$ and $\mathrm{NC} \nu_{\mu}$ interactions. The 2dimensional views of the simulated events for the CC and $\mathrm{NC} \nu_{\mu}$ interactions in the Near Detector. The shaded rectangular boxes indicate relative energy deposited in the strip. These figures are referenced from $[44,45,82] . \ldots \ldots \ldots \ldots$

20 Results from K2K and MINOS experiments. (Left) Energy spectrum is configured below $1 \mathrm{GeV}$ for $\mathrm{K} 2 \mathrm{~K}$ experiment $[71,51]$. (Right) MINOS energy is peaked out of the maximal position [9]. . .

$21 \quad$ Neutrino cross section. Neutrino cross section for QEL (red), RES (blue) and DIS (green) interactions shows as a function of neutrino energy. The figure is referenced from $[72] \ldots \ldots \ldots \ldots$

22 CC-like event distributions. CC-like event distributions and the ratio spectra of MC to data are shown for the 1.27 and the $1.47 \times$ $10^{20}$ POT data and MC sets from the low energy beam configuration $(\mathrm{LE}-010,185 \mathrm{kA})$. Blue and red dashed lines are for the nominal and SKZP reweighted MC. The $E_{\nu}^{C C}, Q^{2}$ and $y$ distributions and data/MC ratios are shown in the top, middle and bottom figures. . . . . . . . . . . . . .

23 Effect of changing $M_{A}^{Q E L}$-scae for free nucleon QEL interaction cross section. Effect of changing $M_{A}^{Q E L}$ scale for free nucleon QEL interaction cross section. Shape and normalization of the $Q^{2}$ distribution are influenced by changing the $M_{A}^{Q E L}$ scale. Left and right figures are scaled by the POT counting and the area of $M_{A}=1.00 \mathrm{GeV}$. These curves are correspond to mono-energetic neutrinos at $1 \mathrm{GeV}$ and ignore nuclear effects. The high $Q^{2}$ cutoff is purely kinematic. These figures are referenced from $[78] . \quad \ldots .$. .

24 Near Detector POT distribution. POT and integrated POT distributions are shown as a function of time or run number. . . . . . .

25 Near Detector CC-like event distributions. (Left) PID (particle identification) is the parameter to discriminate between CC- and NC-like events. MC is normalized to the data event number. (Right) Efficiency and purity of the CC-like event selection are shown in blue and red lines. . . . . . . . . . . . . . . . . . 
FIGURE

26 Transformation of the internal shower spread. Shower is a point and track in minimum and maximum limits. . . . . . . . . . . . . . . 50

27 Shower energy distribution. First kinematic cut of shower energy less than $1 \mathrm{GeV}$ is indicated in pink line. Red histogram shows true QEL event distribution. . . . . . . . . . . . . . .

28 Schematic view of QEL neutrino interaction. Incident neutrino, outgoing muon and proton are shown in black, red and blue arrows. .

29 Proton's angular difference. Schematic view of angular differences is shown in the top figure. Incident neutrino, outgoing muon and proton are shown in black, red and blue. The angles converted by QE and CCQE energy spectra are shown in light and dark blue. . . .

30 Proton's angular difference. The angular difference between QE and CCQE spectra is used to select QEL-events. $\delta \theta$ distributions cuts are shown in the left and right figures. A shower energy cut is applied for the right figure. . . . . . . . . . . . . . .

31 The low- $Q^{2}$ selection is used for QEL event selection. Purity and efficiency are shown in the top two figures as a function of neutrino energy and momentum transfer. The $E^{\nu}$ and $Q^{2}$ distributions and data/MC ratios are shown in the middle and bottom two figures. $\mathrm{QE}$ and CCQE energy reconstruction are indicated in red and blue. .

32 The high- $Q^{2}$ selection is used for QEL event selection. Purity and efficiency are shown in the top two figures as a function of neutrino energy and momentum transfer. The $E^{\nu}$ and $Q^{2}$ distributions and data/MC ratios are shown in the middle and bottom two figures. QE and CCQE energy reconstruction are indicated in red and blue. . 56

33 Near Detector event distribution. $r^{2}$ - and $z$-vertex distributions are shown in top and middle figures. They are flat over the detector. The bottom figure shows the distributions of the track angles relative to the neutrino beam direction. . . . . . . . . . . . . 58 
34 Near Detector neutrino energy distribution taken during monthly period. The CC- and QEL-like event distributions measured during the monthly period are shown in left and right figures. The distributions are normalized to POT and only data obtained in the LE-10/185kA configuration is included. . . . . . . . . . . .

35 Beam stability. The event number respect to the POT counting is shown as a function of time or run number. . . . . . . . . . . . .

$36 E_{\nu}$ and $Q^{2}$ distributions of the Near Detector fit to the data. Blue and red lines are for the nominal and best fit spectra. . . . . . . .

$37 \pm 0.02$ shifts of $E_{\mu}$-scale for the $E_{\nu}$ and $Q^{2}$ distributions and data $/ \mathrm{MC}$ ratios. . . . . . . . . . . . . . . .

$38 \pm 0.10$ shifts of $E_{s h w}$-scale for the $E_{\nu}$ and $Q^{2}$ distributions and data $/$ MC ratios. . . . . . . . . . . . . . . . .

$39 \pm 0.050 \mathrm{GeV}$ shifts of $E_{\text {shw }}$-offset for the $E_{\nu}$ and $Q^{2}$ distributions and data/MC ratios. . . . . . . . . . . . . . . .

$40 \pm 0.15$ shifts of $M_{A}^{Q E L}$-scale for the $E_{\nu}$ and $Q^{2}$ distributions and data/MC ratios. . . . . . . . . . . . . . . 68

$41 \pm 0.15$ shifts of $M_{A}^{R E S}$-scale for the $E_{\nu}$ and $Q^{2}$ distributions and data $/ \mathrm{MC}$ ratios. . . . . . . . . . . . . . . .

$42 \pm 1 \sigma$ shifts of $M_{A}^{R E S}$-scale and RES/DIS-transition scale for the $Q^{2}$ and $E_{\nu}$ distributions and data/MC ratios. . . . . . . .

43 Pauli blocking suppression effects. Differential neutrino-nucleon cross section is shown as a function of $Q^{2}$. Low- $Q^{2}$ values are largely influenced by the Pauli-Suppression effects in the RFG mode, compared to the free nucleon prediction. The figure is referenced from $[78] . \ldots \ldots \ldots \ldots \ldots$

$44 \quad$ POT and integrated POT distributions. POT and integrated POT distributions are shown as a function of time or run number. Low energy beam was not operation during the flat period. . . . . . . 
45 Far Detector CC-like event distributions. (Left) PID(particle identification) is the parameter used to discriminate between CC- and NC-like events. The MC data is normalized to the data event number. (Right) Efficiency and purity of the CC-like event selection are shown in blue and red lines respectively. . . . . . . . . .

46 Far Detector QEL-like event cuts. (Left) Shower energy distribution is shown with only initial cuts. The pink line is for the shower energy cut at $1 \mathrm{GeV}$. (Right) $\delta \theta$ distribution with the shower energy cut. The pink line is for the angle cut at $3^{\circ} . \mathrm{MC}$ is normalized to the data event number for the shower and angle figures.

47 Far Detector QEL-like event cuts. Purity and efficiency as a function of the energy are shown in red and blue lines respectively. . . . .

$48 \quad$ Far Detector event distributions. $r^{2}$ - and $z$-vertex distributions are shown in top and middle figures. The left bottom figure shows the distributions of the track angles relative to the neutrino beam direction. MC distributions are normalized to the data event number.

49 Event timing distribution. The event timing relative to the predicted beam spill at Far Detector are distributed within $50 \mu \mathrm{s}$ timing window. . . . . . . . . . . . . . .

50 Beam stability. The CC- and QEL-like event number respect to the POT counting is shown as a function of time or run number. .

51 Far Detector 2D oscillation fit result to the data. Oscillation Analysis result for $2.50 \times 10^{20}$ pot data set, using the Near Detector fit result. Red line is for the best fit oscillated spectrum. Black and blue lines are for the best fit and nominal expected spectra. . .

52 Far Detector 1D oscillation fit result to the data. Oscillation Analysis result for $2.50 \times 10^{20}$ pot data set, using the Near Detector fit result. Mixing angle is fixed to maximal. Red line is for the best fit oscillated spectrum. Black and blue lines are for the best fit and nominal expected spectra. The $\Delta \chi^{2}$ curve of the $\Delta m^{2}$ parameter respect to the best fit $\Delta m^{2}$ is shown in the bottom figure. 
FIGURE

53 Comparison of the QEL oscillation contour. The $68 \%$ and $90 \%$ C.L. allowed regions for the oscillation parameters are shown in solid and dashed blue lines. Systematic errors are not included in the contour for the oscillation fit to the QEL data sample. Also shown are contours from previous experiments $[42,43,71,51]$ and MINOS earlier results $[9,45] . \ldots \ldots . \ldots . \ldots 86$

54 The transformation of the internal shower spread. Shower is a point and track in minimum and maximum limits. . . . . . . . . . .

55 Example for shower density function. Set $f\left(p_{0}\right)=\rho_{0}=1.0$ for fixed shower energy. Left and right figures show the $\rho(r)$ and $f(p)$ when varying the M-scale. Changing the $M$-scale is sensitive to change the function width respect to position and momentum. Applying the $|f(p)|^{2}$ is expected to change the shower energy and

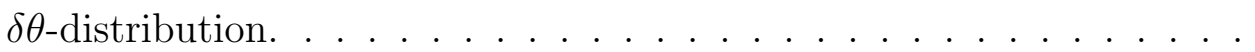

$56 \quad$ The $E_{\nu}^{Q E L}$ and $Q^{2}$ distributions of the shape-only fit to the mock data. Blue and red lines show nominal and best fit spectra. . . . . . 107

57 The parameter $\Delta \chi^{2}$ curves of the shape-only fit to the mock data. . 108

58 The parameter correlations of the shape-only fit to the mock data. . 109

59 The $E_{\nu}^{Q E L}$ and $Q^{2}$ distributions of the shape+rate fit to the mock data. Blue and red lines show nominal and best fit spectra. . . . . . 110

60 The parameter $\Delta \chi^{2}$ curves of the shape+rate fit to the mock data. . 111

61 The parameter correlations of the shape+rate fit to the mock data. 112

62 Fit result (I) : the $E_{\nu}$ and $Q^{2}$ distributions and data/MC ratios of the shape-only fit to the data. Blue and red lines show nominal and best fit spectra. . . . . . . . . . . . . . . . . . 115

$63 \quad$ Fit result (I) : the parameter $\Delta \chi^{2}$ curves of the shape-only fit to the data. . . . . . . . . . . . . . . . . . . 116

64 Fit result (I) : the parameter correlation for the shape-only fit to the data. 
65 Fit result (III) : the $E_{\nu}$ and $Q^{2}$ distributions and data/MC ratios of the shape+rate fit to the data. Blue and red lines are for the nominal and best fit spectra. . . . . . . . . . . . . . . . . . . 118

66 Fit result (III) : the parameter $\Delta \chi^{2}$ curves of the shape+rate fit to the data.

67 Fit result (III) : the parameter correlation for the shape+rate fit to the data.

68 Fit result (II) : the $E_{\nu}$ and $Q^{2}$ distributions and data/MC ratios of the shape-only fit to the data. Blue and red lines are for the nominal and best fit spectra. . . . . . . . . . . . . . . . . . . . . . 121

$69 \quad$ Fit result (II) : the parameter $\Delta \chi^{2}$ curves of the shape-only fit to the data. . . . . . . . . . . . . . . . . . . 122

70 Fit result (II) : the parameter correlation for the shape-only fit to the data. . . . . . . . . . . . . . . . . . . . 123

71 Fit result (IV) : the $E_{\nu}$ and $Q^{2}$ distributions and data/MC ratios of the shape+rate fit to the data. Blue and red lines are for the nominal and best fit spectra. . . . . . . . . . . . . . .

$72 \quad$ Fit result (IV) : the parameter $\Delta \chi^{2}$ curves of the shape+rate fit to the data.

73 Fit result (IV) : the parameter correlation for the shape+rate fit to the data.

74 The oscillation contour, $E_{\nu}$ distribution and data/MC ratio, and $\Delta \chi^{2}$ curves of the oscillation fit to the mock data. The oscillation contour of the oscillation fit to the mock data is shown in the top figure. Pink point indicates true values of oscillation parameters. The $E_{\nu}$ distribution and Data/MC ratio of the oscillation fit to the mock data are shown in the middle left and right figures. The $\Delta \chi^{2}$ curves for the $\Delta m^{2}$ and mixing angle are shown in the bottom left and right figures. . . . . . . . . . . . . . . . . . . . . 128 


\section{CHAPTER I}

\section{INTRODUCTION}

Discovering a Grand Unified Theory (GUT) is one of the ultimate goals of particle physics to unify three fundamental forces (electromagnetic, strong and weak) into single force field. The current best theory of particle physics is well described by the Standard Model (SM). It is a gauge theory of quarks and leptons mediated by the strong and electroweak forces with the gauge group of $\mathrm{SU}(3)_{\mathrm{C}} \times \mathrm{SU}(2)_{\mathrm{L}} \times \mathrm{U}(1)_{\mathrm{Y}}$. The strong interaction is a nuclear theory of the strong force mediated through gluons among quarks to form protons and neutrons. It is governed by color symmetry of $\mathrm{SU}(3)_{\mathrm{C}}$. Similarly the electroweak interaction is a unified theory of weak force and electromagnetism mediated through $W^{ \pm}, Z^{0}$ and $\gamma$ gauge bosons. Electroweak unification occurs at a scale of $100 \mathrm{GeV}$ under the Higgs mechanism that spontaneously breaks the symmetry of $\mathrm{SU}(2)_{\mathrm{L}} \times \mathrm{U}(1)_{\mathrm{Y}}$ group into $U(1)_{Q=Y / 2+I_{3}}$ group, where $Y$ and $I_{3}$ are weak hypercharge and isospin generators respectively. The SM describes the nature of low energy physics very well, but it is only an approximate theory of the GUT in a limit of zero neutrino mass and proton's infinite life time. The primary interest in GUT theories is to search for the physical processes that violate the assumptions made in the SM.

Baryon and lepton numbers are the conserved quantities in the SM. They are preserved under the assumptions that a proton is stable to radioactive decay and a neutrino has no mass. However, GUTs allow the physical processes that violate either one or both of these quantum numbers. Grand unification is estimated to

The journal model is Physical Review Letters. 
occur in a scale of $10^{16} \mathrm{GeV}$ under the mechanism that spontaneously breaks the grand unified group $G$ into the SM gauge group. ${ }^{1}$ The grand unified force is mediated through extremely heavy gauge $X$ and $Y$ bosons that transforms quarks into leptons. Exchanging those heavy bosons can violate those quantum numbers. For example, the minimal SU(5) GUT model predicts the lifetime of the proton when it decays into $e^{+} \pi^{0}$ to be less than $10^{32}$ yrs if a quark transforms into a positron mediated by $X$ gauge boson. A proton is the lightest baryon and can not decay into lighter baryons. It carries +1 baryon number, but the positron and neutral pions have no baryon number. Thus total baryon number is not conserved in this decay mode. At this time, no evidence of proton decay has been found in Super-Kamiokande experiment $[1,2,3]$. The current lower limit is at $10^{33}$ yrs at $90 \%$ C.L.

In 1972, M. Kobayashi and T. Maskawa were inspired by N. Cabibbo's theory of light quark mixing and proposed new theory of quark decay mixing with three generations of six quarks $[4,5,6]$. In their framework, $\mathrm{CP}$ violation appears quite naturally and the mixture of flavor and mass quantum states of quarks are represented in the $3 \times 3$ matrix known as CKM (Cabibbo-Kobayashi-Maskawa) mixing matrix. This mixture is only present when quarks are massive. Details of the CKM framework were confirmed at BaBar and Belle experiments in the USA and Japan $[7,8]$. Unlike the quark sector, the $\mathrm{CP}$ violation disappears in the lepton sector and the flavor and mass quantum states of leptons are identical under the assumption that a neutrino has no mass. Leptonic mixing matrix is just unity and a charged lepton can only couple to its own neutrino. However, when the neutrino has mass, the GUTs should allow the leptonic quantum mixture and $\mathrm{CP}$ violation into a new quantum framework.

${ }^{1}$ The GUT scale is 14 orders of magnitude larger than the electroweak unification scale. It is known as gauge hierarchy problem. Supersymmetry is one of the popular theories to resolve the problem. 
The SM is only an approximate theory of the GUT if a neutrino has mass and the baryon and lepton numbers are not conserved. Current particle physics studies are motivated by searching for new physics beyond these limits. In 1998, the SuperKamiokande collaboration in Japan discovered strong evidence of neutrino mass in atmospheric neutrino oscillation sector, which implies that the quantum mixing and $\mathrm{CP}$ violation are present in the lepton sector. Currently many neutrino experiments are operating in the world. They are designed to measure absolute and relative scales of neutrino mass and all matrix elements of lepton mixing. The NuMI-MINOS experiment is one of the neutrino oscillation experiments at Fermi Accelerator Laboratory (Fermilab) in the USA and has been specially designed to measure relative neutrino mass difference and mixing in the atmospheric neutrino sector. The first MINOS oscillation analysis was performed using charged current (CC) events [9] and sensitive to constrain high- $\Delta m^{2}$ values. However, the quasi-elastic (QEL) charged current interaction is dominant in the energy region important to access low- $\Delta m^{2}$ values. For further improvement, the QEL oscillation analysis is presented in this dissertation. 


\section{CHAPTER II}

\section{MASSIVE NEUTRINOS}

The SM has been extremely successful in explaining various low energy processes involving neutrino interactions. The SM is basically governed by the conservation of the left and right handed helicity states for the charged leptons $(e, \mu, \tau)$ and the quarks $(u, d, s, c, t, b)$. The helicity states are reversible by changing frames of reference if particles have mass. Left handed particles can convert into right handed ones by changing the frame of reference and vice versa. If particles have mass, then the solutions of Dirac equation are mixed with right and left handed functions. For example, assume that an electron moves along the $+z$-direction in our frame of reference. The z-component of its spin is $-1 / 2$. Thus, its momentum and spin are anti-parallel so that the electron is denoted as left handed (LH). However, the electron moves in the $-\mathrm{z}$-direction if the observers run faster than the electron in the z-direction. The momentum and spin are parallel hence the ones in the faster frame observe the right handed electron $(\mathrm{RH})$. Therefore, the helicity is reversible if the particle is massive. On the other hand, if particles are massless, then they can only travel at the speed of light and the helicity states are not reversible. Only left handed helicity state of the neutrino would be present, which means that the helicity states for neutrinos are purely left handed when they are massless. Conversely the helicity of anti-neutrinos are purely right handed as confirmed by G. Backenstoss and M. Bardon [10, 11]. Thus the nature of low energy scale is well described in the SM by taking a relativistic limit for neutrino mass. 


\section{A. Fundamental Properties}

Introducing a massive neutrino is theoretically one of the simplest ways to extend the SM [12]. It doesn't require any theoretical assumptions such as supersymmetry, extra dimensions etc. How is it possible for massive neutrino to fit into a bigger picture? Assuming a massive neutrino, three fundamental properties instantly appear and are discussed below.

\section{Dirac and Majorana Particles}

The helicity is reversible for a massive particle only if electric charge is conserved under Lorentz transformation. If the LH particle is electrically charged, then it is possible to distinguish between particle and anti-particle under the transformation.

Such particles are called Dirac particles. For example, LH electron can be boosted into $\mathrm{RH}$ electron or anti-electron. It is the $\mathrm{RH}$ electron, not anti-electron. One can find out either one of electron or anti-electron, due to the conservation of electric charge. If there is no conserved quantum number to distinguish between neutrino and anti-neutrino, then neutrino is identical to its own anti-neutrino or a so called Majorana neutrino $[13,14,12]$. For example, the neutral pion is a Majorana particle. Charged pions, $\pi^{+}$and $\pi^{-}$are complex scaler fields that these fields are reversible under the charge conjugation operator. It is possible to distinguish between them, but the neutral pion is only formed with a real scaler field, no complex terms. The field is unchanged under the charge conjugation operator. Therefore, neutral pion is identical to its own anti neutral pion. 


\section{Lepton Number Violation}

Lepton number is one of the conserved quantities in the SM. It is preserved under the assumption that a neutrino is a massless and a Dirac particle. On the other hand, it is violated if a neutrino is massive and a Majorana particle. Neutrinoless double beta decay is one of the nuclear processes that violates the total lepton number and related to Majorana nature of neutrinos. Details are discussed in Ref. [12, 15, 16, 17, $18,19,20,21]$. As an example, muon decay is described in this section. SM-like muon decay can be written in the form of $\mu^{-} \rightarrow e^{-}+\overline{\nu_{e}}+\nu_{\mu}$. Lepton number is 1 for muon, electron and neutrino. Anti-neutrinos carry -1 lepton number. Thus the total lepton number is conserved before and after weak interaction and shown at the left Table I. Total electron and muon numbers are conserved as well. However, the lepton number conservation is violated if a neutrino is massive and a Majorana particle. The SM-like muon decay process can be rewritten in the form of $\mu^{-} \rightarrow e^{-}+\nu_{e}+\overline{\nu_{\mu}}$ hence the muon and electron neutrinos are identical to their own anti-neutrinos if they are Majorana particles. The possible muon decay process is shown at the right Table I. The total lepton number is clearly conserved, but the electron and muon lepton numbers are violated in this process. The electron lepton number is +1 for electron and electron neutrino, and is not defined for muon and muon neutrino. The total electron number is 0 before the weak interaction, but it is 2 after the interaction. The electron number is not only violated in the process, but so is the muon number.

\section{Neutrino Mixing}

The weak force between quarks and leptons is described as the flow of the conserved weak charge and can be carried by a charged and neutral currents (CC and NC) exchanging the $W^{ \pm}$and $Z^{0}$ intermediated gauge bosons. The weak interaction 
Table I: Lepton number conservation and violation. $L, L_{e}$ and $L_{\mu}$ are denoted as total, electron and muon lepton number. (Left) The lepton number is conserved for the SM-like muon decay. (Right) The electron and muon lepton numbers are violated in one of the possible muon decay processes if neutrino is massive and Majorana particle.

\section{Allowed muon decay process}

\begin{tabular}{|c||ccccc|}
\hline & $\mu^{-}$ & $\rightarrow$ & $e^{-}$ & $\overline{\nu_{e}}$ & $\nu_{\mu}$ \\
\hline$L$ & +1 & & +1 & -1 & +1 \\
\hline$L_{e}$ & 0 & $=$ & +1 & -1 & 0 \\
\hline$L_{\mu}$ & +1 & $=$ & 0 & 0 & +1 \\
\hline
\end{tabular}

\section{Possible muon decay process}

\begin{tabular}{|c||ccccc|}
\hline & $\mu^{-}$ & $\rightarrow$ & $e^{-}$ & $\nu_{e}$ & $\overline{\nu_{\mu}}$ \\
\hline$L$ & +1 & $=$ & +1 & +1 & -1 \\
\hline$L_{e}$ & 0 & $\neq$ & +1 & +1 & 0 \\
\hline$L_{\mu}$ & +1 & $\neq$ & 0 & 0 & -1 \\
\hline
\end{tabular}

is presented in the gauge symmetry with the flavor quantum states of quarks and leptons. While the quarks and charged leptons obtain their masses under the mechanism that spontaneously breaks the gauge symmetry, the interaction is generated in the representation of the mass quantum states. The quark mixture of the flavor and mass quantum states is presented in the form of the CKM matrix, and only appears for the $\mathrm{CC}$ interaction, not for the $\mathrm{NC}$ interaction $[4,6,5]$. Unlike the quark sector, the quantum mixture has never been introduced in the lepton sector, due to massless neutrino. The SM-like neutrinos can only couple to their own charged leptons. For example, the electron type neutrino can only couple to electron. Thus, the mixing matrix is just unity in the lepton sector. However, if neutrino has mass, then the flavor state of $\left|\nu_{l}\right\rangle$ can be mixed with the mass quantum states of $\left|\nu_{\alpha}\right\rangle$. The electron neutrino can not only couple to electron, but also to muon and tau neutrinos. The 
same is true for the other neutrinos. The quantum mixture can be written in the form:

$$
\left|\nu_{l}>=\sum_{\alpha=1}^{3} U_{l \alpha}^{*}\right| \nu_{\alpha}>
$$

where $U_{l \alpha}$ is a unitary matrix and represents weak coupling strength of flavor of $l$ to physical states of $\alpha$. This analogy is applied to that in lepton sector from the CKM mixing matrix in quark sector. The matrix $U$ is so called PMNS (Pontecorvo-MakiNakagawa-Sakata) mixing matrix $[22,23,6]$. It is often written in the form:

$$
\begin{array}{r}
U_{P M N S}=\left(\begin{array}{ccc}
1 & 0 & 0 \\
0 & \cos \theta_{23} & \sin \theta_{23} \\
0 & -\sin \theta_{23} & \cos \theta_{23}
\end{array}\right)\left(\begin{array}{ccc}
\cos \theta_{13} & 0 & \sin \theta_{13} e^{-i \delta} \\
0 & 1 & 0 \\
-\sin \theta_{13} e^{i \delta} & 0 & \cos \theta_{13}
\end{array}\right) \\
\times\left(\begin{array}{ccc}
\cos \theta_{12} & \sin \theta_{12} & 0 \\
-\sin \theta_{12} & \cos \theta_{12} & 0 \\
0 & 0 & 1
\end{array}\right)\left(\begin{array}{ccc}
1 & 0 & 0 \\
0 & e^{i \phi_{1}} & 0 \\
0 & 0 & e^{i \phi_{2}}
\end{array}\right)
\end{array}
$$

where left, middle and right sub matrices are related to the mixture of neutrino 2-3, 1-3 and 1-2, which are related to atmospheric, reactor and solar neutrino sources. CP violating phase of $\delta$ is only apparent in the 1-3 sector. The last matrix is associated with the Majorana phases of $\phi_{1}$ and $\phi_{2}$. Measurements of those angles and the CP phase are described in next section. 


\section{B. Neutrino Mass Measurement}

Strong evidence of neutrino mass was confirmed first in the atmospheric neutrino oscillation sector. Currently the experimental study of neutrino mass is in the 2nd stage to fill out the mass ordering patterns of the three active neutrinos. There are three possible patterns for the mass ordering based on the observed mixing patterns. The first pattern is that of a pair of $\nu_{1}$ and $\nu_{2}$ is lighter than $\nu_{3}$, then the mass ordering is called normal mass hierarchy $\left(m_{1} \ll m_{2} \ll m_{3}\right)$. $\nu_{1}$ is the lightest neutrino mass in this scheme and the dominant component of $\nu_{e}$. This mass pattern is similar to the mass ordering of quarks and charged leptons. On the other hand if the pair is heavier than the $\nu_{3}$, then the order is called an inverted mass hierarchy $\left(m_{3} \ll m_{1} \approx m_{2}\right)$ and $\nu_{3}$ is the lightest neutrino mass. The last pattern is approximately degenerate if all masses are large compared to their differences $\left(m_{1} \approx m_{2} \approx m_{3}\right)$. A schematic of the normal and inverted mass hierarchies is shown in Figure 1.

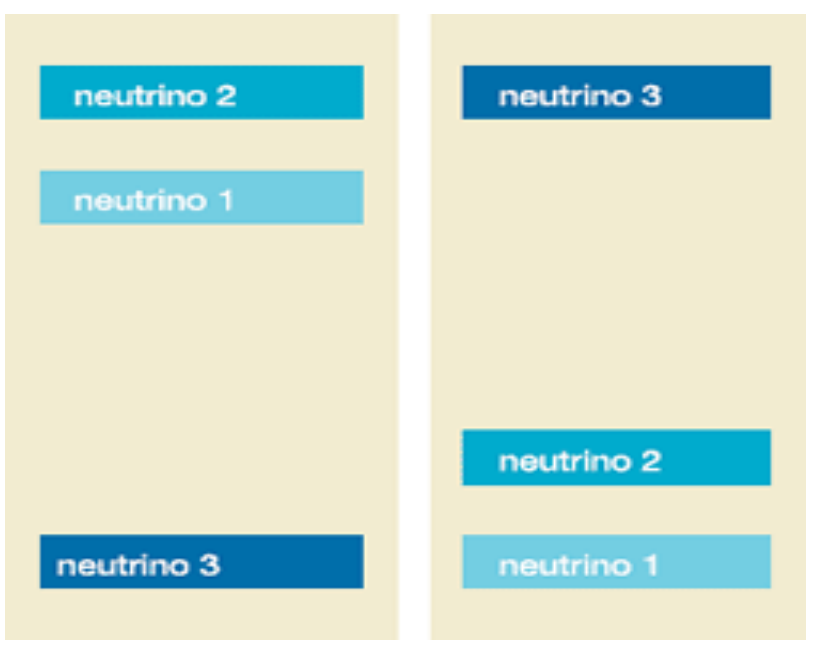

FIG. 1: Neutrino mass hierarchy. $\nu_{3}$ is the lightest neutrino in the inverted hierarchy. $\nu_{1}$ is the lightest neutrino in the normal hierarchy. 
Neutrino masses can be measured in two distinct ways. First, the experimental search for neutrinoless double beta decays is one of the possible ways to measure the "absolute" mass scale. The SM predicts the double beta decay and the process is experimentally confirmed by Elliot, Halin and Moe in 1987 [24]. However, if the neutrino is a Majorana particle, then the neutrinoless double beta decay is one of the allowed nuclear processes and violates the lepton number symmetry without being accompanied by $\mathrm{RH}$ neutrinos. The RH neutrino is absorbed at the second vertex as a LH neutrino since neutrino and anti-neutrino are identical. Only two electrons are emitted at the final states. The process is written in the form of $(Z, A) \rightarrow$ $(Z+2, A)+2 e^{-}$. It is one of the most important measurements in neutrino physics, but it is outside the scope of this dissertation work. Details are described in Ref. $[12,15,16,17,18,19,20,21]$. Also the other possible measurements are discussed in Ref. [25, 26, 27, 28, 29, 30, 31, 12, 32, 33].

The second way is to measure "relative" mass scale between two neutrinos. This can be done by searching for neutrino oscillations, which is the quantum phenomena that allows one flavor state to transform into another state as a neutrino travels through space. Assume that a particular type of flavor state propagates in time. If a neutrino has mass, then the flavor state $\mid \nu_{l}(0)>$ is converted with a superposition of mass state $\mid \nu_{\alpha}(0)>$. The unitary matrix is shown in Equation 2.2. The final state is calculated by applying a time evolution operator. The neutrino travels near the speed of light and time can be converted into the distance traveled and energy component given by $E_{\alpha}=\sqrt{p^{2}+m_{\alpha}^{2}} \approx p+\frac{m_{\alpha}^{2}}{2 p}$. Those steps are written as follows;

$$
\left|\nu_{l}(t)>=e^{-i H t}\right| \nu_{l}(0)>=\sum_{\alpha} U_{l \alpha} e^{-i E_{\alpha} t} \mid \nu_{\alpha}(0)>
$$




$$
\approx \sum_{\alpha} U_{l \alpha} e^{-i\left(p+\frac{m_{\alpha}^{2}}{2 p}\right) L} \mid \nu_{\alpha}(0)>
$$

Finally the probability of finding state $\nu_{l^{\prime}}$ in the $\nu_{l}$ beam is written in the form

$$
\begin{aligned}
P_{\nu_{l} \rightarrow \nu_{l^{\prime}}}(t) & =\left|<\nu_{l^{\prime}}(0)\right| \nu_{l}(t)>\left.\right|^{2} \\
& =\delta_{l l^{\prime}}-4 \sum_{\alpha>\beta}\left|U_{l \alpha} U_{l^{\prime} \alpha}^{*} U_{l \beta}^{*} U_{l^{\prime} \beta}\right| \sin ^{2}\left(1.27 \Delta \mathrm{m}_{\alpha \beta}^{2} \mathrm{~L} / \mathrm{E}\right)
\end{aligned}
$$

where $\Delta m_{\alpha \beta}^{2}, L$ and $E$ are mass difference squared of two the neutrinos, distance in $\mathrm{km}$ and initial neutrino energy in $\mathrm{GeV}$ respectively. The mass differences and mixing angles in the probability formula are related to the frequency of the oscillation and probability of detecting neutrino events. The phenomenology of neutrino oscillation can simplify depending on the $L / E$ ratio associated with a given experiment. Details are described in Ref. [12]. A review of the neutrino oscillation measurements door to date are discussed in the following sections. 


\section{Solar Neutrinos}

Neutrino oscillations associated with the 1-2 mixing sector can be measured using the neutrinos generated through the process of the nuclear fusion in the core of the sun. Those neutrinos are one of the important astrophysical sources of neutrinos which help us understand the internal structure and evolution of the sun and other stars. Only $\nu_{e}$ 's are generated in those reactions with energies roughly in the range of a few $\mathrm{MeV}$, depending on details of nuclear reactions. A neutrino's mean free path is of the order of $10^{17} \mathrm{~cm}$ hence large enough that they can get out of the sun and be detected on Earth using underground detectors. If the neutrino has mass, the solar neutrino flux (or detection rate) varies with a $\nu_{e}$ survival probability depending on $\theta_{12}$ and $\Delta m_{21}^{2}$, and the $\mathrm{L} / \mathrm{E}$ input, where $L$ is the distance between the sun and Earth and $E$ is the solar neutrino energy.

Table II: Solar neutrino flux. Solar neutrino flux obtained from various experiments. SNO experiment is sensitive to measure the flux for $\mathrm{ES}, \mathrm{CC}$ and $\mathrm{NC}$ interaction channels. The other experiments are only sensitive to the single interaction channel as indicated. The flux ratio means the ratio of the observed to expected SSM flux.

\begin{tabular}{|c|c|c|}
\hline Experiment & Type & Flux Ratio \\
\hline \hline Homestake & CC & $0.27 \pm 0.03$ \\
\hline Kamiokande & ES & $0.44 \pm 0.06$ \\
\hline SAGE & CC & $0.553 \pm 0.034$ \\
\hline Gallex & CC & $0.579 \pm 0.037$ \\
\hline \hline \multirow{3}{*}{ SNO } & CC & $0.349 \pm 0.021$ \\
\cline { 2 - 3 } & ES & $0.473 \pm 0.052$ \\
\cline { 2 - 3 } & NC & $1.008 \pm 0.123$ \\
\hline
\end{tabular}


In last past 60 years the solar neutrino flux has been measured in various experiments $[34,35,36,12]$. All experimental results are consistent with the solar neutrino detection rate being much lower than the theoretical expected value quoted by the Standard Solar Model(SSM) $[37,38,12]$. Table II and Figure 2 show that the ratio of the observed to the expected SSM flux and the flux as a function of the neutrino energy for each nuclear reaction. The current best measurement of the solar neutrino flux is made by the SNO experiment located in INCO Ltd's Creighton Mine near Sudbury, Canada $[39,40,12]$. This experiment uses a Cerenkov detector filled with heavy water, that is sensitive to measure the flux of ${ }^{8} B$ neutrinos ${ }^{2}$ for three different interaction channels; Elastic Scattering(ES), Charged and Neutral Current interactions (CC and NC). (1) CC channel only occurs for $\nu_{e}$ and has an advantage that outgoing electron's energy is strongly correlated to incident neutrino energy. ES interaction is sensitive to all neutrino flavors, making the $\nu_{e}$ cross section is approximately 1.5 times larger than $\nu_{\mu}$ and $\nu_{\tau}$. The results from those two channels is that flux obtained from CC interaction is slightly lower than that obtained by ES interaction. The difference between CC and ES results indicates that the flux is obtained by $\nu_{\mu}$ and $\nu_{\tau}$ components. (2) NC channel is equally sensitive to all neutrino flavors and possible to measure the total flux of ${ }^{8} B$ neutrinos. The result is that the flux obtained from NC interaction is completely consistent with the prediction of the SSM calculation. Thus the phenomenology of the solar neutrinos is well explained by the $\nu_{e}$ disappearance

\footnotetext{
${ }^{2}$ Relatively high energy neutrinos are detected with the SNO detector for the purpose of enhancing the matter effects that neutrinos experience from the production region until they exit the sun. As neutrinos propagate in matter, the additional contribution of $+\sqrt{2} G_{F} N_{e}$ appears in Hamiltonian of $\overline{\nu_{e}}+e$ scattering, where $G_{F}$ is the Fermi constant and $N_{e}$ is the number density of electrons in matter. This matter effect is proportional to the neutrino energy, and can enhance or reduce the true values of mass eigenstates and mixing angles. The flux of ${ }^{8} B$ neutrinos is dominant at relatively high energy and largely affected by the matter and undergoes matter oscillations. The survival probability must be altered to account for these matteroscillations [12].
} 
via matter-oscillation hypothesis. More details are described in Ref. [12].

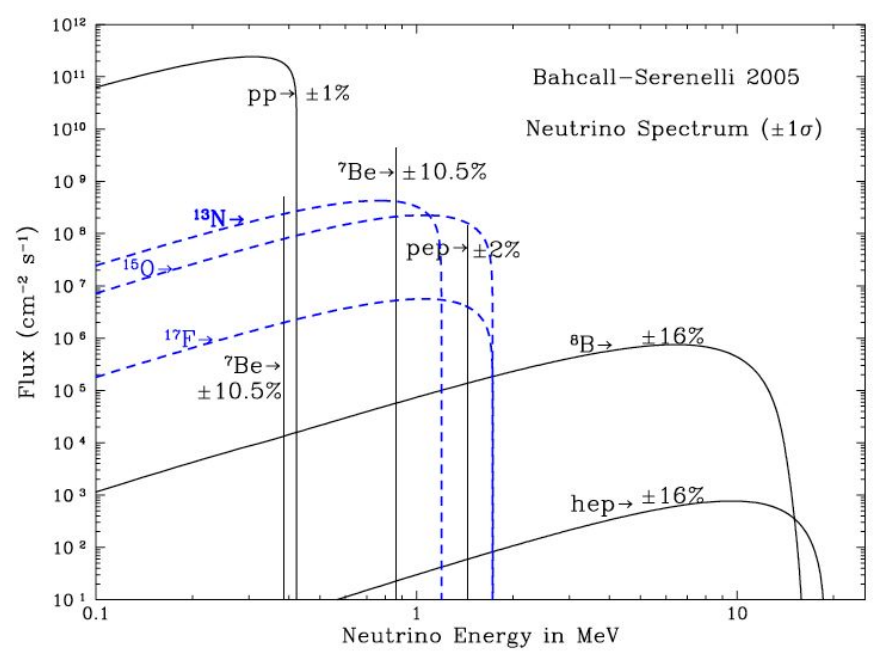

FIG. 2: Solar neutrino flux. The SSM flux is shown as a function of neutrino energy for different nuclear fusion channels [38].

The KamLAND experiment was staged to provide a test of the solar neutrino oscillation and one of the precision experiments being used to carry out measurements of $\theta_{12}$ and $\Delta m_{12}^{2}[41,12]$. Their experiment is located at the Kamioka mine in Japan and is designed to search for $\overline{\nu_{e}}$-disappearance via neutrinos produced by 55 Japanese nuclear power reactors. The experiment is impacted by two input parameters; (1) the distance that neutrino travels through space and (2) energy range of neutrino detection. The average distance between the KamLAND detector and the reactors is about $180 \mathrm{~km}$. Secondary, $\overline{\nu_{e}}$ detections are made via the inverse beta decay reaction; $\overline{\nu_{e}}+p \rightarrow e^{+}+n$ with a threshold $1.8 \mathrm{MeV}$. Neglecting the small neutron recoil, the anti-neutrino energy is constructed by the sum of the positron and $0.8 \mathrm{MeV}$ of $e^{+} e^{-}$ annihilation energy and measured with a resolution of $6.2 \% / \sqrt{E(M e V)}$. The typical energy range is from 1.8 to $10 \mathrm{MeV}$. Thus if CPT is conserved and matter-enhanced 
neutrino oscillation (MSW effect) is the underlying mechanism for the observed flavor transformation in the solar neutrinos, then the experiment would expect a significant fraction of $\overline{\nu_{e}}$ oscillation at the $L / E$ relation of 1.8 to $180 \mathrm{~km} / \mathrm{MeV}$. The results of a $2 \mathrm{D}$ oscillation $\mathrm{fit}^{3}$ is shown in Figure 3. Their data is consistent with the solar neutrino result with the best fit of $7.58_{-0.13}^{+0.14}$ (stat.) \pm 0.15 (syst.) $\times 10^{-5} \mathrm{eV}^{2}$ and mixing angle $0.56_{-0.07}^{+0.10}(\text { stat. })_{-0.06}^{+0.10}($ syst.) at $68 \%$ confidence level.
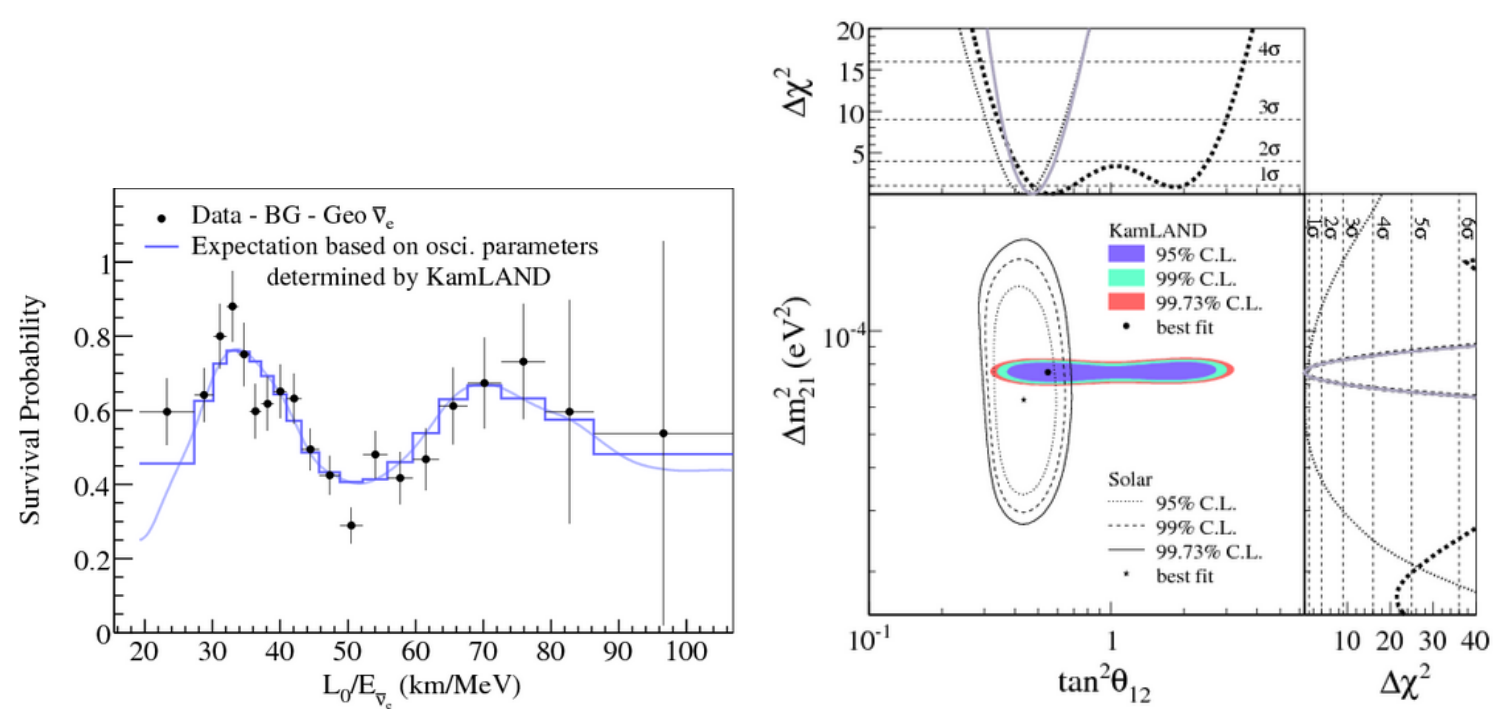

FIG. 3: Result from KamLAND experiment. (Left) $L / E$ spectrum shows that neutrino is maximally oscillated around $L / E \sim 50 \mathrm{~m} / \mathrm{MeV}$. (Right) Black line shows the result from solar neutrino data. The color contour shows the fitting result from KamLAND data for the total exposure of $2.44 \times 10^{32}$ proton-yr (2881 ton-yr). These figures are referenced from [41].

${ }^{3}$ The $2 \mathrm{D}$ and $1 \mathrm{D}$ oscillation fits are defined in this dissertation. The $\Delta m^{2}$ and $\sin ^{2}(2 \theta)$ oscillation parameters are treated as free parameters in the $2 \mathrm{D}$ oscillation fit. On the other hand, the 1D oscillation fit only treats the $\Delta m^{2}$ parameter as a free parameter while the $\sin ^{2}(2 \theta)$ parameter is constrained to maximal value. See the section IV.C.3. 


\section{Atmospheric Neutrinos}

Neutrino oscillations associated to the 2-3 mixing sector can be measured using the neutrinos produced in the atmosphere by cosmic ray collisions. In 1998, precision measurements of atmospheric neutrino oscillations were made by the Super Kamiokande (SK) detector in Japan for the first time $[42,43,12]$. Their analysis was performed by calculating the $\nu_{\mu}$ disappearance probability using the directional distributions of muon neutrinos produced in the atmosphere. The probability can be written as

$$
\mathrm{P}_{\nu_{\mu} \rightarrow \nu_{\mu}} \approx 1-\sin ^{2}\left(2 \theta_{23}\right) \sin ^{2}\left(1.27 \Delta \mathrm{m}_{32}^{2} \mathrm{~L} / \mathrm{E}\right)
$$

which is derived using Equation (2.4) and taking a limit of $\theta_{23} \gg \theta_{13} \sim 0$ and $\Delta m_{32}^{2} \approx \Delta m_{31}^{2} \gg \Delta m_{21}^{2}$. If a neutrino has mass and mixing, then the oscillation probability changes depending on $L / E$ input. $L$ is the distance between neutrino production point in the atmosphere and detection point where the SK detector is located. The distance depends on the direction that the neutrino travels in the detector. $E$ is the neutrino energy reconstructed by collecting the Cerenkov light produced by the interaction products in water. Their result is shown in Figure 4 . The ratio of the data to the Monte Carlo (MC) simulation events is shown as a function of the reconstructed $L / E$ with the best-fit expectation for muon neutrino oscillation (solid line). Clearly the oscillation is maximal at $L / E \sim 500 \mathrm{~km} / \mathrm{GeV}$.

The NuMI-MINOS experiment was planned to provide a test of the atmospheric neutrino oscillation hypothesis and designed to make a high statistics measurement of the $\nu_{\mu}$ disappearance using a neutrino beam $[9,44,45]$. Their latest analysis was 

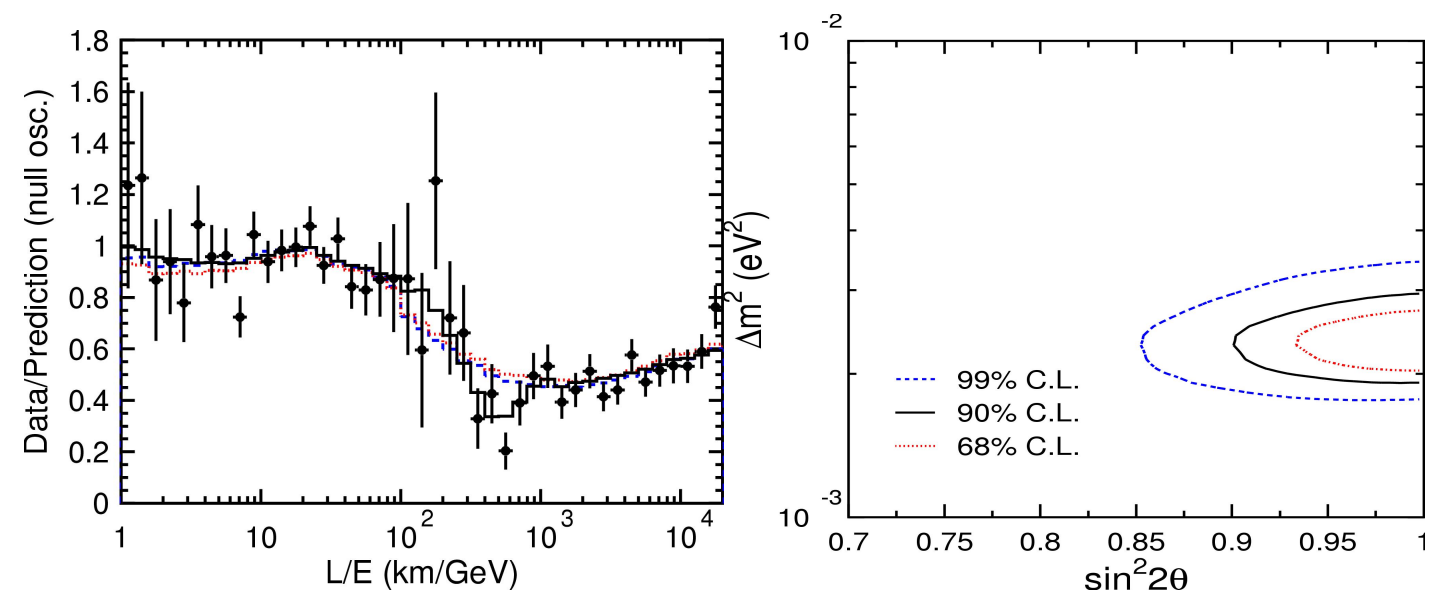

FIG. 4: SK result of atmospheric neutrino. (Left) SK result of atmospheric neutrino $L / E$ spectrum. The best-fit expectations are shown for neutrino oscillation (solid line) and decay (dashed line) and decoherence (dotted line). (Right) 68\%, 90\% and 99\% C.L. allowed oscillation parameter regions. These figures are referenced from $[42]$.

performed with CC events from a total data set produced by $3.36 \times 10^{20}$ protons on target (POT) from May 2005 to July 2007. For the low energy beam configuration, 730 events are observed with the expectation of $936 \pm 53$ (syst) events for the energy range of 1-5 GeV. The observed and expected energy spectrum and their ratio are shown in Figure 5. The data is consistent with muon neutrino oscillation with a mass difference $\left|\Delta m_{32}^{2}\right|=2.43 \pm 0.13 \times 10^{-3} \mathrm{eV}(68 \%$ confidence level $)$ and a mixing angle $\sin ^{2}\left(2 \theta_{23}\right)>0.90$ (90\% confidence level). The best fit $\chi^{2}$ is 90 for 97 degree of freedom. The result of the $2 \mathrm{D}$ oscillation parameter fitting is shown in Figure 6 . In addition, if the fit is not constrained to the physical region, then the result is $\left|\Delta m_{32}^{2}\right|=2.33 \times 10^{-3} \mathrm{eV}^{2}$ and $\sin ^{2} 2 \theta=1.07$, with a 0.6 unit decrease in $\chi^{2}$. The largest systematic effects were found to be (1) a $4 \%$ uncertainty of the predicted 

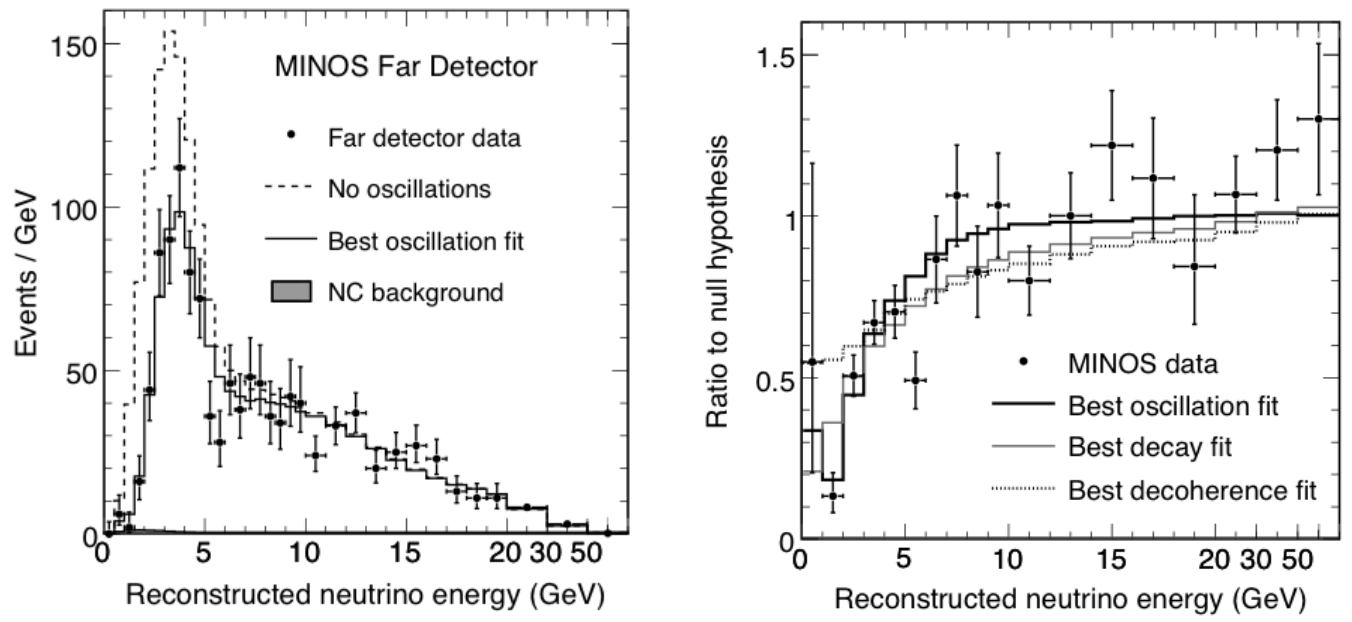

FIG. 5: Neutrino energy distribution and ratio from MINOS experiment. Expected and observed CC energy spectrum at the Far Detector is shown at left. The oscillated depletion is shown around $3 \mathrm{GeV}$. The ratio of data to $\mathrm{MC}$ is shown at right. These figures are referenced from [9].

Far Detector event rate, which is the sum in quadrature of the uncertainties on the detector's fiducial mass, event selection efficiency and the POT counting accuracy, (2) a $10 \%$ uncertainty of the absolute hadronic energy scale and (3) $50 \%$ uncertainty of neutral current background in the CC event samples.

Sensitivity of the MINOS oscillation measurement is evaluated by performing a unconstrained fit to high statistics "fake" (or mock) data sets weighted to the central values of $\Delta m^{2}=2.45 \times 10^{-3} \mathrm{eV}^{2}$ and maximal mixing [46]. The $68 \%$ and $90 \%$ C.L. $\Delta m^{2}$ and mixing sensitivities are shown as a function of the POT exposure for the low energy beam configuration in Figure 7. The $\Delta m^{2}$ and mixing allowed regions from the MINOS data set of $3.36 \times 10^{20}$ POT are shown in blue bar and red point. These data are making the allowed regions smaller than the sensitivities from the 


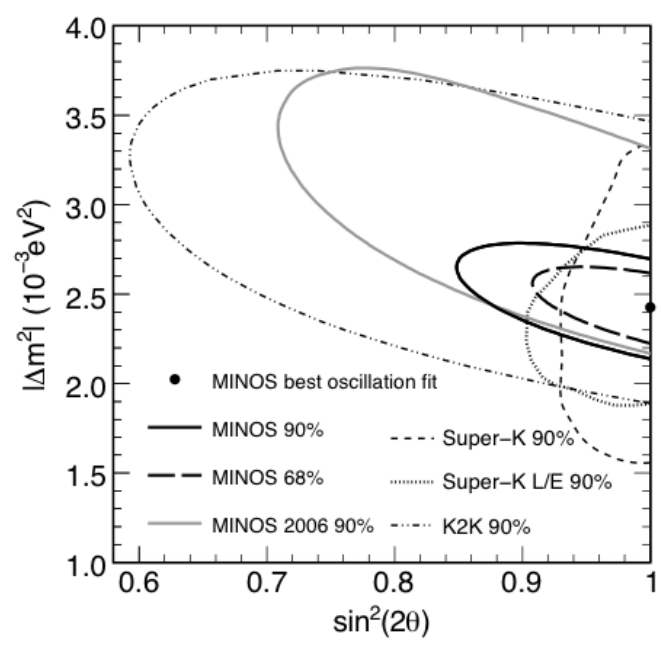

FIG. 6: Oscillation contour from MINOS experiment. The oscillation contour of $\Delta m^{2}$ vs $\sin ^{2}(2 \theta)$ is shown in the figure. This figure is referenced from [9].

"fake" (or mock) data set since the best fit point is located at the unphysical region $\left(\sin ^{2}(2 \theta)=1.07\right)$.

\section{3. $\nu_{e}$ Appearance}

A challenge for next generation experiments is to observe $\nu_{\mu} \rightarrow \nu_{e}$ oscillation in the atmospheric oscillation sector. The main targets are to measure the values of $\theta_{13}$, the sign of $\Delta m_{32}^{2}$ and the $\mathrm{CP}$ violating phase. The measurement of a non-zero value of $\theta_{13}$ is a critical step to measure the CP phase, which may hold the answer to the questions of matter-antimatter symmetry of the lepton sector in our universe. Currently the $\mathrm{T} 2 \mathrm{~K}$ and $\mathrm{NO} \nu \mathrm{A}$ experiments are under development and designed to search for this oscillation mode using an off-axis neutrino beam [47, 48, 49, 50, 48]. Primary protons hit a target to create neutrino beam. High energy collision with the target produces many secondary hadrons, mostly pions and kaons. Neutrinos are 

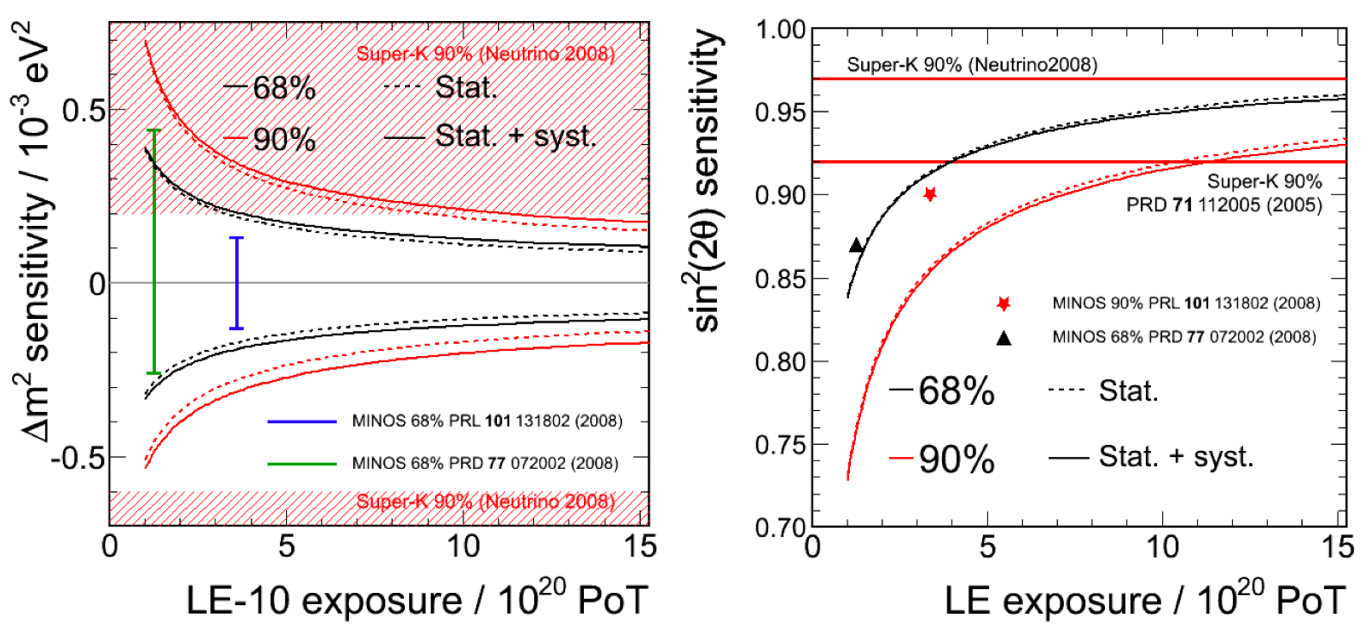

FIG. 7: Limitation of MINOS experiment. Left and right figures show the sensitivity for $\Delta m^{2}$ and the mixing angle measurements as a function of the POT exposure for the low energy beam configuration. $68 \%$ and $90 \%$ confidence level regions are for black and red line. The systematic error is added for the dashed line. Red straight and shaded region are for SK 90\% confidence limit. These figures are referenced from $[46]$.

produced at small angles from the pion decay in flight. The neutrino energy is related to the pion energy through the following formula, $E_{\nu}=\frac{0.43 E_{\pi}}{1+\gamma^{2} \theta^{2}}$ where $\gamma=E_{\pi} / m_{\pi}$, $E_{\pi}$ and $m_{\pi}$ are pion energy and mass. While the neutrino energy is proportional to pion energy on-axis, the neutrino energy is less dependent on pion energy and largely depends on off-axis angle. The basic configuration for the $\mathrm{T} 2 \mathrm{~K}$ and $\mathrm{NO} \nu \mathrm{A}$ experiments is the same as most long baseline neutrino oscillation experiment such as the K2K and MINOS [51,9]. T2K experiment uses an off-axis neutrino beam created at the J-PARC accelerator facility built in Tokai, Japan, which is pointed 2-3 degrees off-axis to the SK detector located $295 \mathrm{~km}$ away from the target position. 

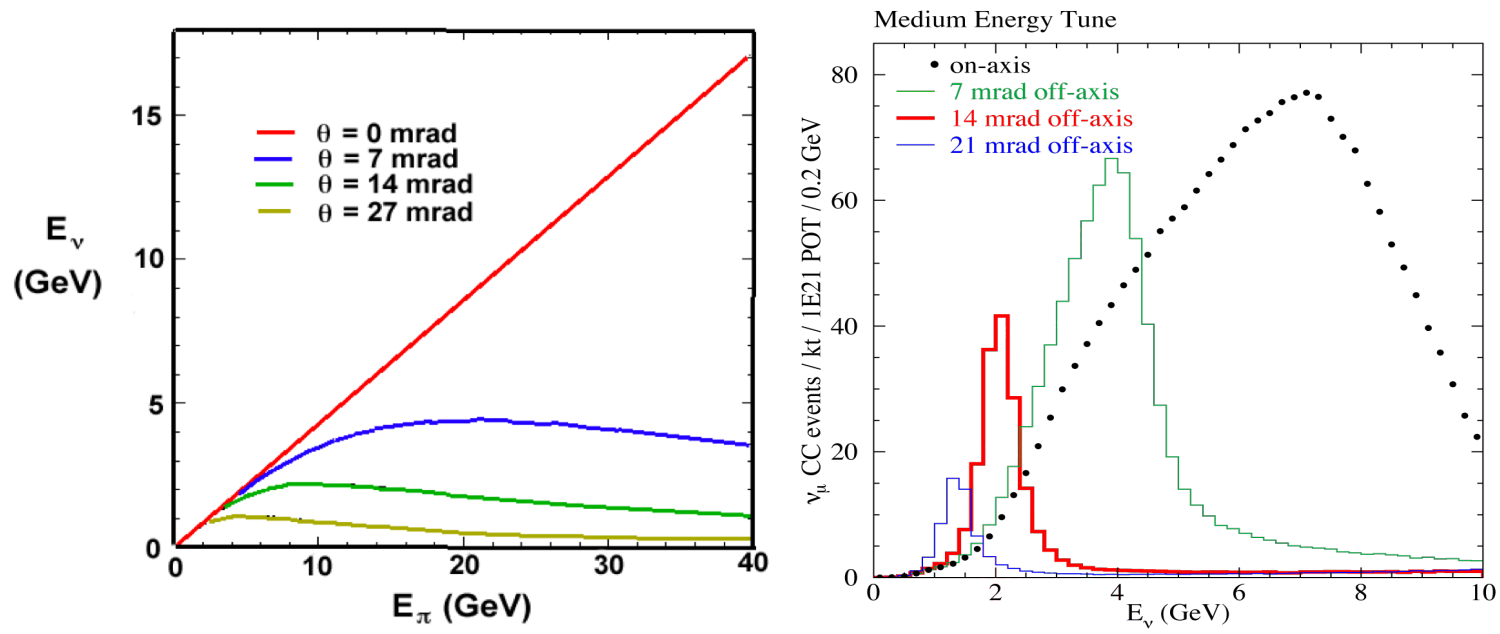

FIG. 8: NuMI off-axis beam spectrum. (Left) Red line shows that the the relativistic relation between pion and neutrino on the beam axis. The neutrino is less dependent to pion energy for small angle off the beam axis. (Right) Black points show the neutrino energy spectrum for the NuMI medium beam energy configuration on-axis. The spectrum is shifted near the maximally oscillated position if the off-axis angle is $14 \mathrm{mrad}$ and shown in red histogram. These figures are referenced from [49].

The neutrino energy spectrum is configured to peak at $\sim 600 \mathrm{MeV}$ and maximally oscillated at $\sim 491 \mathrm{~km} / \mathrm{GeV}$. Similarly, NO $\nu$ A's Far detector is planned to be built $810 \mathrm{~km}$ away from the target position at the Ash River Trail in MN and off the center of the NuMI neutrino beamline. Figure 8 left shows the kinematic relation between neutrino energy and off-axis angle. Figure 8 right shows that the energy of 14.5 mrad off-axis ME beam configuration is peaked at $2 \mathrm{GeV}$. Thus $\mathrm{NO} \nu \mathrm{A}$ 's neutrino energy spectrum is configured to peak at $\sim 2 \mathrm{GeV}$ and maximally oscillated at $\sim 405$ $\mathrm{km} / \mathrm{GeV}$. 
The $\mathrm{T} 2 \mathrm{~K}$ and $\mathrm{NO} \nu \mathrm{A}$ experiments have a capability to access the 1-3 sector in the PMNS matrix and to determine the mass hierarchy shown in Figure 1. If $\Delta m_{13}^{2}>0$, then $\nu_{3}$ is heavier than $\nu_{1}$ and the mass ordering is the normal mass hierarchy. On the other hand, if $\Delta m_{13}^{2}<0$, then the hierarchy is inverted. Assuming that the experiments run for 3 years each of neutrino and antineutrino modes with the 700 $\mathrm{kW}$ beam power, the sensitivity to measure $\theta_{13}$ is shown as a function of $\mathrm{CP}$ violating phase $\delta$ and mass hierarchy in Figure 9. The three standard deviation sensitivities are approximately an order of magnitude beyond the $90 \%$ confidence level set by CHOOZ experiment of $\sin ^{2} \theta_{13}<0.13$ at $\left|\Delta m_{32}^{2}\right|=2.7 \times 10^{-3} \mathrm{eV}^{2}$. Also the mass hierarchy is strongly correlated to the apparent $\mathrm{CP}$ violating phase caused by the matter effects in the earth. The sensitivity to measure the mass ordering is shown as a function of the CP phase in Figure 10, but the measurement is only possible if the $\sin ^{2} \theta_{13}$ is near the CHOOZ limit. In addition, Double Chooz experiment is currently under construction and sensitive to measure those three parameters. Unlike the previous two experiments, it is sensitive to search for $\overline{\nu_{\mu}}$ appearance via anti-electron neutrinos produced from the reactor cores at the CHOOZ nuclear power plant in the Ardennes region of northern France [52, 48]. 


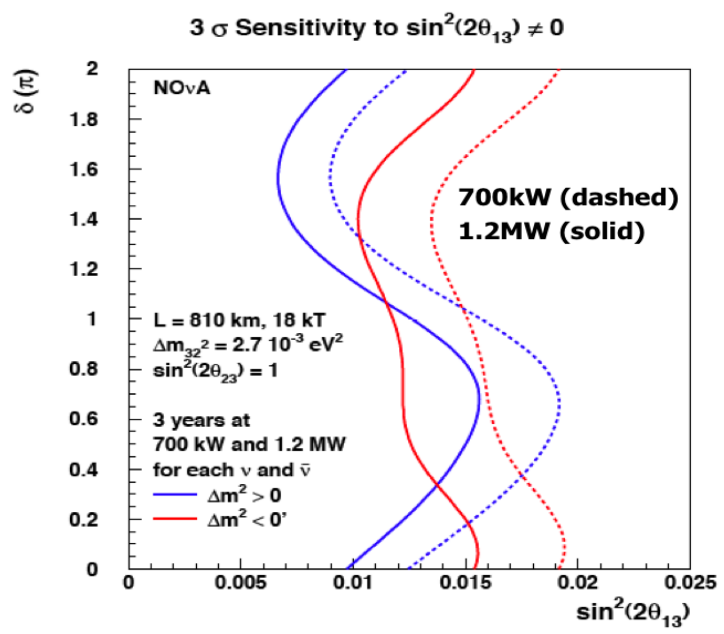

FIG. 9: $\theta_{13}$ sensitivity contour in the $\mathrm{NO} \nu \mathrm{A}$ experiment. $\sin ^{2}\left(2 \theta_{13}\right)$ sensitivity contour for $700 \mathrm{~kW}$ beam power are shown in the top figure as a function of the CP phase in solid line. The figure is referenced from [50].
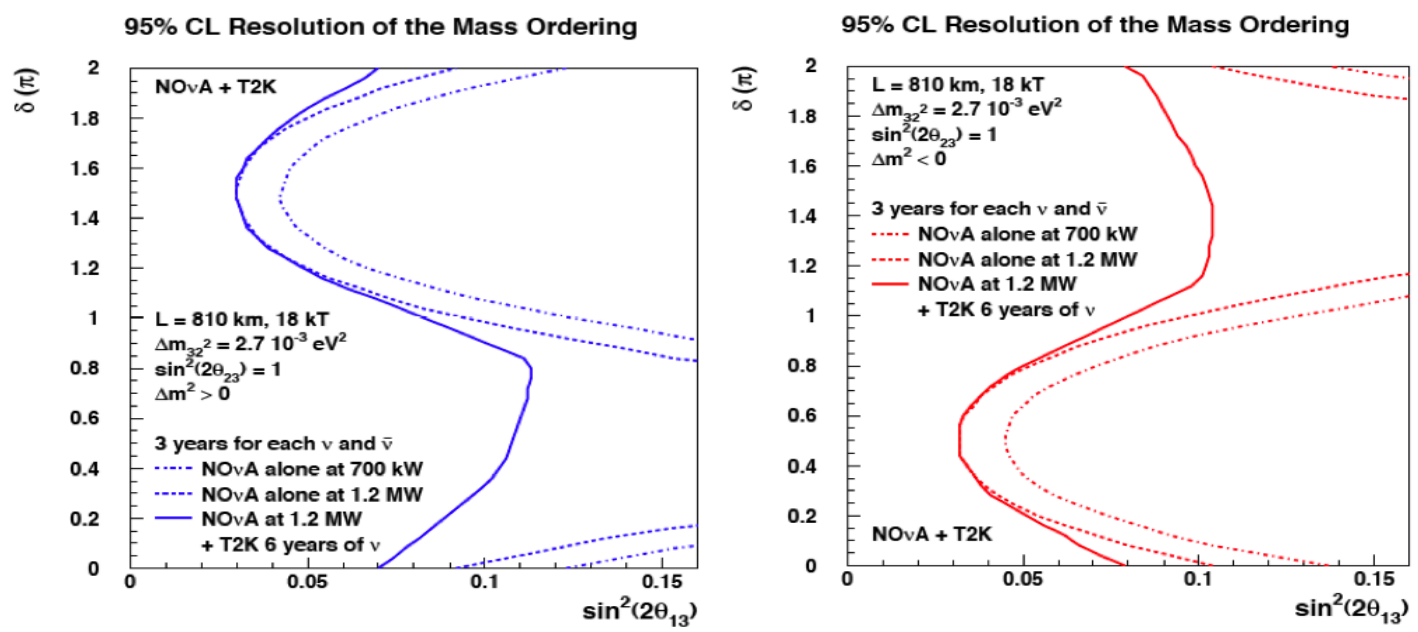

FIG. 10: Mass ordering sensitivity in the $\mathrm{NO} \nu \mathrm{A}$ experiment. Normal and inverted mass hierarchy are in red and blue color. Mass ordering sensitivity contours for 700 $\mathrm{kW}$ beam power are shown as a function of the CP phase in dash-dot line. The left and right bottom figures are for normal and inverted hierarchy. These figures are referenced from [50]. 


\section{Light Neutrino Mass Generation Mechanism}

Neutrinos appear to be very light compared to the other fundamental particles. GUTs should be able to explain the mechanism to generate a heavy top quark and light neutrinos. It is called mass hierarchy problem. The mass spectrum for quarks and leptons is shown in Figure 11. Only upper limits are shown for neutrino mass. If one takes the ratio of the top quark to electron mass and electron to electron neutrino mass, then a simple calculation shows two things; (1) the top quark is roughly five orders of magnitude heavier than electron mass $\left(m_{t} / m_{e} \approx 3.38 \times 10^{5}\right)$ and (2) the electron neutrino is at least five orders of magnitude lighter than the electron $\left(m_{e} / m_{\nu_{e}} \gtrsim 5.11 \times 10^{5}\right)$. The question of light neutrino mass also remains even within a single family as well. For example, in the first family the electron neutrino is at least 5 orders of magnitude lighter than the up and down quarks and electron mass. Similarly, in the second family the muon neutrino is at least 3 orders of magnitude lighter than the charm and strange quarks and muon mass.

\section{Seesaw Mechanism}

There are many possible models to explain the mass mechanism. The SM can be simply extended by adjusting the context of Fermion and/or Higgs sector in the $S U(2)_{L} \times U(1)_{Y}$ group [12]. As an example model, the seesaw mechanism engaged with Fermion sector is introduced in this section. Charged leptons obtain their mass through the Higgs mechanism that spontaneously breaks the electroweak symmetry. Neutrinos also get their mass from the same mechanism. RH neutrino fields are assumed to be a neutral singlet and have no interaction with gauge bosons. Dirac and Majorana mass terms arise in the Lagrangian. The Dirac mass term can then be 


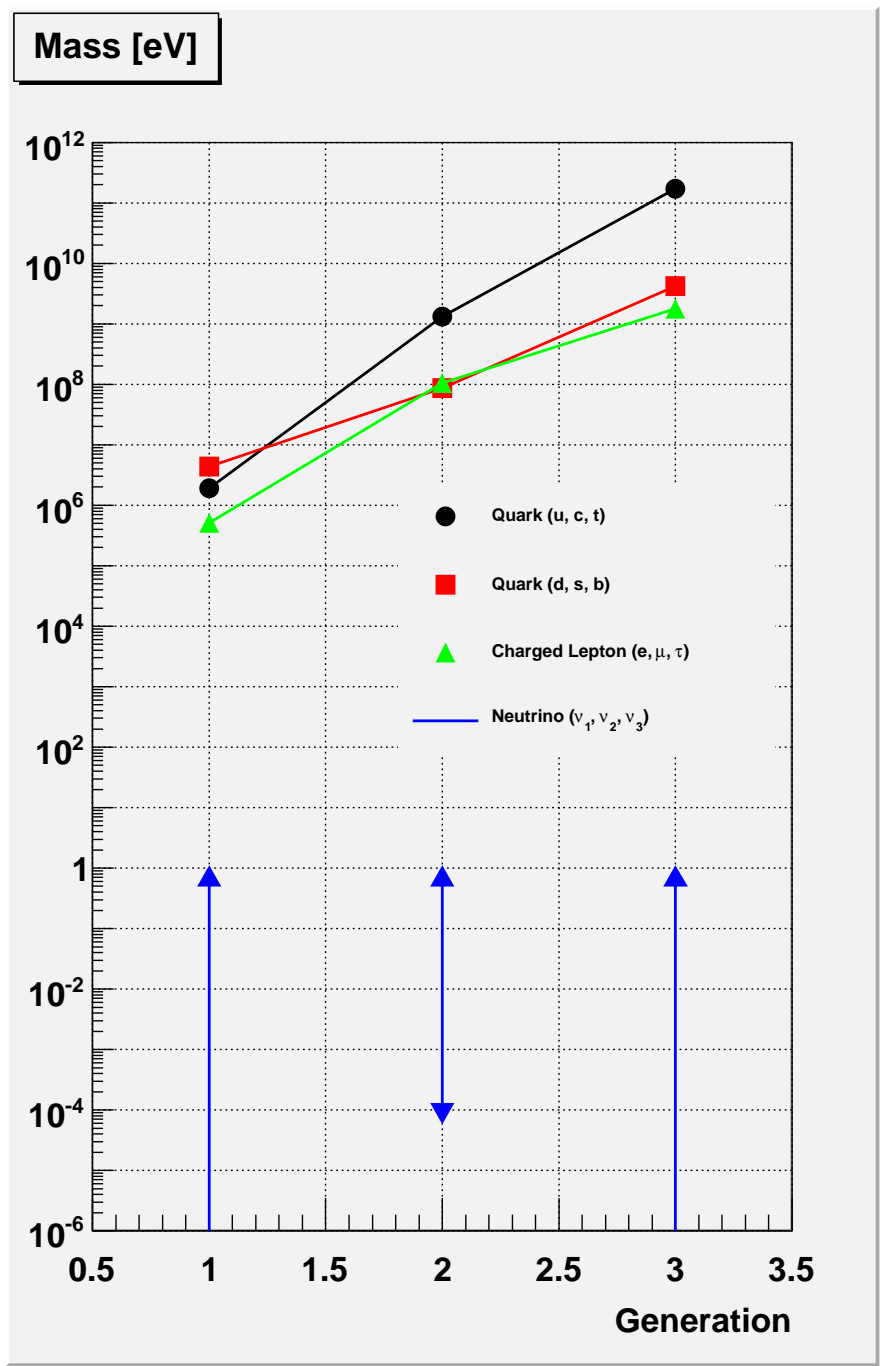

FIG. 11: Mass heirarchy. Vertical and horizontal axes show the mass scale in $\mathrm{eV}$ and 3 generations respectively. u-type and d-type quarks and charged leptons are marked in black, red and green points. Neutrinos and top quark are extremely light and heavy comparing to the other fundamental particles. Ranges for neutrino mass is shown in blue lines. 
written in the following form

$$
-m_{D}\left(\widehat{\nu_{L}} \nu_{R}+\nu_{L} \widehat{\nu_{R}}\right)
$$

Similarly, the Majorana mass term is in the form

$$
-\frac{1}{2} m_{L}\left(\widehat{\nu_{L} \nu_{L}}+\overline{\widehat{\nu_{L}}} \nu_{L}\right)-\frac{1}{2} m_{R}\left(\widehat{\nu_{R}} \overline{\nu_{R}}+\overline{\widehat{\nu_{R}}} \nu_{R}\right)
$$

Thus the Lagrangian can be written in the following form

$$
-L_{\text {mass }}=\frac{1}{2}\left(\begin{array}{cc}
\widehat{\nu_{L}} & \overline{\nu_{R}}
\end{array}\right)\left(\begin{array}{cc}
m_{L} & m_{D} \\
m_{D}^{T} & m_{R}
\end{array}\right)\left(\begin{array}{c}
\overline{\nu_{L}} \\
\nu_{R}
\end{array}\right)+h . c
$$

where $\nu$ is neutrino field and $\overline{\widehat{\nu}} \equiv \widehat{\nu}^{\dagger} \gamma_{0}=\nu^{T} C^{\dagger} \gamma_{0}^{\dagger} \gamma_{0}=\nu^{T} C^{-1}$ (C is the chargeconjugation operator). Eigenvalues of the mass matrix can be calculated and written in the form

$$
m_{1,2}=\frac{1}{2}\left[\left(m_{R}+m_{L}\right) \pm \sqrt{\left(m_{L}-m_{R}\right)^{2}+4 m_{D}^{2}}\right] \approx\left|m_{L}-\frac{m_{D}^{2}}{m_{R}}\right|, m_{R}
$$

where $m_{R} \gg m_{D} \gg m_{L} \sim 0$. If Dirac and heavy mass scales are $m_{D} \sim m_{\tau}$ and $m_{R} \sim M_{G U T}$, then a light neutrino mass can be naturally generated at eV scale. The lower bound for the Majorana mass is $m_{R} \gtrsim 5 \times 10^{9} \mathrm{GeV}$.

\section{Exotic Neutrino Search}

Most experimental results are consistent with three neutrino hypothesis that there are only three neutrino species; electron, muon and tau neutrinos. One neutrino is linked to only one family. These neutrinos weakly couple to the $\mathrm{Z}$ and $\mathrm{W}$ bosons. The hypothesis is confirmed by the measurements of the $\mathrm{Z}$ boson production width in LEP experiment [12]. Their result is $N_{\nu}=2.984 \pm 0.008$ (68\% C.L). Also the effective number of relativistic neutrino species can be calculated with cosmological 
data as well. The result from WMAP and the other cosmological experiments is $N_{\nu}=4.4 \pm 1.5(68 \%$ C.L), which is consistent with the standard value of 3.04 for the WMAP measurement [33]. However, it is possible to build theoretical models with extra species of neutrino.

(1) Heavy Majorana Neutrino : One way is to introduce new neutrinos associated to the conventional theory of weak interaction. For example, the seesaw mechanism is the one of the simple and reasonable models to explain light neutrino mass. Heavy RH neutrinos can be predicted around GUT scale to extract light LH neutrinos. If the Dirac mass scale is near the $\tau$ mass, then the heavy neutrino mass is predicted above $5 \times 10^{9} \mathrm{GeV}$. Details are described in section 2.C.1. Also supersymmetry theory opens up more questions and possibilities for new type of neutrinos. They are discussed in Ref. [53, 12, 54]. For current experimental technologies, it is impossible directly to access physics around the GUT scale.

(2) Light Sterile Neutrino : In 1995, the LSND collaboration reported their observation of $\overline{\nu_{e}}$ appearance in $\overline{\nu_{\mu}}$ beam at $\Delta m^{2} \sim 1 e V^{2}[55,56,57,12]$. This signal is maximally oscillated around $L / E \sim 0.7 \mathrm{~km} / \mathrm{GeV}$. This experimental result is not consistent with any other experimental results from solar and atmospheric neutrino oscillations. To explain this anomaly, one of the popular solutions is to introduce one or more extra neutrinos without any theoretical frameworks of weak interactions. These neutrinos are so called sterile neutrinos. Recently the MiniBooNE(MB) experiment performed a precision measurement in the region of the LSND signal at Fermilab, IL [58]. MB is configured with the fixed distance of $L=540 \mathrm{~m}$ and mean neutrino energy to be around $700 \mathrm{MeV}$, for the purpose of maximizing the LSND signal. In conclusion, their data is consistent with no oscillation and rules out the 
sterile neutrino hypothesis. Figure 12 shows null oscillation above $475 \mathrm{MeV}$. Details are described in Ref. $[12,59,60,61,62,63,64,65,66,67,68,69]$. Currently MB is running with anti-neutrinos, for the purpose of testing other possible exotic explanations such as the sterile neutrino hypothesis involving $\mathrm{CP}$ or even $\mathrm{CPT}$ violation. Their most recent result shows that their data agrees with MC background predictions as a function of neutrino energy and is consistent with no oscillation [70]. They will continue to take more data and to improve sensitivity to oscillation analysis.
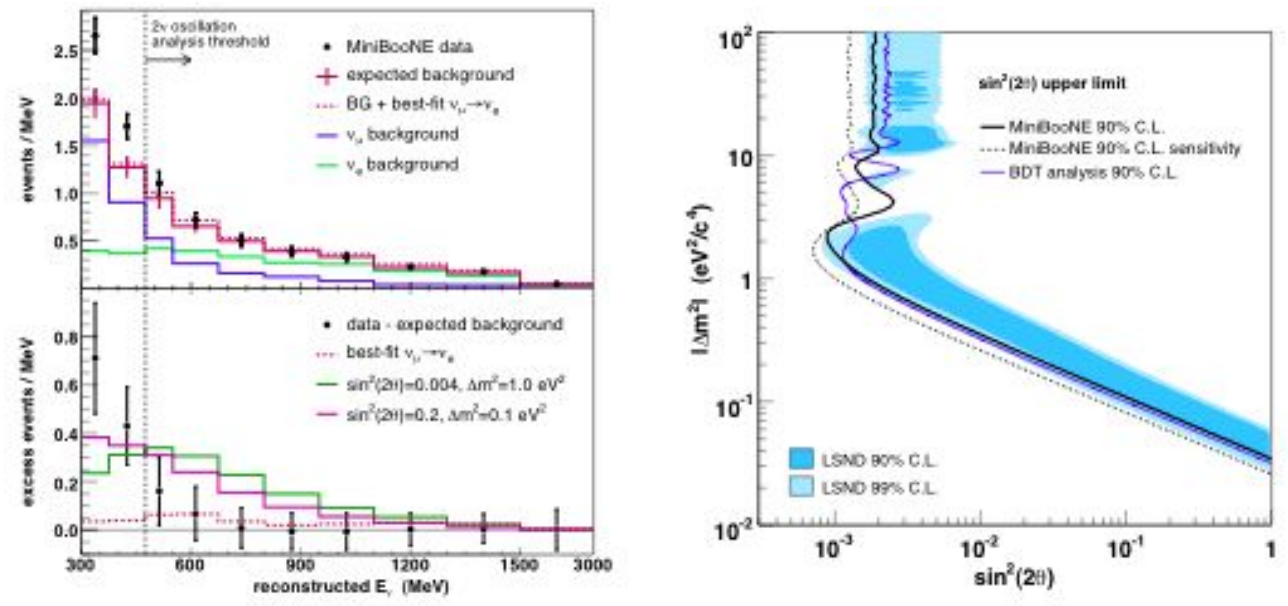

FIG. 12: Result from MiniBooNE experiment. (Left) Top figure shows the neutrino energy spectrum in MB. Bottom one is the ratio figure of the expected to observed spectra. MB data above $475 \mathrm{MeV}$ is consistent with no oscillation. (Right) $90 \% \mathrm{MB}$ exclusion region is shown with dashed and solid lines. LSND signal filled in blue is clearly excluded. Those figures are referenced from [58]. 


\section{CHAPTER III}

\section{THE NUMI-MINOS EXPERIMENT}

MINOS (Main Injector Neutrino Oscillation Search) experiment is especially designed to measure the neutrino mass differences and mixing angles in the atmospheric neutrino sector. The experiment is composed of 3 different components; the NuMI Neutrino Beamline, a Near Detector and a Far Detector. The beamline is created at Fermilab and pointed to both Near and Far detectors located at Fermilab and Soudan, MN respectively. The Near Detector is set only $1.0 \mathrm{~km}$ away from the target position, for the purpose of studying systematic variance and covariance of the beamline and extrapolating the unoscillated neutrino spectrums. The role of Far Detector is to project out the oscillated spectrum after traveling $735 \mathrm{~km}$. Each detector is designed in the same way so as to reduce the systematic effects of the neutrino flux, cross-section and detector acceptance. Each detector is capable of observing $\nu_{\mu}$ and $\nu_{e}$ charged and neutral current interactions having energy larger than $500 \mathrm{MeV}$. A toroidal magnetic field is installed to discriminate $\nu_{\mu}$ and $\overline{\nu_{\mu}}$ events. Details of the detectors are described in Ref. [44].

\section{A. NuMI Neutrino Beamline}

The Main Injector accepts batches of proton from the $8 \mathrm{GeV} / c$ Booster accelerator to accelerate to $120 \mathrm{GeV} / c$. To create NuMI Neutrino Beam, primary $120 \mathrm{GeV}$ protons are extracted from the Main Injector. These protons are extracted from the MI in a $10 \mu s$ in spill and bent downward by 58 mrad to point at the Near and Far

Detector. Typically the extracted beam contains a total of $2.1 \times 10^{13}$ protons with a cycle per spill time of 2.2-2.4 s. The proton beam is focused on a carbon target which was stable to within $\pm 0.1 \mathrm{~mm}$ and the area of the beam-spot varied within the range 

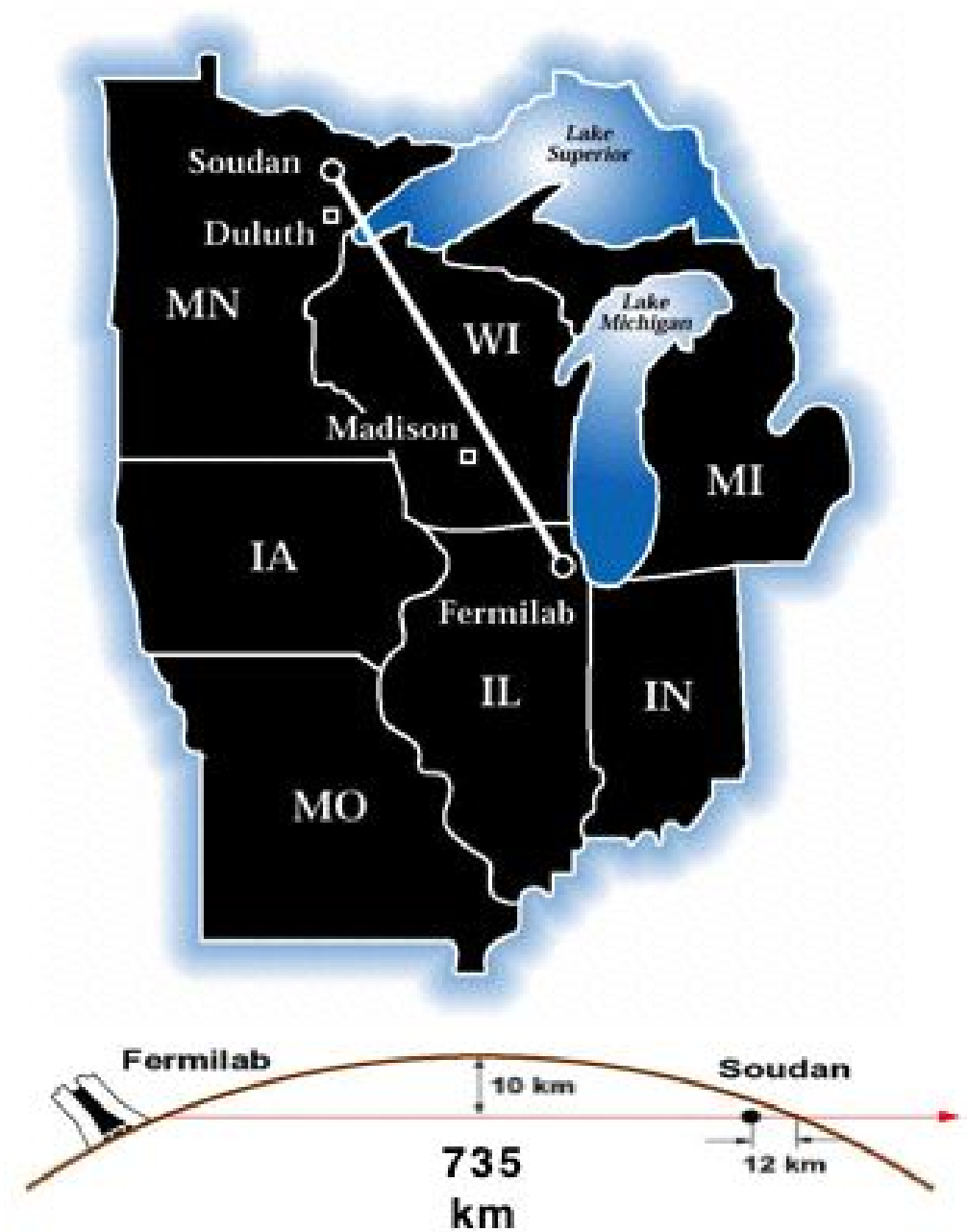

FIG. 13: Overview of MINOS experiment. Overview of MINOS experiment [44, 45]. 
$3.3-4.5 \mathrm{~mm}^{2}$. High energy collisions with the target create many particles, mostly $\pi^{ \pm}$ and $K^{ \pm}$. These particles are focused by a magnetic horn and travel down a $675 \mathrm{~m}$ decay pipe, where these beam particles are allowed to decay into muon and muon neutrinos. Remaining hadrons and muons are stopped in a concrete absorber and the subsequent rock to yield a beam of muon neutrinos. Ionization chambers are placed upstream of the absorber to monitor the beam by measuring the flux of hadrons and muons. Figure 14 shows a schematic of the NuMI beamline.

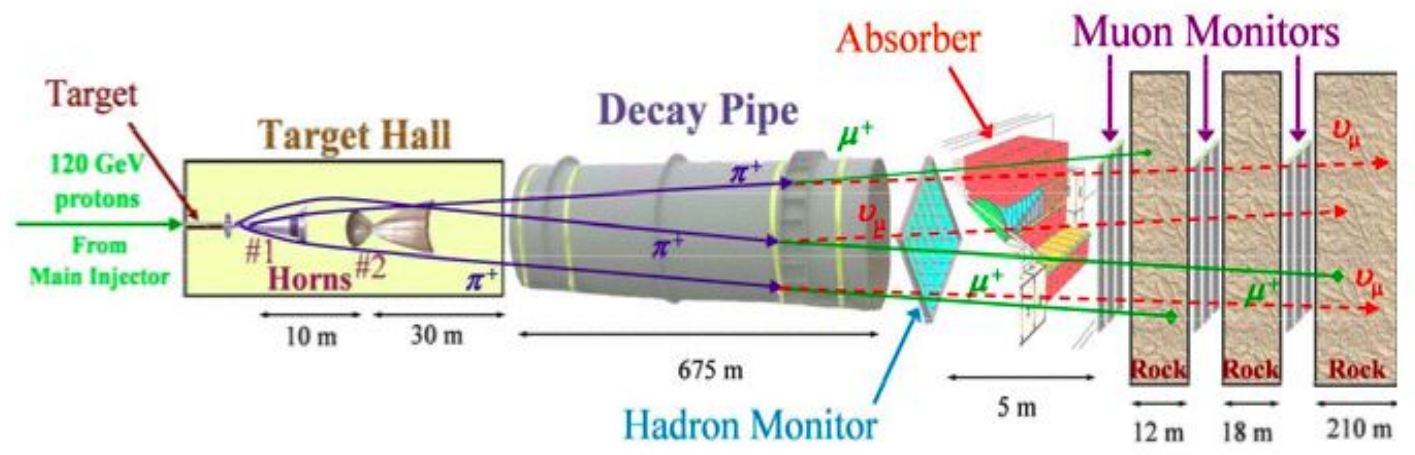

FIG. 14: Schematic view of the NuMI beamline. Protons from the MI enter from the left and the produced neutrinos exit to the right $[44,45]$.

Beam Configuration : Peak energy of neutrino beam can be configured from $3 \mathrm{GeV}$ to $10 \mathrm{GeV}$ depending on the distance between the target position and the first horn. Relative longitudinal positions of two horns and the target are adjusted to optimize the momentum focus for pions and kaons. If the target distance is increased, then the beam energy is increased. In NuMI, this distance can be remotely controlled and continuously varied from $0.1 \mathrm{~m}$ up to $2.5 \mathrm{~m}$. Figure 15 shows Near Detector neutrino beam energy spectra for different beam configurations. These beam energy configurations are only sensitive to high- $\Delta m^{2}$ values. In the beam spectrum below 
the $6 \mathrm{GeV}$, the beam is made up of a $92.9 \% \nu_{\mu}$, with a $5.8 \%$ component of $\overline{\nu_{\mu}}$ and a $1.3 \%$ component of $\nu_{e}+\overline{\nu_{e}}$.

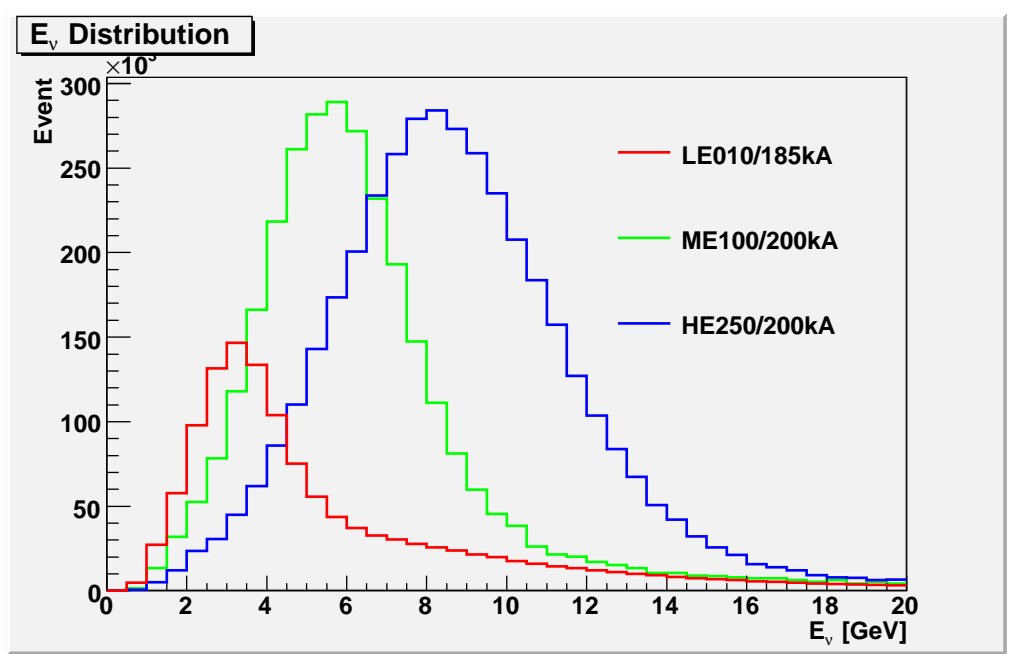

FIG. 15: NuMI beam configurations. The Near Detector MC spectra for low, medium and high energy beam configurations. If the distance between the target position and first horn is increased, the peak energy position is shifted to higher energy. 


\section{B. The MINOS Detectors}

The MINOS detectors are magnetized steel/scintillator tracking-sampling calorimeters. $2.54 \mathrm{~cm}$ thick steel plates are used as absorber material, while the active layers of each detector are made up of $1 \mathrm{~cm}$ thick plastic scintillator strips. The strips are $4.1 \mathrm{~cm}$ wide and are arranged side-by-side to form planes. These scintillator planes are mounted on the steel planes and rotated $90^{\circ}$ with respect to previous plane to construct three dimensional tracking images. Each scintillator strip has a wave length shifting(WLS) fiber along its length. Light produced by a particle traveling through the scintillator strips is captured by the WLS and transported a multi-anode photomultiplier tubes (PMT). A typical minimum ionizing particle produces $\sim 6$-7 photoelectrons in a PMT.

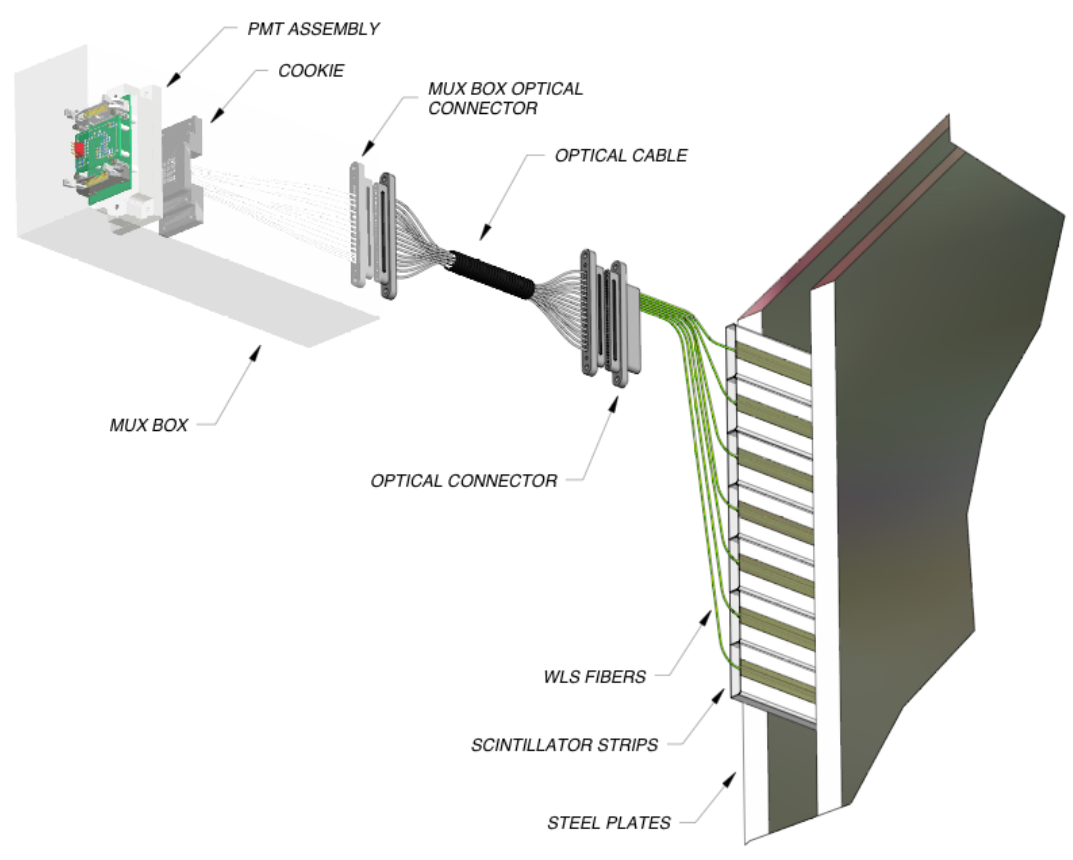

FIG. 16: Schematic view for one layer of scintillator plane in the detector [44]. 
The 0.98 kton Near Detector is located only $1.0 \mathrm{~km}$ away from the target position and has been set up to study the systematic uncertainties of the beamline and to extrapolate the unoscillated spectrum to the Far Detector. Neutrino oscillations occur as they travel through space, however since the path length is so short here, there is no chance for us to observe the oscillated neutrino events in the Near Detector.

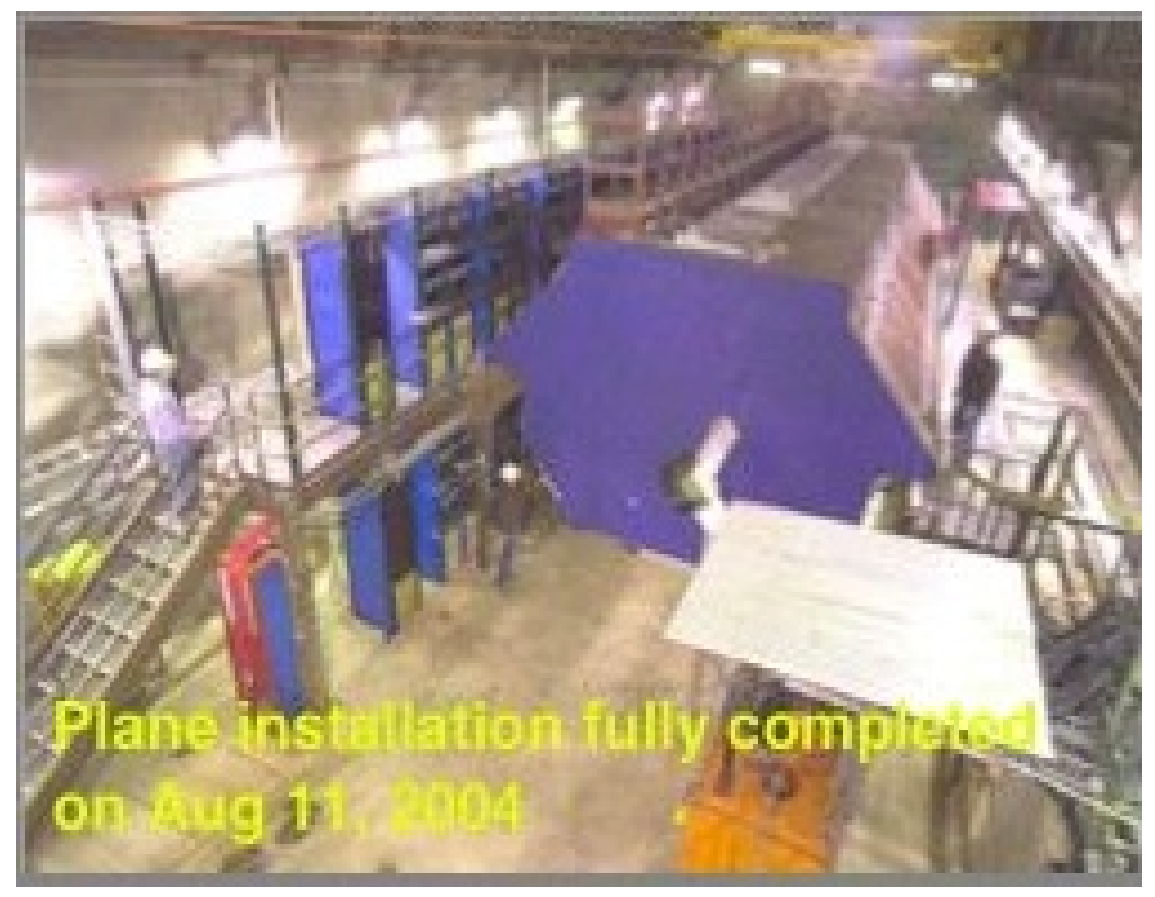

FIG. 17: Photograph of the Near Detector. The Near Detector [44, 45] located $104 \mathrm{~m}$ underground and consists of 282 irregular $4 \times 6 \mathrm{~m}^{2}$ octagonal steel and 153 scintillator planes read out at via a WLS fiber to one pixel of a Hamamatsu Multiplex M64 PMT and the Fast QIE electronics system that is capable of continuously recording signals without dead time throughout the $\sim 10 \mu$ s beam spill. A toroidal magnetic field is a toroidal field produced by a running a current of $185 \mathrm{kA}$ through the center of the detector producing an average value of $1.2 \mathrm{~T}$. 
The 5.4 kton Far Detector located $735 \mathrm{~km}$ away from the target position has been set up to study the oscillation parameters of the beam neutrinos after traveling this distance. By measuring the "oscillated" beam spectrum, we are able to find the neutrino's mass and mixing. To select beam related neutrino events, GPS-time stamping has been used to sync Far data to Near and beam data.

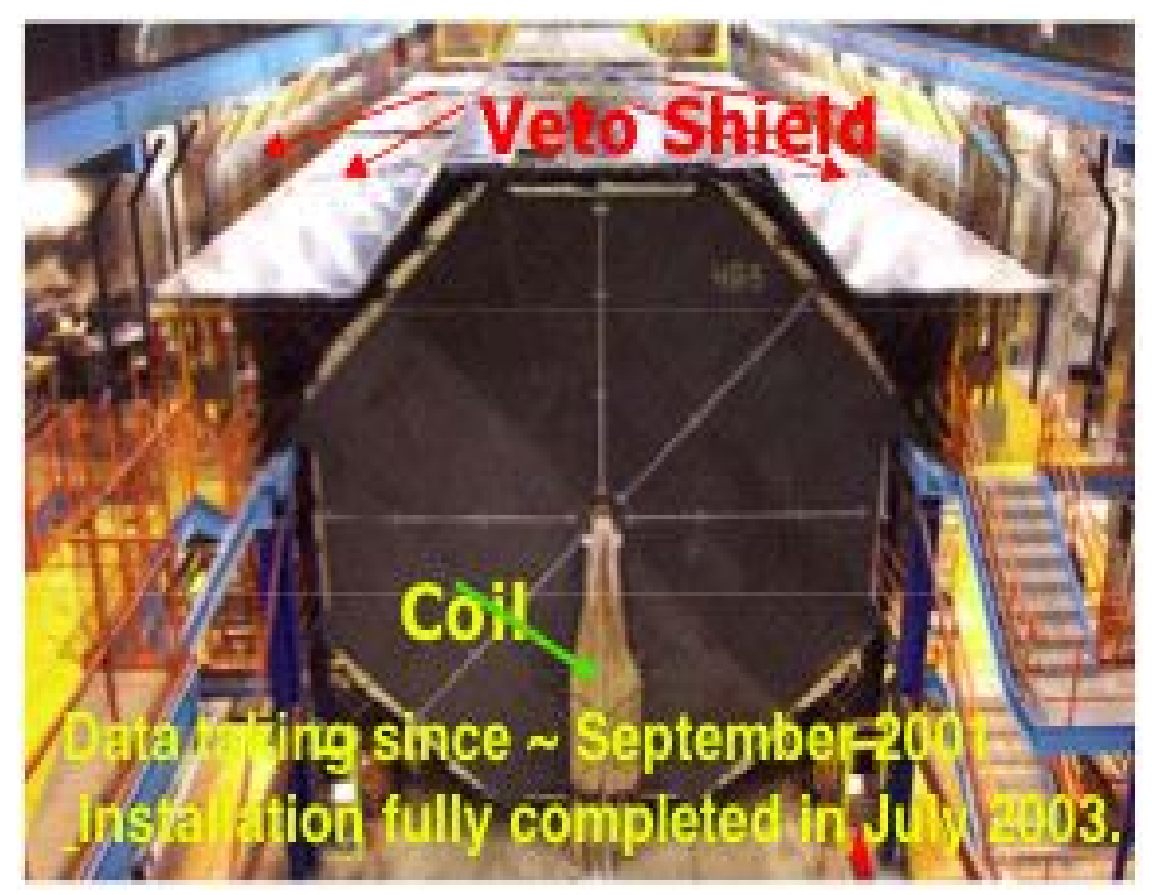

FIG. 18: Photograph of the Far Detector. The Far Detector [44, 45] is located $705 \mathrm{~m}$ beneath the surface and consists of 484 octagonal $8 \mathrm{~m}$ wide steel and scintillator planes read out at both ends via Hamamatsu Multiplex M16 PMTs and the VA electronics systems. The $40 \mathrm{kA}$ current carried by the coil running through the center of the detector induces the magnetic field of $1.17 \mathrm{~T}$. Veto shields are installed on the top of the detector to remove cosmic muon events. 


\section{Event Reconstruction}

MINOS classifies neutrino interactions based on the level of energy deposition, topology and timing of scintillator strip hits in each event. $\mathrm{CC} \nu_{\mu}$ interactions are characterized by the presence of a track associated with muon produced in the neutrino interactions. Often a cluster of hits near the interaction vertex can be identified as the result of the accompanying hadronic shower. Total energy of the $\mathrm{CC} \nu_{\mu}$ interactions is reconstructed as the sum of the muon momentum and the energy of the hadronic shower. The muon momentum is measured via the range and curvature of the muon track if the track stops inside of the detector and exits the detector. On the other hand, $\mathrm{NC} \nu_{\mu}$ interactions are characterized by the absence of a muon track and composed only with hadronic shower. Figure 19 shows the simulated events for the $\mathrm{CC}$ and $\mathrm{NC} \nu_{\mu}$ interactions in the Near Detector.

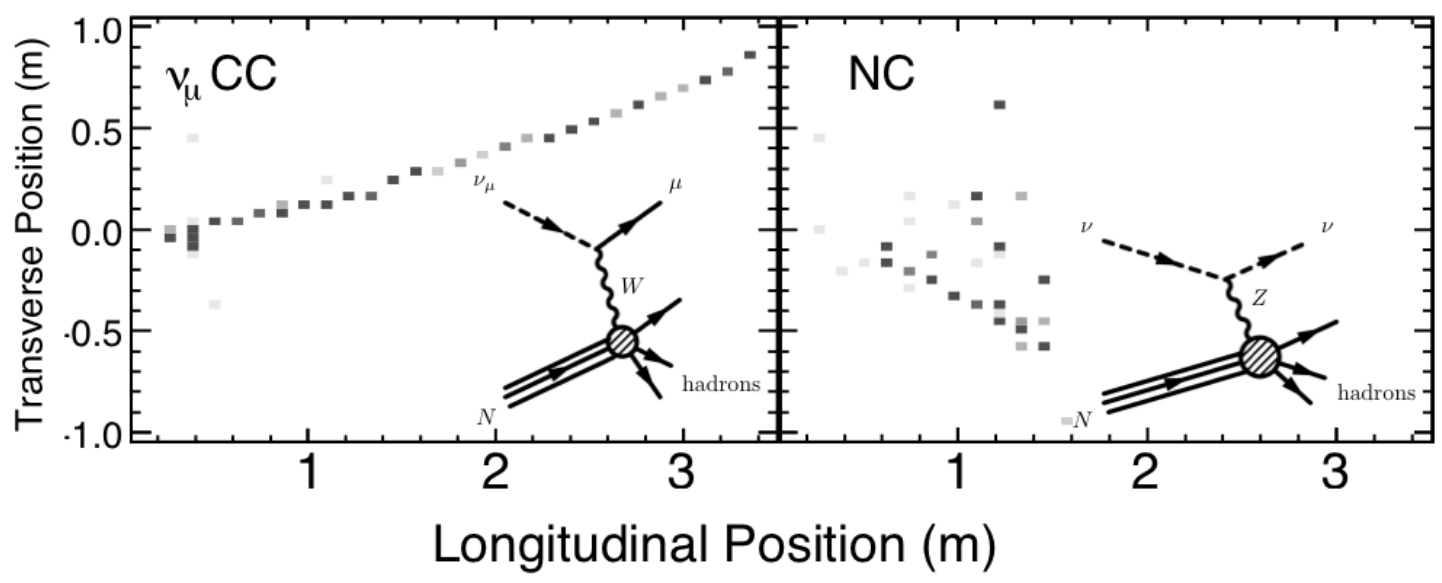

FIG. 19: 2-dimensional views of the $\mathrm{CC}$ and $\mathrm{NC} \nu_{\mu}$ interactions. The 2-dimensional views of the simulated events for the $\mathrm{CC}$ and $\mathrm{NC} \nu_{\mu}$ interactions in the Near Detector. The shaded rectangular boxes indicate relative energy deposited in the strip. These figures are referenced from $[44,45,82]$. 


\section{Detector Calibration}

Since one of the requirements in making these measurements is an accurate determination of the energies of the observed neutrinos, the relative energy scale between Near and Far Detectors is required to match within 2\%. The detector's responses are calibrated with a Light-Injection(LI) system, radioactive sources and cosmic ray muons. Each detector is calibrated in a multi-stage procedure that converts the raw photomultiplier signal $Q_{\text {raw }}(i, t, x)$ measured by channel $i$ at time $t$ for an energy deposition at position $x$ into a fully corrected signal $Q_{c o r r}$. Calibration constants are generated in each calibration procedure. The full correction is achieved by taking the product of raw signal and the constants from each stage. It is written in the form of $Q_{\text {corr }}=Q_{\text {raw }}(i, t, x) \times D(t) \times U(i) \times A(i, x) \times E$. The drift calibration $D(t)$ is performed using LI system. Light generated by LED are distributed through the WLS fibers to all PMT channels, to determine the PMT gains and the tracking gain over time. The uniformity correction $U(i)$ is performed using cosmic ray muons to normalize the output of each scintillator strip. The attenuation correction $A(i, x)$ is performed using a radioactive source to map out the response of each scintillator module at individual position and strip. Finally the absolute energy scales $E$ for Near and Far detectors are established by using stopping muons, to convert their signals to be comparable to each other in units of average light produced by a muon with energy between 0.5 to $1 \mathrm{GeV}$. 


\section{CHAPTER IV}

\section{ANALYSIS}

\section{A. Motivation}

The SK experiment shows that atmospheric neutrinos are maximally oscillated at $L / E \sim 500 \mathrm{~km} / \mathrm{GeV}$. The best fit value of the neutrino mass differences is $\Delta m^{2} \sim$ $2.47 \times 10^{-3} \mathrm{eV}^{2}$ for the maximal mixing. When the long-baseline neutrino oscillation experiments are organized to provide a test of the SK results using man made muon neutrino beam, the beam energy spectrum is generally configured to peak at $E_{\nu}^{\max } \sim$ $L_{0} / 500 \mathrm{GeV}$ where $L_{0}$ is the fixed distance that beam neutrinos travel through space. In principle, if $E_{\nu}<E_{\nu}^{\max }$, then it is sensitive to constrain lower $\Delta m^{2}$ region than the estimated value. On the other hand, higher $\Delta m^{2}$ can be constrained if $E_{\nu}>E_{\nu}^{\max }$.

Two beam-based experiments are currently operating in the world; the $\mathrm{K} 2 \mathrm{~K}$ and MINOS experiments. The first result for the beam neutrino oscillation measurement associated to the 2-3 mixing sector was made in the K2K experiment in Japan $[71,51]$. The distance that these neutrino travels through space is $L_{0}=250 \mathrm{~km}$. The beam energy is configured at the maximally oscillated position of $E_{\nu}^{\max } \sim 0.5 \mathrm{GeV}$ and shown in Figure 20 left. However, MINOS CC energy spectrum is configured to peak at energy above the maximal position. The appropriate beam energy configuration for MINOS distance is around $E_{\nu}^{\max } \sim 1.47 \mathrm{GeV}$. However, the LE MINOS energy spectrum is peaked near $3 \mathrm{GeV}$. As a result, the event detection rate is high above 1.47 GeV and it is sensitive to constrain high- $\Delta m^{2}$ region, but statistics are poorer below $1.47 \mathrm{GeV}$. Oscillation contours in Figure 6 shows significant improvement at the higher region, compared to the K2K contour. What can MINOS do to improve the measurement at low- $\Delta m^{2}$ region? Selecting the QEL events may help to improve 

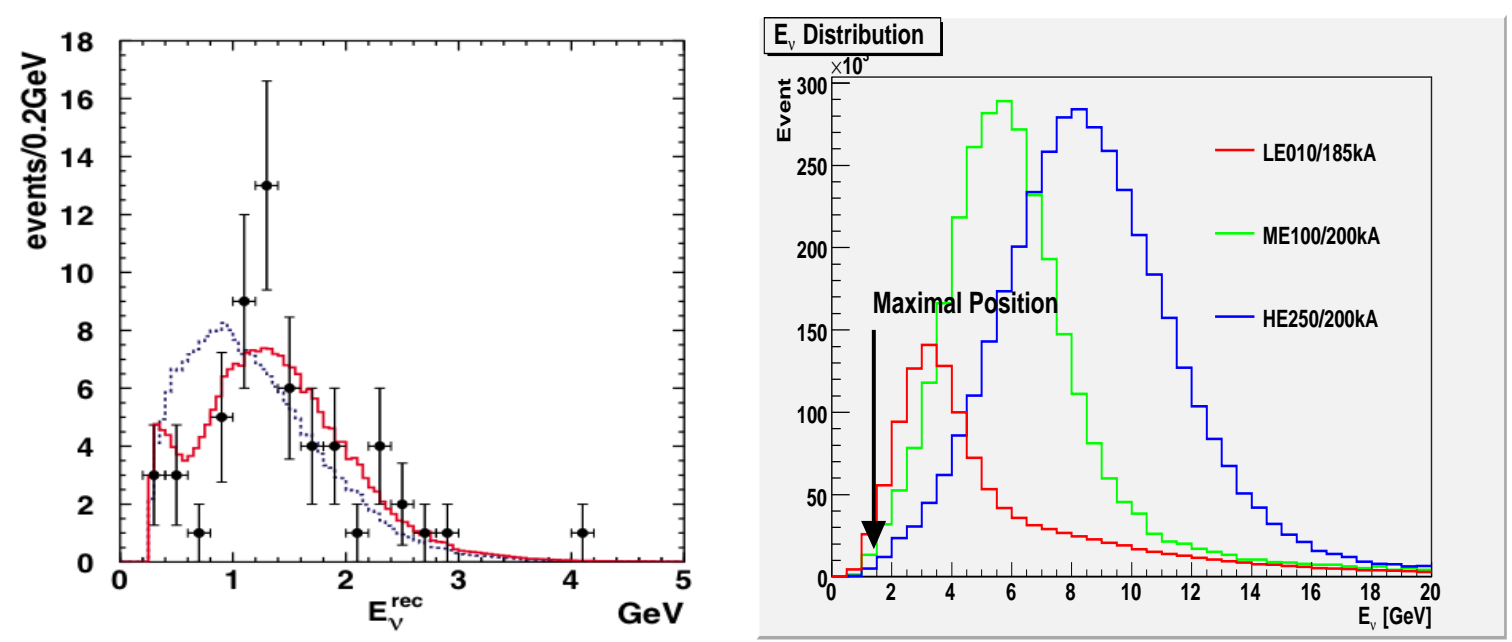

FIG. 20: Results from K2K and MINOS experiments. (Left) Energy spectrum is configured below $1 \mathrm{GeV}$ for $\mathrm{K} 2 \mathrm{~K}$ experiment [71, 51]. (Right) MINOS energy is peaked out of the maximal position [9].

the measurement for following two reasons:

(1) A QEL interaction is the dominant physical process below the maximally oscillated position. Selected events for the $\mathrm{CC}$ analysis are mixture of three types of $\mathrm{CC}$ neutrino interactions; quasi elastic (QEL), resonance (RES) and deep inelastic scattering (DIS). The QEL interaction is the nearly elastic process that changes quark flavor when a neutrino interacts with a neutron. The process is shown as $\nu_{l}+n \rightarrow l^{-}+p$. Similarly RES interaction can be formulated in $\nu_{l}+n \rightarrow l^{-}+\pi^{+}+p$, but $W^{+}$boson is resonated to pion mass and an extra charged pion can be produced in final states of interaction. Lastly, DIS interaction is the high energy interaction process that can break the internal nucleon structure and produce various hadronic particles in the final state. The neutrino cross section is shown as a function of neutrino energy in Figure 21. The QEL interactions are dominant in the energy region important to 
improve the low- $\Delta m^{2}$ region.

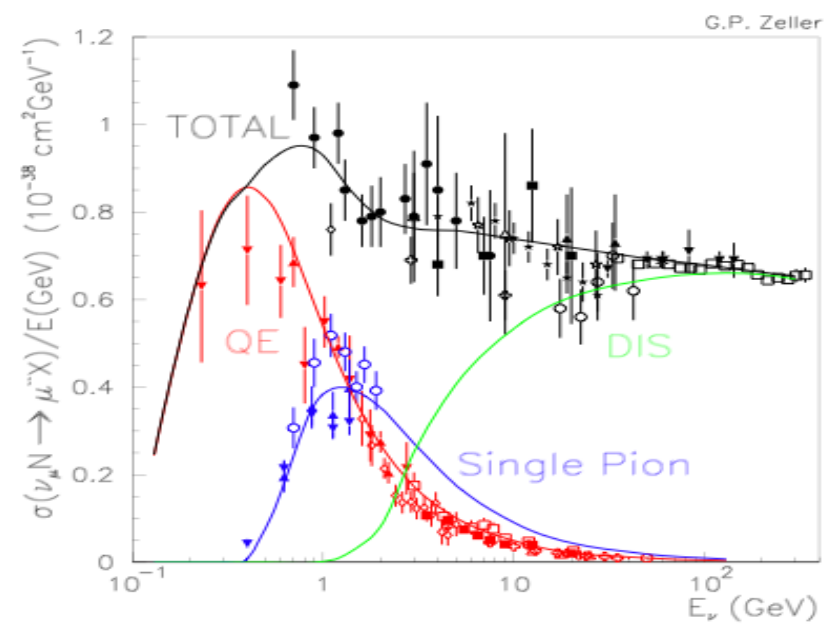

FIG. 21: Neutrino cross section. Neutrino cross section for QEL (red), RES (blue) and DIS (green) interactions shows as a function of neutrino energy. The figure is referenced from [72].

(2) Reconstruction of CC neutrino energy is done by just making sum of muon and hadronic shower energy. MINOS detectors are specifically designed to detect high energy muon events and are able to measure the muon energy with a resolution of $2 \%$. When most of the incoming muon neutrino energy is carried out by outgoing muon, the muon leaves a long track in the detector. If the track were short, then the energy is mostly converted into hadronic shower and various hadronic particles are created at final state. Hence the oscillation measurement is largely influenced by the large uncertainty of shower energy reconstruction. However, better neutrino energy reconstruction can be accomplished through the QEL interaction due to there being no missing energy. A muon and a proton are only particles in the QEL final state. QEL energy reconstruction only depends on muon energy and angle. Thus in 
principle it is possible to perform more sensitive oscillation measurement with QEL events rather than $\mathrm{CC}$ events.

\section{B. The Near Detector Analysis}

The Near Detector was built for the purpose of studying systematic variance and covariance of neutrino flux from the NuMI beamline, detector response and the neutrino interaction cross-sections. Understanding details of the beam production and neutrino-nucleon scattering models will be very important to do the QEL oscillation analysis, especially for predicting the unoscillated Far Detector spectrum. The Near Detector has collected a large sample of relatively high energy CC events and observed the large discrepancy between data and nominal MC in the CC spectra shown in Figure 22. The discrepancy can be separated into two different sections; high and low energy regions dominant to the DIS and QEL/RES event interactions. The MINOS Detectors are well designed and simulated to measure high energy muons. The primary cause of the high energy discrepancy is from mis-modeling of the neutrino flux from the beamline, rather than the detector and cross section effects. The best fit values of the optical and hadron production parameters [73] are applied in Figure 22. Possible sources of the low energy discrepancy are from the mis-modeling of the remaining QEL/RES cross sections and the detector response to low energy hadrons.

\section{Model Adjustment : QEL Cross Sections}

The charged current weak interaction is a theory of quark and lepton coupling to $W^{ \pm}$boson via vector and axial vector vertex factors that allows conserving neutrino helicity. The weak vertex is generally treated as a point-like interaction that quark and/or lepton are mediated through $W^{ \pm}$bosons and written in the form of $\gamma^{\mu}\left(1-\gamma^{5}\right)$. 

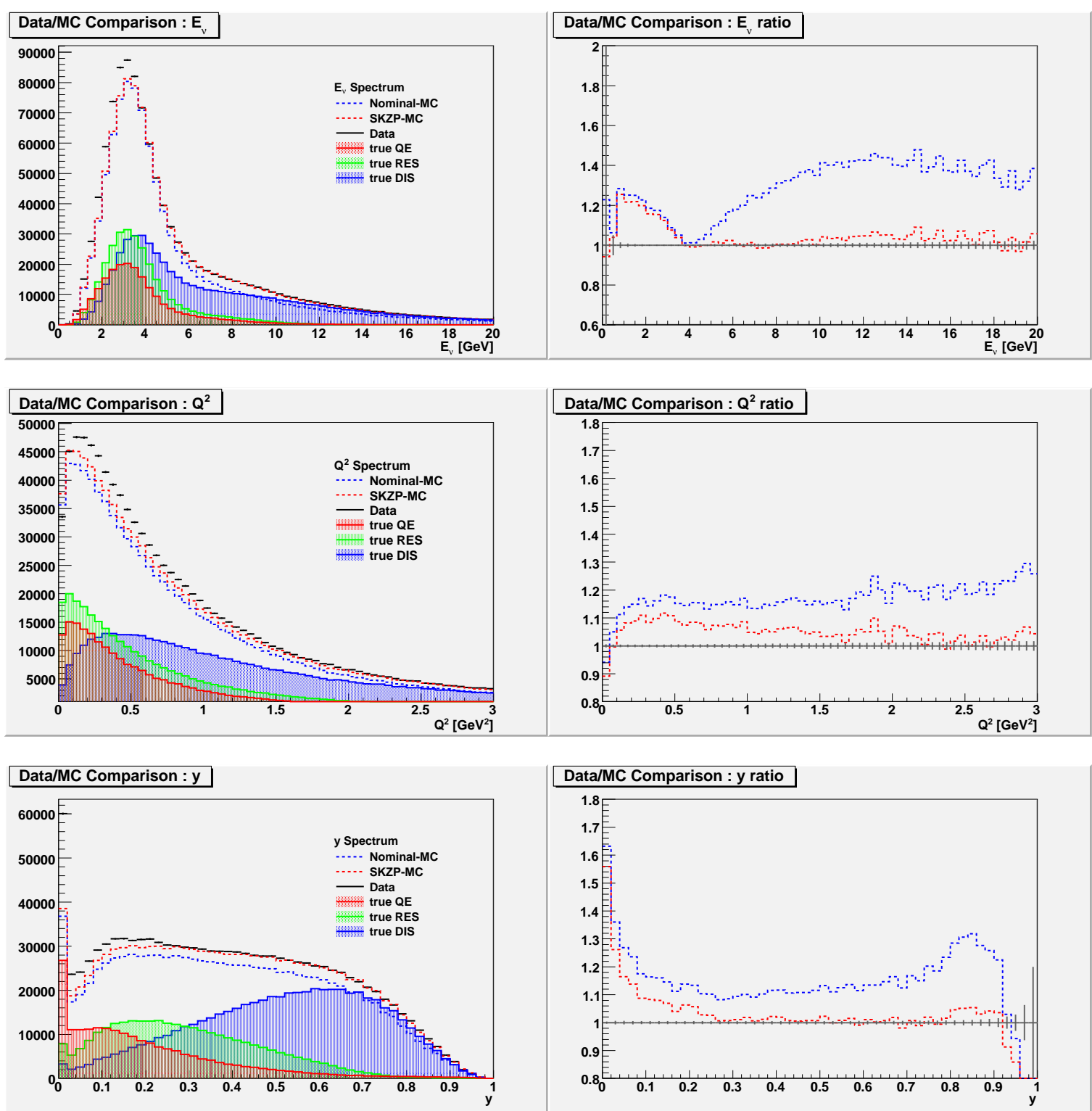

FIG. 22: CC-like event distributions. CC-like event distributions and the ratio spectra of MC to data are shown for the 1.27 and the $1.47 \times 10^{20}$ POT data and MC sets from the low energy beam configuration (LE-010,185kA). Blue and red dashed lines are for the nominal and SKZP reweighted MC. The $E_{\nu}^{C C}, Q^{2}$ and $y$ distributions and data/MC ratios are shown in the top, middle and bottom figures. 
The first term yields vector coupling. The second term is the axial vector coupling, which is maximally bound to violate parity conservation. However, real neutrino scattering from nucleons is not a point-like interaction. To take into account of the nucleon's internal structure, the point-like vertex factor can be reformulated to

$$
\begin{aligned}
\text { vector : } & \gamma^{\mu} \longrightarrow \gamma^{\mu} F_{V}^{1}\left(q^{2}\right)+\frac{i \sigma^{\mu \nu} q_{\nu}}{2 m_{N}} \xi F_{V}^{2}\left(q^{2}\right) \\
\text { axial vector }: & \gamma^{\mu} \gamma^{5} \longrightarrow \gamma^{\mu} \gamma^{5} F_{A}\left(q^{2}\right)
\end{aligned}
$$

Unlike electric charge, weak charge is not modified by strong interaction within the nucleon structure. The axial part of the weak charge is not conserved while vector part is protected in the conserved vector current hypothesis $(\mathrm{CVC}) . F_{V}^{1}$ and $\xi F_{V}^{2}$ are so called the Dirac and Pauli electromagnetic isovector form factors, which formulated in terms of Sachs vector form factors. The Sachs form factors $G_{E}^{P}\left(q^{2}\right), G_{E}^{N}\left(q^{2}\right)$, $G_{M}^{P}\left(q^{2}\right)$ and $G_{M}^{N}\left(q^{2}\right)$ are related to the electric and magnetic moment distributions and are well measured by electron scattering experiments. Finally the axial vector form factor is approximately a dipole under the assumption that an axial part of the weak charge is symmetrically distributed with exponential function, $\rho_{A}(\vec{r})=e^{-M|\vec{r}|}$. It is written in form of

$$
F_{A}\left(q^{2}\right)=F_{A}(0) \int \rho_{A}(\vec{r}) e^{i \vec{q} \cdot \vec{r}} d \vec{r}=\frac{F_{A}(0)}{\left(1-q^{2} / M_{A}^{2}\right)^{2}}
$$

where $F_{A}(0)=1.2720 \pm 0.0018$ and $M_{A}$ is the axial vector mass. $F_{A}(0)$ is an axial part of the weak charge correction terms when $q^{2}=0$.

The scattering amplitude (or matrix element) can be written in the form of

$$
-i \mathbf{M}=\left[\overline{u\left(\mu^{-}\right)} \frac{-i g_{w}}{2 \sqrt{2}} \gamma^{\mu}\left(1-\gamma^{5}\right) u\left(\nu_{\mu}\right)\right] \frac{i g_{\mu \nu}}{M_{W}^{2}}\left[\overline{u(p)} \frac{-i g_{w}}{2 \sqrt{2}} \Gamma_{C C}^{\nu}\left(q^{2}\right) \cos \theta_{C} u(n)\right]
$$


where $\Gamma_{C C}^{\nu}\left(q^{2}\right)=\gamma^{\mu}\left(F_{V}^{1}\left(q^{2}\right)-\gamma^{5} F_{A}\left(q^{2}\right)\right)+\frac{i \sigma^{\mu \nu} q_{\nu}}{2 m_{N}} \xi F_{V}^{2}\left(q^{2}\right)$ and $g_{w}, M_{W}$ and $\cos \theta_{C}$ are the weak coupling constant, the charged $\mathrm{W}$ boson mass and Cabbibo mixing angle respectively. The first bracket shows that the muon neutrino couples to its own charged lepton via a point-like vertex factor. $\Gamma_{C C}^{\nu}\left(q^{2}\right)$ in second bracket is the form factor that neutron couples to proton. Middle term is $W^{ \pm}$boson propagator. Using the amplitude and Feynman rules for neutrino scattering, the differential cross section for the QEL interaction as a function of $Q^{2}$ is formulated with the relativistic Fermi gas (RFG) model ${ }^{4}$ and simplified in the form of

$$
\frac{d \sigma}{d\left|q^{2}\right|}=\frac{m_{N}^{2} G_{F}^{2} \cos ^{2} \theta_{c}}{8 \pi E_{\nu}^{2}}\left[A\left(q^{2}\right)-B\left(q^{2}\right) \frac{s-u}{m_{N}^{2}}+C\left(q^{2}\right) \frac{(s-u)^{2}}{m_{N}^{2}}\right]
$$

where $s$ and $u$ are Mandelstam variables, $s-u=4 m_{N} E_{\nu}+q^{2}-m_{l}^{2}, m_{l}$ is the outgoing lepton mass and $m_{N}$ is the target nucleon mass. $A\left(q^{2}\right), B\left(q^{2}\right)$ and $C\left(q^{2}\right)$ are expressed in term of the vector and axial vector form factors. Details are described in Ref. [74].

Currently the axial vector form factor of the nucleon and QEL cross section is not well understood at lower energies with large uncertainties at level of $20 \%$ to $30 \%$. A number of the QEL $M_{A}$ (axial vector mass see Equation (4.1)) measurements were performed using various target materials, but these measurements using predominantly deuterium as a target material were limited by low statistics and encountered some difficulties describing their data at low momentum transfer $Q^{2}$, where it is largely influenced by the nuclear effects. The MINOS experiment uses an iron target and has

\footnotetext{
${ }^{4}$ The theoretical concepts of Fermi gas is applied to simulate the nuclear effects in the neutrino-nuclear scattering. Protons and neutrons are considered as moving quasi-freely within nuclear volume and obeying the Fermi-Dirac statistics leading to the Pauli exclusion principle. These particles are distinguishable fermions and situated in two separate finite potential wells. The depth of the potential wells is given by $V_{0}=E_{F}+\epsilon_{B}$, where $\epsilon_{B}$ and $E_{F}$ are the nuclear binding energy and the Fermi energy of the highest occupied nucleon level.
} 

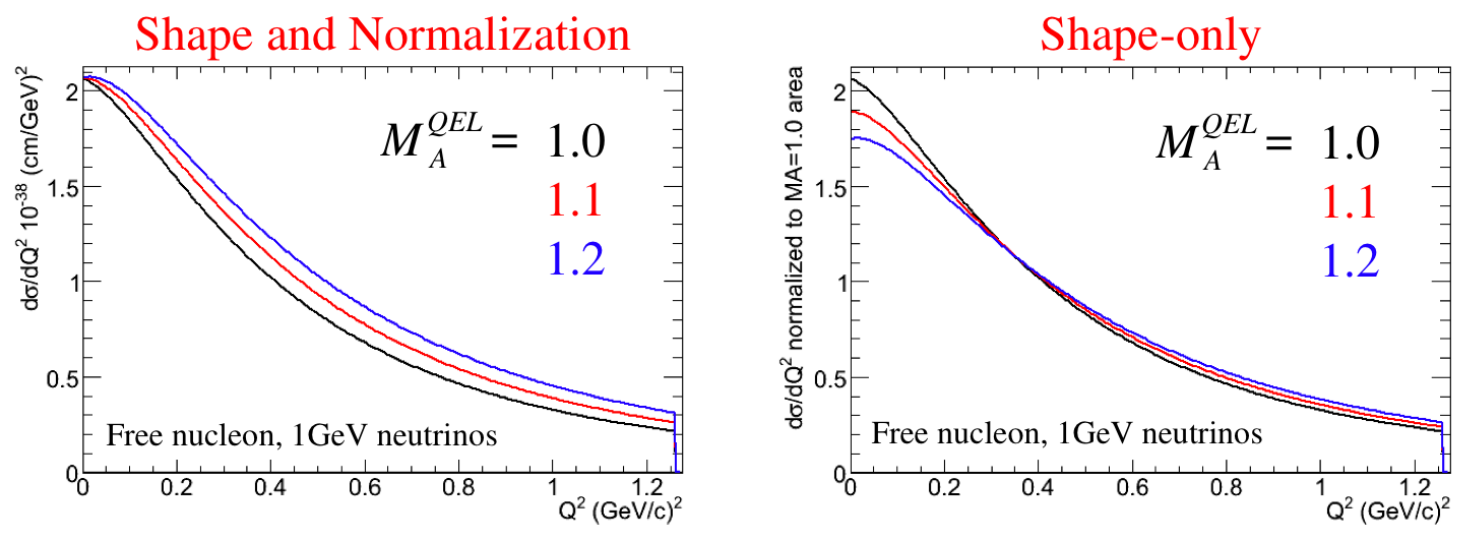

FIG. 23: Effect of changing $M_{A}^{Q E L}$-scae for free nucleon QEL interaction cross section. Effect of changing $M_{A}^{Q E L}$ scale for free nucleon QEL interaction cross section. Shape and normalization of the $Q^{2}$ distribution are influenced by changing the $M_{A}^{Q E L}$ scale. Left and right figures are scaled by the POT counting and the area of $M_{A}=1.00 \mathrm{GeV}$. These curves are correspond to mono-energetic neutrinos at $1 \mathrm{GeV}$ and ignore nuclear effects. The high $Q^{2}$ cutoff is purely kinematic. These figures are referenced from $[78]$.

collected large samples of relatively high energy muon neutrino QEL events to date. The use of such events in tuning the cross section effects allows us to better predict the unoscillated spectrum at Far Detector. The Near Detector analysis not only fits the reconstructed $E_{\nu}$ distribution, but also the $Q^{2}$ distribution with the adjustable $M_{A}^{Q E L}$ parameter. Varying the $M_{A}^{Q E L}$ parameter significantly appears to disturb the shape and normalization of the $Q^{2}$ distribution. Figure 23 shows that the differential cross section is shown as a function of $Q^{2}$ for mono-energetic neutrino scattering off free nucleon using different $M_{A}^{Q E L}$ scale. Both of the normalization and shape of $Q^{2}$ distributions are clearly influenced as changing $M_{A}^{Q E L}$ scale. 


\section{QEL Selection : QEL Kinematic Correction}

Near Detector analysis is performed using the data set of $1.27 \times 10^{20}$ POT (cedar_phy version) during the period May 20, 2005 to Feb. 25, 2006. The cumulative distribution of the number of total POT is shown as a function of time or run number in Figure 24. POT counting is proportional in time. The Near Detector data is initially selected by using the CC-like event cuts. A maximum likelihood method is used for selecting the $\mathrm{CC}$ events. The particle identification parameter (PID) is developed to discriminate between CC- and NC-like events, using one or two dimensional probability density functions (PDF) such as track pulse height fraction, number of track-like planes, pulse height per track-like plane, goodness of muon track fit, reconstructed charge of track, reconstructed y, muon scattering angle and track length. These distributions are normalized to unity. A probability for each event being $\mathrm{CC}$ and $\mathrm{NC}$

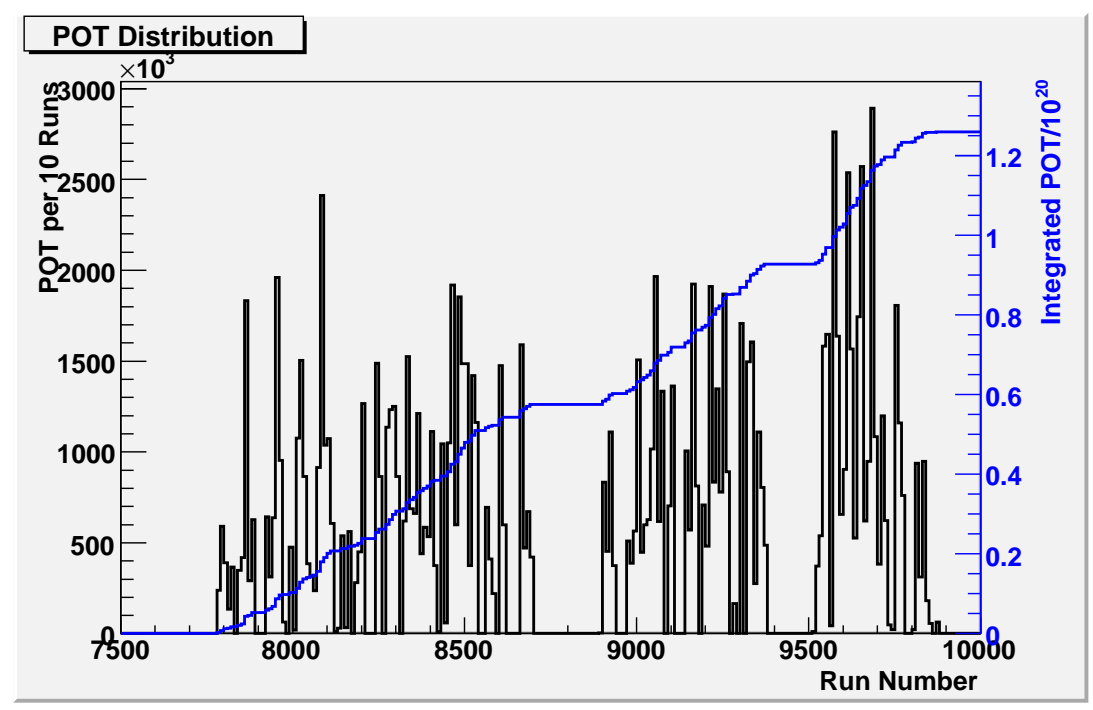

FIG. 24: Near Detector POT distribution. POT and integrated POT distributions are shown as a function of time or run number. 
event is given by the product of each PDFs and written in the form of $P_{C C}=\prod_{i} f_{i}(C C)$ and $P_{N C}=\Pi_{i} f_{i}(N C)$. The PID variable is constructed from those probabilities $P_{C C}$ and $P_{N C}$ as follows; $P I D=\frac{P_{C C}}{P_{C C}+P_{N C}}$. Finally $\mathrm{CC}$ events are selected at $P I D_{A B}>0.85$. More details are described in Ref. [75]. The PID distribution is shown on the left of Figure 25. Purity and efficiency for the initial cut is calculated as a function of neutrino energy and shown in right Figure 25. MC/data sets and initial CC-like event selection cuts are listed as follows;

- 2008 Standard CC fiducial cut

- Negative signed one track event $($ ntrack $=1$ and $q / p<0)$

- Track vertex difference between $\mathrm{U}$ and $\mathrm{V}$ planes $<6$

- Track end difference between $\mathrm{U}$ and $\mathrm{V}$ planes $<41$

- Track end planes $<270$

- CC-like cut : $P I D_{A B}>0.85$
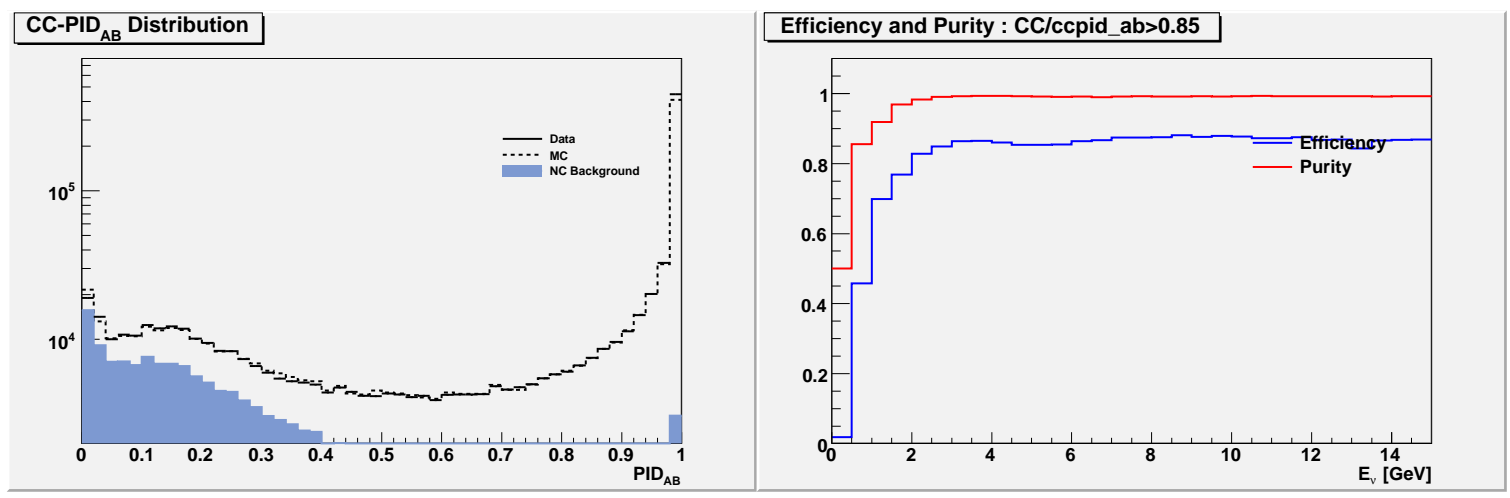

FIG. 25: Near Detector CC-like event distributions. (Left) PID (particle identification) is the parameter to discriminate between $\mathrm{CC}$ - and NC-like events. $\mathrm{MC}$ is normalized to the data event number. (Right) Efficiency and purity of the CC-like event selection are shown in blue and red lines. 
The QEL interaction is nearly elastic and conserved process, $\nu_{\mu}+n \rightarrow \mu^{-}+p$. The proton is the only hadronic particle in the final state and creates shower in the detector. Observation of proton events is one of the important key elements to construct the QEL event selection procedure. Typically a maximum likelihood method is used for selecting targeted events. Another PID parameter can be constructed to discriminate between QEL and NonQE events, using variables that represent differences between QEL events and the other interaction types as one or two dimensional PDFs. The method has the advantage for taking into account the different hadronic interaction types and multiplicities found in the various interaction modes, but it is sometimes difficult to find the appropriate parameters that characterize a proton's track from shower variables and is largely influenced by the uncertainties of the shower energy and angles. The basic properties of the shower resolutions are described as follows :

1. Most protons are recognized as showers in the detector, not as a track. A shower is mainly treated as a scaler energy value in the $\mathrm{CC}$ analysis. The internal form of shower distribution is just ignored.

2. According to the current study of hadronic interaction, hadronic shower energy is measured with a resolution of $10 \%$ and $0.050 \mathrm{GeV}$ in offset due to the intranuclear rescattering effects $[44,76,77]$. Hadronic momentum variance is parameterized as a function of true shower energy. Its minimum value is just the shower offset $[76,77]$ or the constant term of the parameterization. The uncertainty relation can be written in the form of

$$
\Delta p=\Delta E \frac{\partial p}{\partial E} \approx E_{\text {offset }}\left(1+\frac{1}{2}\left(\frac{m}{E}\right)^{2}+\cdots\right) \gtrsim E_{\text {offset }}
$$

where $E$ is hadron energy and larger than hadron mass of $m$ and $E_{\text {offset }}$ is 
the hadronic shower offset of about $0.050 \mathrm{GeV}$, estimated from the intranuclear rescattering effects. Thus the momentum variance is minimized with the shower energy offset.

3. Hadronic shower angle is a vector from event vertex to shower vertex that is formed by summing over the positions of the hits in the events. The angular resolution is determined by making a gaussian fit on the distributions of the angles between the true and reconstructed directions. It is well formulated in $\theta_{\text {res }}=16.67 / \sqrt{E}+12.15 / E[44]$. As the shower energy decreases to low energy, the shower direction is not very well defined and shower is most likely to form a bulk of energy around the event vertex. Thus the angular resolution is a $\pi$ at low energy.

QEL events are expected to reconstruct one or two tracks in a detector. Not only will the muon track be detected, but it is also possible to observe a proton track if the interaction is energetic enough to get out of the nucleus. Unfortunately the MINOS detectors are not well designed to observe hadron tracks. In principle, the proton is so heavy that its motion is classical which only allows it to create an energy deposition less than its own mass. QEL events are most likely to create no shower or a small amount of proton shower energy less than a few $\mathrm{GeV}$. Those signals are spherically distributed around event vertex and hence contain very little directional information. Thus a low energy shower can be typically recognized as a point in the MINOS detectors. To treat such shower variable in a simple manner, I would like to make the step-by-step shower corrections (See Appendix A.). Consider the internal form of shower energy spread that continuously transforms from a point or bulk to a track in minimum and maximum limits. The transformation of the internal shower spread is shown in Figure 26. 


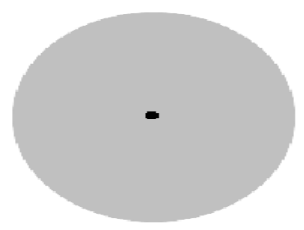

(a) Point

(b) Bulk

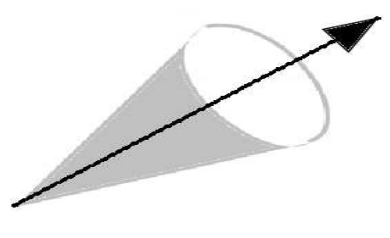

(c) Cone

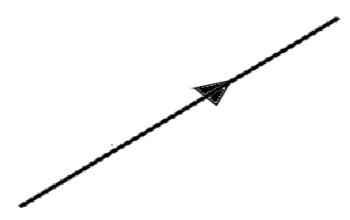

(d) Track

FIG. 26: Transformation of the internal shower spread. Shower is a point and track in minimum and maximum limits.

As a first step, assume that shower is a point-like variable at low energy. Prepare for two kinematic cuts to select QEL events. The first kinematic cut can be defined by shower energy less than $1 \mathrm{GeV}$. Shower energy distribution and cut are shown in Figure 27.

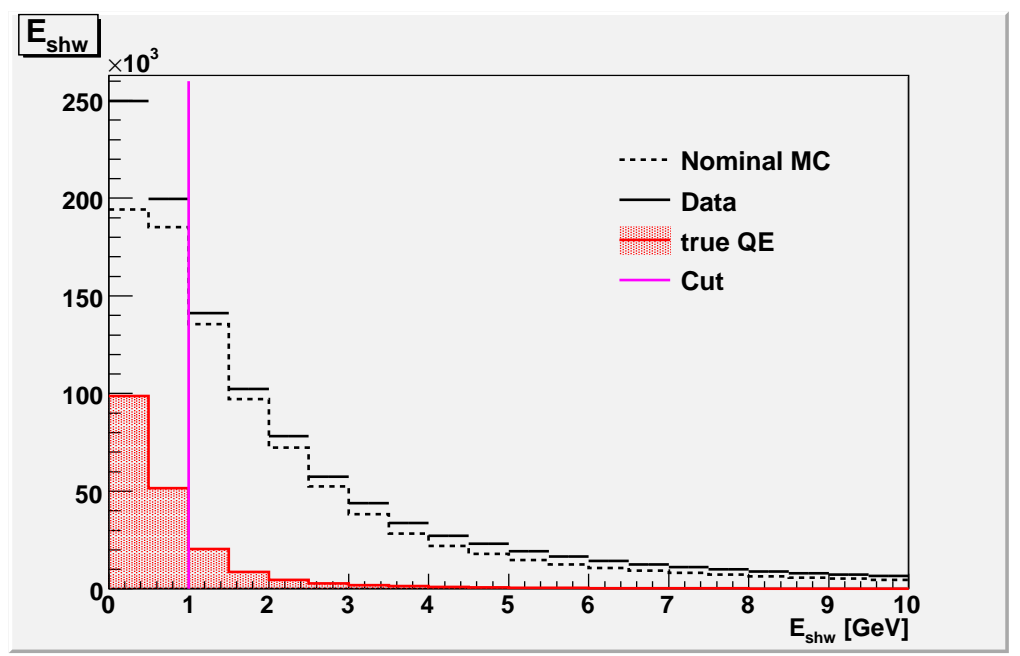

FIG. 27: Shower energy distribution. First kinematic cut of shower energy less than $1 \mathrm{GeV}$ is indicated in pink line. Red histogram shows true QEL event distribution. 
The second kinematic cut is the shower variance cut formulated using the conservation of energy law. Typically shower variance is calculated in position basis, but it will be reformulated in momentum basis for the future convenience. Details are described in Appendix A. Reconstruction procedure is described in following three steps :

Step 1. - Energy Reconstruction : QEL neutrino energy can be reconstructed in two different ways. One way is that the energy reconstruction only depends on muon variables, namely $Q E$ energy spectrum. QEL interaction is nearly energy conserved process that it can be only converted by simple four momentum vector calculation. The calculation of the neutrino energy can be simplified to the following

$$
E_{\nu}^{Q E}\left(E_{\mu}, \cos \theta_{\mu \nu}\right)=\frac{m_{n} E_{\mu}-m_{\mu}^{\prime 2} / 2}{m_{n}-E_{\mu}+p_{\mu} \cos \theta_{\nu \mu}}
$$

where $m_{\mu}^{\prime 2}=m_{\mu}^{2}-m_{p}^{2}+m_{n}^{2} . E_{\mu}, \cos \theta_{\nu \mu}, m_{\mu}, m_{p}, m_{n}$ and $E_{s h w}$ are the reconstructed muon energy, angle and mass, proton and neutron masses and shower energy. Its derivation is described in Appendix C. The second method is to reconstruct the

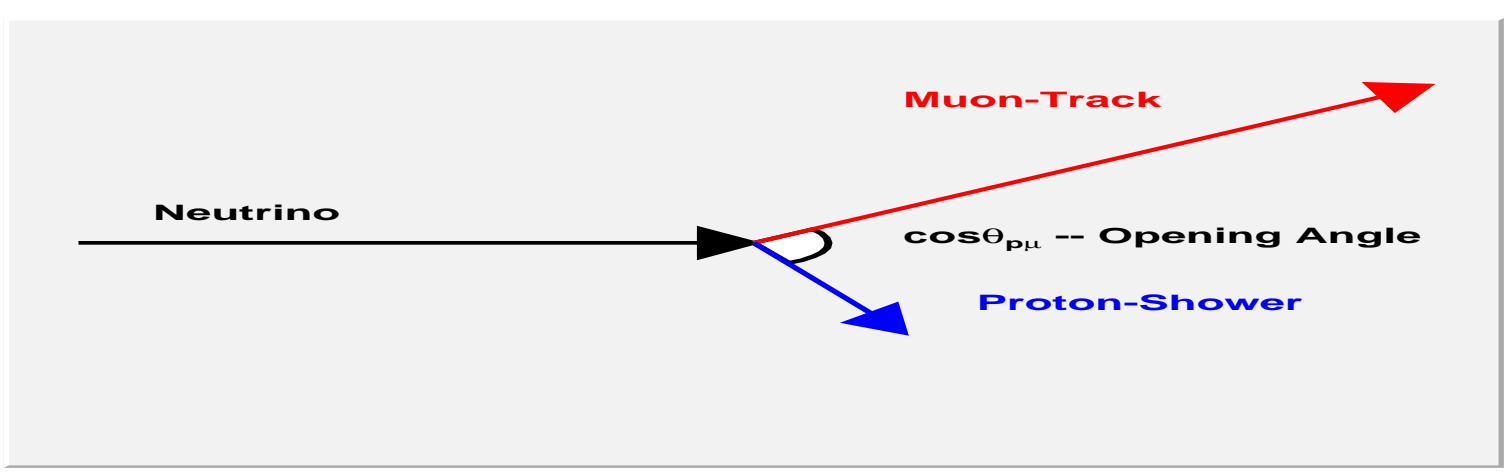

FIG. 28: Schematic view of QEL neutrino interaction. Incident neutrino, outgoing muon and proton are shown in black, red and blue arrows. 
neutrino energy by using muon and shower variables together, namely $C C Q E$ energy spectrum. Neutrino events are identified not only by muon variables, but also shower variables. First use the QEL energy formula if an event has no shower. The CCQE energy can be also converted by the standard CC neutrino energy reconstruction if an event has shower. The CCQE neutrino energy is written as

$$
E_{\nu}^{C C Q E}\left(E_{\mu}, \cos \theta_{\mu \nu}, E_{s h w}\right)=\left\{\begin{array}{ccc}
E_{\nu}^{Q E}\left(E_{\mu}, \cos \theta_{\mu \nu}\right) & \text { if } & N_{s h w}=0 \\
E_{\mu}+E_{s h w} & \text { if } & N_{s h w}>0
\end{array}\right.
$$

Step 2. - Proton-Muon Opening Angle : The opening angle is simply derived by performing four momentum vector calculation, ignoring effective binding energy term for Fermi gas model. The formula is written in the form of

$$
\cos \theta_{\mu p}=\frac{E_{\mu} E_{p}-E_{\nu} m_{p}+\left(m_{\mu}^{2}+m_{p}^{2}-m_{n}^{2}\right) / 2}{p_{\mu} p_{p}}
$$

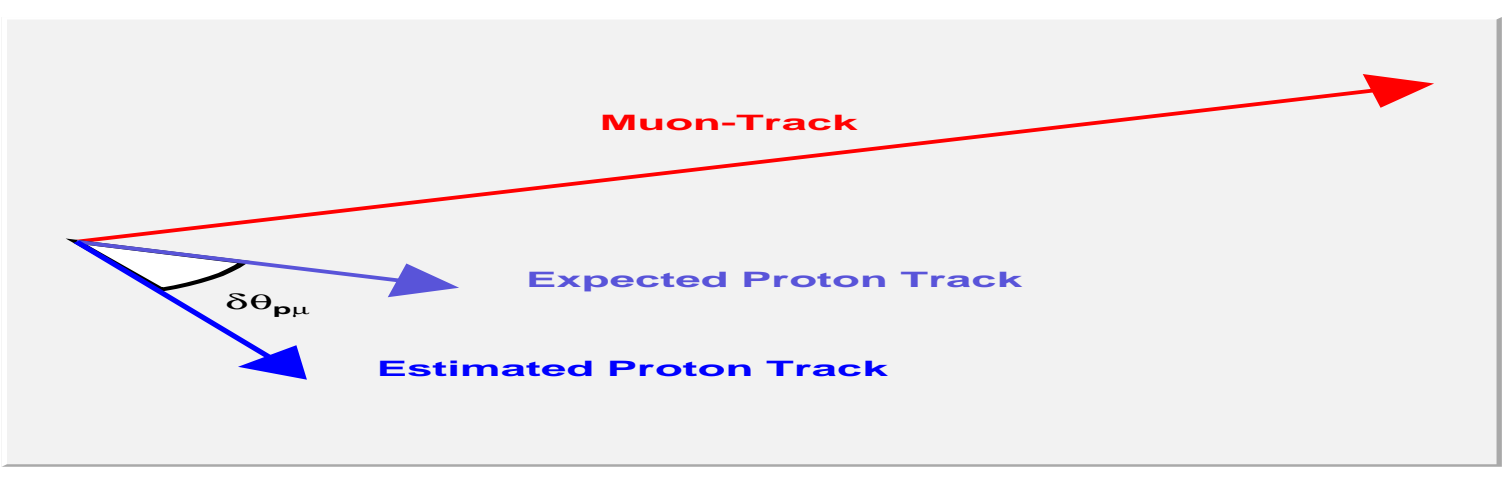

FIG. 29: Proton's angular difference. Schematic view of angular differences is shown in the top figure. Incident neutrino, outgoing muon and proton are shown in black, red and blue. The angles converted by QE and CCQE energy spectra are shown in light and dark blue. 
where $E_{\nu}, Q^{2}, E_{p}, p_{p}$ and $m_{p}$ are neutrino energy, momentum transfer, proton energy, momentum and mass. The detailed calculation is described in the Appendix C.

Step 3. - Taking angular differences : The opening angular formula depends on the neutrino energy. QE and CCQE energy formula can convert the angle into two different forms of $\theta^{Q E}$ and $\theta^{C C Q E}$. The angle calculations are constrained in physical region. Difference between QE and CCQE angles minimized, $\delta \theta=\left|\theta^{Q E}-\theta^{C C Q E}\right| \sim 0$ if selected events are $\mathrm{QE}$ events. Assume that $\delta \theta=0$ if the event has no shower, and $\delta \theta=\pi$ if those angles are in unphysical region. If the CCQE angle matches the QE predicted angle, within $3^{\circ}$, then it is likely to be a QE event. If the CCQE angle is more than $3^{\circ}$ from the $\mathrm{QE}$ predicted angle, then it is likely not a $\mathrm{QE}$ event, as shown in Figure 30.

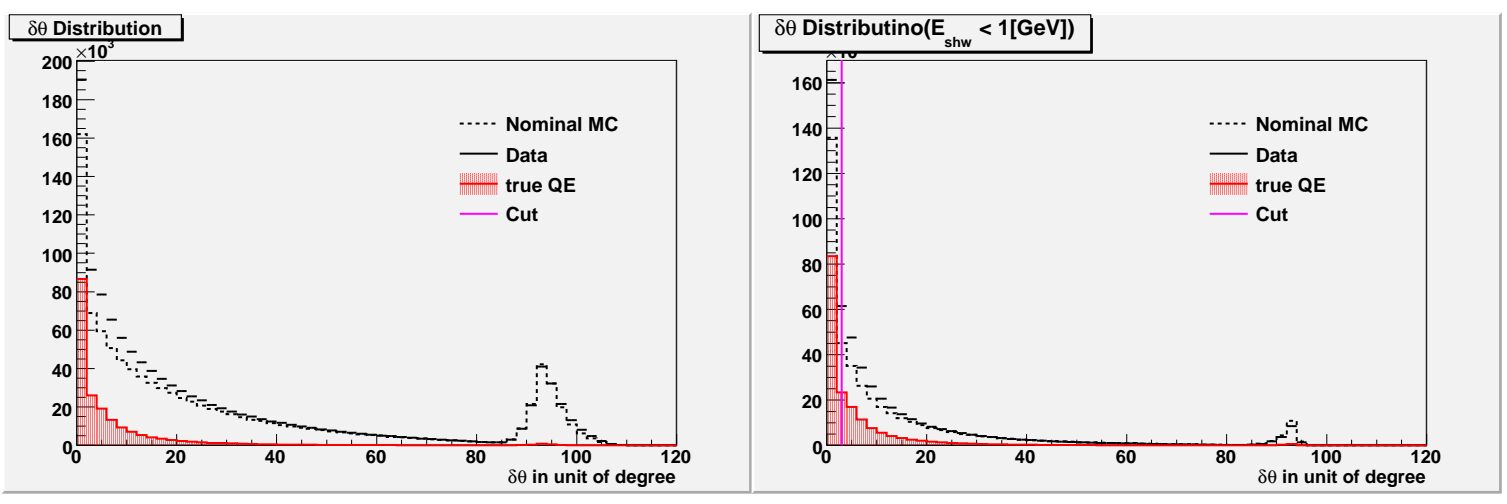

FIG. 30: Proton's angular difference. The angular difference between QE and CCQE spectra is used to select QEL-events. $\delta \theta$ distributions cuts are shown in the left and right figures. A shower energy cut is applied for the right figure. 


\section{Low- vs High- $Q^{2}$ Selection}

Energy transformation through the QEL interaction is not only sensitive to chang$\operatorname{ing} M_{A}^{Q E L}$ scale, but also it is linearly correlated to hadronic shower energy. It is written in the form of $Q^{2} \approx 2 m_{n} E_{\text {shw }}\left(x=Q^{2} / 2 m_{N} E_{\text {shw }}=1\right.$ if the QEL interaction). The relation allows us to consider two types of event selection methods : Low- and High- $Q^{2}$ selections.

If the interaction weren't sufficiently energetic, then the proton remains in nucleus and no hadronic shower can be produced in the detector. Thus low- $Q^{2}$ events can be selected by the low shower energy cut. The QEL interactions are dominant at low shower energy below $0.250 \mathrm{GeV}$. This cut is mainly used in first MINOS $M_{A}^{Q E L}$ Analysis [78]. Efficiency ${ }^{5}$ and purity $^{6}$ are $54.0 \%$ and $64.6 \%$ in total. Figure 31 shows that the purity and efficiency as a function of neutrino energy and momentum transfer. The purity is relatively flat as a function of $Q^{2}$, but the efficiency falls off at the higher $Q^{2}$ events. The purity and efficiency are conversely correlated as a function of $E_{\nu}$. The purity initially rises and the efficiency drops. The cross relation between the purity and the efficiency reflects the fact that at very low neutrino energies all interaction types are likely to leave only small amounts of shower energy whereas at 3-4 GeV the RES and DIS events will produce larger showers in the detector and not pass the QEL-like selection cut. The efficiency and purity are flat above $4 \mathrm{GeV}$. Figure 31 shows the flat data/MC difference in the $Q^{2}$ distribution.

\footnotetext{
${ }^{5}$ Efficiency is the probability that QEL events are survived when applying the QEL selection and written in the form of : Efficiency $=\frac{\# \text { of true QEL events after applying the } Q E L \text { selection }}{\# \text { of totaltrue QEL events with the initial cuts }}$.

${ }^{6}$ Purity is the probability that contains true QEL events in overall selected events and formed as :

Purity $=\frac{\# \text { of true } Q E L \text { events after applying the } Q E L \text { selection }}{\# \text { of the total events after applying the QEL selection }}$.
} 

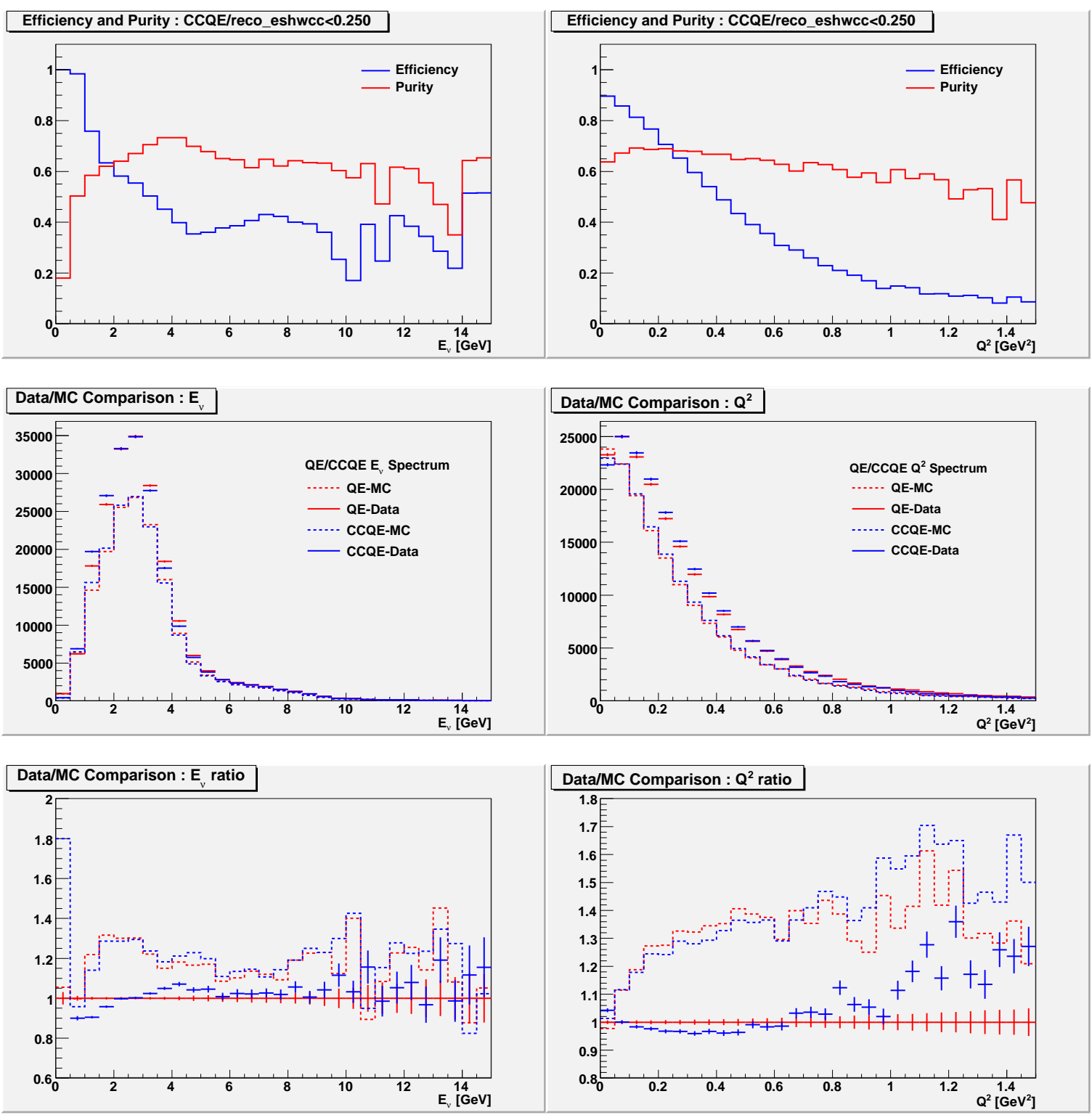

FIG. 31: The low- $Q^{2}$ selection is used for QEL event selection. Purity and efficiency are shown in the top two figures as a function of neutrino energy and momentum transfer. The $E^{\nu}$ and $Q^{2}$ distributions and data/MC ratios are shown in the middle and bottom two figures. QE and CCQE energy reconstruction are indicated in red and blue. 

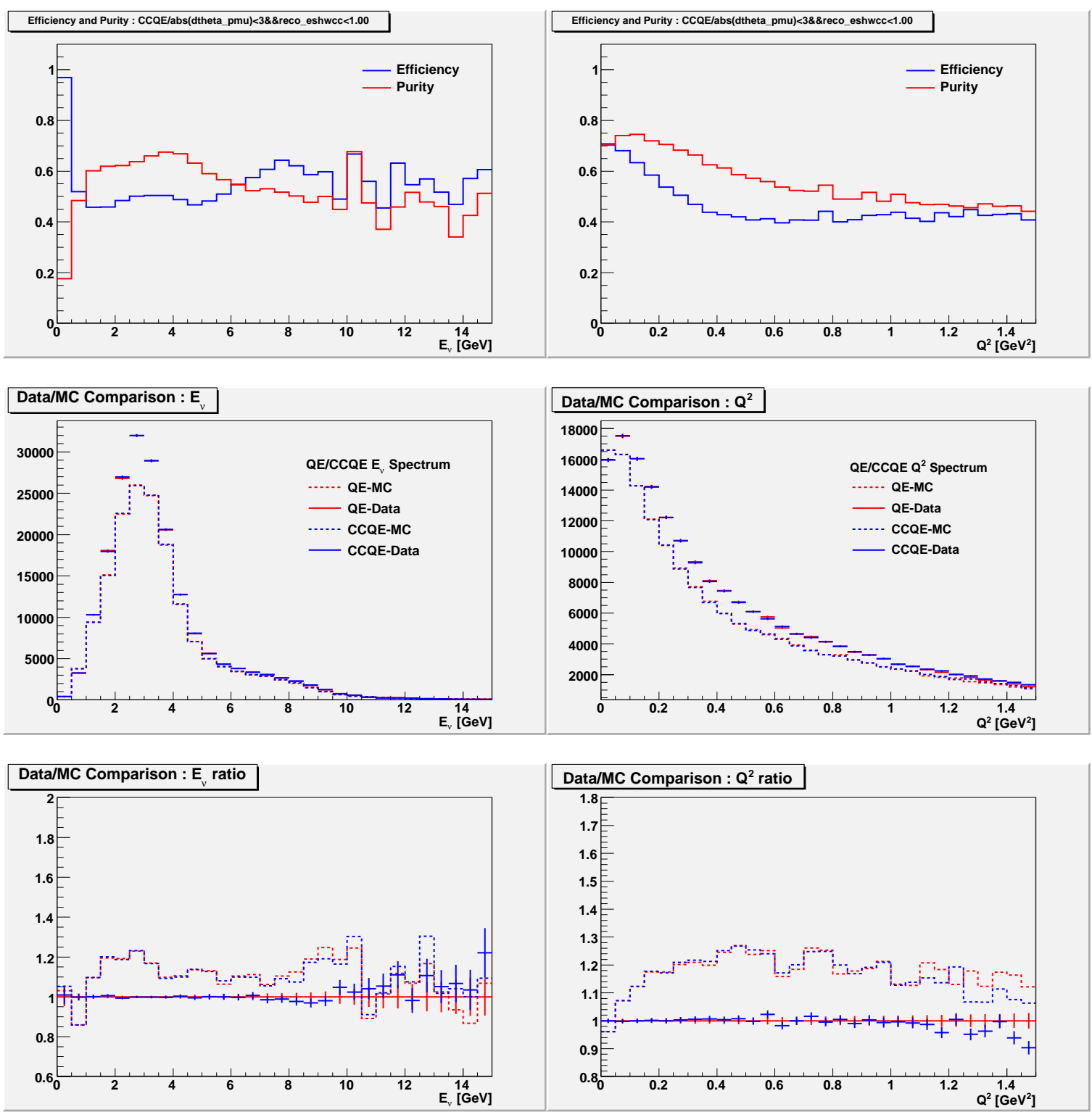

FIG. 32: The high- $Q^{2}$ selection is used for QEL event selection. Purity and efficiency are shown in the top two figures as a function of neutrino energy and momentum transfer. The $E^{\nu}$ and $Q^{2}$ distributions and data/MC ratios are shown in the middle and bottom two figures. QE and CCQE energy reconstruction are indicated in red and blue. 
On the other hand, if the interaction was energetic and generates enough energy for a proton to get out of the nucleus, then a few hundred $\mathrm{MeV}$ of shower energy can be produced in the detector. Thus high- $Q^{2}$ events are selected by high shower energy and the angle cut. Additional kinematic cut of the muon-proton opening angle shows that the QEL interactions are dominated at $\delta \theta$ below $3^{\circ}$. These cuts are described in previous section. Efficiency and purity are $49.5 \%$ and $62.4 \%$ in total. Figure 32 shows that the purity and efficiency as a function of $E_{\nu}$ and $Q^{2}$. Similarly the cross relation is observed between the purity and efficiency as a function of $E_{\nu}$. The purity is flat as a function of $Q^{2}$, but the efficiency is flat at higher $Q^{2}$ events. Figure 32 shows the flat data/MC difference in the $Q^{2}$ distribution.

\section{Event Distribution/Beam Stability}

Roughly $10^{7}$ CC-like events for $1.27 \times 10^{20}$ POT data set are observed in the Near Detector. These data provide high statistics data set to verify the performance of the Near Detector and the quality of CC- and QEL-like event selection procedure. $\delta \theta<3^{\circ}$ and $E_{s h w}<1 \mathrm{GeV}$ are applied for QEL-like event selection. Track vertex and angular distributions are shown in Figure 33. Track $r^{2}$ - and z-vertex distributions are expected to be flat over the detector. Black and red lines show CC- and QEL-like events. Flat vertex distributions are observed when applying the initial cut and the QEL-like event selection. The track angles are distributed pointing to the neutrino beam direction of $3^{\circ}$ downward to Near and Far detectors. CC- and QEL-like events are clearly peaked at the beam direction.

The beam energy spectrum measured during monthly period and the number of reconstructed events as a function of time are shown in Figure 34. The CC- and QEL-like beam energy distributions are consistent within statistical errors and show 

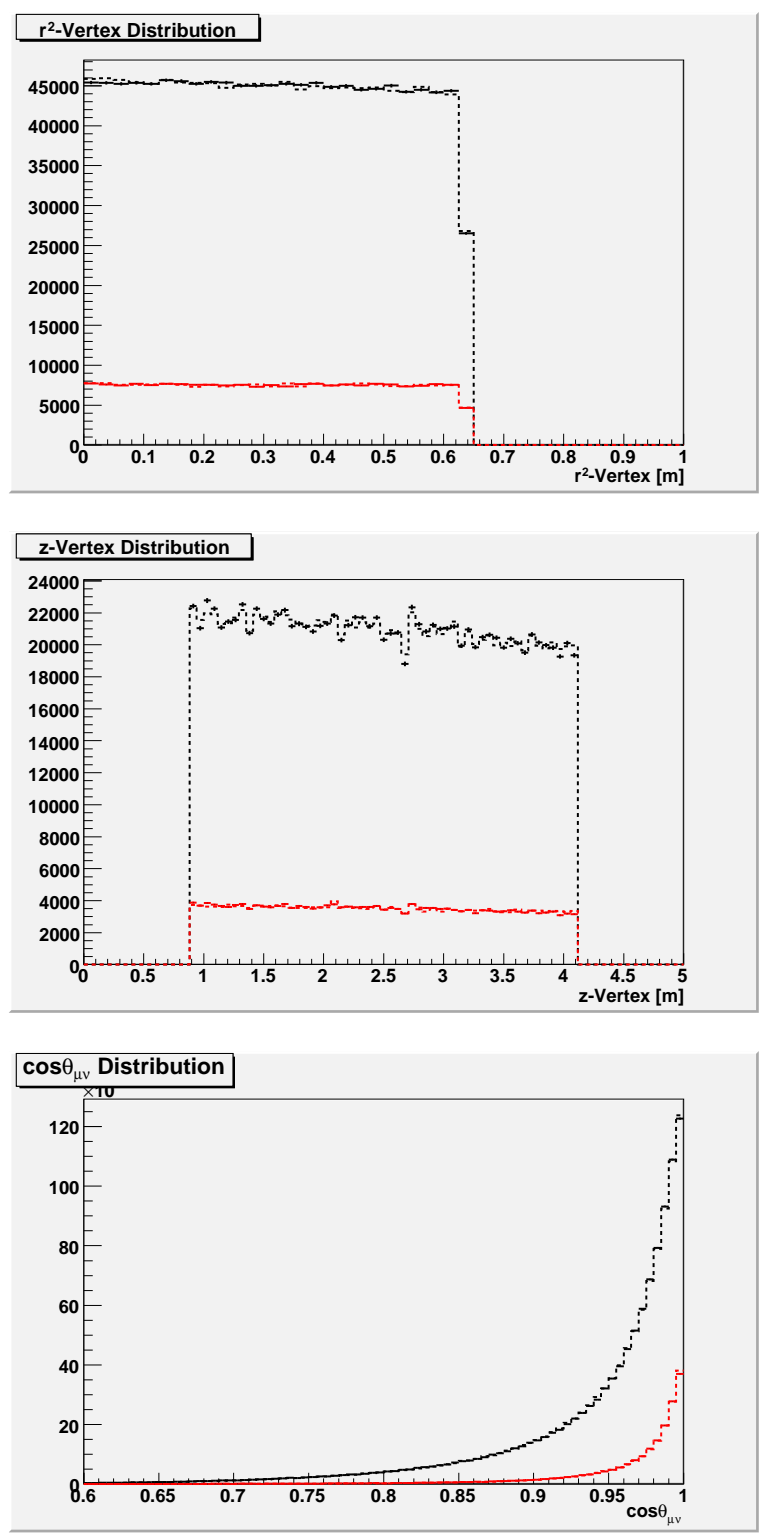

FIG. 33: Near Detector event distribution. $r^{2}$ - and $z$-vertex distributions are shown in top and middle figures. They are flat over the detector. The bottom figure shows the distributions of the track angles relative to the neutrino beam direction. 

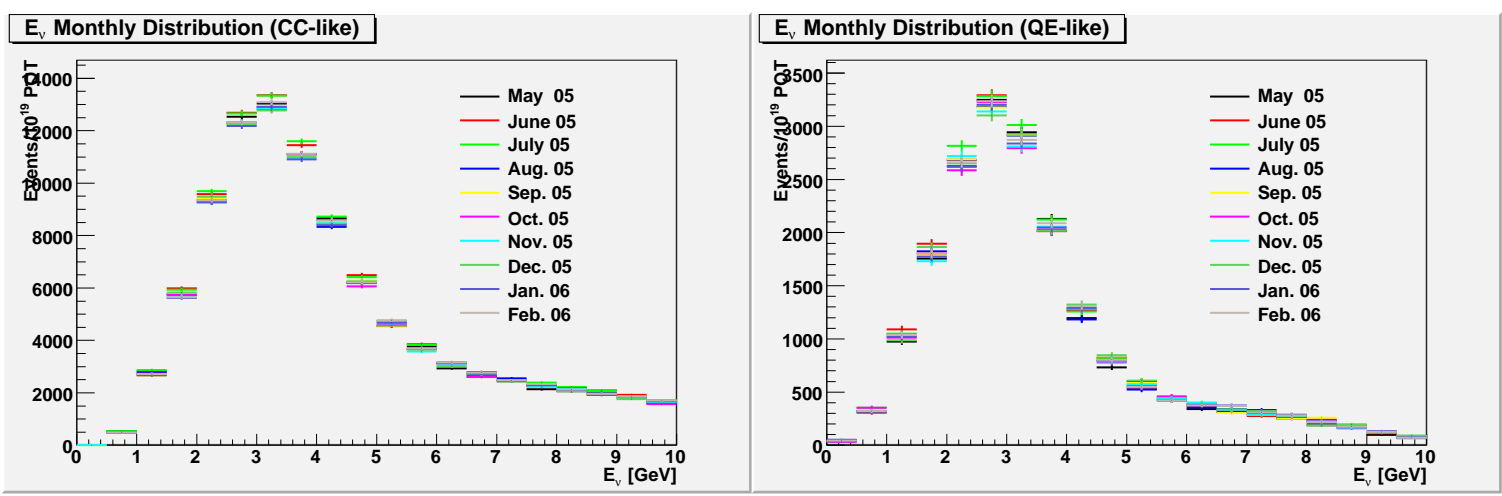

FIG. 34: Near Detector neutrino energy distribution taken during monthly period. The CC- and QEL-like event distributions measured during the monthly period are shown in left and right figures. The distributions are normalized to POT and only data obtained in the LE-10/185kA configuration is included.

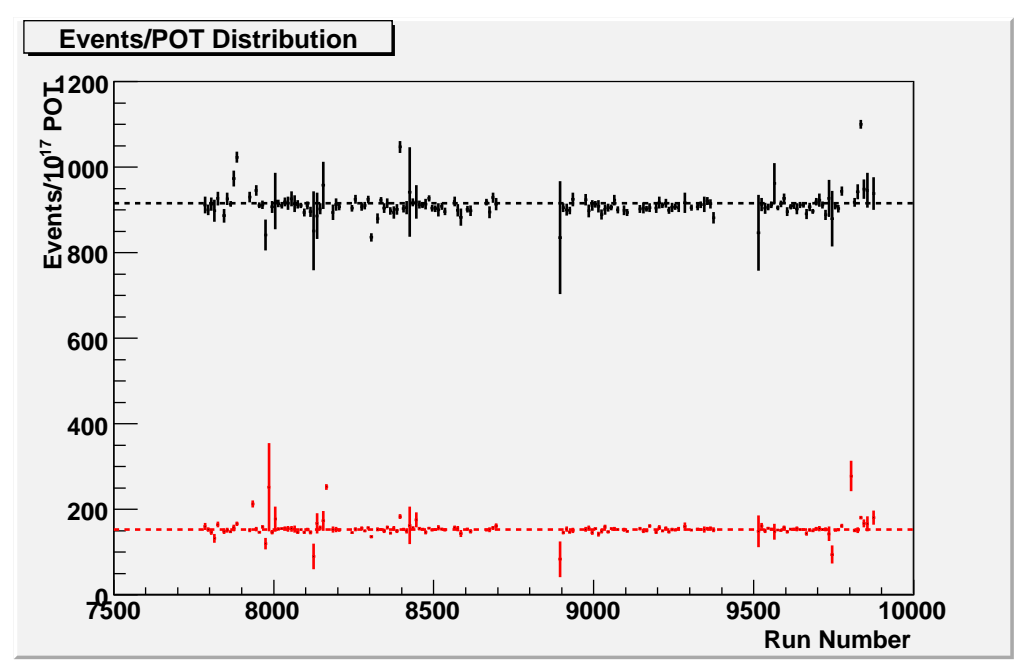

FIG. 35: Beam stability. The event number respect to the POT counting is shown as a function of time or run number. 
no rate dependence. The event numbers respect to POT counting are shown as a function of time or run number in Figure 35. Selected events are flat and stable over this data taking time period.

\section{Near Detector Fit}

Near Detector analysis is performed with Near Detector data set of $1.27 \times 10^{20}$ POT. The $E_{\nu}^{Q E}$ and $Q^{2}$ distributions are simultaneously fitted to include the QEL cross section parameter effects and to adjust the various systematic parameters in Near Detector MC spectra. These distributions are absolutely normalized by the POT counting.

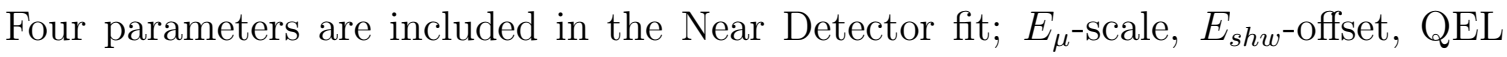
and RES $M_{A}$-scales. The $M_{A}^{Q E L}$ parameter is treated as free parameter. Systematic parameters are the $E_{\mu}$-scale, $E_{s h w}$-offset, QEL and RES $M_{A^{-}}$-scales. Single pion resonance production is one of the dominant background processes and formulated with the $M_{A}^{R E S}$ parameter in generator cross section model. Changing the $M_{A}^{R E S}$ scale largely influences the shape and normalization of the $Q^{2}$ distribution. Additionally, $Q^{2}>0.2 \mathrm{GeV}^{2}$ is applied to avoid the nuclear effects. The general principles of the fit are described below.

$\chi^{2}$ Definition : The statistical minimization function in the fit is defined as follows:

$$
\chi^{2}\left(\alpha_{0}, \ldots \alpha_{n s y s t}\right)=\sum_{i=0}^{n b i n s} \frac{\left(N_{i}^{e x p}-N_{i}^{\text {obs }}\right)^{2}}{\sigma_{\text {exp }, i}^{2}+\sigma_{o b s, i}^{2}}+\sum_{j=0}^{n s y s t} \frac{\Delta \alpha_{j}^{2}}{\sigma_{\alpha_{j}}^{2}}
$$

where $N_{i}^{e x p}=N_{i}^{e x p}\left(\alpha_{0}, \ldots . \alpha_{n s y s t}\right)$ and $N_{i}^{\text {obs }}$ are the numbers of expected MC events and observed data events. $\alpha_{j}$ are the fitted systematic parameters, with associated errors $\sigma_{\alpha}$. The second term penalizes the value of $\chi^{2}$ as the systematic parameters are varied away from their nominal values. The fit minimization is performed using 
the MIGRAD algorithm provided by TMinuit routine in ROOT software package. (The ROOT package is available at http ://root.cern.ch.) It is a variable-metric method with inexact line search, a stable metric updating scheme, and checks for positive-definiteness. Its disadvantage is that it depends heavily on knowledge of the first derivatives, and fails miserably if they are very inaccurate. It is not the best minimization algorithm, but it is the one used by the MINOS collaboration.

Binning : The binning is chosen as follows:

- 21 bins with $0.5 \mathrm{GeV}$ bin width in the range of $E_{\nu}^{Q E} \in[0: 10] \mathrm{GeV}$. Overflow bin is above $10.0 \mathrm{GeV}$.

- 21 bins with $0.05 \mathrm{GeV}^{2}$ bin width in the range of $Q^{2} \in[0: 10] \mathrm{GeV}^{2}$. Overflow bin is above $1.0 \mathrm{GeV}^{2}$.

Fit Result : The four parameter fit result is shown in Figure 36. The best fit value is $M_{A}^{Q E L}=1.172 \mathrm{GeV}$ and doesn't agree well to $\chi^{2}=228.890 / 37$. The best fit values of the other systematic parameters are 1.000 in the $E_{\mu}$ scale, $-0.0517 \mathrm{GeV}$ in $E_{\text {shw }}$ offset and 0.944 in the $M_{A}^{R E S}$ scale. More fit results are described in Appendix D. Thus, the Near Detector data is not well explained adjusting the QEL cross section parameter and assuming a point-like shower variable. However, the magnitude of the discrepancies between the data and best fit values depends on the overall normalization. $5-20 \%$ data/MC ratio is observed in the low energy region while the fit well agrees in the high energy region. Initial excursion in the data/MC ratio of $\sim 30 \%$ is reduced to $\sim 10 \%$. 

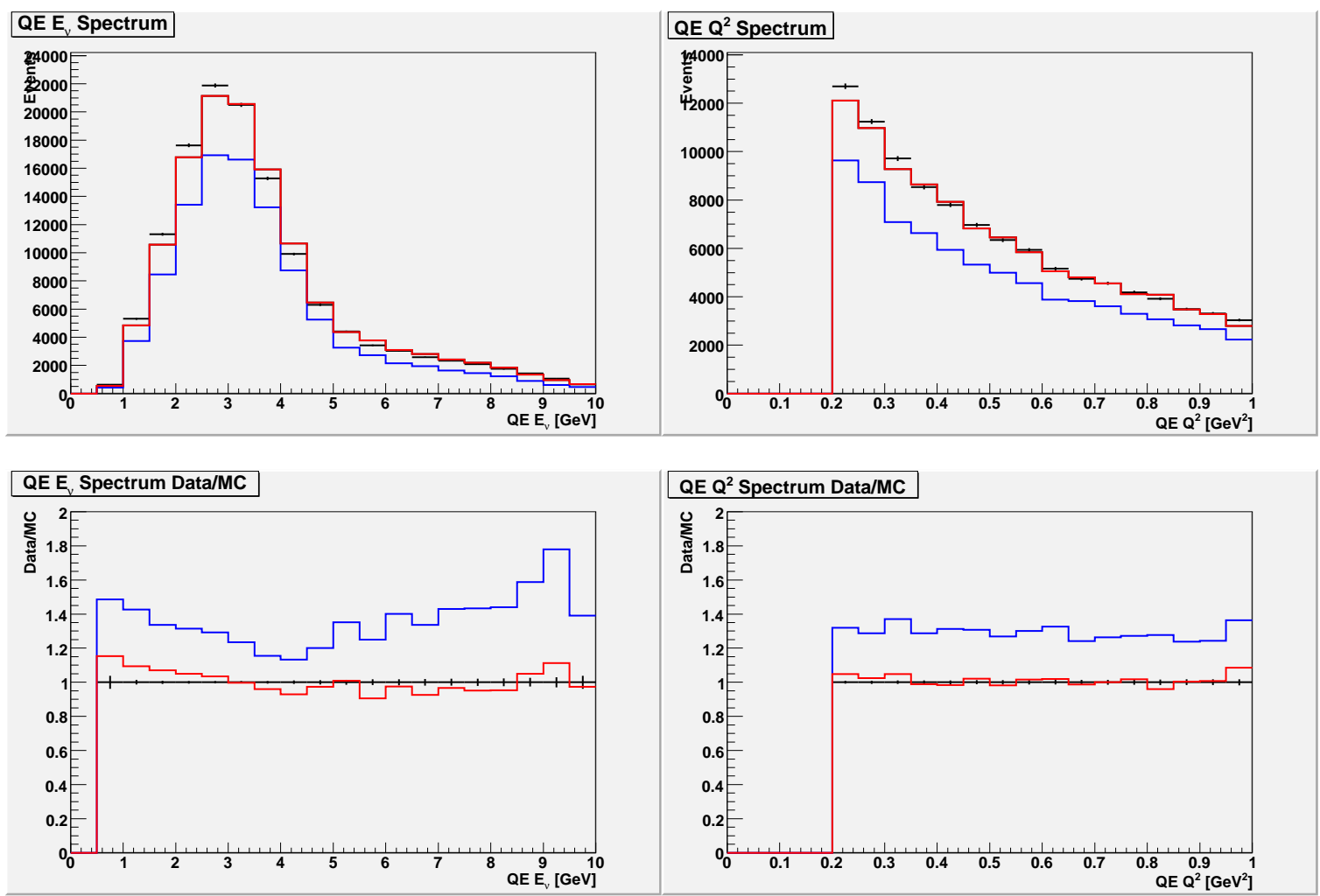

FIG. 36: $E_{\nu}$ and $Q^{2}$ distributions of the Near Detector fit to the data. Blue and red lines are for the nominal and best fit spectra. 
Systematic Uncertainties : Systematic uncertainties from various sources are described in detail below.

(1) Beam Production Model : The neutrinos from the NuMI beam are produced from the decays of secondary pions and kaons produced in the NuMI target. The pions account for the majority of the low neutrino flux $\left(E_{\nu}<30 \mathrm{GeV}\right)$ while the kaons are dominant at the higher energies. The $E_{\nu}^{Q E}$ and $Q^{2}$ distributions are weighted by the best fit of the SKZP beam parameterization. The SKZP parameter fit is organized with 26 parameters in total. (i) 16 parameters are used for the hadron productions. The yield and differential yield of the secondary hadron productions are the dominant uncertainties in predicting the neutrino flux from the beamline. The differential yields from the NuMI target are formulated in

$$
\frac{d^{2} N}{d x_{F} d p_{T}}=\left[A+B p_{T}\right] e^{-C p_{T}^{3 / 2}}
$$

where $\mathrm{A}, \mathrm{B}$ and $\mathrm{C}$ are parameterized as a function of $x_{F}$. The $p_{T}$ distributions of negative pions and kaons from the NuMI target are calculated using Fluka2005, for several bins of $x_{F}$. They are defined as follows :

$$
\begin{aligned}
& A\left(x_{F}\right)=a_{1}\left(1-x_{F}\right)^{a_{2}}\left(1+a_{3} x_{F}\right) x_{x_{F}}^{-a_{4}} \\
& B\left(x_{F}\right)=b_{1}\left(1-x_{F}\right)^{b_{2}}\left(1+b_{3} x_{F}\right) x_{x_{F}}^{-b_{4}} \\
& C\left(x_{F}\right)= \begin{cases}c_{1} / x_{F}^{c_{2}}+c_{3} & \text { if } x_{F}<0.22 \\
c_{1} e^{c_{2} x_{F}-c_{3}}+c_{4} x_{F}+c_{5} & \text { if } x_{F}>0.22\end{cases}
\end{aligned}
$$

where $x_{F}=p_{z} / 120 \mathrm{GeV}$ and $a_{i}, b_{i}$ and $c_{i}$ are parameter coefficients. The importance weights for each meson are determined with 16 hadron production parameters ( $p_{0}$ to $\left.p_{15}\right)$ in Equation (4.10) to (4.13). The ratios of target yield $\pi^{-} / \pi^{+}$and $K^{-} / K^{+}$ are well constrained with $5 \%$ and $20 \%$ uncertainties at $1 \sigma$ level. The weights for negatively charged mesons are linearly correlated to the positively charged ones and 
determined by a simultaneous fit to anti-muon neutrino data samples.

$$
\begin{aligned}
W\left(\pi^{+}, x_{F}\right) & =\frac{\left[A_{\pi^{+}}^{\prime}+B_{\pi^{+}}^{\prime} p_{T}\right] e^{-C_{\pi^{+}}^{\prime} p_{T}^{3 / 2}}}{\left[A_{\pi^{+}}+B_{\pi^{+}} p_{T}\right] e^{-C_{\pi^{+}} p_{T}^{3 / 2}}} \\
W\left(K^{+}, x_{F}\right) & =\frac{\left[A_{K^{+}}^{\prime}+B_{K^{+}}^{\prime} p_{T}\right] e^{-C_{K^{+}}^{\prime} p_{T}^{3 / 2}}}{\left[A_{K^{+}}+B_{K^{+}} p_{T}\right] e^{-C_{K^{+}} p_{T}^{3 / 2}}} \\
W\left(\pi^{-}, x_{F}\right) & =\left(p_{12}+p_{13} x_{F}\right) W\left(\pi^{+}, x_{F}\right) \\
W\left(K^{-}, x_{F}\right) & =\left(p_{14}+p_{15} x_{F}\right) W\left(K^{+}, x_{F}\right)
\end{aligned}
$$

where $A\left(x_{F}\right), B\left(x_{F}\right)$ and $C\left(x_{F}\right)$ are redefined in following;

$$
\begin{array}{ll}
A_{\pi^{+}}^{\prime}\left(x_{F}\right)=\left(p_{0}+p_{1} x_{F}\right) A_{\pi^{+}}\left(x_{F}\right) & A_{K^{+}}^{\prime}\left(x_{F}\right)=\left(p_{6}+p_{7} x_{F}\right) A_{K^{+}}\left(x_{F}\right) \\
B_{\pi^{+}}^{\prime}\left(x_{F}\right)=\left(p_{2}+p_{3} x_{F}\right) B_{\pi^{+}}\left(x_{F}\right) & B_{K^{+}}^{\prime}\left(x_{F}\right)=\left(p_{8}+p_{9} x_{F}\right) B_{K^{+}}\left(x_{F}\right) \\
C_{\pi^{+}}^{\prime}\left(x_{F}\right)=\left(p_{4}+p_{5} x_{F}\right) C_{\pi^{+}}\left(x_{F}\right) & C_{K^{+}}^{\prime}\left(x_{F}\right)=\left(p_{10}+p_{11} x_{F}\right) C_{K^{+}}\left(x_{F}\right)
\end{array}
$$

(ii) 5 parameters are related to the systematic uncertainties of the target position; horn 1 offset $(1 \sigma=1 \mathrm{~mm})$, baffle scraping $(1 \sigma=0.25 \%)$, POT uncertainty $(1 \sigma=$ $2 \%)$, and horn current miscalibration $(1 \sigma=1 \%)$ and distribution $(1 \sigma=$ difference between $\delta=$ inf and $\delta=6 \mathrm{~mm}$ ). (iii) The other 5 parameters are used for taking into account the detector and $\overline{\nu_{\mu}}$ cross section effects; neutrino energy miscalibration $(1 \sigma=5 \%)$, shower energy offset $(1 \sigma=50 \mathrm{MeV}), \mathrm{NC}$ for $\nu_{\mu}$ and $\overline{\nu_{\mu}}(1 \sigma=30 \%)$, and $\overline{\nu_{\mu}}$ cross section $(1 \sigma=30 \%)$. More details are well studied in Ref. [73].

(2) Muon Energy Scale : Calculation of $E_{\nu}^{Q E L}$ and $Q^{2}$ strongly depends on muon momentum for each event. Changing $E_{\mu}$-scale will affect the shape of $Q^{2}$ distributions and $M_{A}^{Q E L}$ measurement. Taking into account of the uncertainties in $\mathrm{dE} / \mathrm{dX}$ for both range and curvature measurement yields a $2 \%$ uncertainty of absolute muon energy 
scale. A $2 \%$ change in muon energy scale gives $\pm 0.08 \mathrm{GeV}$ error in the $M_{A}^{Q E L}$ fit value. Approximately shifting $\sim \pm 0.05 \mathrm{GeV}$ in $M_{A}$-scale can propagate to change a small number of events up or down in one $E_{\nu}$ and $Q^{2}$ bins. Figure 37 shows that changing the muon energy scale largely influences the shape of the $Q^{2}$ and $E_{\nu}$ distributions.
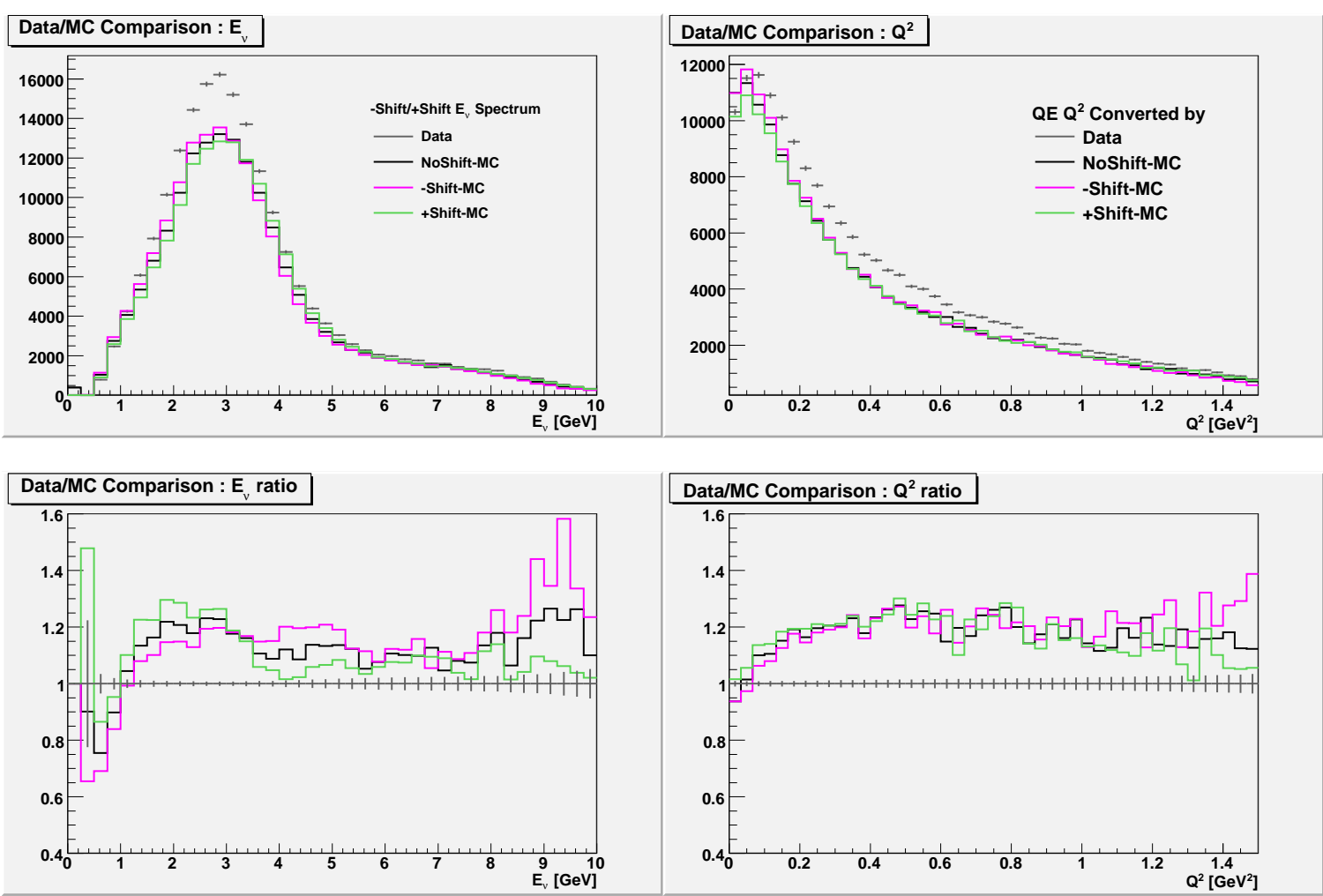

FIG. 37: \pm 0.02 shifts of $E_{\mu}$-scale for the $E_{\nu}$ and $Q^{2}$ distributions and data/MC ratios.

(3) Absolute Shower Energy Scale : $10 \%$ and $0.050 \mathrm{GeV}$ uncertainties of absolute shower energy scale and offset are the largest systematic effect, which are the sum in quadrature of a $5.7 \%$ uncertainty in the calorimeter response to hadrons as determined from the test beam measurements, a $2.3 \%$ uncertainty in the energy scale calibration and a $8.2 \%$ uncertainty due to the intranuclear rescattering effect of miss- 
ing pion energy in the final state of hadrons [44, 76, 77]. Figures 38 and 39 show that shower energy scale and offset shifts in the $Q^{2}$ and $E_{\nu}$ distributions. Normalization of the $Q^{2}$ and $E_{\nu}$ distributions is mainly influenced by changing the shower energy scale. On the other hand, the shape of the $Q^{2}$ distribution is largely affected by changing the offset.
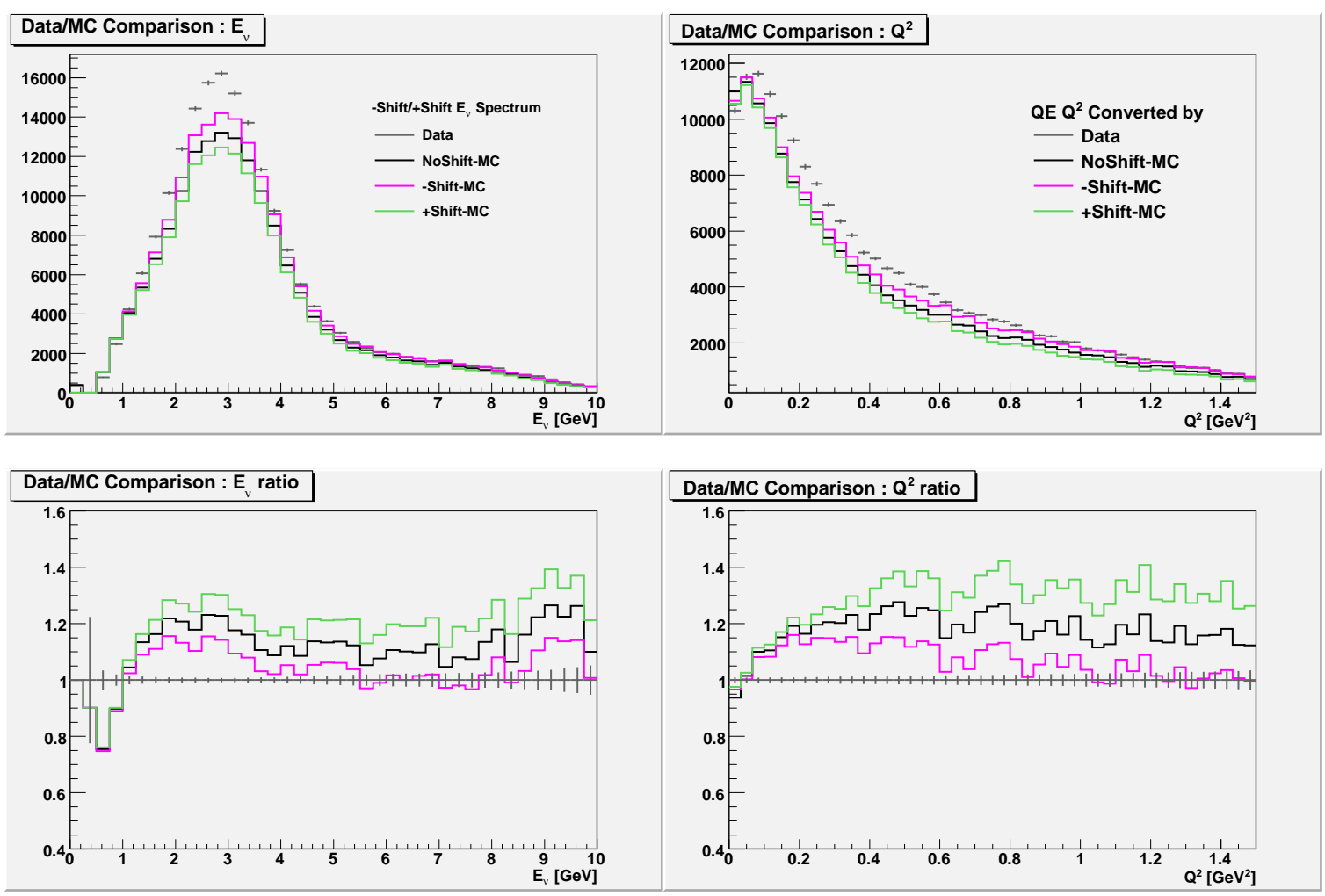

FIG. 38: \pm 0.10 shifts of $E_{s h w}$-scale for the $E_{\nu}$ and $Q^{2}$ distributions and data/MC ratios. 

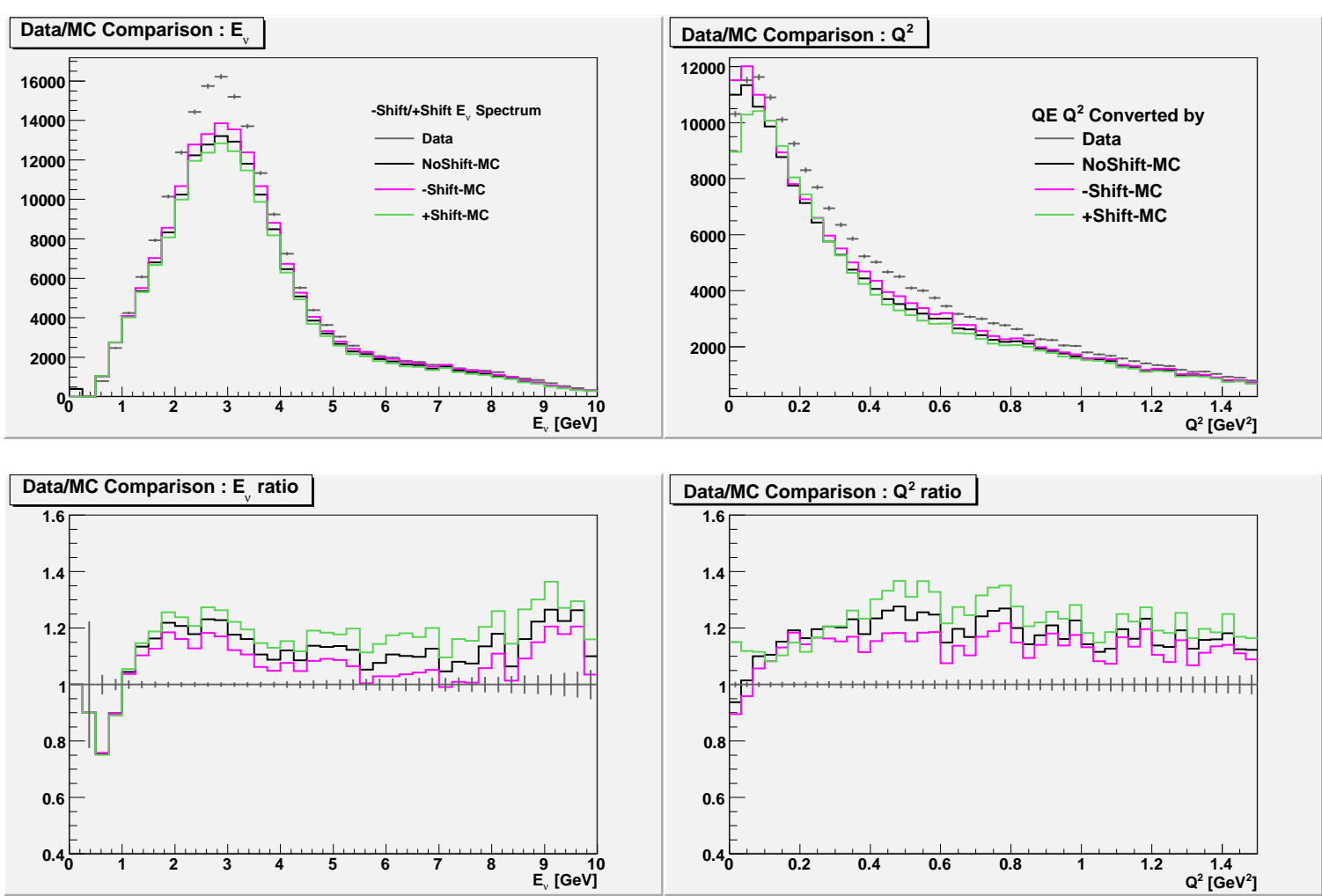

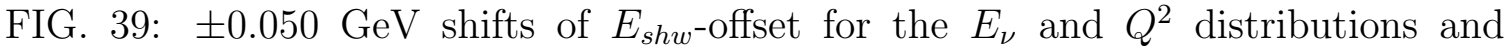
data/MC ratios.

(4) Neutrino-Nucleon Interactions : Neutrino-nucleus interactions in the MINOS detectors are modelled using the NEUGEN event generator [79]. NEUGEN simulates the quasi elastic and inelastic neutrino scattering processes that occur in the MINOS detectors. Figure 21 shows the muon neutrino cross section per neutrino energy as a function of neutrino energy. Total CC cross section uncertainty can be broken down into systematic uncertainties in the normalization of the QEL, RES and RES/DIS-transition cross sections. These uncertainties are discussed below. 
(4.1) The inelastic background scale parameter is applied to non-QEL events in the QEL event sample, which are mainly composed of RES and DIS events. Contribution of the DIS events are small, but not negligible. A 5\% uncertainty for the QEL+RES cross section parameter and the $3 \%$ uncertainty for the normalization of the DIS cross section at high invariant mass $(W>1.7 \mathrm{GeV})$ are estimated from earlier global $Q^{2}$ and cross section fits of the deuterium experiments [72, 80, 79]. The DIS components of the QEL event sample are small and located at low invariant mass. Thus, a $1 \sigma$ uncertainty of $5 \%$ is assigned to the NonQE background scale parameter.
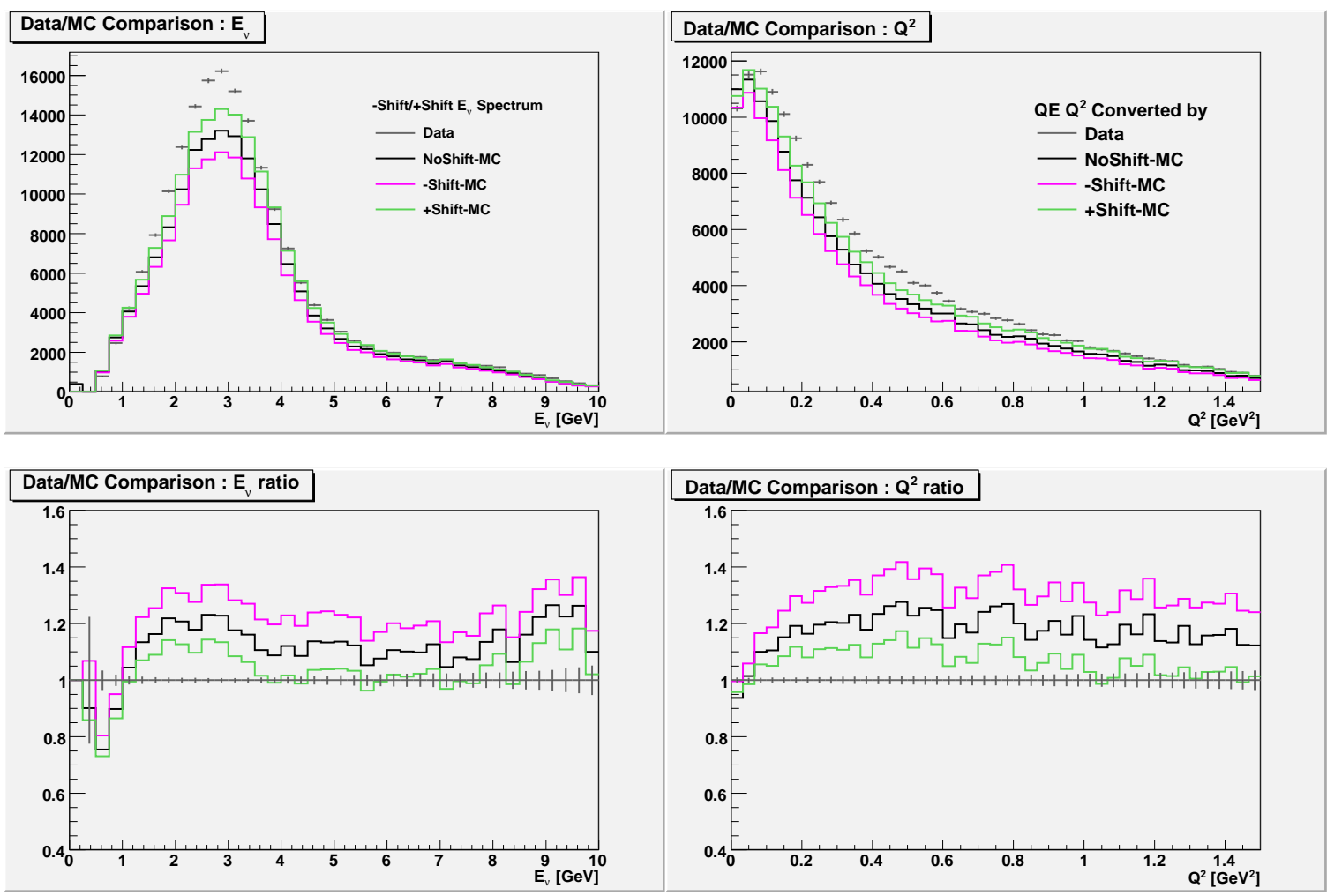

FIG. 40: \pm 0.15 shifts of $M_{A}^{Q E L}$-scale for the $E_{\nu}$ and $Q^{2}$ distributions and data/MC ratios. 
(4.2) The 5-15\% uncertainty of the QEL and RES cross sections also contributes to the source of systematic error. The QEL and RES cross sections are well modeled using two parameters in the dipole form of the axial-vector form factor. Nominal values of the QEL and RES $M_{A}$ are 0.99 and $1.12 \mathrm{GeV}$ respectively in the NEUGEN model. The primary effects of varying the QEL and RES $M_{A}$ parameters significantly appear to disturb the shape and normalization of the $Q^{2}$ distribution. Figures 40 and 41 show the effects of changing the QEL and RES $M_{A}$ scale in the $E_{\nu}$ and $Q^{2}$ distributions respectively and illustrates a change in the shape and normalization.
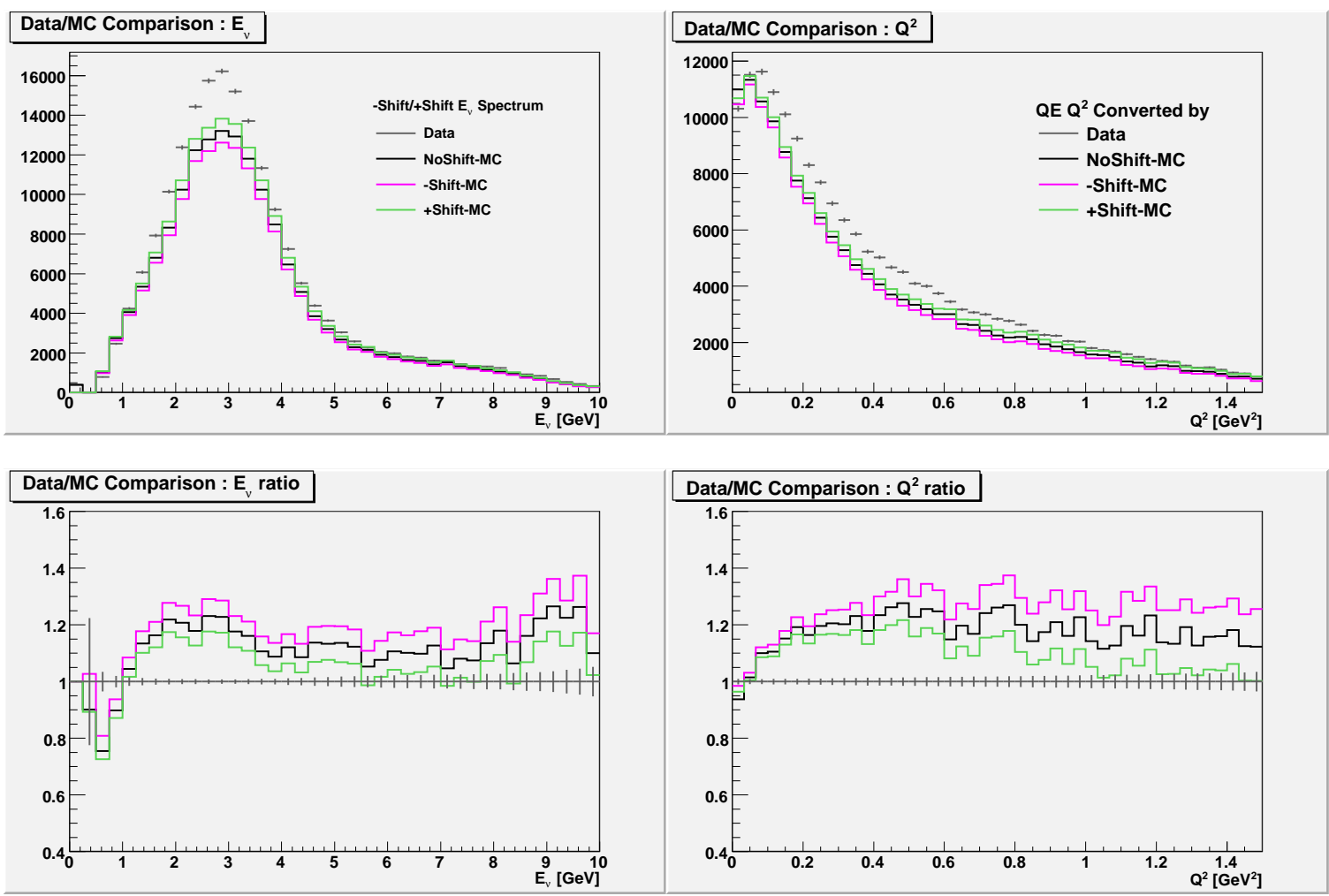

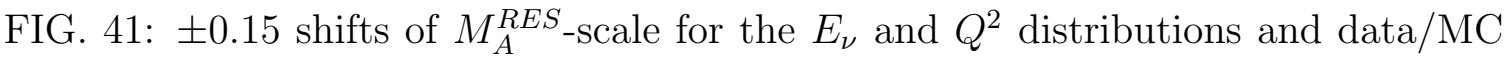
ratios. 
(4.3) The $10-20 \%$ uncertainty in the RES/DIS transition energy factors also contributes to the source of systematic error. NEUGEN uses a Rein-Sehgal based treatment of the RES production, and the $\mathrm{CC}$ and $\mathrm{NC}$ coherent pion productions and a modified DIS model extended to improve the treatment in the transition region between the DIS and RES production [79]. The cross section in the transition region is well modeled using the $r_{i j k}$ parameter in the NEUGEN model, where $i, j$ and $k$ indicate the $\mathrm{CC} / \mathrm{NC}$ interaction, initial state of interaction and pion multiplicity in final state of interaction. Estimated errors on the $r_{i j 2}$ and $r_{i j 3}$ parameters are 0.1 and 0.2 respectively. $\mathrm{a}+/-1 \sigma$ is defined to be simultaneous positive/negative shift of
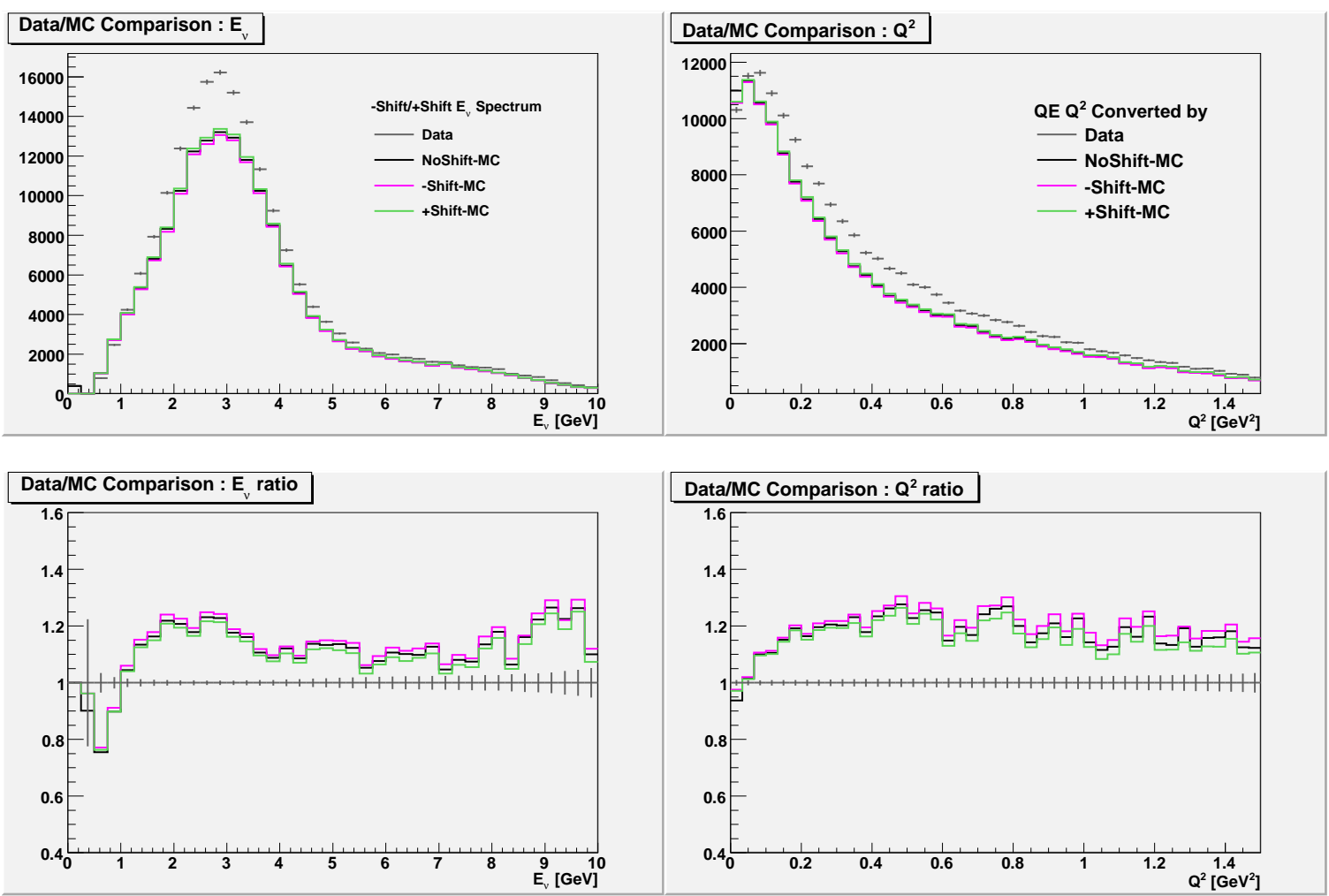

FIG. 42: $\pm 1 \sigma$ shifts of $M_{A}^{R E S}$-scale and RES/DIS-transition scale for the $Q^{2}$ and $E_{\nu}$ distributions and data/MC ratios. 
the estimated errors in all transition parameters. Figure 42 shows $\pm 1 \sigma$ shift of the transition scale in the $E_{\nu}$ and $Q^{2}$ distributions.

(5) The RFG model : Nuclear effects of the RFG model are dominant in the low- $Q^{2}$ region. In the RFG model, if all energy states are filled below the surface, then any interaction with the momentum transfer that leaves the final state nucleon with momentum less than the Fermi momentum is blocked by so called Pauli blocking. Figure 43 shows an example of the Pauli suppression in the low- $Q^{2}$ region, compared to a free nucleon. The $\kappa$ parameter in the RFG model can be adjusted to modify the level of the suppression. Alternatively, the $Q^{2}>0.2 \mathrm{GeV}^{2}$ cut is applied to avoid the nuclear effect in this analysis.

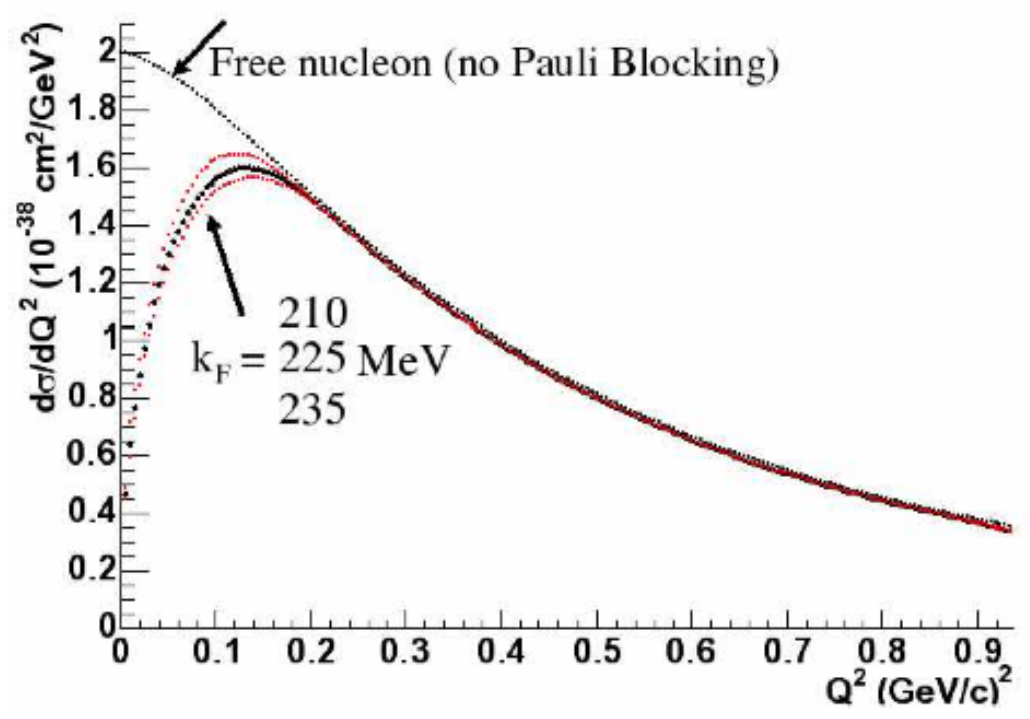

FIG. 43: Pauli blocking suppression effects. Differential neutrino-nucleon cross section is shown as a function of $Q^{2}$. Low- $Q^{2}$ values are largely influenced by the PauliSuppression effects in the RFG mode, compared to the free nucleon prediction. The figure is referenced from [78]. 


\section{The Far Detector Analysis}

The neutrino oscillation analysis is performed using Far Detector data. The unoscillated spectrum at the Far Detector is predicted using two different approaches. The first method uses the beam matrix method that directly converts the Near Detector data spectrum to the Far Detector. Relative adjustments of energy spectrum are made between the Near and Far Detectors and are less sensitive to uncertainties in the absolute flux, cross-sections and detector acceptance. The Near Detector data are used as measured without further constraining the MC simulation. The QEL oscillation analysis is studied using this method in Ref. [81]. The second method uses the Near Detector fit method that indirectly extrapolates the beam spectra using the Near Detector data to constrain the MC calculation of the neutrino flux, crosssections and detector acceptance [45]. The improved MC is then used to calculate the energy spectrum expected at the Far Detector. The QEL oscillation analysis performed here uses the Near Detector fit result, where the best values of the Near Detector systematic parameter fit are simply transfered to the Far Detector MC to predict the unoscillated spectrum.

\section{QEL Selection : QEL Kinematic Correction}

The Far Detector data set of $2.50 \times 10^{20}$ POT (cedar_phy version) taken during the period May 20, 2005 to Feb. 25, 2006 and Sep. 22, 2006 to March 31, 2007 is used for this oscillation analysis. The cumulative distribution of the number of total POT is shown as a function of time or run number in Figure 44. POT counting is proportional to run time. The low energy beam was not operational during the flat region between run number 9874 and 10776. The Far Detector data is initially

selected using the CC-like event cuts. CC events are selected with $P I D_{A B}>0.85$ 


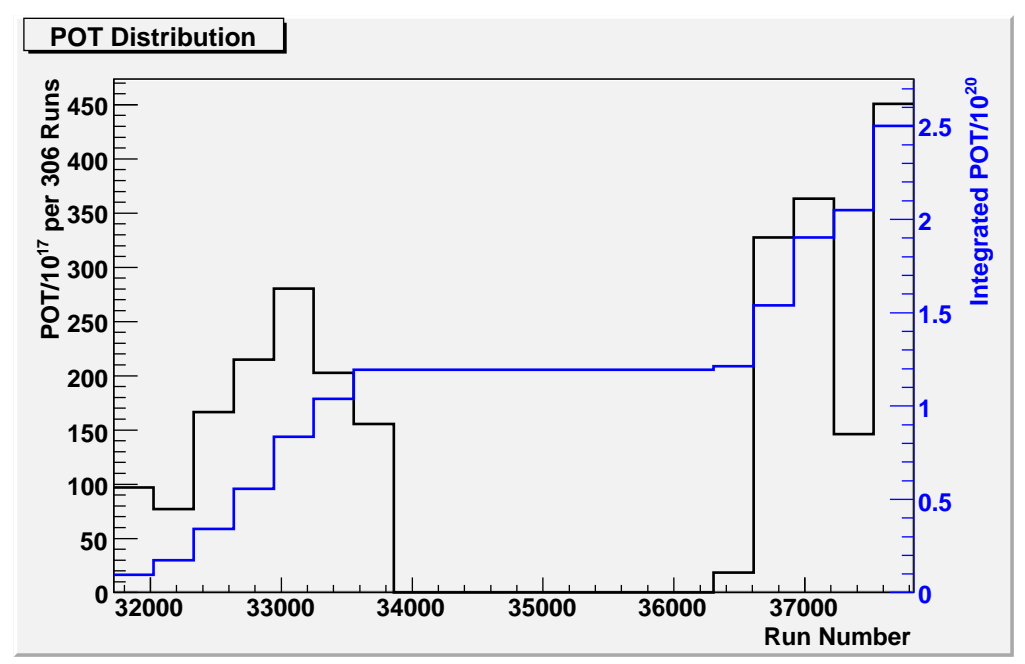

FIG. 44: POT and integrated POT distributions. POT and integrated POT distributions are shown as a function of time or run number. Low energy beam was not operation during the flat period.

[75]. The PID distribution is shown in Figure 45 (left). From the PID selection, the efficiency and purity are 0.911 and 0.991 respectively. They are shown as a function of neutrino energy in Figure 45 (right). MC/data sets and initial CC-like event selection cuts are listed as follows;

\section{Initial cuts :}

- 2008 Standard CC fiducial cut

- Negative signed one track event $($ ntrack $=1$ and $q / p<0$ )

- Track angle cut $\left(\cos \theta_{\mu \nu}>0.6\right)$

- CC-like cut : $P I D_{A B}>0.85$ 
Using the High- $Q^{2}$ selection described in the Near Detector analysis section, the proton-muon opening angular distribution is calculated for the Far Detector. Similarly $\delta \theta<3^{\circ}$ and $E_{\text {shw }}<1 \mathrm{GeV}$ are applied for QEL-like event selection for the Far Detector data. Shower energy and $\delta \theta$ distributions are shown in Figure 47. MC is normalized to the data event number. The purity and efficiency are 0.489 and 0.601 respectively for selecting QEL-like events. Figure 47 shows the purity and efficiency as a function of neutrino energy.
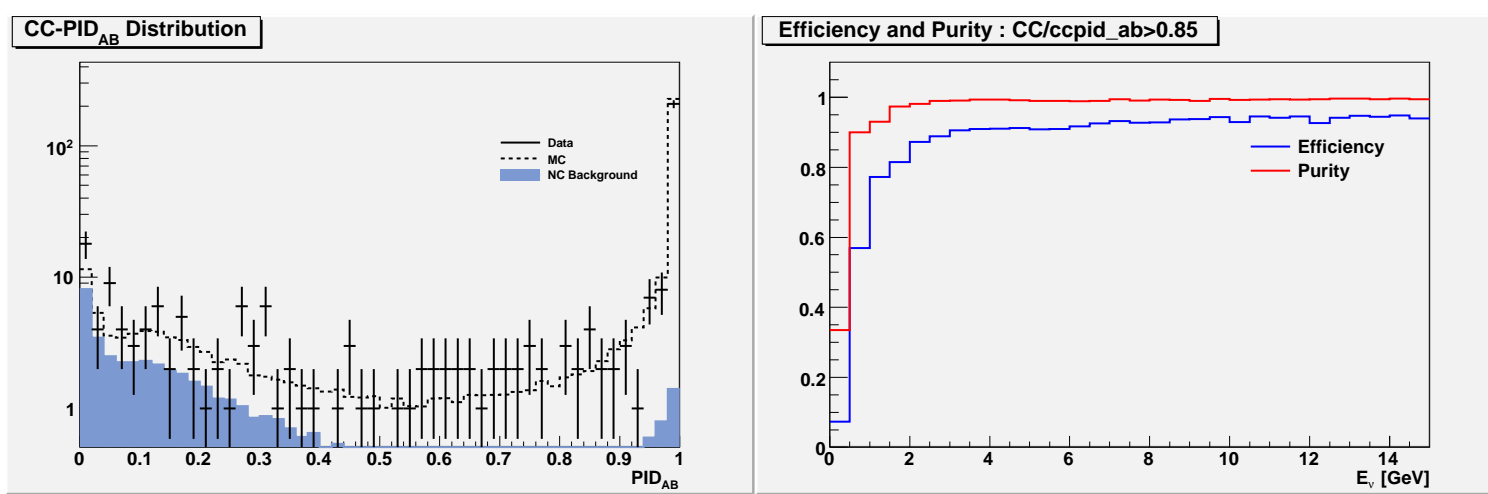

FIG. 45: Far Detector CC-like event distributions. (Left) PID(particle identification) is the parameter used to discriminate between CC- and NC-like events. The MC data is normalized to the data event number. (Right) Efficiency and purity of the CC-like event selection are shown in blue and red lines respectively. 

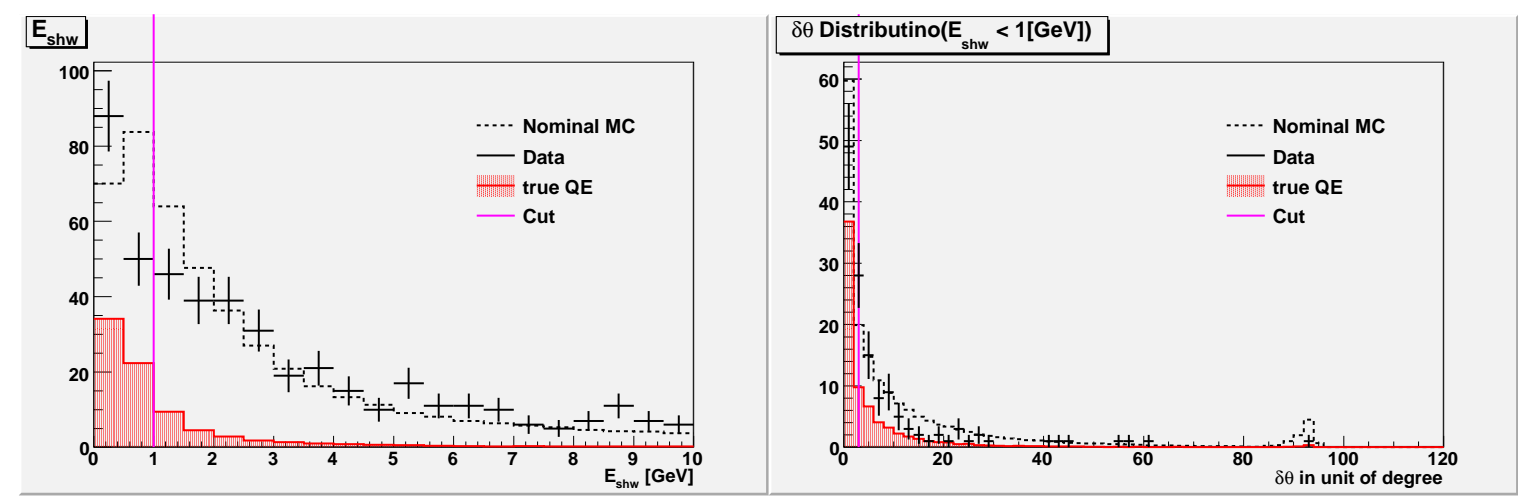

FIG. 46: Far Detector QEL-like event cuts. (Left) Shower energy distribution is shown with only initial cuts. The pink line is for the shower energy cut at $1 \mathrm{GeV}$. (Right) $\delta \theta$ distribution with the shower energy cut. The pink line is for the angle cut at $3^{\circ}$. MC is normalized to the data event number for the shower and angle figures.

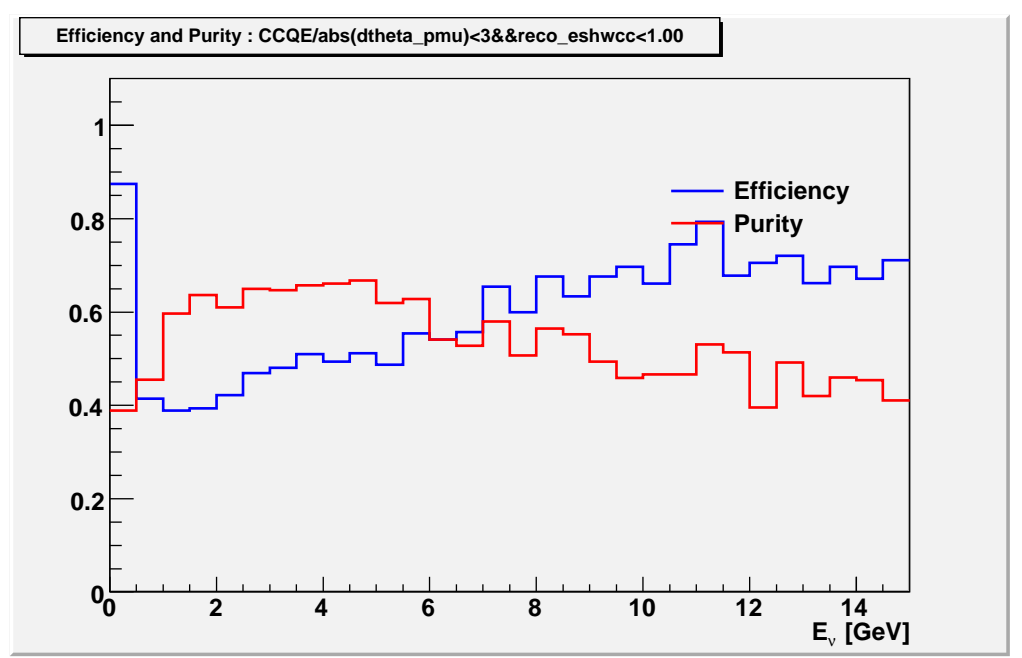

FIG. 47: Far Detector QEL-like event cuts. Purity and efficiency as a function of the energy are shown in red and blue lines respectively. 


\section{Event Distribution/Beam Stability}

Several criteria were applied to check the performance of Far Detector and the quality of CC- and QEL-like event selection procedure. First, track vertex and angular distributions are shown in Figure 48. Track $r^{2}$ - and z-vertex distributions are expected to be flat over the detector. Black and red lines show CC- and QEL-like events. Flat vertex distributions are observed when applying the initial cut and the QEL-like event selection. Track angles must be greater than 0.6 to remove cosmic ray events and distributed pointing to the neutrino beam direction of $3^{\circ}$ upward from the target position. CC- and QEL-like events are clearly peaked at the beam direction.

Second criteria is that the event time distribution relative to the predicted beam spill at the Far Detector must be stable within $50 \mu s$ time window. Figure 49 shows that the selected events are distributed in $50 \mu s$ window. The CC- and QEL-like event numbers with respect to the POT counting are shown as a function of time or run number in Figure 50 . Flat distributions are observed indicates the data are stable over this data taking time period.

\section{Oscillation Fit}

The oscillation fit is performed with Far Detector data sets of $2.50 \times 10^{20}$ POT. The Far Detector expected spectrum is predicted using the best values of the Near Detector fit with the $M_{A}^{R E S}$-scale parameter, which is sensitive to the flux and uncertainties of systematic parameters and not only depends on the $Q^{2}$ distributions, but also $E_{\nu}$ distributions. A mock data oscillation fit result is presented in Appendix E. The details of data fit are as follows : 

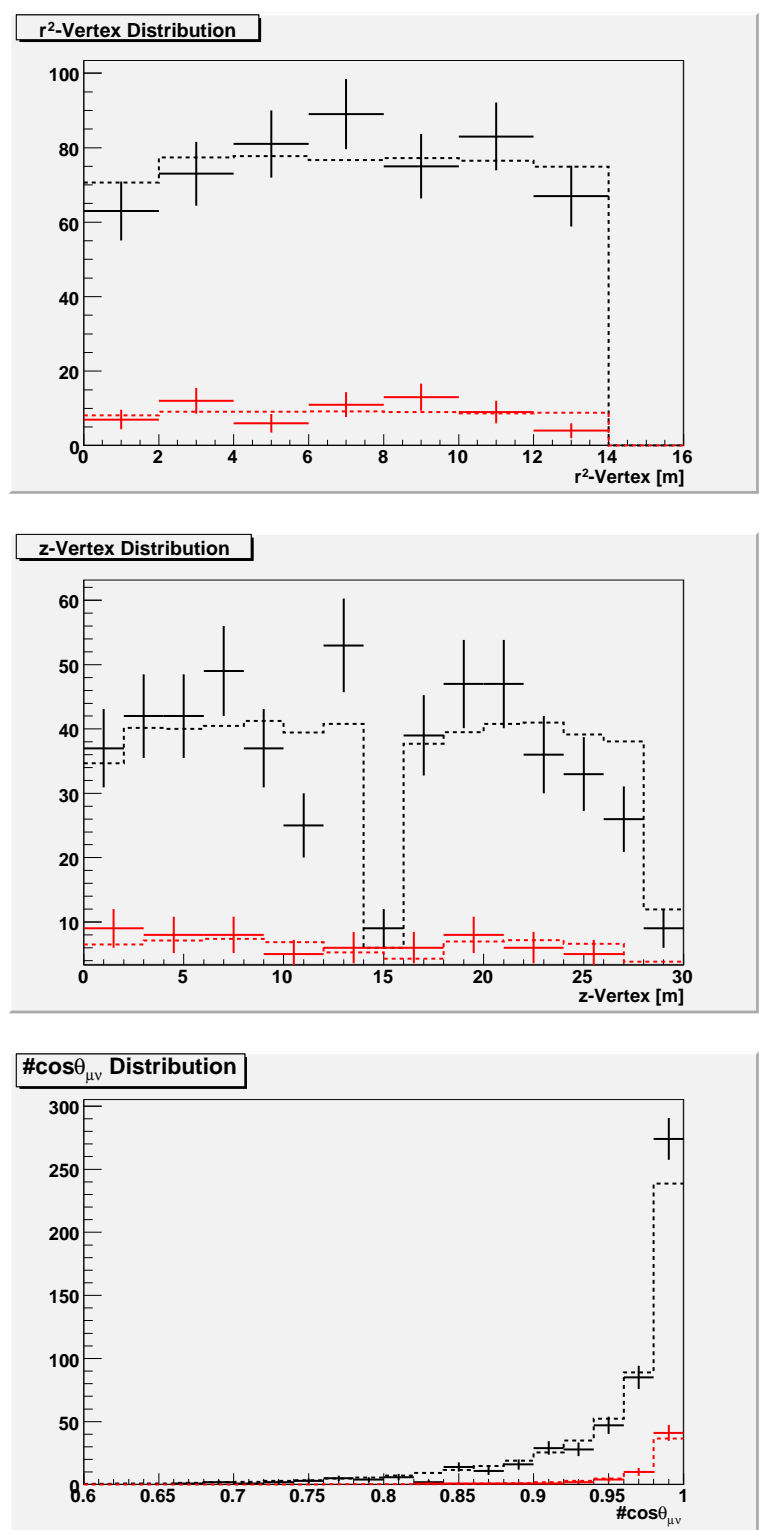

FIG. 48: Far Detector event distributions. $r^{2}$ - and $z$-vertex distributions are shown in top and middle figures. The left bottom figure shows the distributions of the track angles relative to the neutrino beam direction. $\mathrm{MC}$ distributions are normalized to the data event number. 


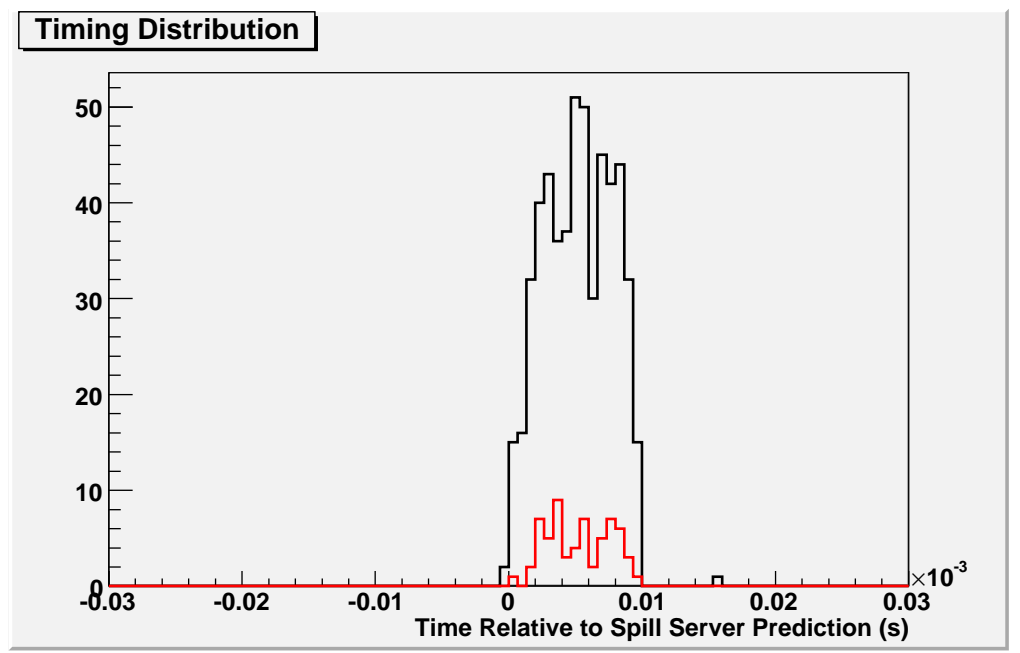

FIG. 49: Event timing distribution. The event timing relative to the predicted beam spill at Far Detector are distributed within $50 \mu \mathrm{s}$ timing window.

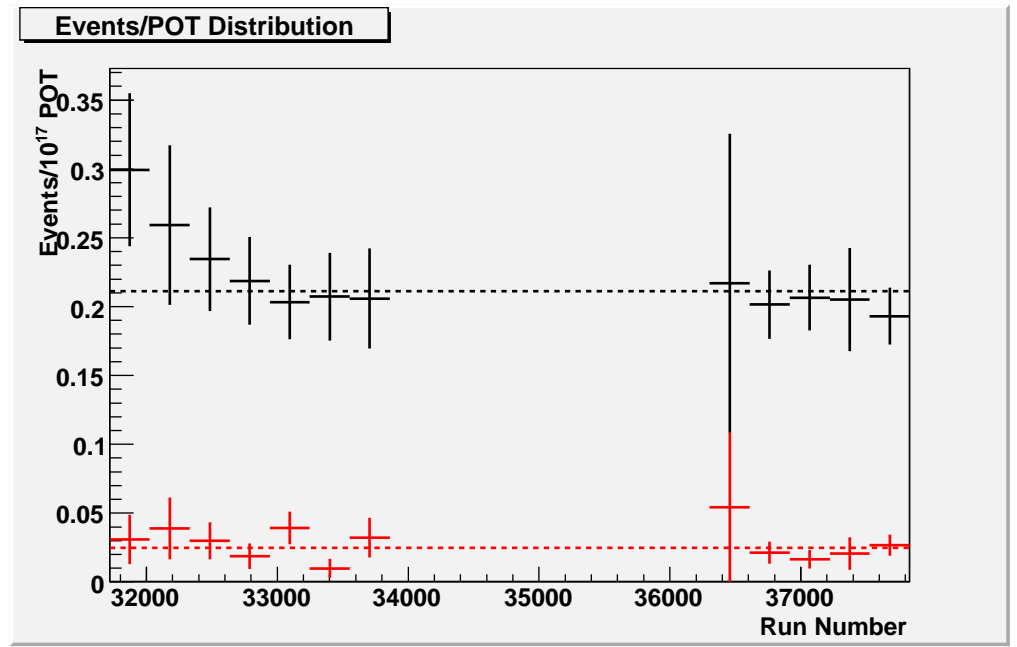

FIG. 50: Beam stability. The CC- and QEL-like event number respect to the POT counting is shown as a function of time or run number. 
$\chi^{2}$ Definition : The statistical minimization function in the fit is defined as follows:

$$
\chi^{2}=\sum_{i=0}^{\text {nbins }} 2\left(N_{i}^{e x p}-N_{i}^{o b s}\right)+2 N_{i}^{o b s} \ln \left(N_{i}^{o b s} / N_{i}^{e x p}\right)+\sum_{j=0}^{\text {nsyst }} \frac{\Delta \alpha_{j}^{2}}{\sigma_{\alpha_{j}}^{2}}
$$

where $N_{i}^{\text {obs }}$ and $N_{i}^{\text {exp }}\left(\Delta m_{32}^{2}, \sin ^{2}\left(2 \theta_{23}\right), \alpha_{0}, \ldots \alpha_{n}\right)$ are the observed and expected number of events at i-th bin. The second term penalizes the value of $\chi^{2}$ as the systematic parameters are varied away from their nominal values. $\alpha_{j}$ are the systematic parameters, with associated errors $\sigma_{\alpha}$ identified in the Near Detector fit. The expected event number is weighted by Equation (2.5) where $L$ and $E$ are the distance between the Near and Far Detectors and neutrino initial energy.

Binning : 10 bins with $1.0 \mathrm{GeV}$ bin width in the range of $E_{\nu}^{Q E} \in[0: 10] \mathrm{GeV}$.

Fit Results : The result of the oscillation parameter fit to data is shown in Figure 51. The fit is constrained to only use values of $\sin ^{2}(2 \theta) \leq 1.0$ in physical region. Equations (2.5) and (2.4) show that the mixing angle is associated to the probability amplitude and sensitive to measure event detection rate and statistics at the maximally oscillated position. The null oscillation hypothesis has a $\chi^{2} / d o f=36.306 / 9$ and is disfavored at $4.93 \sigma$ level. Furthermore, a 1D oscillation fit is performed only with the mass differences while the mixing is constrained to maximal value. The $1 \mathrm{D}$ fit result is shown at Figure 52. The best fit value is $\Delta m^{2}=2.10 \pm 0.37$ (stat.) $\times 10^{-3} \mathrm{eV}^{2}$ with a $\chi^{2} / d o f=13.857 / 10$. The obtained $\Delta m^{2}$ values from those two and one dimensional oscillation fits agree to $\sim 1 \sigma$ level.

Typically the mixing is treated as a free parameter in the oscillation analysis, but the mixing is constrained to the maximal value in the $1 \mathrm{D}$ oscillation fit for the following reason. The $\Delta m^{2}$ measurement is less dependent on statistics and strongly 

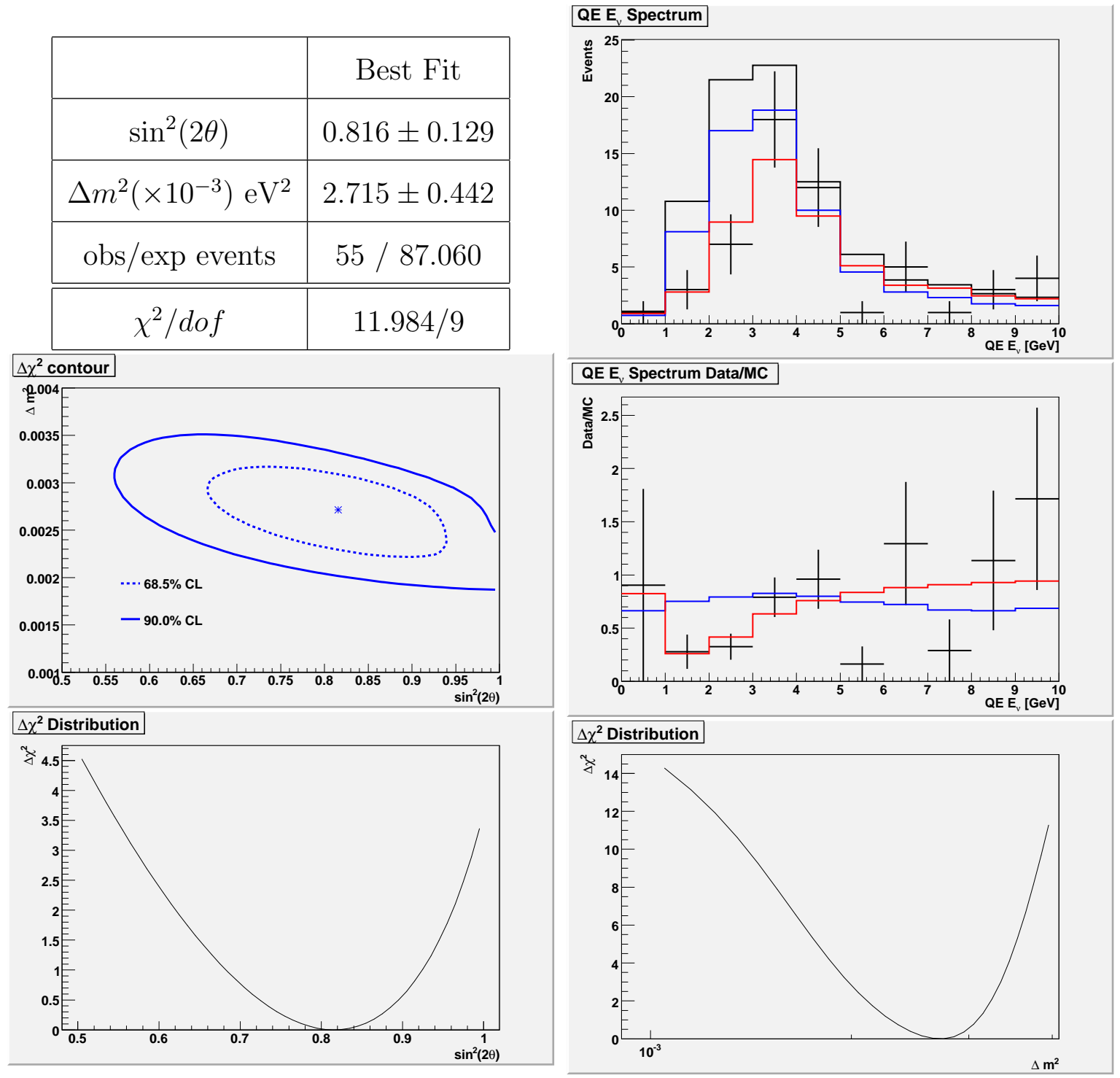

FIG. 51: Far Detector 2D oscillation fit result to the data. Oscillation Analysis result for $2.50 \times 10^{20}$ pot data set, using the Near Detector fit result. Red line is for the best fit oscillated spectrum. Black and blue lines are for the best fit and nominal expected spectra. 

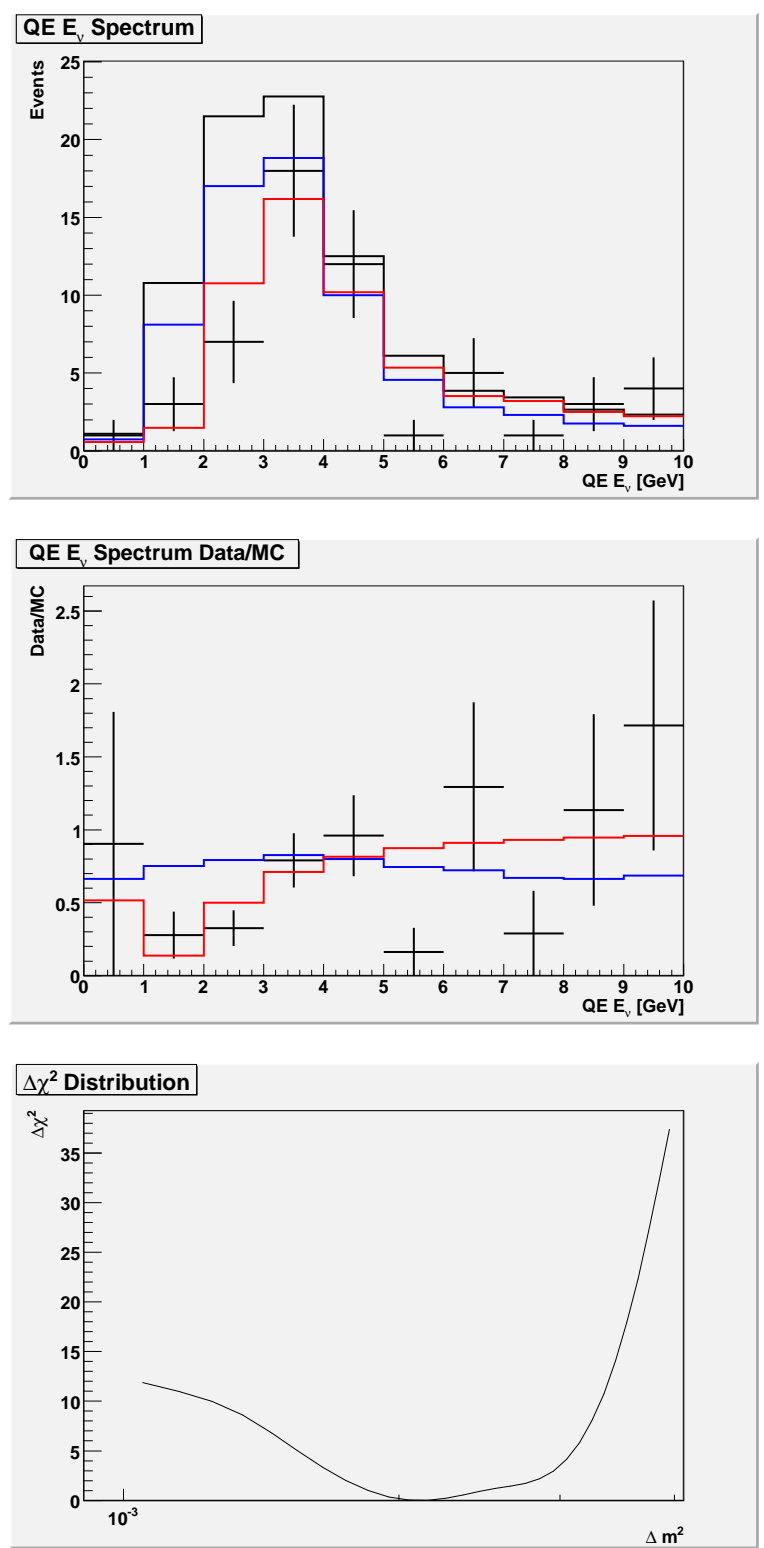

FIG. 52: Far Detector 1D oscillation fit result to the data. Oscillation Analysis result for $2.50 \times 10^{20}$ pot data set, using the Near Detector fit result. Mixing angle is fixed to maximal. Red line is for the best fit oscillated spectrum. Black and blue lines are for the best fit and nominal expected spectra. The $\Delta \chi^{2}$ curve of the $\Delta m^{2}$ parameter respect to the best fit $\Delta m^{2}$ is shown in the bottom figure. 
depends on the beam energy position. As described in the section IV.A, the MINOS high energy data sample is sensitive to constrain the high- $\Delta m^{2}$ region and is expected to improve the upper bound on the value of $\Delta m^{2}$. However, significant improvement of the mixing measurement is not expected performing current and future low statistics QEL analyses. The mixing angle can be more precisely measured with high statistics events. Low statistics data samples from the SK, K2K and MINOS experiments are consistent with the best fit values of the mixing largely fluctuated to the unphysical region and not significantly sensitive to constrain the mixing from the maximal to nonmaximal values [42, 43, 51, 9, 82]. For instance, the MINOS experiment has collected the highest statistics data samples up to $7.5 \times 10^{20} \mathrm{POT}$ and stops taking neutrino data in the fall of 2009. Roughly 1900 CC-like events are estimated to be observed at the Far Detector and are expected to reach the sensitivity of $\sin ^{2}(2 \theta)>0.95$ at $68 \%$ C.L. [46]. Thus these data samples are low statistics and not sensitive to measure the deviation from the maximal mixing. In addition, the upcoming $\mathrm{T} 2 \mathrm{~K}$ and $\mathrm{NO} \nu \mathrm{A}$ experiments $[47,49]$ are planned to operate a 3-5 years neutrino running at the 0.7$0.75 \mathrm{MW}$ beam power and to increase the proton intensity up to $18 \times 10^{20} \mathrm{POT}$. These high statistics data samples will allow for much higher precision measurements of these oscillation parameters. 
Systematic effects : High statistics Far Detector "fake" (or mock) data set is generated applying the best fit values of the oscillation parameters and $\pm 1 \sigma$ systematic errors. The effects of the systematic uncertainties discussed in the section IV.B.5 are evaluated by performing a fit on the modified data set. The difference between the best fit and the values obtained by the modified MC fit is calculated for each systematic parameter and shown in Table III. Those shifts are small and often negligible, comparing to the expected statistical errors for the best fit value of $\Delta m^{2}=2.102 \times 10^{-3} \mathrm{eV}^{2}$ and $\sin ^{2}(2 \theta)=1.00$. The largest systematic effects are found to be the uncertainty of the shower energy offset, and the QEL cross section. The $\Delta m^{2}$ value is sensitive to the uncertainty of the QEL $M_{A}$-scale. The mixing and expected event number are largely influenced by the uncertainty of the shower offset due to the strong shower dependent on the QEL selection procedure. Furthermore, the systematic effect for the $4 \%$ uncertainty of the relative normalization is so small that the data/MC disagreement observed in the Near Detector fit is negligible to the oscillation measurement. The uncertainty is composed from a $2 \%$ uncertainty in the fiducial mass of both detectors, a 3\% uncertainty in the relative Near and Far Detector reconstruction efficiencies, estimated from a visual scan of Near and Far Detector data and Monte Carlo events, and a 1\% uncertainty in the live time. 
Table III: Systematic shift for oscillation parameters. The systematic shifts for the fitted parameters are calculated with MC "fake" (or mock) data samples for $\Delta m^{2}=$ $2.102 \times 10^{-3} \mathrm{eV}^{2}$ and $\sin ^{2}(2 \theta)=1.00$. Systematic shifts for $\Delta m^{2}, \sin ^{2}(2 \theta)$ and the expected event number respect to the $10^{20}$ POT counting are shown in 3rd, 4th and 5 th columns.

\begin{tabular}{|c|c||c|c|c|}
\hline parameters & uncertainty & $\delta\left(\Delta m^{2}\right) \times 10^{-3}$ & $\delta\left(\sin ^{2}(2 \theta)\right)$ & $\delta\left(\right.$ Exp. $\left./ 10^{20} \mathrm{POT}\right)$ \\
\hline Normalization & \pm 0.04 & \pm 0.0458 & \pm 0.0036 & \pm 1.3231 \\
\hline$E_{\mu}$ scale & \pm 0.02 & \pm 0.0318 & \pm 0.0064 & \pm 0.7834 \\
\hline$E_{\text {shw } \text { offset }[\mathrm{GeV}]} \pm 0.050$ & \pm 0.0978 & \pm 0.0368 & \pm 3.5119 \\
\hline$M_{A}^{Q E L}$ scale & \pm 0.15 & \pm 0.1849 & \pm 0.0261 & \pm 2.7326 \\
\hline$M_{A}^{R E S}$ scale & \pm 0.15 & \pm 0.0966 & \pm 0.0046 & \pm 1.6292 \\
\hline$R E S / D I S$-transition scale & \pm 0.0098 & \pm 0.0000 & \pm 0.4000 \\
\hline \multicolumn{2}{|c||}{ Beam } & \pm 0.0079 & \pm 0.0048 & \pm 1.6676 \\
\hline \multicolumn{2}{|c||}{ Sum in quadrature } & \pm 0.2374 & \pm 0.0462 & \pm 5.2694 \\
\hline
\end{tabular}




\section{CHAPTER V}

\section{SUMMARY}

The QEL oscillation analysis is performed using the Far Detector data set of $2.50 \times 10^{20}$ POT for the purpose of improving the measurement of the neutrino oscillations at the low- $\Delta m^{2}$ region. The Far Detector expected spectrum is predicted using the best fit values of the Near Detector analysis. MINOS observes significant suppression in the total number of QEL-like events and a distortion of the Far Detector energy spectrum as expected due to the neutrino oscillations. The 55 QEL-like events are observed at the Far Detector while the $87.06 \pm 13.17$ (syst.) QEL-like events are expected with the null oscillation hypothesis. These data are consistent with $\nu_{\mu}$ disappearance via oscillation with $\Delta m^{2}=2.10 \pm 0.37$ (stat.) \pm 0.24 (syst.) $\mathrm{eV}^{2}$ and the maximal mixing angle. The resulting $68 \%$ and $90 \%$ C.L. intervals for the oscillation parameters $\Delta m^{2}$ and $\sin ^{2}(2 \theta)$ are shown in solid and blue lines in Figure 53 . The allowed $\Delta m^{2}$ region at the maximal mixing is between 1.10 and $3.10 \times 10^{-3} \mathrm{eV}^{2}$ at the $90 \%$ C.L., including the statistical and systematic errors. Figure 53 also shows that the QEL oscillation contour is good agreement with the K2K QEL events allowed $\Delta m^{2}$ region between 1.9 and $3.5 \times 10^{-3} \mathrm{eV}^{2}$ at the $90 \%$ C.L. with a best fit value of $2.8 \times 10^{-3} \mathrm{eV}^{2}$ and $\sin ^{2}(2 \theta)=1.0[71,51]$. Thus an upper bound on the value of $\Delta m^{2}$ at the maximal mixing is improved with MINOS high energy data. 


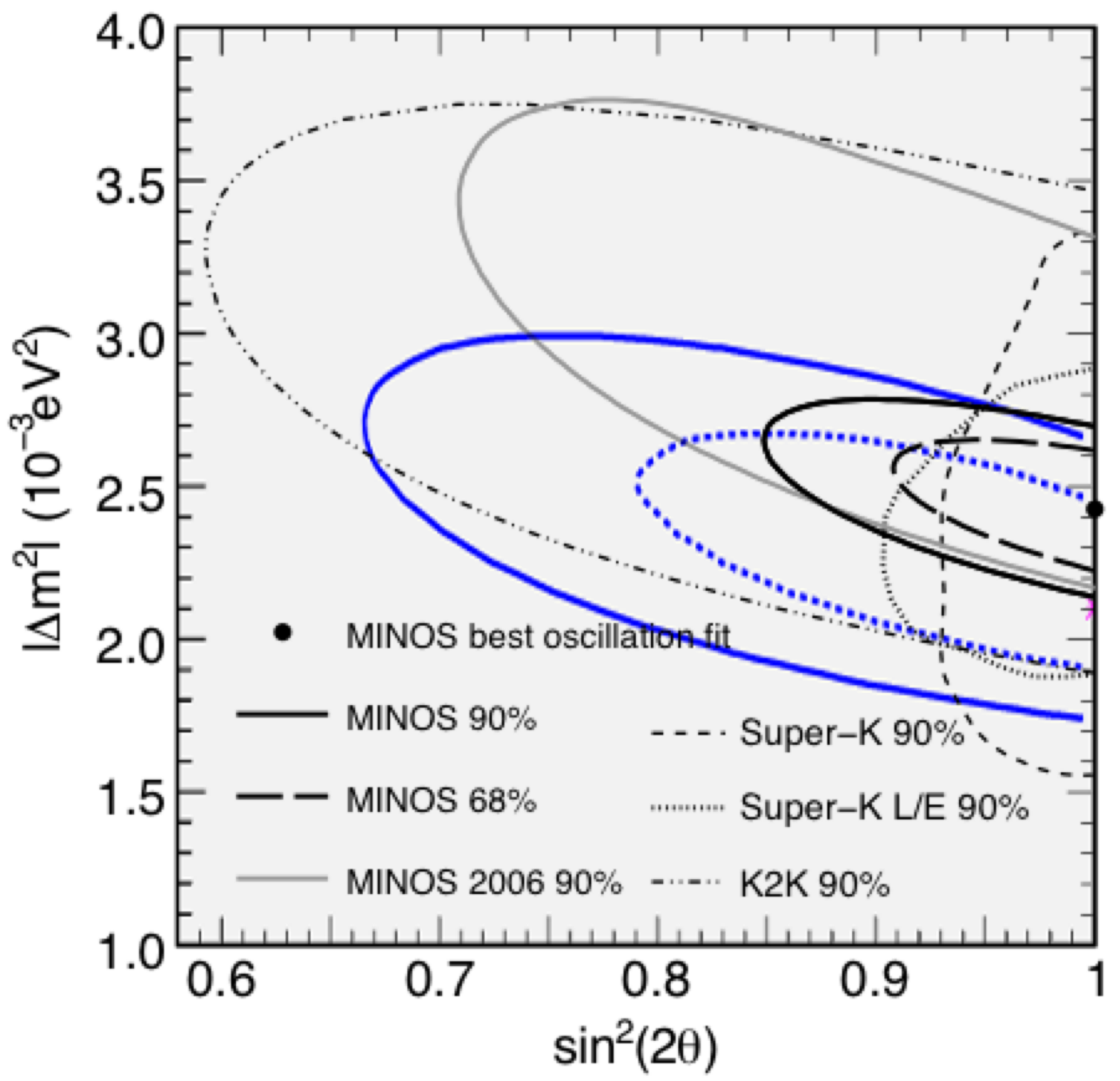

FIG. 53: Comparison of the QEL oscillation contour. The $68 \%$ and 90\% C.L. allowed regions for the oscillation parameters are shown in solid and dashed blue lines. Systematic errors are not included in the contour for the oscillation fit to the QEL data sample. Also shown are contours from previous experiments [42, 43, 71, 51] and MINOS earlier results $[9,45]$. 


\section{REFERENCES}

[1] K. Kobayashi, M. Earl, Y. Ashie, J. Hosaka, K. Ishihara, et al., Phys. Rev. D 72, 052007 (2005).

[2] H. Nishino, S. Clark, K. Abe, Y. Hayato, T. Iida, et al., Phys. Rev. Lett. 102, $141801(2009)$.

[3] S. Mine, J. L. Alcaraz, S. Andringa, S. Aoki, J. Argyriades, et al., Phys. Rev. D 77, 032003 (2008).

[4] N. Cabibbo, Phys. Rev. Lett. 10, 531 (1963).

[5] M. Kobayashi and T. Maskawa, Prog. Theor. Phys. 49, 652 (1973).

[6] C. Amsler, M. Doser, M. Antonelli, D. Asner, K. S. Babu, et al., Phys. Lett. B 667, 1 (2008).

[7] URL http://belle.kek.jp/belle/publications.html.

[8] URL http://www-public.slac.stanford.edu/babar/BaBarPublications.htm.

[9] P. Adamson, C. Andreopoulos, K. E. Arms, R. Armstrong, D. J. Auty, et al., Phys. Rev. Lett. 101, 131802 (2008).

[10] G. Backenstoss, B. D. Hyams, G. Knop, P. C. Marin, and U. Stierlin, Phys. Rev. Lett. 6, 415 (1961).

[11] M. Bardon, P. Franzini, and J. Lee, Phys. Rev. Lett. 7, 23 (1961).

[12] R. N. Mohapatra and P. B. Pal, Massive Neutrinos in Physics and Astrophysics, vol. 72 of World Scientific Lecture Note in Physics (World Scientific, 2004), 3rd ed. 
[13] K. M. Case, Phys. Rev. 107, 307 (1957).

[14] E. Majorana, Nuovo Cimento 14, 171 (1937).

[15] C. Arnaboldi, D. Artusa, F.T.Avignone-III, M. Balata, I. Bandac, et al., Phys. Rev. C 78, 035502 (2008).

[16] H. V. Klapdor-Kleingrothaus, A. Dietz, L. Baudis, G. Heusser, I. V. Krivosheina, et al., Eur. Phys. J. A 12, 147 (2001a).

[17] H. V. Klapdor-Kleingrothaus, A. Dietz, H. L. Harney, and I. V. Krivosheina, Mod. Phys. Lett. A 16, 2409 (2001b).

[18] C. E. Aalseth, F. T. Avignone-III, A. Barabash, F. Boehm, R. L. Brodzinski, et al., Mod. Phys. Lett. A 17, 1475 (2002a).

[19] H. L. Harney, Mod. Phys. Lett. A 16, 2409 (2001).

[20] C. E. Aalseth, F. T. Avignon-III, R. L. Brodzinski, S. Cebrian, E. Garcia, et al., Phys. Rev. D 65, 092007 (2002b).

[21] R. Arnold, C. Augierh, J. Bakerd, A. Barabashf, G. Broudin, et al., Phys. Rev. Lett. 95, 182302 (2005).

[22] Z. Maki, M. Nakagawa, and S. Sakata, Prog. Theor. Phys. 28, 870 (1962).

[23] B. Pontecorvo, J. Exp. Theor. Phys. 28, 984 (1968).

[24] S. R. Elliott, A. A. Hahn, and M. K. Moe, Phys. Rev. Lett. 59, 2020 (1987).

[25] C. Kraus, B. Bornschein, L. Bornschein, J. Bonn, B. Flatt, et al., Eur. Phys. J. 40, 447 (2005). 
[26] A. Osipowicz, H. Blumer, G. Drexlin, K. Eitel, G. Meiselbb, et al., arXiv:hepex/0109033 (2001).

[27] K. Assamagan, C. Brönnimann, M. Daum, H. Forrer, R. Frosch, et al., Phys. Rev. D 53, 6065 (1996).

[28] R. Carey, J. Miller, O. Rind, B. L. Roberts, H. Brown, et al., BNL-E-0952 (2002).

[29] N. Angelov, F. Balestra, Y. Batusov, A. Bianconi, M. Bussa, et al., Nucl. Phys. A $\mathbf{7 8 0}, 78$ (2006).

[30] R. Barate, D. Buskulic, D. Decamp, P. Ghez, C. Goy, et al., Eur. Phys. J. C 2, 395 (1998).

[31] K. Kodama, N. Angelov, N. Ushida, C. Andreopoulos, N. Saoulidou, et al., Phys. Lett. B 504, 218 (2001).

[32] J. Lesgourgues and S. Pastor, Phys. Rep. 429, 307 (2006).

[33] E. Komatsu, J. Dunkley, M. R. Nolta, C. L. Bennett, B. Gold, et al., Astrophys. J. Suppl. Ser. 180, 330 (2009).

[34] B. T. Cleveland, T. Daily, R. Davis, Jr., J. R. Distel, K. Lande, et al., Astrophys. J. 496, 505 (1998).

[35] W. Hampel, J. Handta, G. Heussera, J. Kikoa, T. Kirsten, et al., Phys. Lett. B 447, 127 (1999).

[36] J. N. Abdurashitov, V.N.Gavrin, S.V.Girin, V.V.Gorbachev, P.P.Gurkina, et al., J. Exp. Theor. Phys. 95, 181 (2002).

[37] J. N. Bahcall and M. H. Pinsonneault, Phys. Rev. Lett. 92, 121301 (2004). 
[38] J. N. Bahcall, A. M. Serenelli, and S. Basu, Astrophys. J. 621, L85 (2005).

[39] B. Aharmim, S. Ahmed, A. E. Anthony, E. W. Beier, A. Bellerive, et al., Phys. Rev. C 72, 055502 (2005).

[40] B. Aharmim, S. N. Ahmed, J. F. Amsbaugh, A. E. Anthony, J. Banar, et al., Phys. Rev. Lett. 101, 111301 (2008).

[41] S. Abe, T. Ebihara, S. Enomoto, K. Furuno, Y. Gando, et al., Phys. Rev. Lett. 100, 221803 (2008a).

[42] Y. Ashie, J. Hosaka, K. Ishihara, Y. Itow, J. Kameda, et al., Phys. Rev. Lett. 93, 101801 (2004).

[43] K. Abe, Y. Hayato, T. Iida, M. Ikeda, J. Kameda, et al., Phys. Rev. D 77, 052001 (2008b).

[44] E. Ables, W. W. M. Allison, G. J. Alner, D. S. Ayres, B. Barish, et al., FermilabProposal-0875 (1995).

[45] D. A. Petyt, NuMI-MINOS DocDB/1342-v4,1680-v9,4288-v1,4303-v1,4304-v1 (2006-08).

[46] J. Evans, NuMI-MINOS DocDB/5391-v7 (2008).

[47] Y. Itow, T. Kajita, K. Kaneyuki, M. Shiozawa, Y. Totsuka, et al., KEK report 2001-4, ICRR-report-477-2001-7, TRI-PP-01-05 (2001).

[48] J. A. Thomas and P. L. Vahle, eds., Nuetrino Oscillations Present Status and Future Plan (World Scientific, 2008), 1st ed.

[49] D. S. Ayres, J. W. Dawson, G. Drake, M. C. Goodman, J. J. Grudzinski, et al., Fermilab-Proposal-0929 (2005). 
[50] R. Plunkett, J. Phys.: Conf. Ser. 120, 052044 (2008).

[51] M. H. Ahn, E. Aliu, S. Andringa, S. Aoki, Y. Aoyama, et al., Phys. Rev. D 74, 072003 (2006).

[52] S. Berridge, W. Buggg, J. Busenitza, S. Dazeleye, G. Drake, et al., arXiv:hepex/0410081v1 (2004).

[53] A. Atre, T. Han, S. Pascoli, and B. Zhang, arXiv:hep-ph/0901.3589v1 (2009).

[54] R. N. Mohapatra, S. Antusch, K. Babu, G. Barenboim, M.-C. Chen, et al., Rep. Prog. Phys. 70, 1757 (2005).

[55] C. Athanassopoulos, L. B. Auerbach, R. L. Burman, I. Cohen, D. O. Caldwell, et al., Phys. Rev. Lett. 77, 3082 (1996a).

[56] C. Athanassopoulos, L. B. Auerbach, R. L. Burman, I. Cohen, D. O. Caldwell, et al., Phys. Rev. C 54, 2685 (1996b).

[57] A. Aguilar, L. Auerbach, R. Burman, D. Caldwell, E. Church, et al., Phys. Rev. D 64, 112007 (2001).

[58] A. A. Aguilar-Arevalo, A. O. Bazarko, S. J. Brice, B. C. Brown, L. Bugel, et al., Phys. Rev. Lett. 98, 231801 (2007).

[59] M. Maltoni, J. Phys.: Conf. Ser. 110, 082011 (2008).

[60] M. Maltoni and T. Schwetz, Phys. Rev. D 76, 093005 (2007).

[61] C. Giunti, Mod. Phys. Lett. A 18, 1179 (2003).

[62] A. Pierce and H. Murayama, Phys. Lett. B 581, 218 (2004).

[63] S. Dodelson, A. Melchiorri, and A. Slosar, Phys. Rev. Lett. 97, 04301 (2006). 
[64] S. Goswami and W. Rodejohann, Phys. Rev. D 73, 113003 (2006).

[65] V. Barger, D. Marfatia, and K. Whisnant, Phys. Lett. B 576, 303 (2003).

[66] M. Sorel, J. Conrad, and M. Shaevitz, Phys. Rev. D 70, 073004 (2004).

[67] M. Maltoni, T. Schwetz, M. A. Tortola, and J. W. F. Valle, Nucl. Phys. B 643, 321 (2002).

[68] M. Maltoni, T. Schwetz, M. A. Tortola, and J. W. F. Valle, Nucl. Phys. Proc. Suppl. 114, 203 (2003).

[69] T. Schwetz, arXiv:hep-ph/0805.2234v1 (2008).

[70] A. A. Aguilar-Arevalo, C. E. Anderson, S. J. Brice, B. C. Brown, L. Bugel, et al., arXiv:hep-ex/0904.1958v1 (2008).

[71] E. Aliu, S. Andringa, S. Aoki, J. Argyriades, K. Asakura, et al., Phys. Rev. Lett. 94, $081802(2005)$.

[72] G. P. Zeller, J. Phys.: Conf. Ser. 136, 022028 (2008).

[73] R. Armstrong, M. Ishitsuka, S. Kopp, R. Ospanov, Z. Pavlovic, and P. Vahle, NuMI-MINOS DocDB/4166-v5 (2008).

[74] R. Gran, E. J. Jeon, E. Aliu, S. Andringa, S. Aoki, et al., Phys. Rev. D 74, $052002(2006)$.

[75] A. Blake, NuMI-MINOS DocDB/2953-v1 (2007).

[76] M. Kordosky, AIP Conf. Proc. 896, 185 (2007).

[77] S. Dytman, H. Gallagher, and M. Kordosky, arXiv:hep-ex/0806.2119v1 (2008). 
[78] M. E. Dorman, NuMI-MINOS DocDB/5284-v1 (2008).

[79] C. Andreopoulos, D. Bhattacharya, S. Dytman, H. Gallagher, R. Gran, et al., NuMI-MINOS DocDB/2989-v6 (2007).

[80] H. Gallagher, NuMI-MINOS DocDB/1371-v1 (2006).

[81] S. T. Kumaratunga, NuMI-MINOS DocDB/4610-v1 (2008).

[82] R. Ospanov, NuMI-MINOS DocDB/5544-v1 (2008).

[83] R. N. Bracewell, The Fourier Transformation and Its Applications, Electrical Engineering Series (McGraw-Hill, 2000), 3rd ed. 


\section{APPENDIX A}

\section{APPLICATION OF FOURIER TRANSFORMATION TO THE QEL SELECTION PROCEDURE}

High energy muon can be detected as a track in the MINOS detector, but hadronic particles can be recognized in the various forms of shower energy that spreads around the muon track vertex. The directions that these low energy hadrons travel through space can not be clearly specified with the MINOS detectors. Thus, the MINOS CC-analysis typically treats the shower as a scaler or point-like variable and ignores internal form of shower energy spread. However, the use of the Fourier transformation [83] allows one to replace the point-like energy value with a quantity that includes the spacial distributions of energy in the shower. There are two advantages to apply this transformation. First consider the internal spread of shower energy and the shower energy density as a function of shower strip positions. The function is parameterized in terms of the radial and angular scaling factors of the internal shower spread. The transformation can convert the density function, $\rho(r)$ in position basis

into an associated amplitude, $f(p)$ in momentum basis. Essentially, the $|f(p)|^{2}$ is the probability that the shower spreads around the track vertex under the impact of the internal hadron momentum. The probability function can then be applied to modify the shower energy distribution and to obtain an effect similar to the MC smearing effect [78]. Secondary advantage is through the uncertainty relation. It states that the momentum-position variance product can not be less than a certain minimum value. It is impossible simultaneously to specify arbitrary functions of momentum and position spectra together. The momentum variance and its minimum can be 
uniquely formulated as a function of the internal shower scaling factors. The momentum variance is inversely correlated to the position variance largely dominated with detector's grid size. Thus all physical properties can be converted into better precision in the momentum basis if the uncertainty relation is always minimized under the transformation $\left(\Delta p \approx \frac{1}{4 \pi \Delta x}\right)$. For example, the shower angular variance, $\delta \theta$ is typically calculated in position basis. The shower is most likely to form a bulk of energy around event vertex in the MINOS detector. The shower resolution is fully ambiguous $(\delta \theta \sim \pi)$, but it may be able to reach better precision in momentum space throughout the Fourier transformation. This transformation can be considered in the three stages described below.

1. Point-like shower spread (QEL Kinematic Correction) - Assume that the shower is a point and uniformly distributed at the track vertex. In this stage, the shower is defined in the simplest form. Two QEL kinematic cuts are prepared for selecting the QEL events and depend on proton's momentum. Details are discussed in the section 4.2.2.

2. Global form of the internal shower spread (Explicit Shower Correction) - Consider the global form of the internal shower energy spread and the shower density function that continuously transforms from a point to a track in minimum and

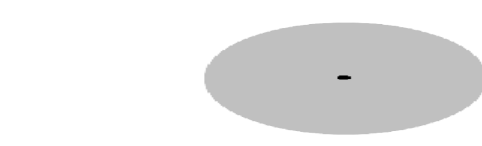

(a) Point

(b) Bulk

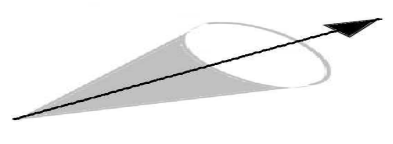

(c) Cone

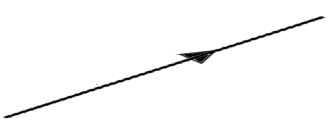

(d) Track

FIG. 54: The transformation of the internal shower spread. Shower is a point and track in minimum and maximum limits. 
maximum limits. The transformation of the shower energy density is shown in Figure 54 .

Apply the Fourier transformation to transform the internal shower distribution in position space to that in momentum space. The transformation can convert measurements in position basis into better precision in momentum basis if the function satisfies minimum relation. The transformation is written in the form of

$$
\text { Point }- \text { like } \Longrightarrow \text { Internal Spread }: f(p)=\int \rho(\vec{r}) e^{i\left(\vec{p}-\vec{p}_{0}\right) \cdot \vec{r}} d \vec{r}
$$

where $\rho(\vec{r})$ is the shower energy density function in position and can be modified from data and MC. The normalization condition is $\int \rho(\vec{r}) d \vec{r}=1 . \vec{p}_{0}$ is at the peak of the density function. The $|f(p)|^{2}$ is the probability that the shower spreads around the track vertex as a function of the internal shower momentum. Finally the $f(p)$ will be applied to modify the kinematic based selection. An example of the shower density function is described in Appendix B.

3. Local shift of the internal shower spread (Nuclear Correction) - The QEL interaction is approximately elastic and energy conserved process. Consider possible nuclear effects to violate the conservation law. Most physics effects will appear as phase shifts in local scale of the density function and not so much effective to change the resolutions, but the intranuclear rescattering effects may be able to largely push up the momentum and angular variances. Possible nuclear effects are listed as follows; (1) the effective binding energy term for the RFG model $\left(m_{n} \Rightarrow m_{n}-\epsilon_{B}\right)$, (2) the Pauli-blocking nuclear effect (free nucleon $\Rightarrow \kappa)$ and $(3)$ the Intranuclear rescattering effect $\left(\rho(\vec{r})_{\text {on }} \Longleftrightarrow \rho(\vec{r})_{\text {off }}\right)$. A detailed exploration of these effects will be the subject of future analyses. 


\section{APPENDIX B}

\section{AN EXAMPLE OF THE SHOWER DENSITY FUNCTION}

Consider symmetric shower spread respect to track vertex. Assume the simple shower density function in position space, $\rho(r)=e^{-M r}$ and do the Fourier transformation to convert it in momentum space. $M$ is the scale factor that represent the size of the shower spread. Detailed calculations are discussed as follows;

1. Do the Fourier transformation for the bulk-like shower energy spread.

$$
\begin{aligned}
f(p) & =\int \rho\left(r^{\prime}\right) e^{i\left(\vec{p}-\overrightarrow{\left.p_{0}\right) \cdot \vec{r}}\right.} d^{3} r=\int \rho(r) e^{i\left(p-p_{0}\right) r \cos \theta} r^{2} d r d \phi^{\prime} \int_{-1}^{1} d \cos \theta \\
& =2 \pi \int \rho\left(r^{\prime}\right) e^{i\left(p-p_{0}\right) r \cos \theta} r^{2} d r \int_{-1}^{1} d \cos \theta \\
& =2 \pi \int \rho\left(r^{\prime}\right) \frac{e^{i\left(p-p_{0}\right) r}-e^{-i\left(p-p_{0}\right) r}}{2 i\left(p-p_{0}\right) r} 2 r^{2} d r \\
& =\left(\frac{4 \pi}{p-p_{0}}\right) \int \rho(r) \sin \left(\left(p-p_{0}\right) r\right) r d r \\
& =\left(\frac{4 \pi}{p-p_{0}}\right) \int_{0}^{\infty} e^{-M r} \sin \left(\left(p-p_{0}\right) r\right) r d r=\frac{\left(\frac{4 \pi}{M^{3}}\right)}{\left(1+\left(\frac{p-p_{0}}{M}\right)^{2}\right)^{2}}
\end{aligned}
$$

where the constant term in numerator will be vanished due to normalization condition $\int \rho\left(r^{\prime}\right) d^{3} r^{\prime}=1$. Finally the density function in momentum and position spaces can be written in the form of

$$
\begin{aligned}
& f(p)=\frac{f\left(p_{0}\right)}{\left(1+\left(\frac{p-p_{0}}{M}\right)^{2}\right)^{2}} \\
& \rho(r)=\rho_{0} e^{-M r}
\end{aligned}
$$

where $f\left(p_{0}\right)$ and $\rho_{0}$ are normalization factors. Note that the shower is symmetrically distributed around the event vertex. Figure 55 shows the $\rho(r)$ and $f(p)$ distribution when varying the $M$-scale. 
2. The uncertainty relation can be converted as follows;

$$
(\Delta r)^{2}=<r^{2}>-<r>^{2}=\frac{f^{\prime \prime}\left(p_{0}\right)}{-4 \pi^{2} f\left(p_{0}\right)}+\frac{1}{4 \pi^{2}}\left[\frac{f^{\prime}\left(p_{0}\right)}{f\left(p_{0}\right)}\right]
$$

where $f^{\prime}\left(p_{0}\right)$ is canceled out and $f^{\prime \prime}\left(p_{0}\right)$ is calculated as follows;

$$
f^{\prime \prime}\left(p_{0}\right)=\left.\frac{\partial^{2}}{\partial p^{2}}\left(\frac{f\left(p_{0}\right)}{\left(1+\left(\frac{p-p_{0}}{M}\right)^{2}\right)^{2}}\right)\right|_{p=p_{0}}=f\left(p_{0}\right)(-4)\left(\frac{2}{M}\right)^{2}
$$

If inserting it to the position variance, then

$$
(\Delta r)^{2}=\frac{f\left(p_{0}\right)(-4)\left(\frac{1}{M}\right)^{2}}{-4 \pi^{2} f\left(p_{0}\right)}=\left(\frac{1}{\pi M}\right)^{2}
$$

Thus, the position variance is written in the form of

$$
\Delta r=\frac{1}{\pi M}
$$

If inserting the position variance to the uncertainty relation, then the momentum variance can be converted to

$$
\Delta p \geq \frac{1}{4 \pi \Delta r}=\frac{1}{4 \pi \frac{1}{\pi M}}=\frac{1}{4} M
$$

Thus, the momentum variance is parameterized in terms of the M-scale and angular factors. It is minimized if the relation takes equivalent. Minimum value of the M-scale factor is approximately $M \sim \frac{1}{r_{0}}$. The momentum uncertainty is estimated as follows;

$$
\Delta p \gtrsim \frac{1}{4 r_{0}} \approx \frac{1}{4 \sqrt{\left(2.54+2.40+\frac{1.0}{2}\right)^{2}+\left(\frac{4.10 \sqrt{2}}{2}\right)^{2}}} \approx 0.043
$$


where $r_{0}$ is the detector grid size in an unit of $\mathrm{cm}$, that is converted by $2.54 \mathrm{~cm}$ steel and $4.10 \mathrm{~cm} \times 1.00 \mathrm{~cm}$ scintillation plates, and $2.40 \mathrm{~cm}$ air gap. First estimated minimum value is approximately consistent to the minimum value in Equation (4.4). Most physics effects appear as phase shift in local scale of the density function and not so much effective to change the M-scale factor. But, the intranuclear rescattering effects may be able to largely push up the M-scale factor. More physics effects will be studied in detail at the 3rd stage.
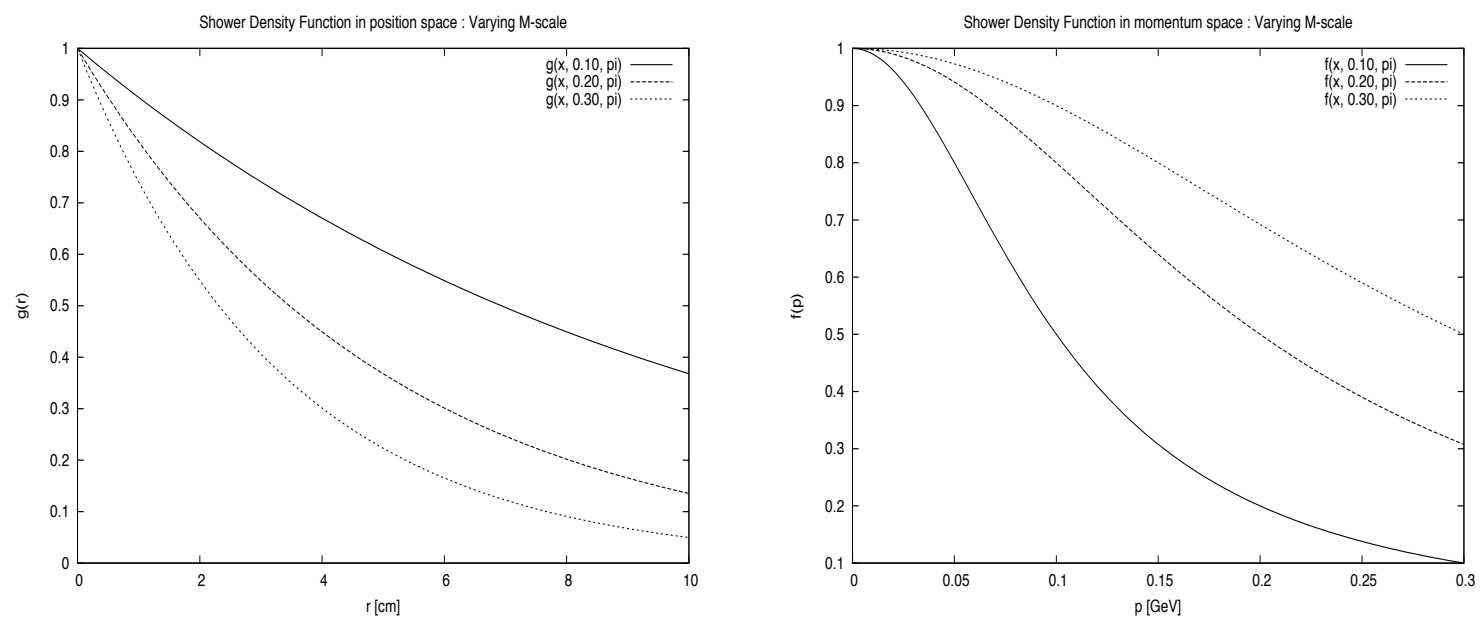

FIG. 55: Example for shower density function. Set $f\left(p_{0}\right)=\rho_{0}=1.0$ for fixed shower energy. Left and right figures show the $\rho(r)$ and $f(p)$ when varying the M-scale. Changing the $M$-scale is sensitive to change the function width respect to position and momentum. Applying the $|f(p)|^{2}$ is expected to change the shower energy and $\delta \theta$-distribution. 


\section{APPENDIX C}

\section{DERIVATION OF FORMULA}

Derivation of the QEL kinematic formula is shown in this Appendix. All formula calculations start from

$$
p_{\nu}+p_{n} \approx p_{p}+p_{\mu}
$$

where $p_{\nu}=\left(E_{\nu}, \vec{p}_{\nu}\right), p_{n}=\left(m_{n}, 0\right), p_{p}=\left(E_{p}, \vec{p}_{p}\right)$ and $p_{\mu}=\left(E_{\mu}, \vec{p}_{\mu}\right)$ are neutrino, neutron, proton and muon four-momentum in the lab frame.

\section{Neutrino Energy}

$$
\begin{aligned}
& p_{\nu}+p_{n}-p_{\mu}=p_{p} \\
& \left(p_{\nu}+p_{n}-p_{\mu}\right)^{2}=p_{p}^{2} \\
& m_{\nu}^{2}+2 p_{\nu} p_{n}+m_{n}^{2}-2 p_{\nu} p_{\mu}-2 p_{n} p_{\mu}+m_{\mu}^{2}=m_{p}^{2} \\
& 2 E_{\nu} m_{n}+m_{n}^{2}-2 E_{\nu}\left(E_{\mu}-\left|\vec{p}_{\mu}\right| \cos \theta_{\nu \mu}\right)-2 m_{n} E_{\mu}+m_{\mu}^{2}=m_{p}^{2} \\
& 2 E_{\nu}\left(m_{n}-E_{\mu}+\left|\vec{p}_{\mu}\right| \cos \theta_{\nu \mu}\right)=2 m_{n} E_{\mu}-\left(m_{\mu}^{2}+m_{n}^{2}-m_{p}^{2}\right)
\end{aligned}
$$

$$
E_{\nu}=\frac{m_{n} E_{\mu}-\left(m_{\mu}^{2}+m_{n}^{2}-m_{p}^{2}\right) / 2}{m_{n}-E_{\mu}+\left|\vec{p}_{\mu}\right| \cos \theta_{\nu \mu}}
$$




\section{Proton-Muon Opening Angle}

$$
\begin{aligned}
& \left(p_{\nu}+p_{n}\right)^{2}=\left(p_{p}+p_{\mu}\right)^{2} \\
& m_{\nu}^{2}+2 p_{\nu} p_{n}+m_{n}^{2}=m_{\mu}^{2}+2 p_{\mu} p_{p}+m_{p}^{2} \\
& 2 E_{\nu} m_{n}+m_{n}^{2}=m_{\mu}^{2}+2\left(E_{\mu} E_{p}-\left|\vec{p}_{\mu}\right|\left|\vec{p}_{p}\right| \cos \theta_{p \mu}\right)+m_{p}^{2} \\
& E_{\mu} E_{p}-\left|\vec{p}_{\mu}\right|\left|\vec{p}_{p}\right| \cos \theta_{p \mu}=E_{\nu} m_{n}-\left(m_{\mu}^{2}+m_{p}^{2}-m_{n}^{2}\right) / 2
\end{aligned}
$$

Set $m_{\mu}^{\prime 2}=m_{\mu}^{2}+m_{p}^{2}-m_{n}^{2}$

$\left|\vec{p}_{\mu}\right|\left|\vec{p}_{p}\right| \cos \theta_{p \mu}=E_{\mu} E_{p}-E_{\nu} m_{n}+m_{\mu}^{\prime 2} / 2$

$$
\cos \theta_{p \mu}=\frac{E_{\mu} E_{p}-E_{\nu} m_{n}+m_{\mu}^{\prime 2} / 2}{\left|\vec{p}_{\mu}\right|\left|\vec{p}_{p}\right|}
$$

\section{Invariant Mass}

$$
\begin{aligned}
& W^{2}=\left(p_{n}+q\right)^{2} \\
& =m_{n}^{2}+2 p_{n} q+q^{2}=m_{n}^{2}+2 p_{n} q-Q^{2} \\
& =m_{n}^{2}+2 p_{n}\left(p_{\nu}-p_{\mu}\right)-Q^{2}=m_{n}^{2}+2 m_{n}\left(E_{\nu}-E_{\mu}\right)-Q^{2} \\
& =m_{n}^{2}+2 m_{n} E_{s h w}-Q^{2}
\end{aligned}
$$

$$
W^{2}=m_{n}^{2}+2 m_{n} E_{s h w}-Q^{2}
$$

Convert proton's energy. The $W^{2}$ can be written in the other form. 


$$
\begin{aligned}
& W^{2}=m_{n}^{2}+2 p_{n} q-Q^{2} \\
& =m_{n}^{2}+2 p_{n}\left(p_{p}-p_{n}\right)-Q^{2} \\
& =m_{n}^{2}+2 m_{n} E_{p}-2 m_{n}^{2}-Q^{2}
\end{aligned}
$$

$$
E_{p}=\frac{m_{n}^{2}+W^{2}+Q^{2}}{2 m_{n}}
$$

If inserting the $W^{2}$, then

$$
\begin{aligned}
& E_{p}=\frac{m_{n}^{2}+W^{2}+Q^{2}}{2 m_{n}} \\
& =\frac{1}{2 m_{n}}\left(m_{n}^{2}+m_{n}^{2}+2 m_{n} E_{\text {shw }}\right)
\end{aligned}
$$

$$
E_{p}=m_{n}+E_{s h w} \text { (shower dependent form) }
$$

If $W^{2} \rightarrow m_{p}^{2}$, then

$$
E_{p}=\frac{m_{n}^{2}+m_{p}^{2}+Q^{2}}{2 m_{n}} \text { (muon dependent form) }
$$

\section{Momentum Transfer}

$$
\begin{aligned}
& Q^{2}=-q^{2}= \\
& =-\left(p_{\nu}-p_{\mu}\right)^{2}=-m_{\nu}^{2}+2 p_{\nu} p_{\mu}-m_{\mu}^{2}
\end{aligned}
$$




$$
Q^{2} \approx 2 E_{\nu}\left(E_{\mu}-\left|\vec{p}_{\mu}\right| \cos \theta_{\mu \nu}\right)-m_{\mu}^{2} \text { (muon dependent form) }
$$

In the other form

$$
\begin{aligned}
& =-\left(p_{p}-p_{n}\right)^{2}=-m_{p}^{2}+2 p_{p} p_{n}-m_{n}^{2} \\
& =-m_{p}^{2}+2 m_{n} E_{p}-m_{n}^{2}
\end{aligned}
$$

Insert $E_{p}=m_{n}+E_{s h w}$

$=-m_{p}^{2}+2 m_{n}\left(m_{n}+E_{s h w}\right)-m_{n}^{2}=2 m_{n}^{2}-m_{p}^{2}-m_{n}^{2}+2 m_{n} E_{s h w}$

$$
Q^{2} \approx 2 m_{n} E_{s h w} \text { (shower dependent form) }
$$




\section{APPENDIX D}

\section{NEAR DETECTOR FIT}

Four parameters are included in the Near Detector fit. The $M_{A}^{Q E L}$ parameter is treated as a free parameter. Systematic parameters are the $E_{\mu}$-scale, $E_{s h w}$-offset, NonQE background scale and $M_{A}^{Q E L}$-scale. The NonQE background spectrum is normalized by the NonQE background event scale. Two fit configurations are prepared for the Near Detector fit. (1) The shape-only $Q^{2}$ fit is configured to use the $Q^{2}$ distribution normalized by the area equally integrated by the histogram bin contents. It takes the advantages that the flux and systematic uncertainties are less sensitive to the shape of the $Q^{2}$ distributions. (2) The shape+rate $Q^{2} / E_{\nu}^{Q E}$ simultaneous fit is configured to use both of the $Q^{2}$ and $E_{\nu}$ distributions that are absolutely normalized by the POT counting. Note that the MC smearing effects to smooth out the discontinuous $\chi^{2}$ surface is not included in those fits [78]. Mock and read data fit results are shown as follows :

Mock data fit results : Mock data analysis is performed for the purpose of testing the ability of the QEL cross section and oscillation parameter fitting procedures and the extrapolation method to predict correct Far Detector expected spectrum from the Near Detector spectrum. The Near Detector mock data is generated with the true values of the systematic parameters; 0.980 in the $E_{\mu}$-scale, $-0.035 \mathrm{GeV}$ in the

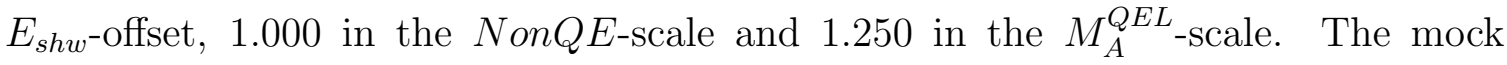
data fit result is shown in Table IV. The shape-only fit result is shown in second column of the table. The best fit value is $M_{A}^{Q E L}=1.208 \mathrm{GeV}$ and well agrees to $\chi^{2}=14.354 / 17$. The $E_{\nu}$ and $Q^{2}$ distributions, and the parameter $\Delta \chi^{2}$ curves respect 
to the shape-only best fit value are shown in Figures 56 and 57. Figure 58 shows the parameter correlations. The $M_{A}^{Q E L}$-scale is not correlated to the $E_{\mu}$-scale, $E_{\text {shw }}$-offset and NonQE background scale while the $M_{A}^{Q E L}$-scale is largely anti-correlated to the background scale. The $E_{\mu}$-scale is largely anti-correlated to the $E_{\text {shw-offset, due to }}$ the strong shower and muon energy dependency in the QEL kinematic selection. The background scale are more likely correlated to the cross section parameters, not to the muon and shower energy scales. Furthermore, the shape+rate fit result is shown in third column of the table. The best fit value is $M_{A}^{Q E L}=1.209 \mathrm{GeV}$ and well agrees to $\chi^{2}=34.667 / 38$. The $E_{\nu}$ and $Q^{2}$ distributions, and the parameter $\Delta \chi^{2}$ curves respect to the shape-only best fit value are shown in Figures 59 and 60. Figure 61 shows the parameter correlations and that the $M_{A}^{Q E L}$-scale is largely correlated to the other parameters. 
Table IV: Near Detector mock data fit results. Four parameters are included in the Near Detector shape-only and shape+rate fits. The Near Detector mock data set is generated with the detector and cross section parameters and SKZP reweighted beam spectrum. The minimum values of the $\chi^{2}$ are less than degree of freedom.

\begin{tabular}{|c|c|c|c|}
\hline & True & shape-only fit & rate+shape fit \\
\hline \multicolumn{2}{|l|}{$Q^{2}$ cut } & \multicolumn{2}{|c|}{$Q^{2}>0.2 \mathrm{GeV}^{2}$} \\
\hline \multicolumn{2}{|l|}{$\mathrm{MC}$} & \multicolumn{2}{|c|}{$0.20057 \times 10^{20} \mathrm{pot}$} \\
\hline \multicolumn{2}{|l|}{ Mock Data } & \multicolumn{2}{|c|}{$0.19418 \times 10^{20}$ pot } \\
\hline \multicolumn{4}{|c|}{ Fit Result } \\
\hline \multicolumn{2}{|l|}{ Obs. Events } & \multicolumn{2}{|c|}{19690.3} \\
\hline \multicolumn{2}{|l|}{ Exp. Events } & 19311.5 & 19577.1 \\
\hline \multicolumn{2}{|c|}{ Obs/Exp Events } & 1.01962 & 1.00578 \\
\hline$E_{\mu}$ scale & 0.980 & 0.988 & 0.979 \\
\hline$E_{\text {shw }}$ offset $[\mathrm{GeV}]$ & -0.035 & -0.0358 & -0.0332 \\
\hline NonQE Background & 1.000 & 1.000 & 1.013 \\
\hline$M_{A}^{Q E L}$ scale & 1.250 & 1.196 & 1.197 \\
\hline \multicolumn{2}{|l|}{$\chi^{2}\left(E_{\nu}\right)$} & $N / A$ & 18.351 \\
\hline \multicolumn{2}{|l|}{$\chi^{2}\left(Q^{2}\right)$} & 13.469 & 14.654 \\
\hline \multicolumn{2}{|l|}{$\chi^{2}$ (syst.) } & 0.885 & 1.662 \\
\hline \multicolumn{2}{|l|}{$\chi^{2}($ total $) / \operatorname{dof}$} & $14.354 / 17$ & $34.667 / 38$ \\
\hline
\end{tabular}




\section{- Shape-only Fit Result -}
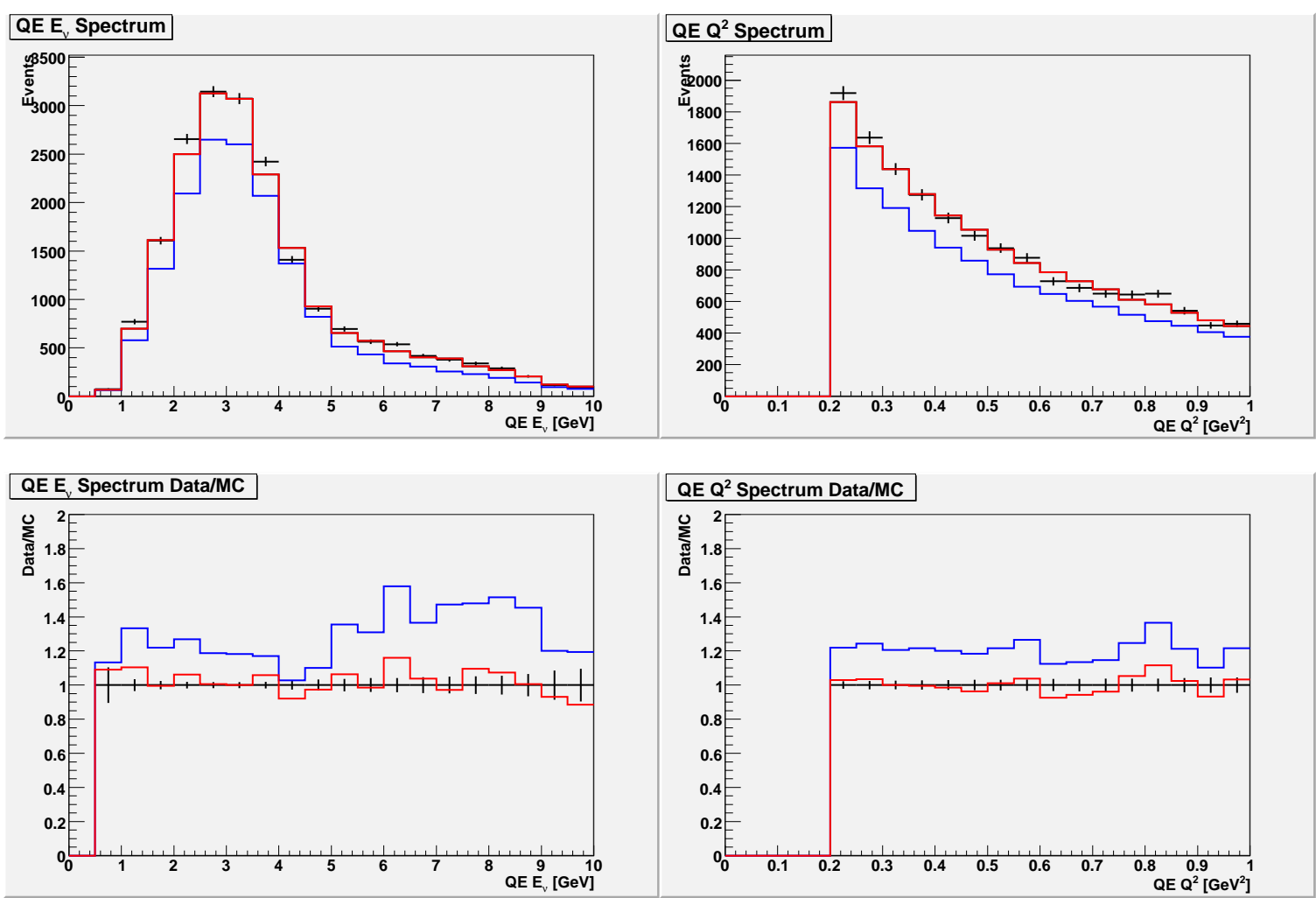

FIG. 56: The $E_{\nu}^{Q E L}$ and $Q^{2}$ distributions of the shape-only fit to the mock data. Blue and red lines show nominal and best fit spectra. 


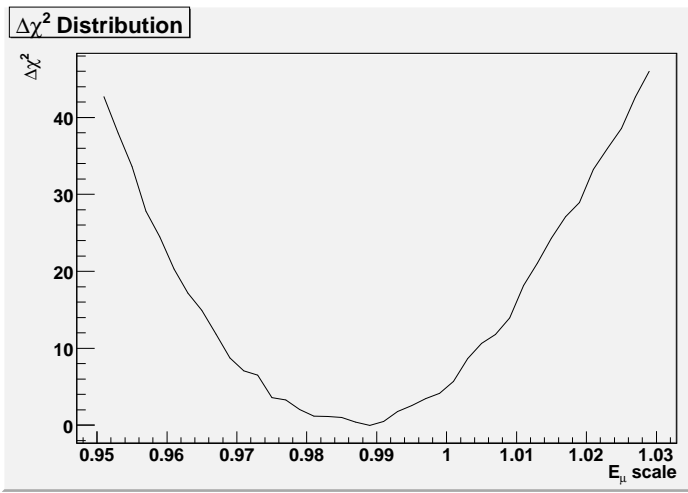

(a) $E_{\mu}$-scale

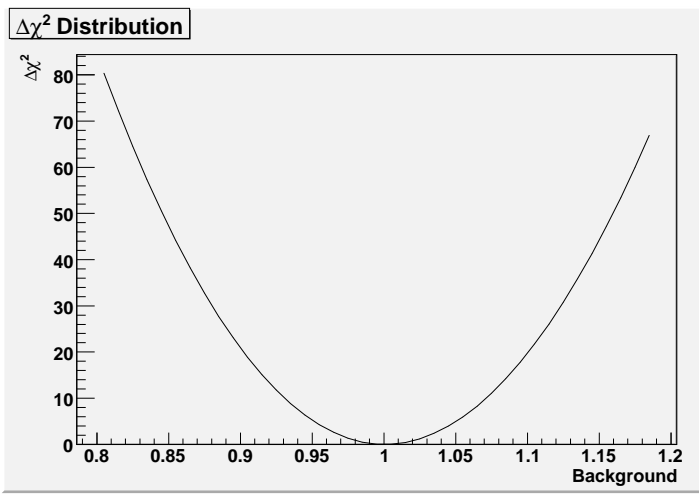

(c) NonQE-scale

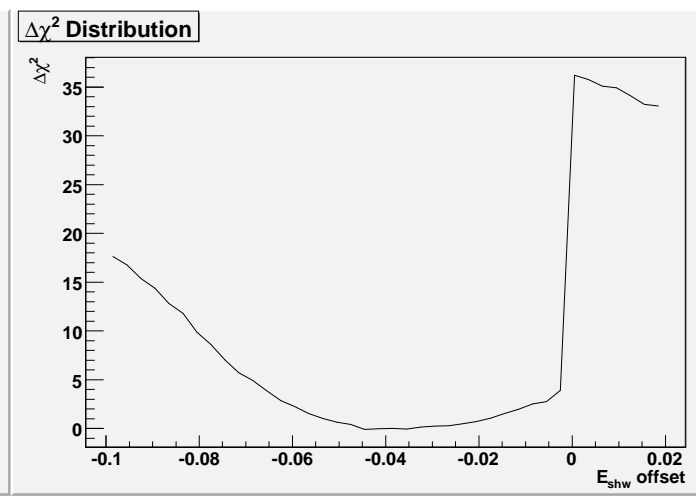

(b) $E_{s h w}$-offset

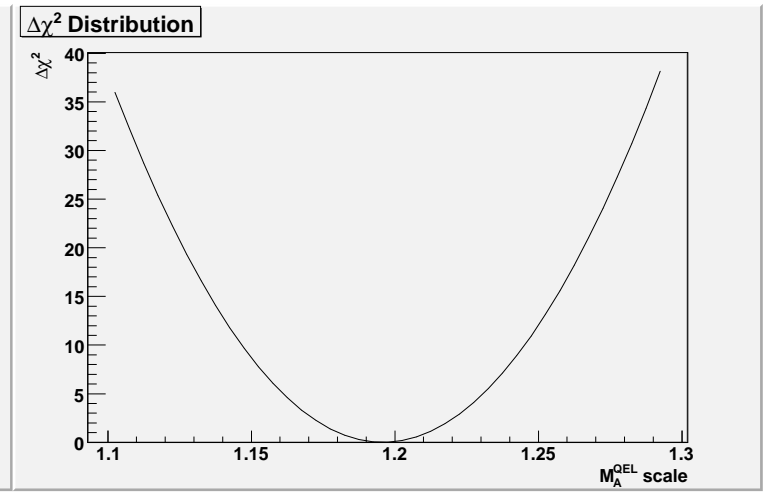

(d) $M_{A}^{Q E L}$-scale

FIG. 57: The parameter $\Delta \chi^{2}$ curves of the shape-only fit to the mock data. 


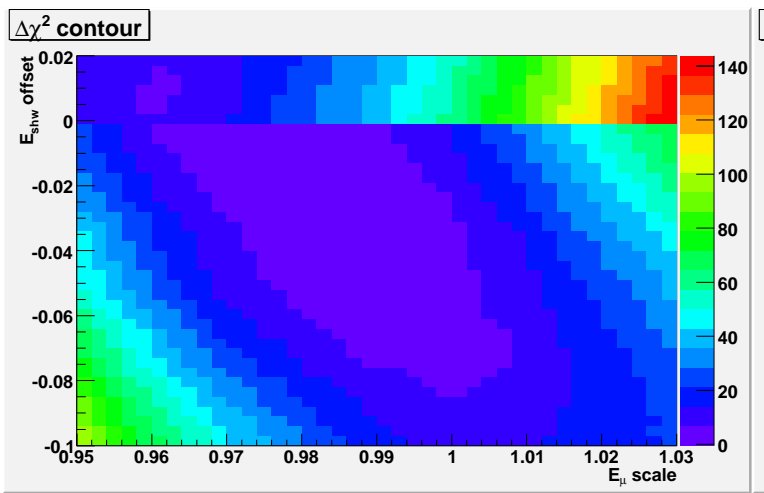

(a) $E_{\mu}$-scale vs $E_{s h \text {-offset }}$

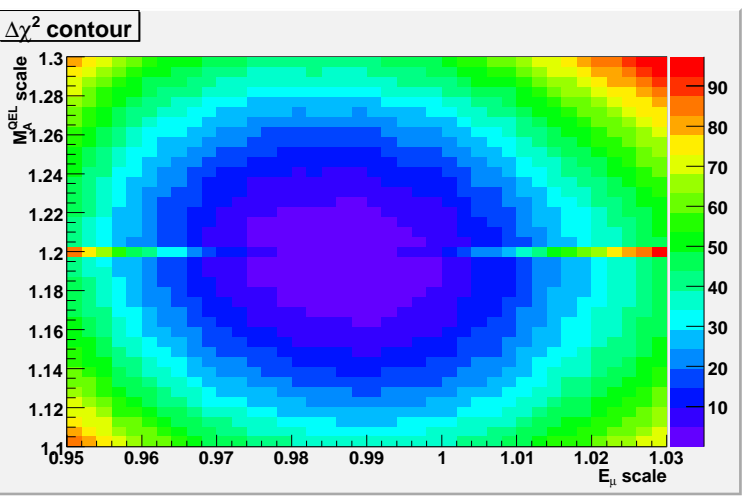

(b) $E_{\mu}$-scale vs $M_{A}^{Q E L}$-scale

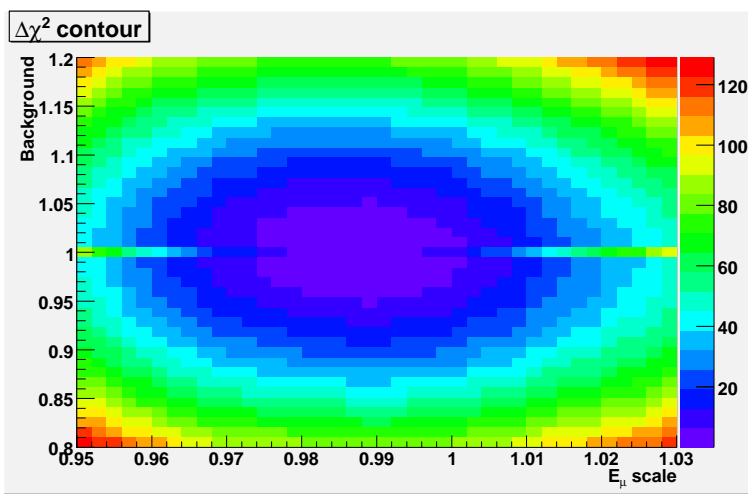

(c) $E_{\mu}$-scale vs NonQE-scale

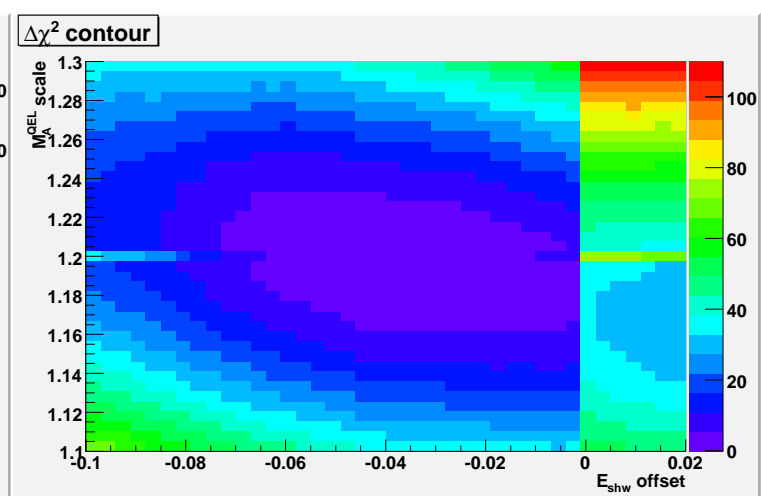

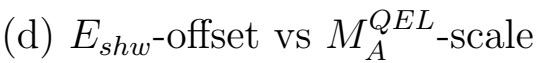

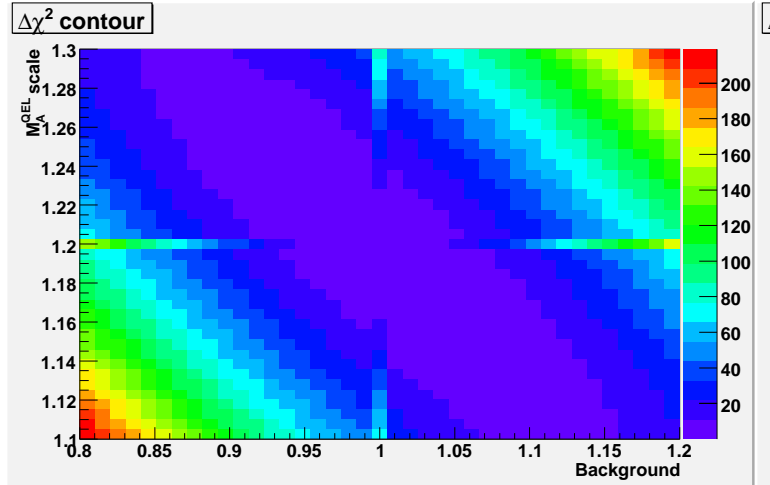

(e) NonQE-scale vs $M_{A}^{Q E L}$-scale

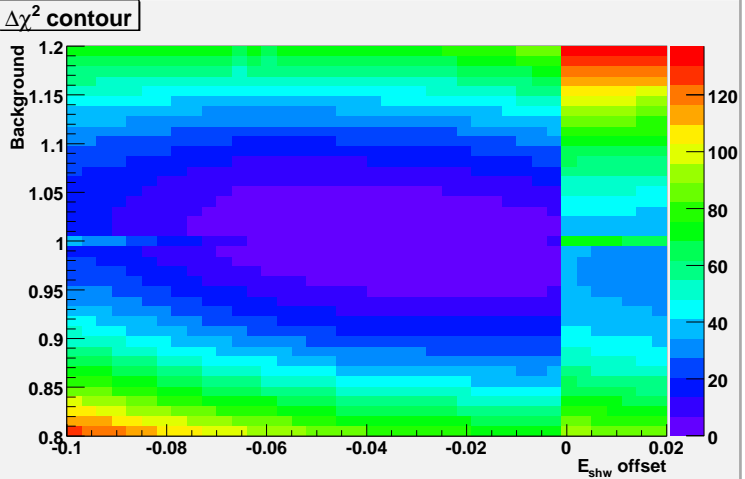

(f) $E_{s h w}$-offset vs $N o n Q E$-scale

FIG. 58: The parameter correlations of the shape-only fit to the mock data. 


\section{— Shape+Rate Fit Result —}
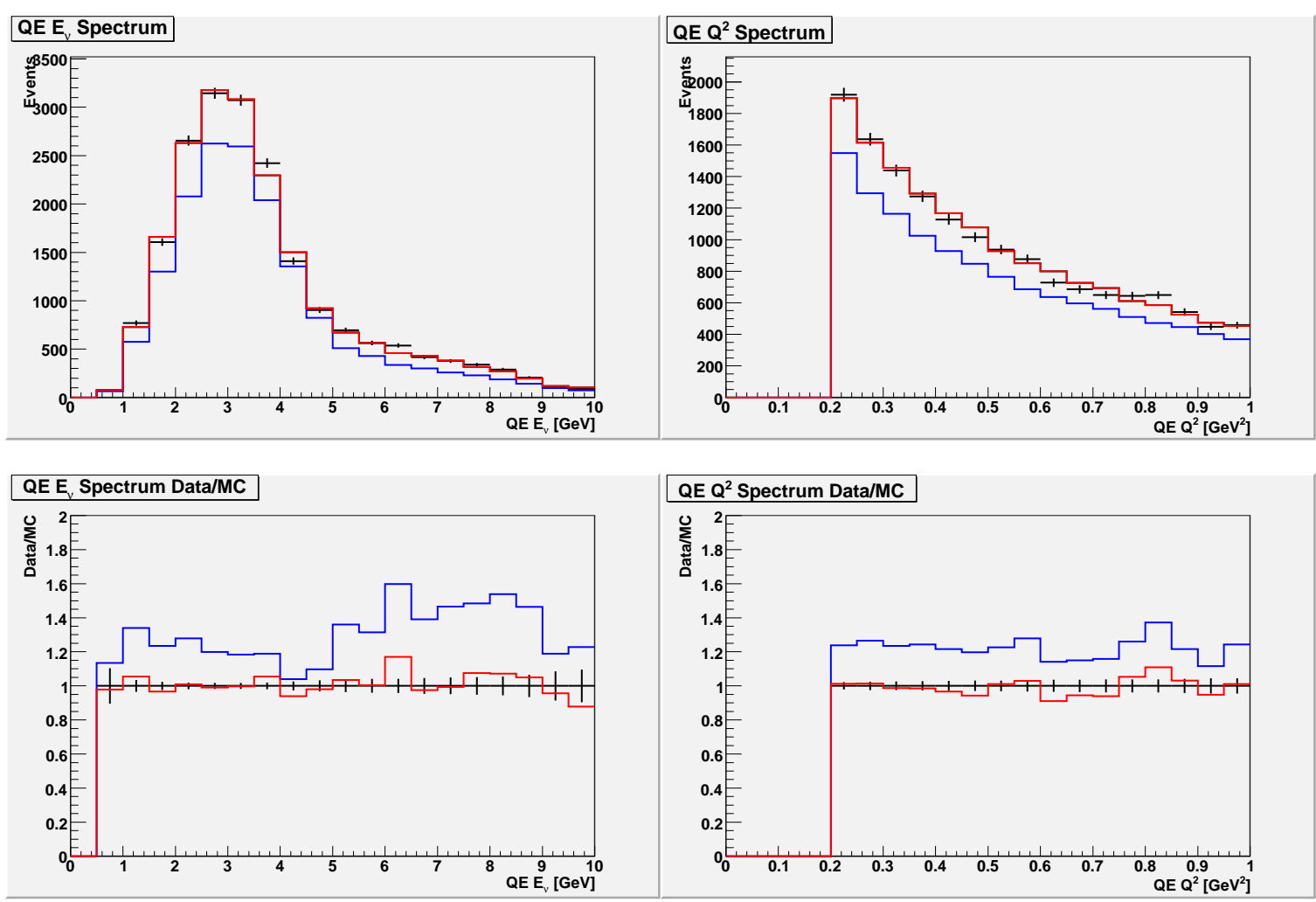

FIG. 59: The $E_{\nu}^{Q E L}$ and $Q^{2}$ distributions of the shape+rate fit to the mock data. Blue and red lines show nominal and best fit spectra. 


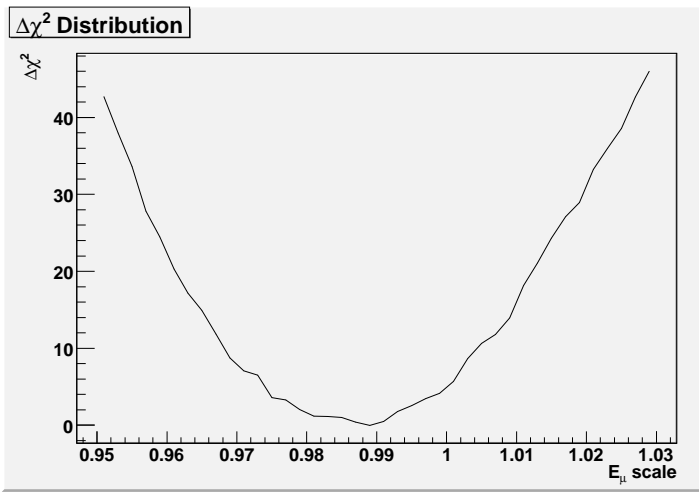

(a) $E_{\mu}$-scale

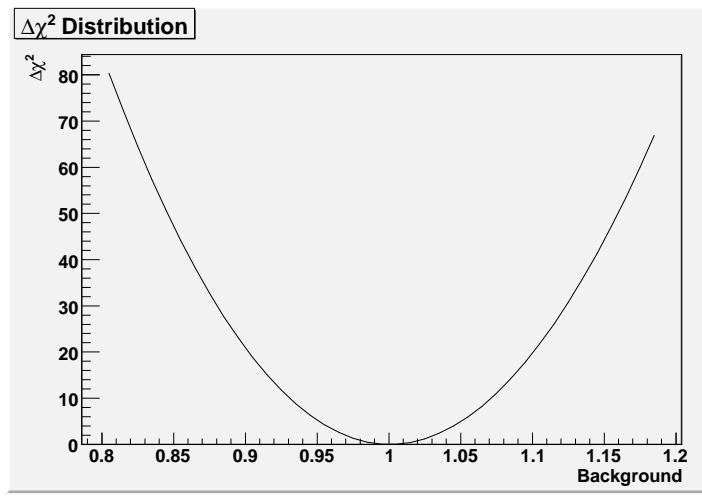

(c) NonQE-scale

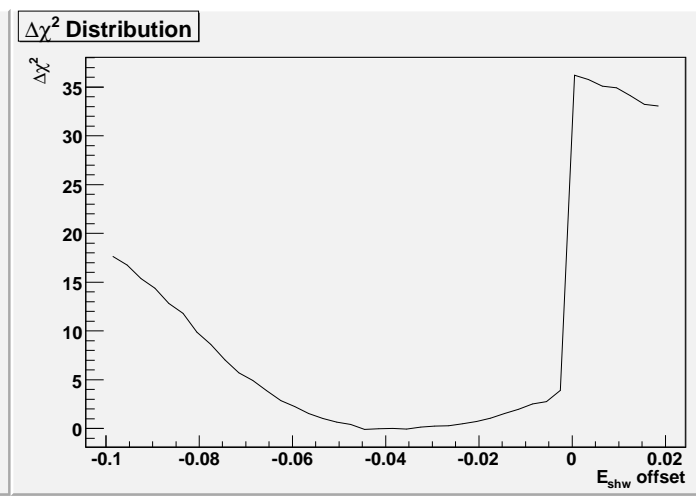

(b) $E_{s h w}$-offset

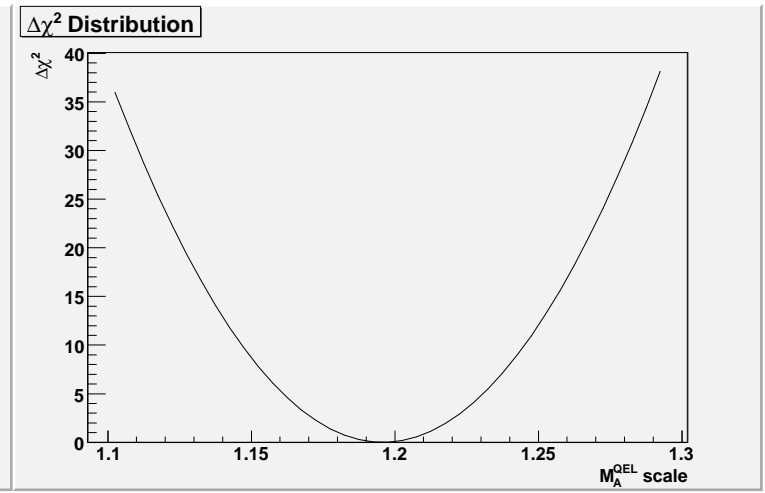

(d) $M_{A}^{Q E L}$-scale

FIG. 60: The parameter $\Delta \chi^{2}$ curves of the shape+rate fit to the mock data. 


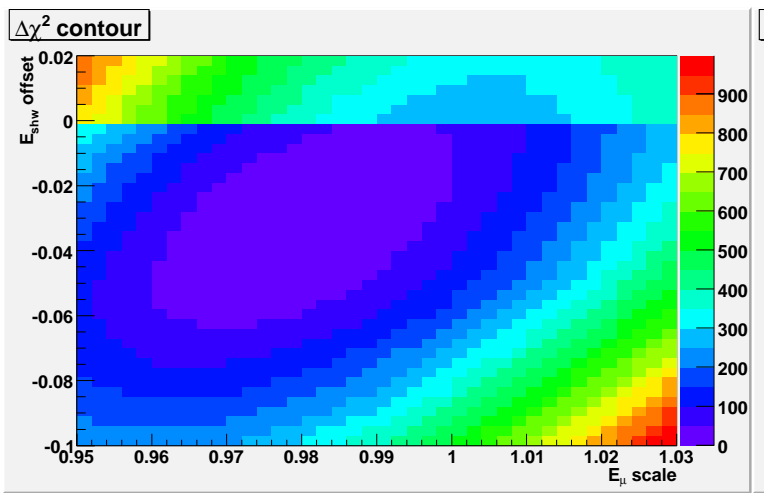

(a) $E_{\mu}$-scale vs $E_{s h w}$-offset

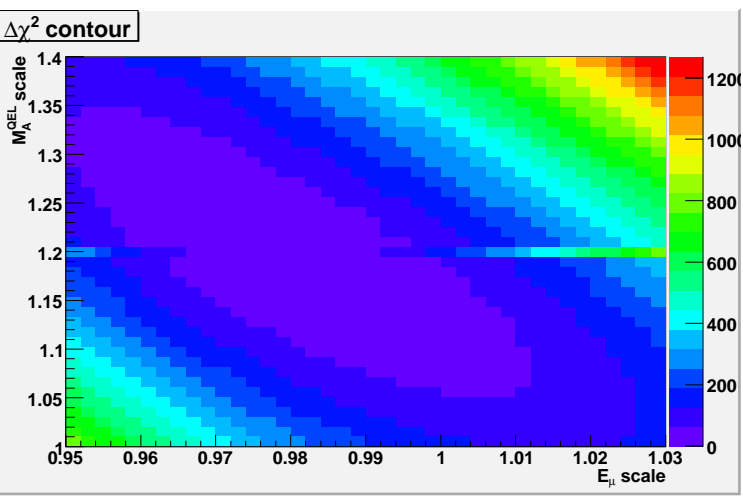

(b) $E_{\mu}$-scale vs $M_{A}^{Q E L}$-scale

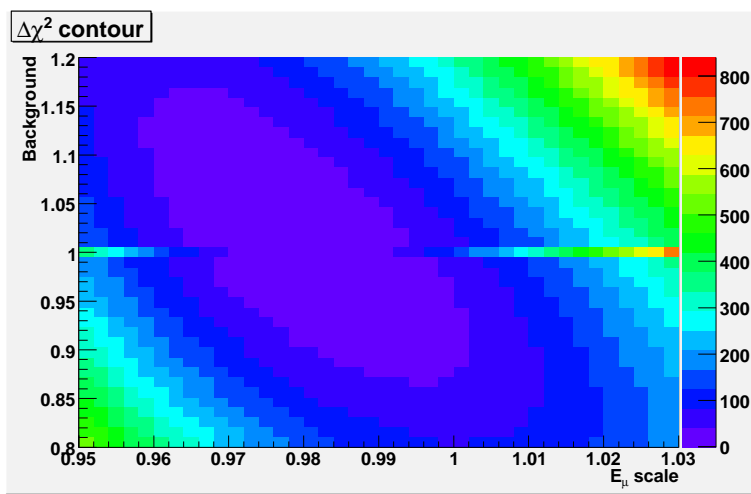

(c) $E_{\mu}$-scale vs $N o n Q E$-scale

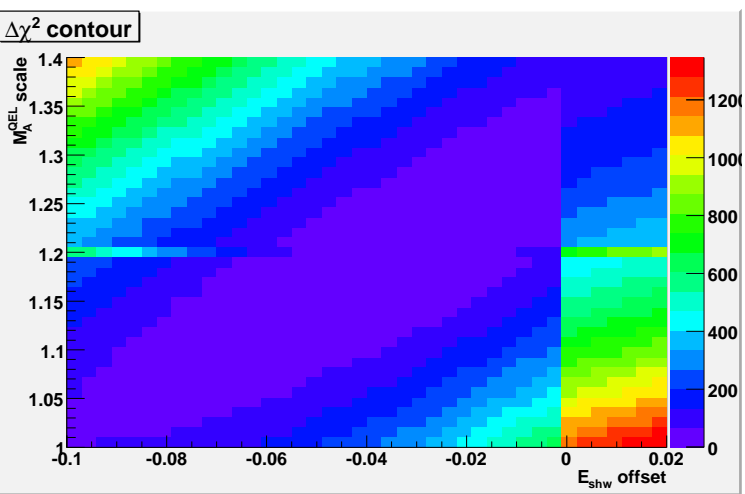

(d) $E_{s h w}$-offset vs $M_{A}^{Q E L}$-scale

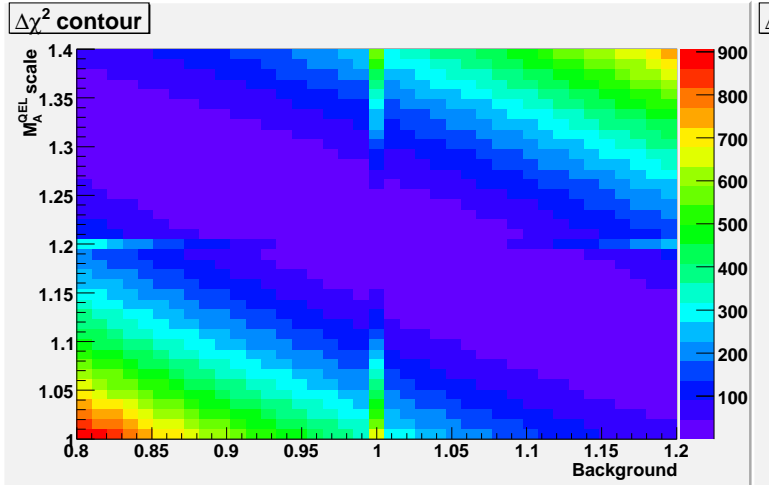

(e) NonQE-scale vs $M_{A}^{Q E L}$-scale

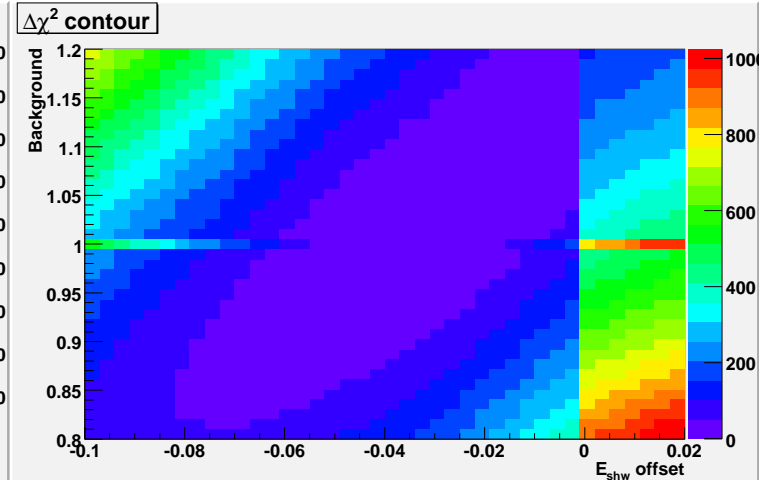

(f) $E_{s h w}$-offset vs $N o n Q E$-scale

FIG. 61: The parameter correlations of the shape+rate fit to the mock data. 
Data fit results : Data fit results are shown in Table V. (I) The shape-only fit result is shown in third column of the table. The best fit value is $M_{A}^{Q E L}=1.143 \mathrm{GeV}$ and doesn't agree to $\chi^{2}=42.768 / 16$. The $E_{\nu}$ and $Q^{2}$ distributions, and the parameter $\Delta \chi^{2}$ curves respect to the shape-only best fit value are shown in Figures 62 and 63 . Figure 64 shows the parameter correlations. (III) The shape+rate fit result is also shown in fifth column of the table. The best fit value is $M_{A}^{Q E L}=1.095 \mathrm{GeV}$ and doesn't agree to $\chi^{2}=163.328 / 37$. The $E_{\nu}$ and $Q^{2}$ distributions, and the parameter $\Delta \chi^{2}$ curves respect to the shape-only best fit value are shown in Figures 65 and 66 . Figure 67 shows the parameter correlations.

Furthermore, two more fit results are shown in the table. Single pion resonance production is one of the dominant background processes and well formulated with the $M_{A}^{R E S}$ scale in the NUEGEN event generator. Changing the $M_{A}^{R E S}$ scale largely influences the shape and normalization of the $Q^{2}$ distribution. For scaling the background spectrum in better shape, the $M_{A}^{R E S}$-scale is used in these additional fits, instead of the background scale. (II) The shape-only fit result is shown in fourth column of the table. The best fit value is $M_{A}^{Q E L}=1.223 \mathrm{GeV}$ and doesn't agree to $\chi^{2}=41.411 / 16$. The $E_{\nu}$ and $Q^{2}$ distributions, and the parameter $\Delta \chi^{2}$ curves respect to the shape-only best fit value are shown in Figures 68 and 69. Figure 70 shows the parameter correlations. (IV) The shape+rate fit result is also shown in sixth column of the table. The best fit value is $M_{A}^{Q E L}=1.196 \mathrm{GeV}$ and doesn't agree to $\chi^{2}=228.890 / 37$. The $E_{\nu}$ and $Q^{2}$ distributions, and the parameter $\Delta \chi^{2}$ curves respect to the shape-only best fit values are shown in Figures 71, and 72. Figure 73 shows the parameter correlations. 
Table V: Near Detector data fit results. Four parameter fits with NonQE scale are shown in 2nd and 4th columns. 3rd and 5th columns show four parameter fit results with the $M^{R E S}$-scale. The minimum values of the $\chi^{2}$ are larger than degree of freedom.

\begin{tabular}{|c|c|c|c|c|}
\hline & (I) & (II) & (III) & (IV) \\
\hline Fit configuration & \multicolumn{2}{|c|}{ shape-only fit } & \multicolumn{2}{|c|}{ rate+shape fit } \\
\hline$Q^{2}$ cut & \multicolumn{4}{|c|}{$Q^{2}>0.2 \mathrm{GeV}^{2}$} \\
\hline $\mathrm{MC}$ & \multicolumn{4}{|c|}{$1.47233 \times 10^{20}$ pot } \\
\hline Data & \multicolumn{4}{|c|}{$1.27000 \times 10^{20} \mathrm{pot}$} \\
\hline \multicolumn{5}{|c|}{ Fit Result } \\
\hline Obs. Events & \multicolumn{4}{|c|}{131505} \\
\hline Exp. Events & 131093 & 131089 & 131270 & 130968 \\
\hline Obs/Exp Events & 1.00314 & 1.00317 & 1.00179 & 1.00410 \\
\hline$E_{\mu}$ scale & 1.000 & 1.000 & 1.000 & 1.000 \\
\hline$E_{\text {shw }}$ scale & - & - & - & - \\
\hline$E_{s h w}$ offset $[\mathrm{GeV}]$ & -0.0614 & -0.0577 & -0.0634 & -0.0515 \\
\hline NonQE Background & 0.985 & - & 1.051 & - \\
\hline$M_{A}^{Q E L}$ scale & 1.132 & 1.211 & 1.084 & 1.184 \\
\hline$M_{A}^{R E S}$ scale & - & 0.874 & - & 0.944 \\
\hline$R E S / D I S$ scale & - & - & - & - \\
\hline$\chi^{2}\left(E_{\nu}\right)$ & $N / A$ & $\mathrm{~N} / \mathrm{A}$ & 119.708 & 167.016 \\
\hline$\chi^{2}\left(Q^{2}\right)$ & 41.1724 & 39.377 & 40.986 & 60.674 \\
\hline$\chi^{2}$ (syst.) & 1.596 & 2.033 & 2.634 & 1.201 \\
\hline$\chi^{2}($ total $) / d o f$ & $42.768 / 16$ & $41.411 / 16$ & $163.328 / 37$ & $228.890 / 37$ \\
\hline
\end{tabular}




\section{— Data Fit Result (I) -}
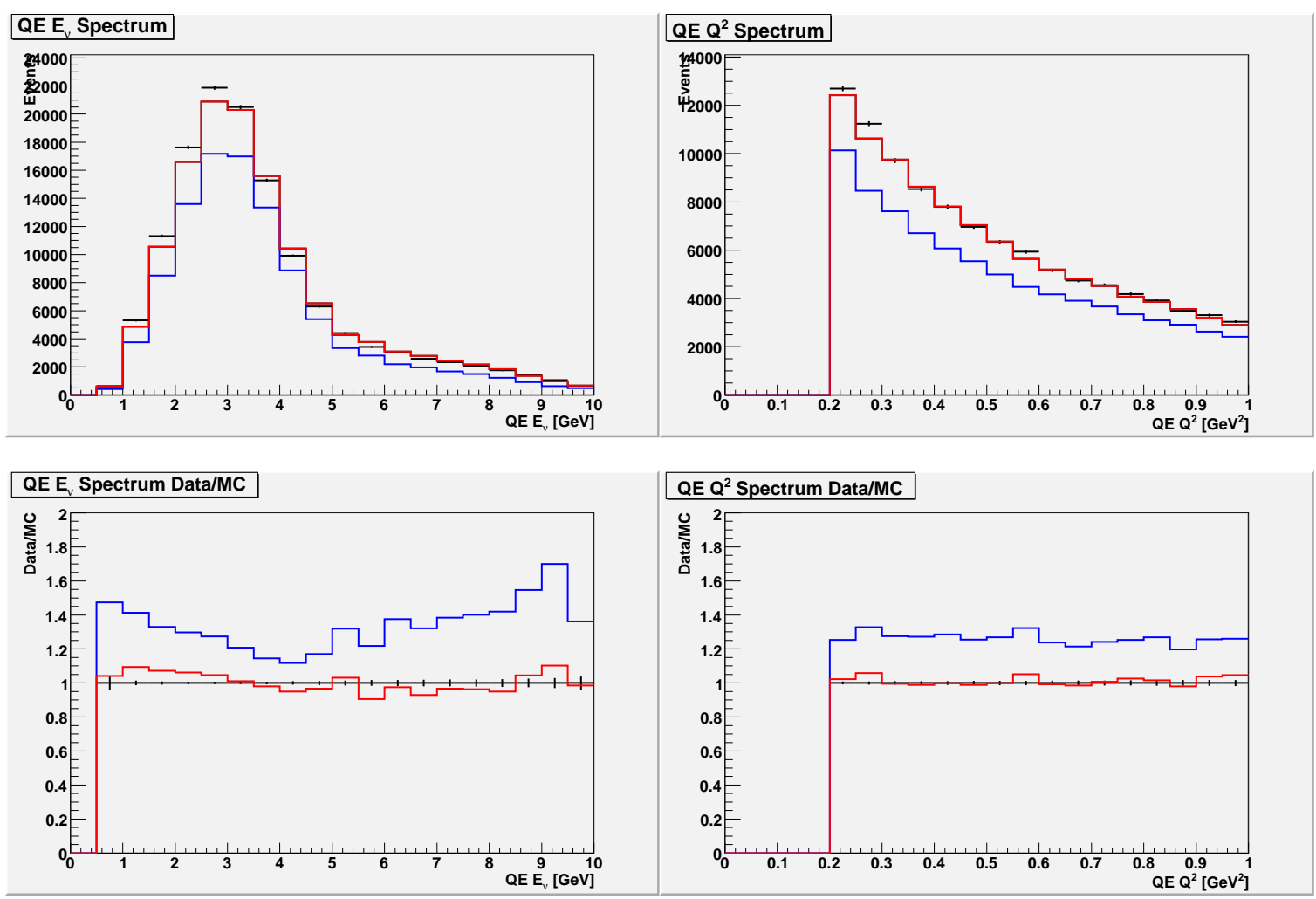

FIG. 62: Fit result (I) : the $E_{\nu}$ and $Q^{2}$ distributions and data/MC ratios of the shape-only fit to the data. Blue and red lines show nominal and best fit spectra. 


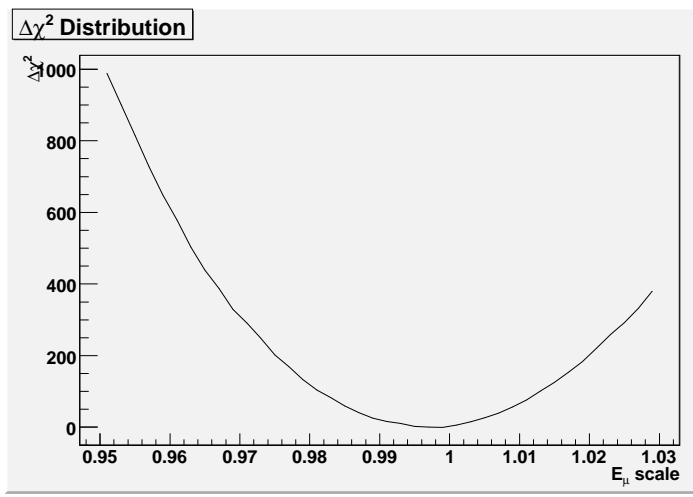

(a) $E_{\mu}$-scale

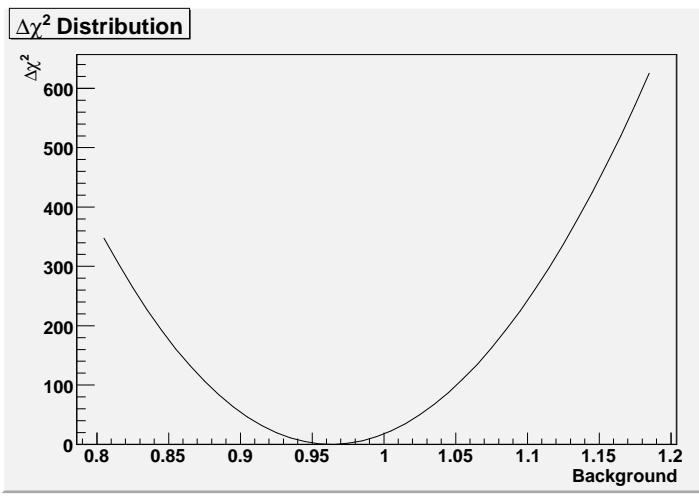

(c) NonQE-scale

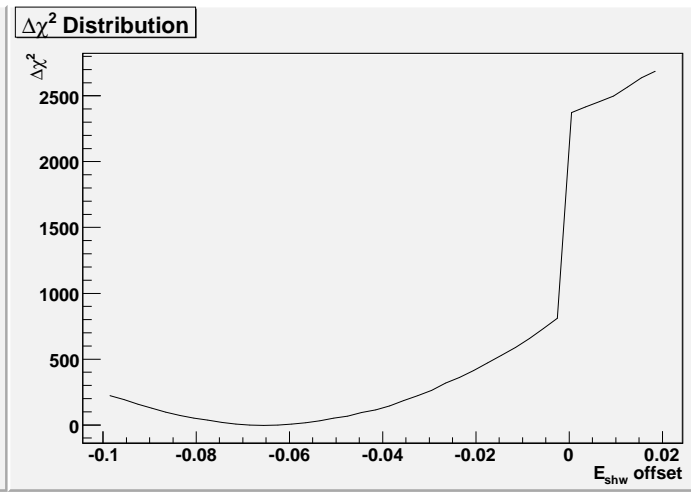

(b) $E_{s h w}$-offset

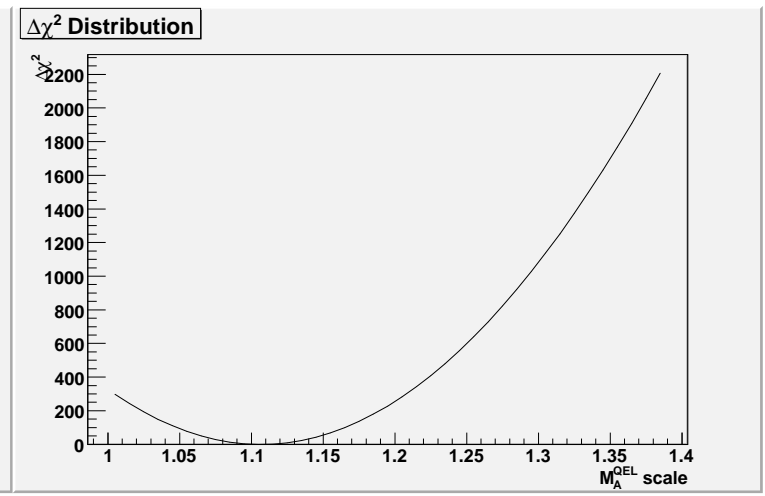

(d) $M_{A}^{Q E L}$-scale

FIG. 63: Fit result (I) : the parameter $\Delta \chi^{2}$ curves of the shape-only fit to the data. 


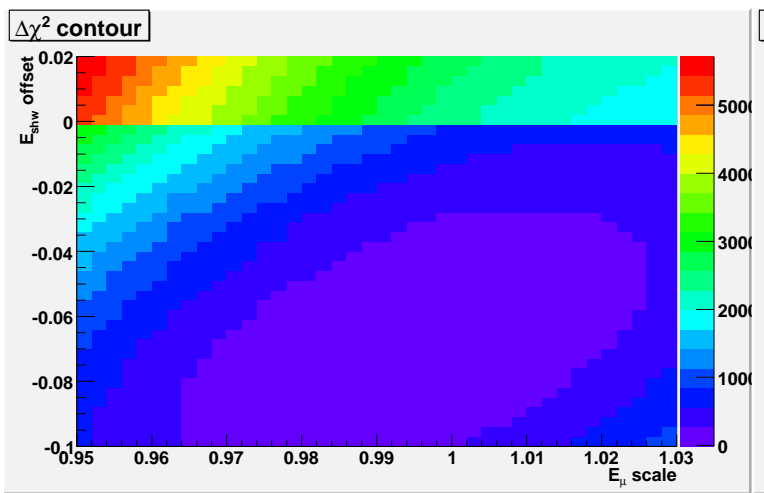

(a) $E_{\mu}$-scale vs $E_{s h w}$-offset

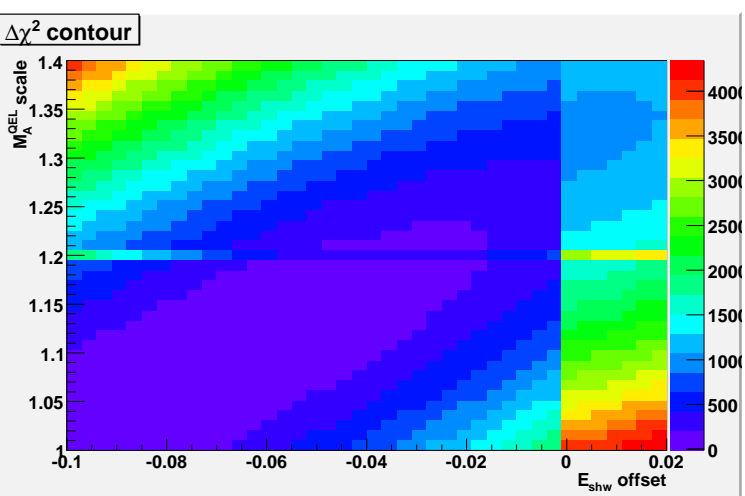

(b) $E_{\text {shw-offset vs }} M_{A}^{Q E L}$-scale

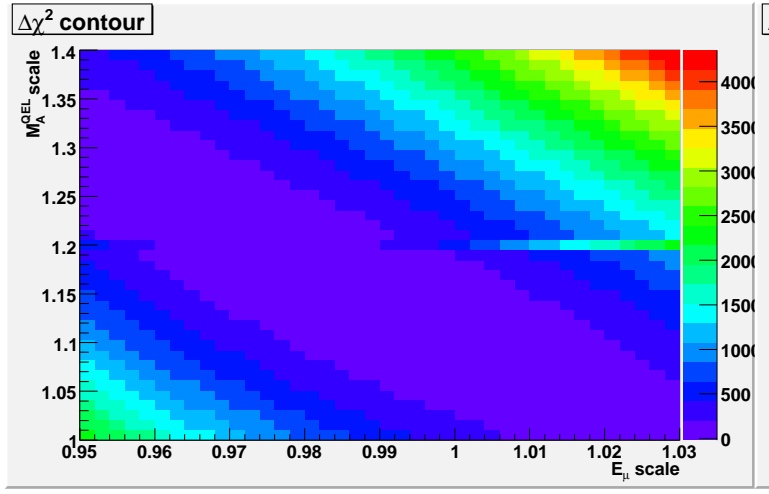

(c) $E_{\mu}$-scale vs $M_{A}^{Q E L}$-scale

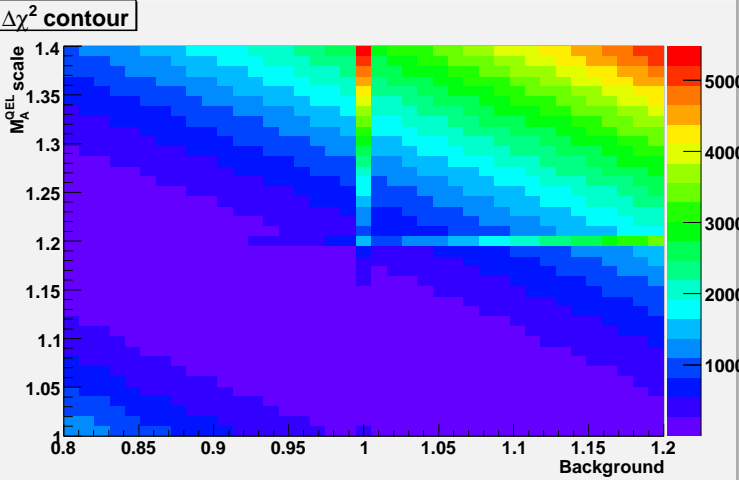

(d) NonQE-scale vs $M_{A}^{Q E L}$-scale

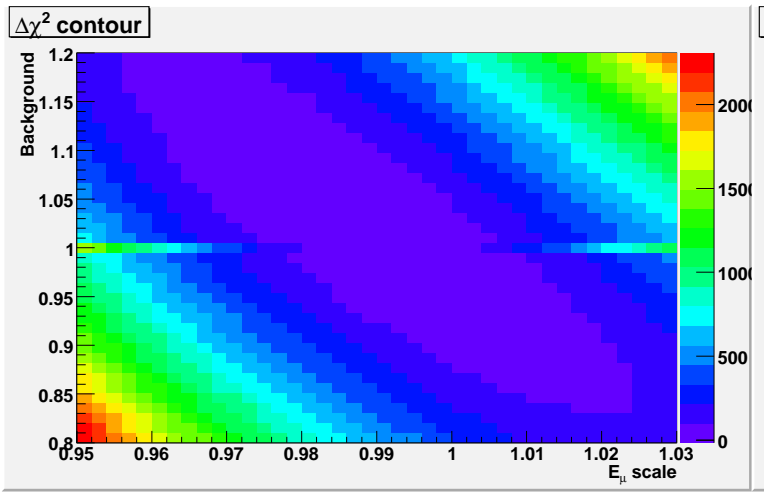

(e) $E_{\mu}$-scale vs $N o n Q E$-scale

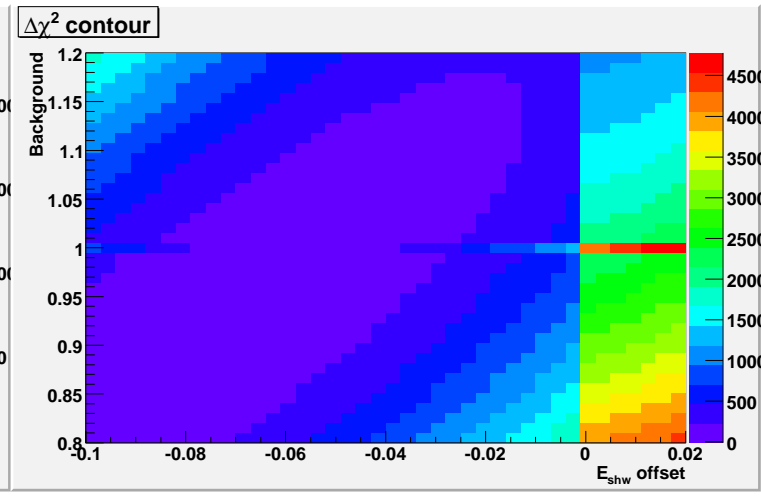

(f) $E_{s h w}$-offset vs $N o n Q E$-scale

FIG. 64: Fit result (I) : the parameter correlation for the shape-only fit to the data. 


\section{— Data Fit Result (III) -}
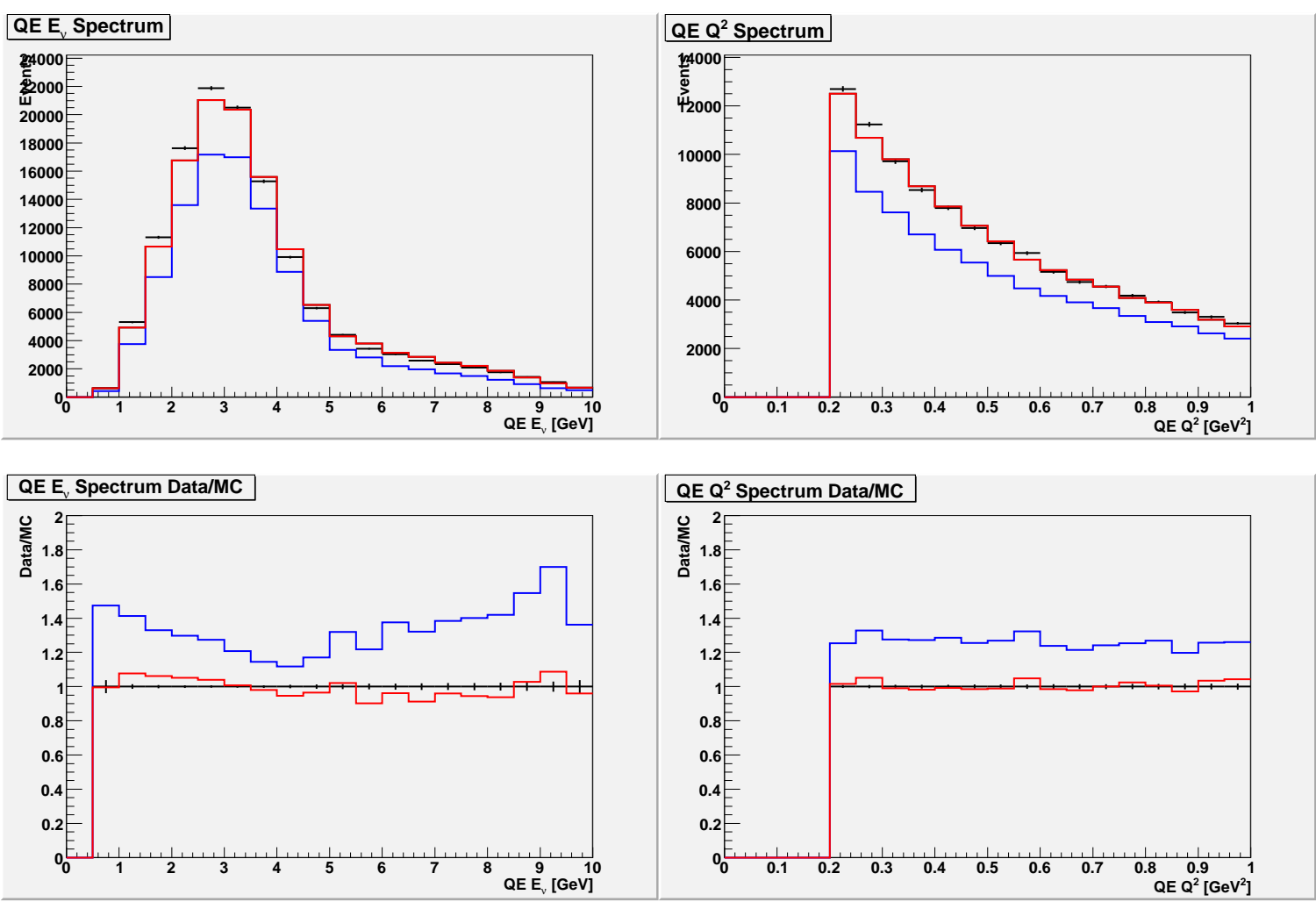

FIG. 65: Fit result (III) : the $E_{\nu}$ and $Q^{2}$ distributions and data/MC ratios of the shape+rate fit to the data. Blue and red lines are for the nominal and best fit spectra. 


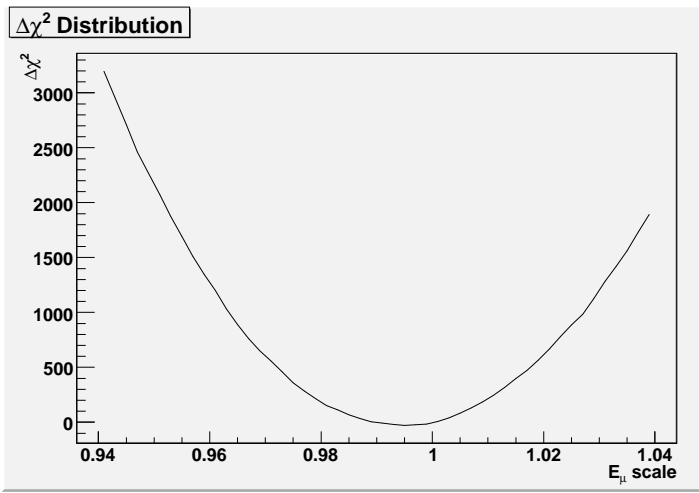

(a) $E_{\mu}$-scale

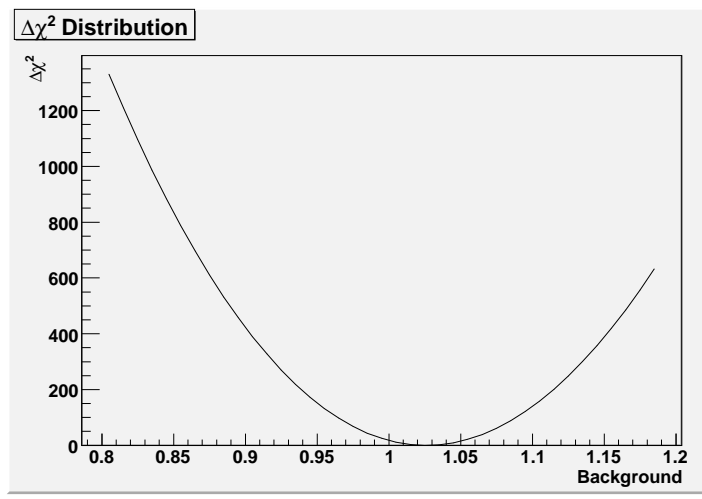

(c) NonQE-scale

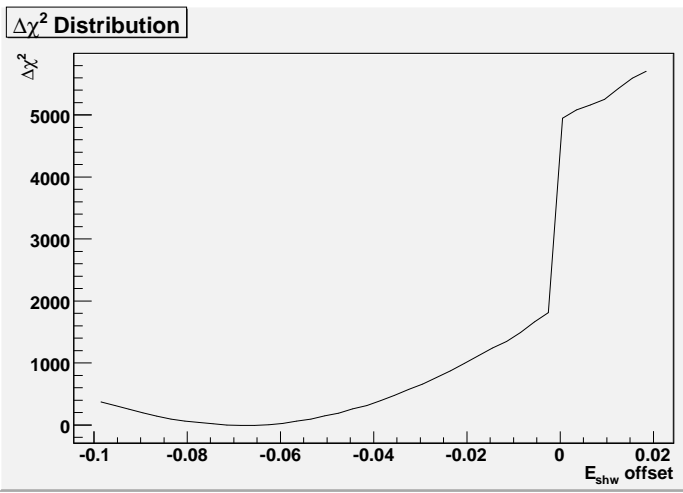

(b) $E_{s h w}$-offset

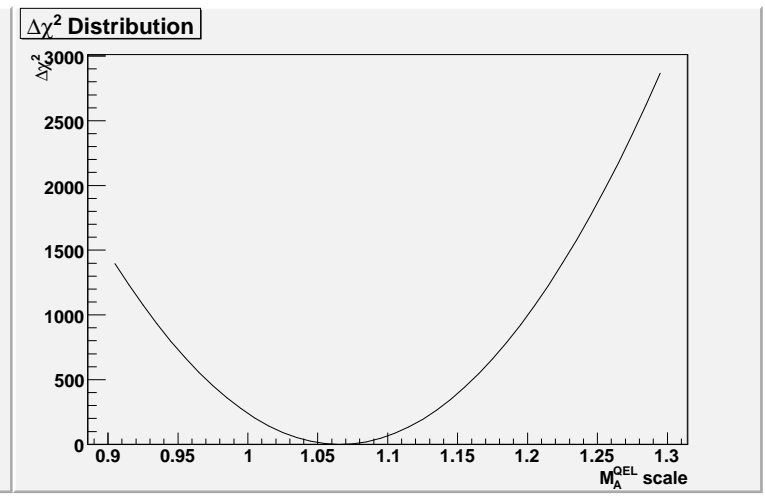

(d) $M_{A}^{Q E L}$-scale

FIG. 66: Fit result (III) : the parameter $\Delta \chi^{2}$ curves of the shape+rate fit to the data. 


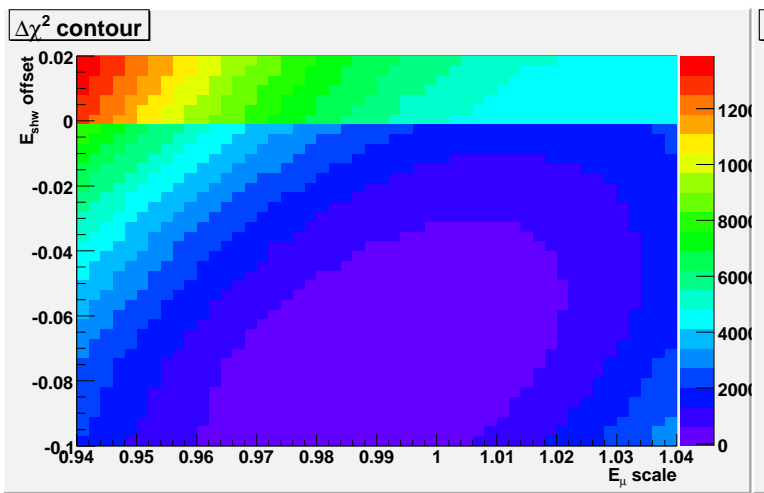

(a) $E_{\mu}$-scale vs $E_{s h w}$-offset

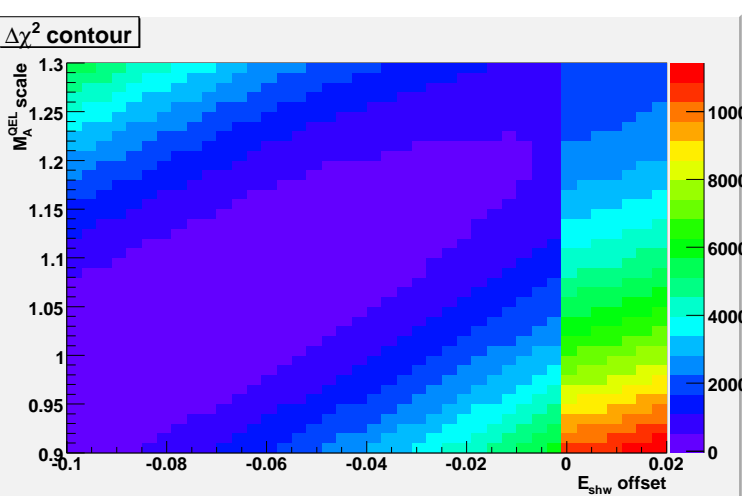

(b) $E_{s h w}$-offset vs $M_{A}^{Q E L}$-scale

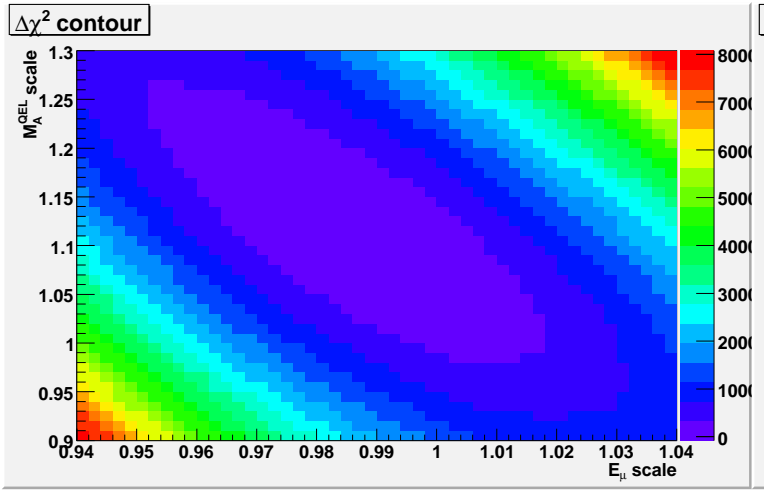

(c) $E_{\mu}$-scale vs $M_{A}^{Q E L}$-scale

\section{$\Delta \chi^{2}$ contour}

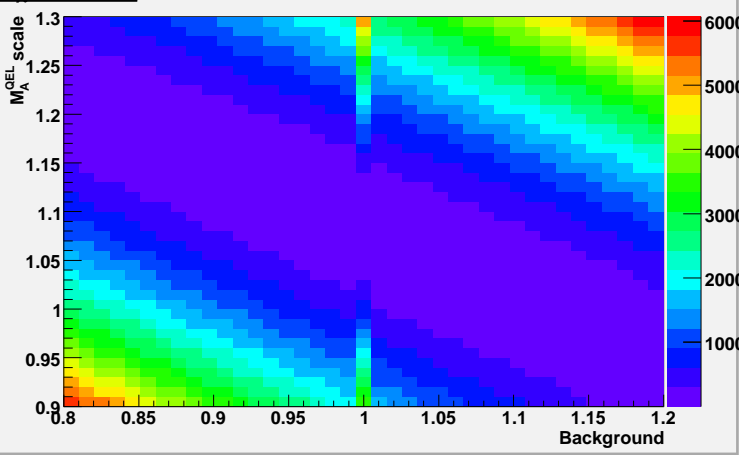

(d) $M_{A}^{Q E L}$-scale vs $N o n Q E$-scale

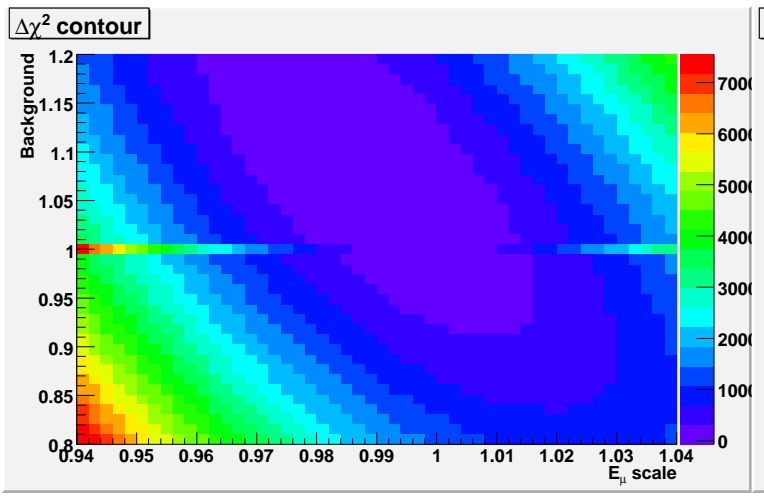

(e) $E_{\mu}$-scale vs $N o n Q E$-scale

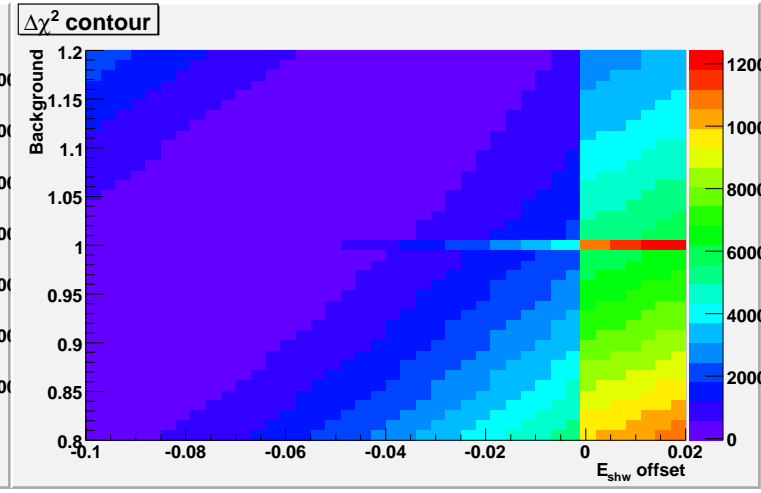

(f) $E_{\text {shw }}$-offset vs $N o n Q E$-scale

FIG. 67: Fit result (III) : the parameter correlation for the shape+rate fit to the data. 


\section{— Data Fit Result (II) -}
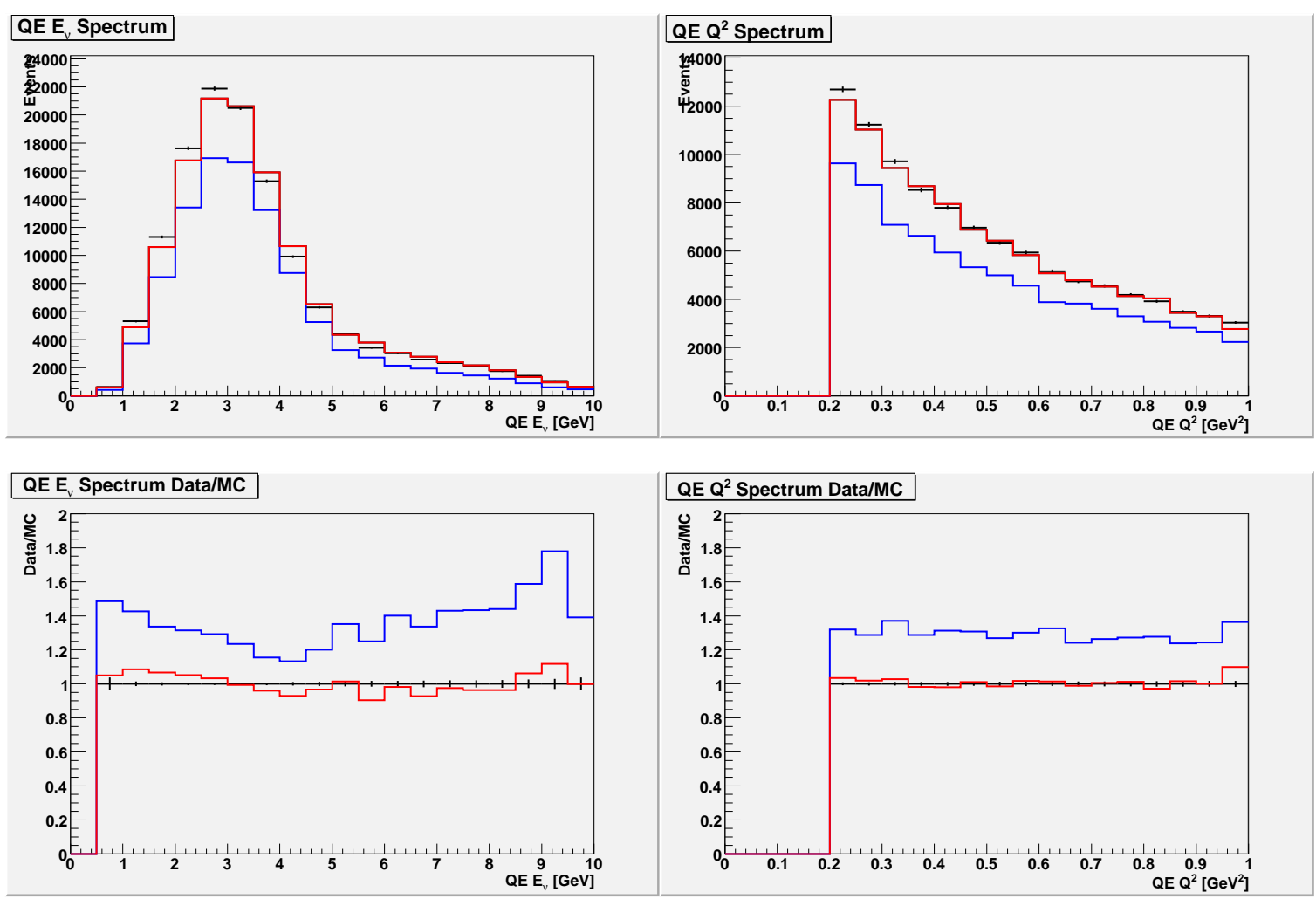

FIG. 68: Fit result (II) : the $E_{\nu}$ and $Q^{2}$ distributions and data/MC ratios of the shape-only fit to the data. Blue and red lines are for the nominal and best fit spectra. 


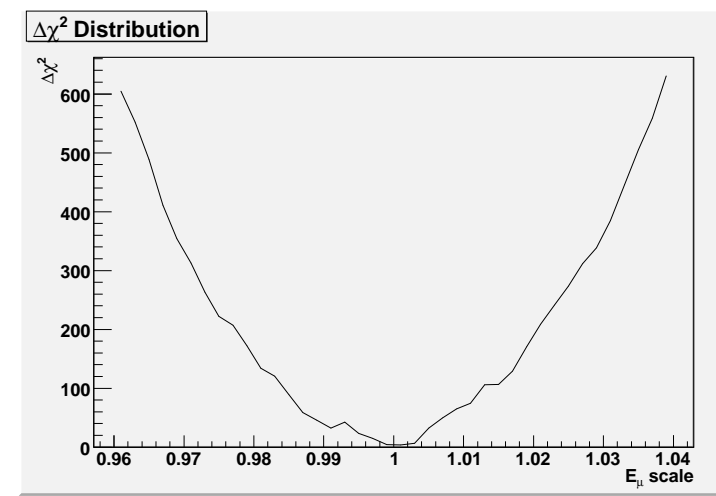

(a) $E_{\mu}$-scale

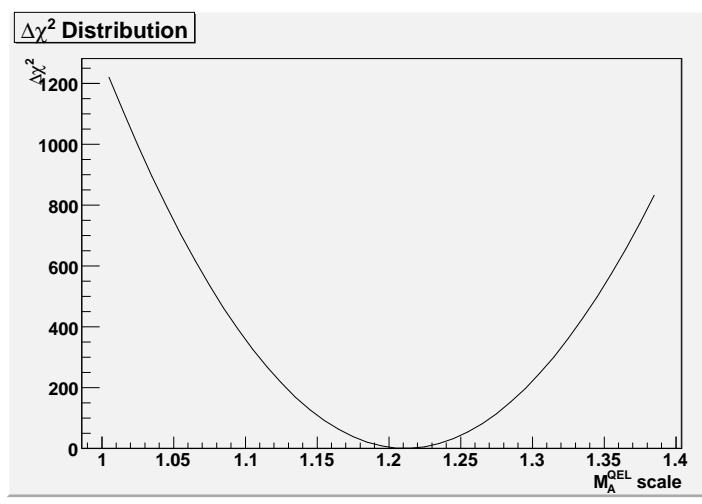

(c) $M_{A}^{Q E L}$-scale

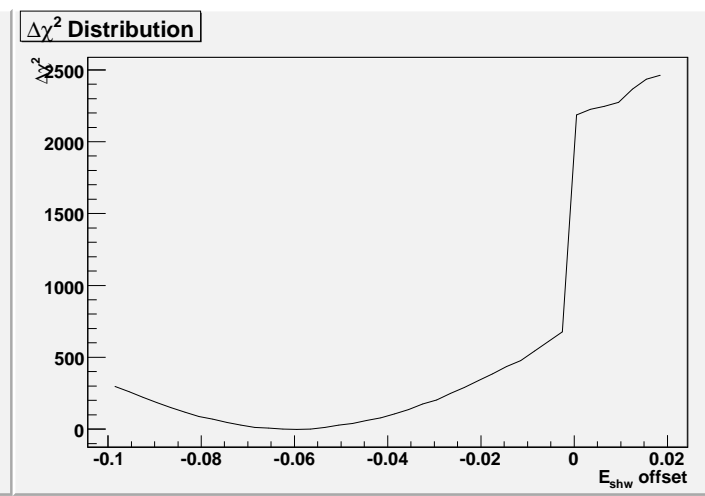

(b) $E_{s h w}$-offset

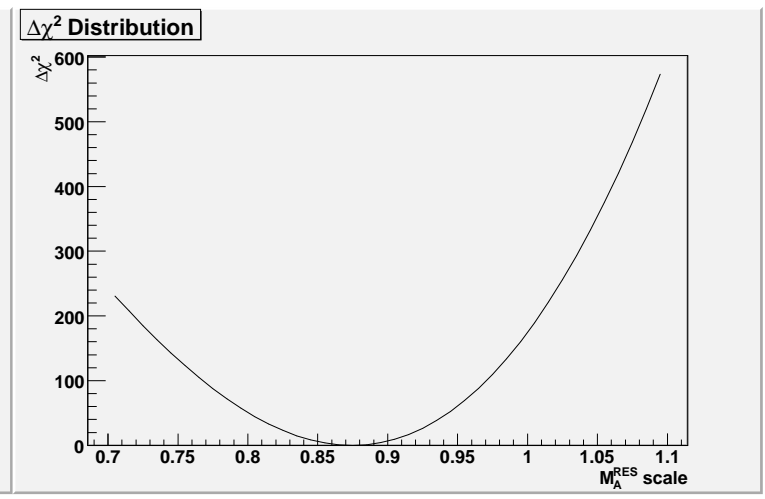

(d) $M_{A}^{R E S}$-scale

FIG. 69: Fit result (II) : the parameter $\Delta \chi^{2}$ curves of the shape-only fit to the data. 


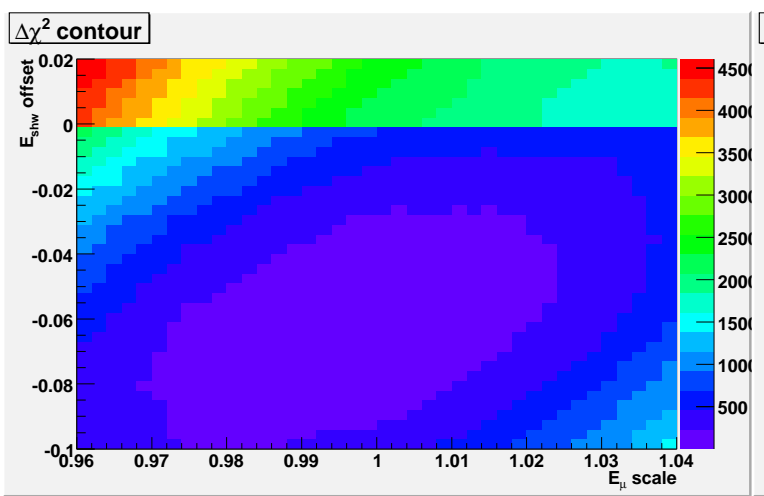

(a) $E_{\mu}$-scale vs $E_{s h w}$-offset

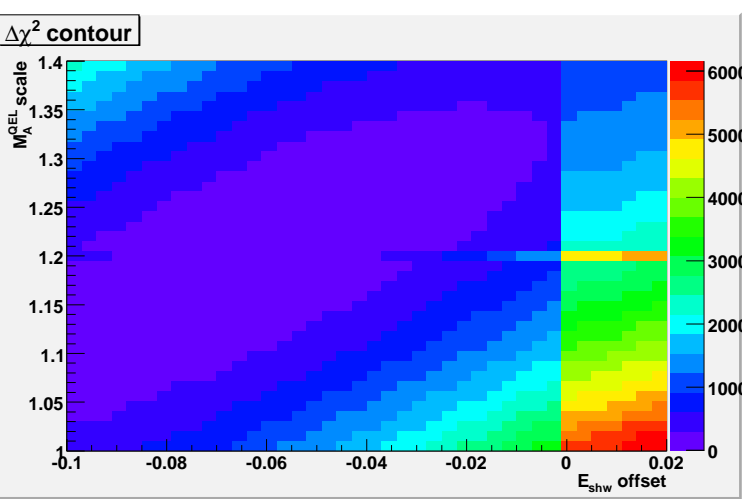

(b) $E_{s h w}$-offset vs $M_{A}^{Q E L}$-scale

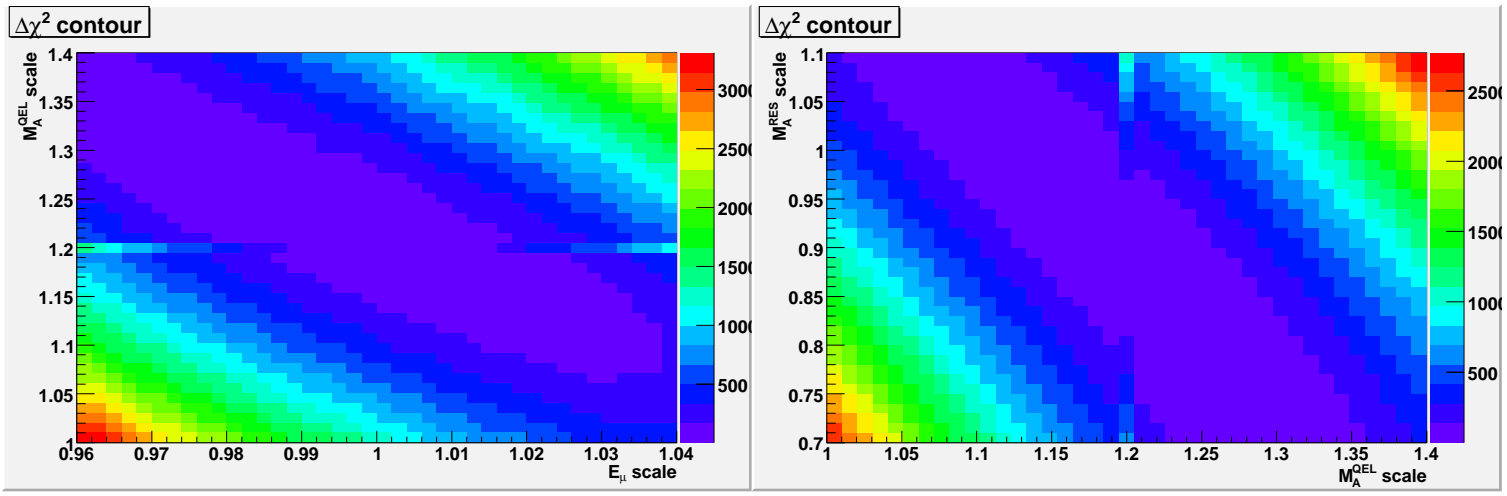

(c) $E_{\mu}$-scale vs $M_{A}^{Q E L}$-scale

(d) $M_{A}^{Q E L}$-scale vs $M_{A}^{R E S_{\text {-scale }}}$

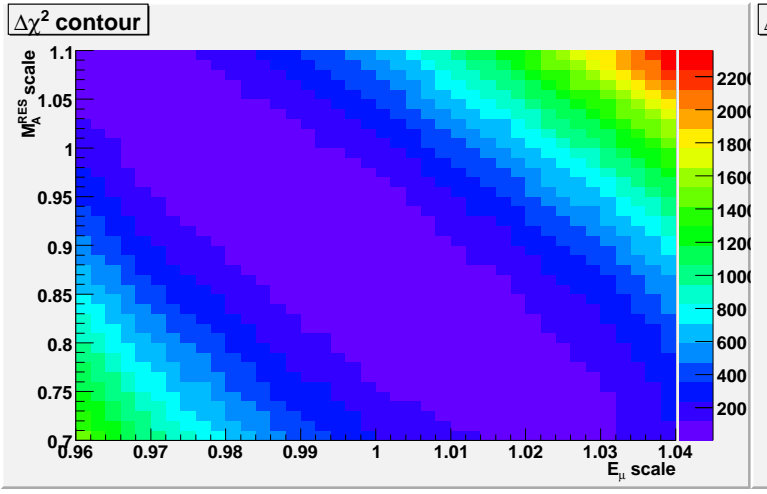

(e) $E_{\mu}$-scale vs $M_{A}^{R E S}$-scale

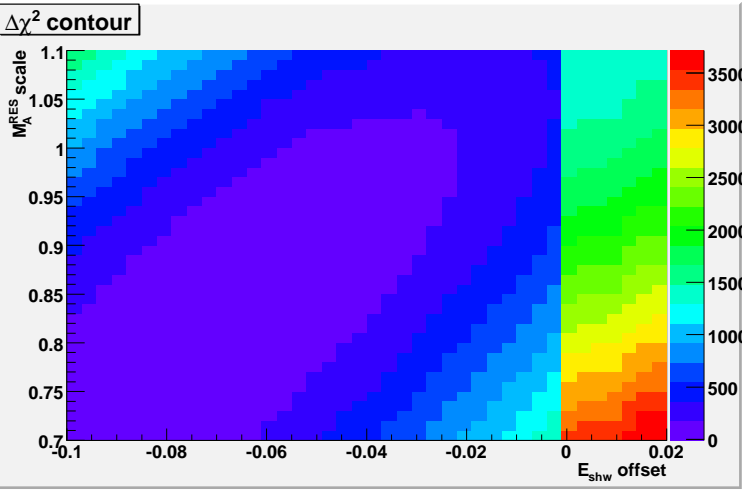

(f) $E_{\text {shw }}$-offset vs $M_{A}^{R E S}$-scale

FIG. 70: Fit result (II) : the parameter correlation for the shape-only fit to the data. 


\section{— Data Fit Result (IV) -}
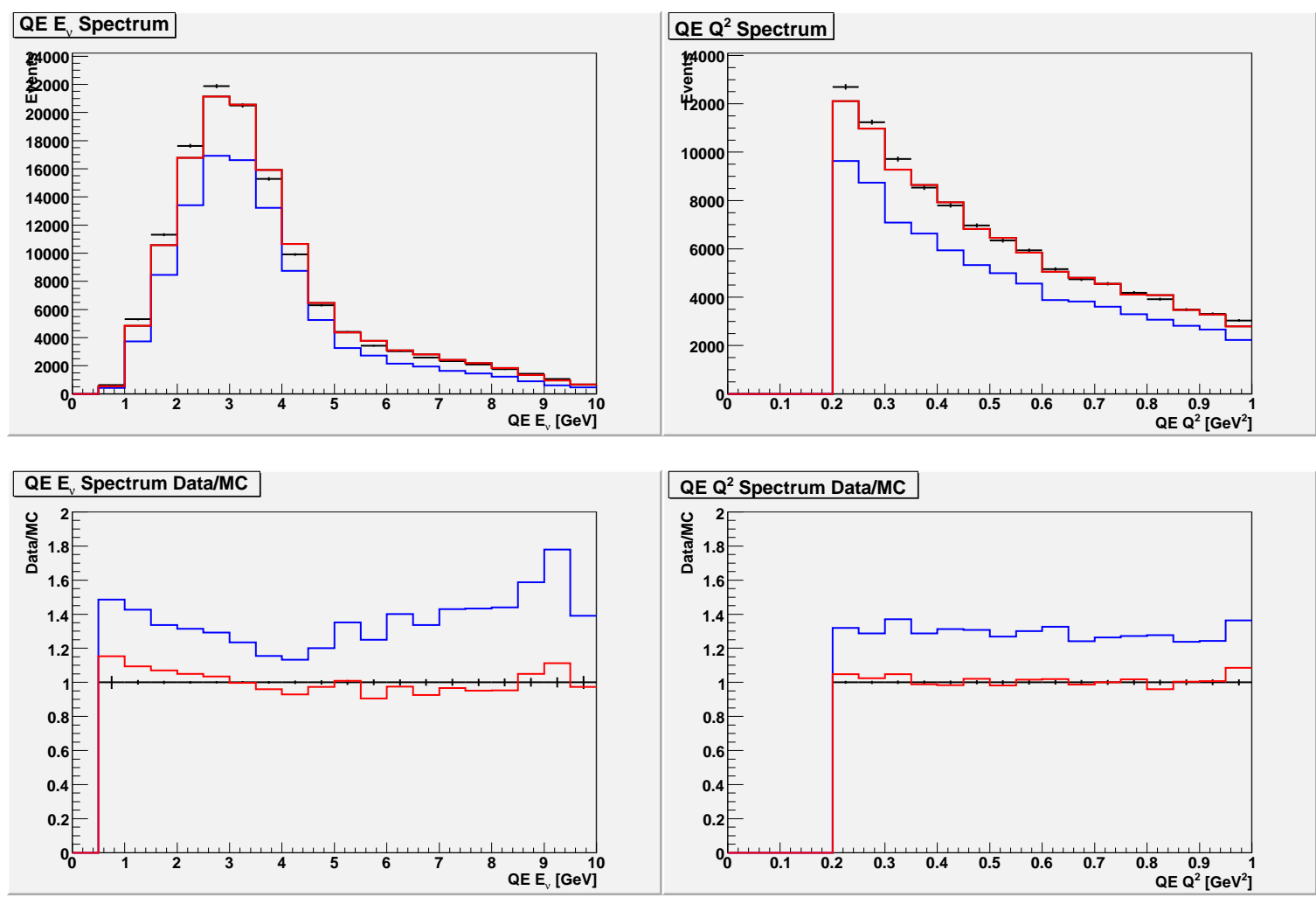

FIG. 71: Fit result (IV) : the $E_{\nu}$ and $Q^{2}$ distributions and data/MC ratios of the shape+rate fit to the data. Blue and red lines are for the nominal and best fit spectra. 


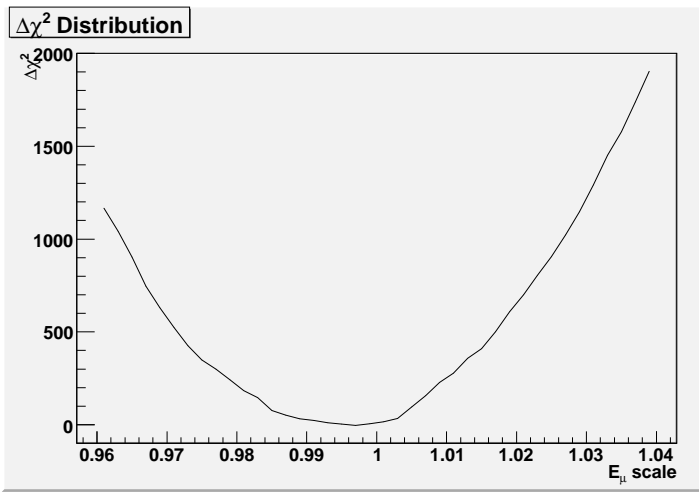

(a) $E_{\mu}$-scale

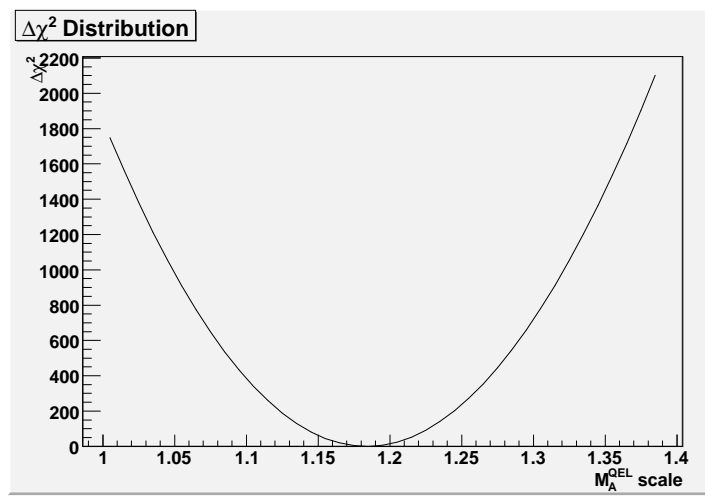

(c) $M_{A}^{Q E L}$-scale

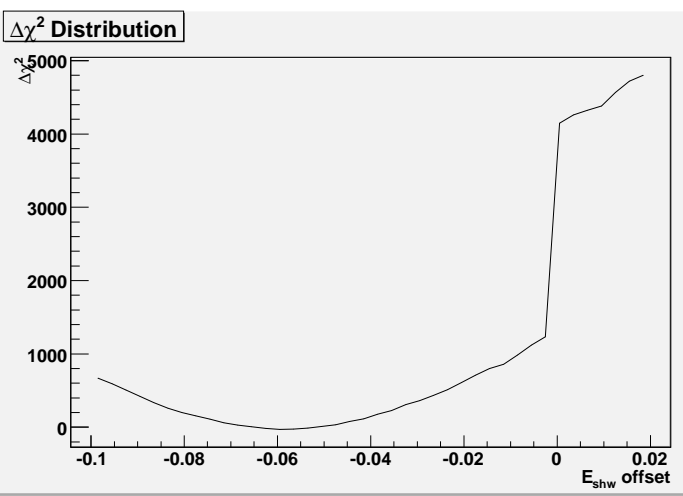

(b) $E_{s h w}$-offset

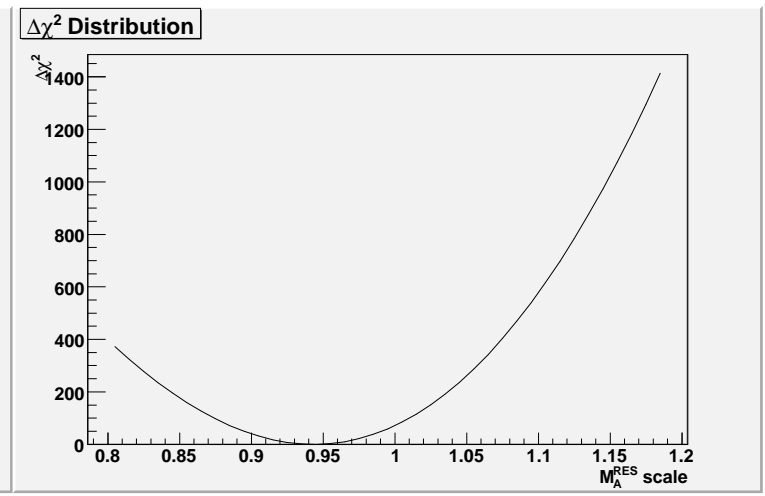

(d) $M_{A}^{R E S}$-scale

FIG. 72: Fit result (IV) : the parameter $\Delta \chi^{2}$ curves of the shape+rate fit to the data. 


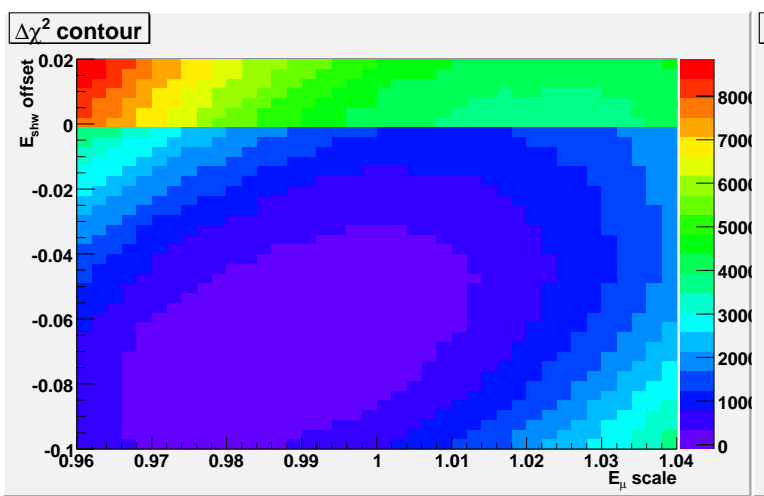

(a) $E_{\mu}$-scale vs $E_{s h w}$-offset

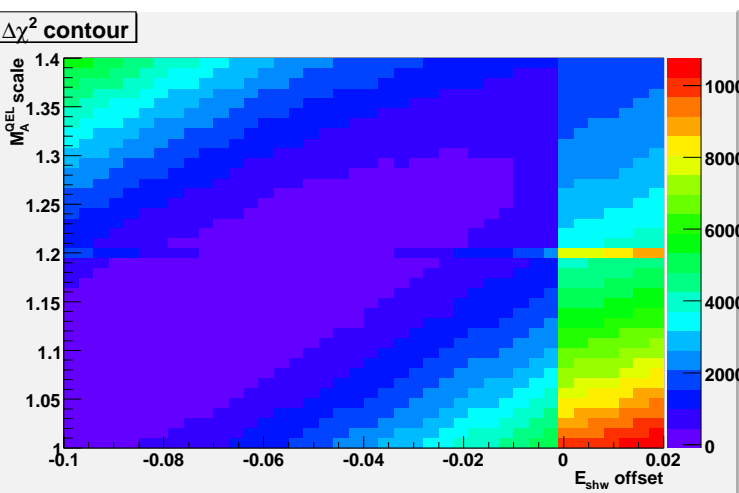

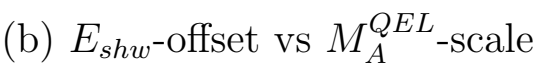

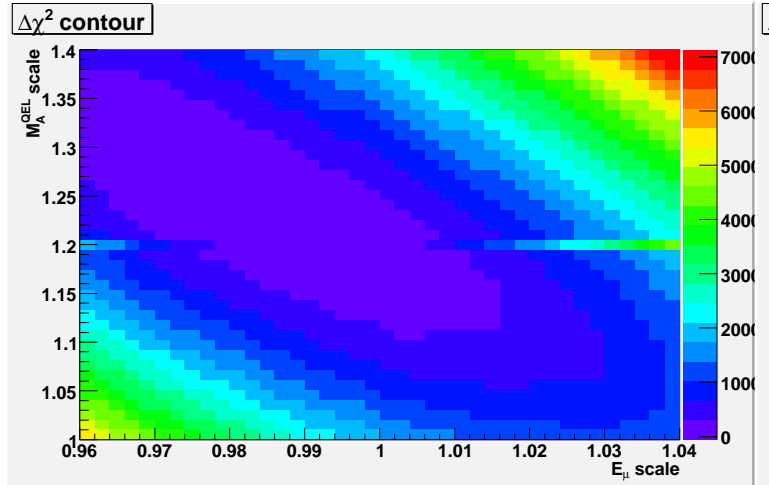

(c) $E_{\mu}$-scale vs $M_{A}^{Q E L}$-scale

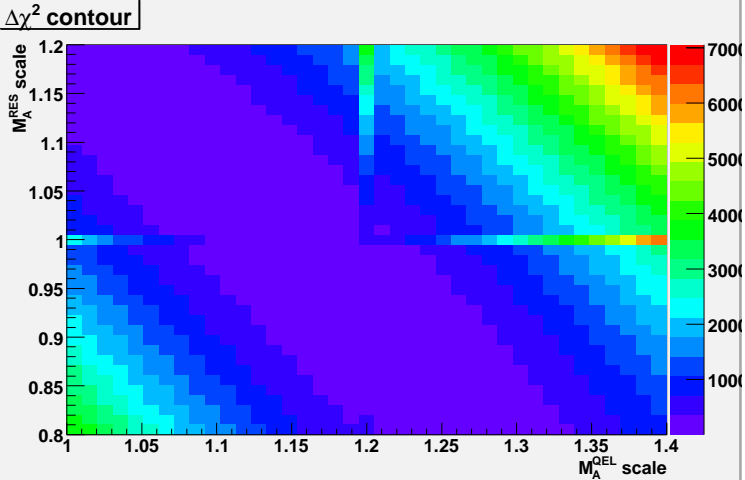

(d) $M_{A}^{Q E L}$-scale vs $M_{A}^{R E S}$-scale

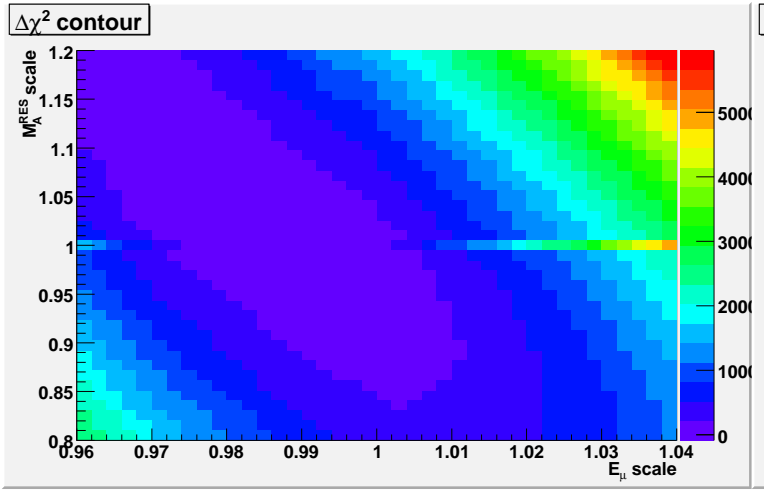

(e) $E_{\mu}$-scale vs $M_{A}^{R E S}$-scale

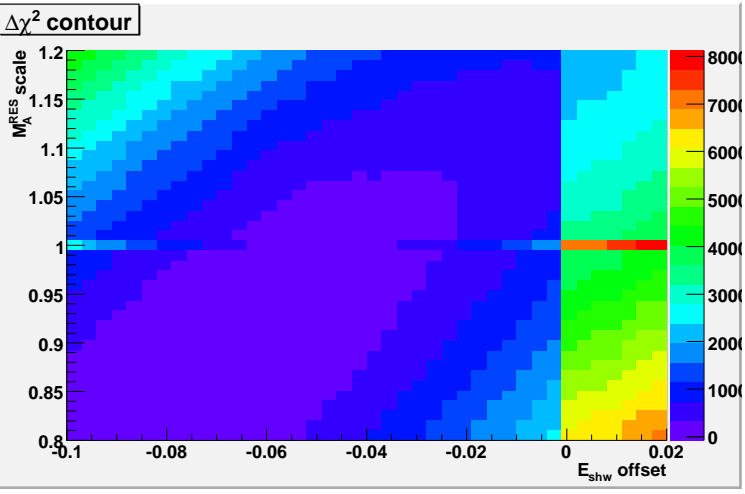

(f) $E_{\text {shw }}$-offset vs $M_{A}^{R E S}$-scale

FIG. 73: Fit result (IV) : the parameter correlation for the shape+rate fit to the data. 


\section{APPENDIX E}

\section{OSCILLATION FIT}

FD oscillation fit is tested for the far mock data set and constrained only in physical region of $\sin ^{2}(2 \theta) \leq 1.0$. The mock data contains $65 \times 10^{20}$ POT. It is generated with the detector and cross section parameters, SKZP reweighted beam spectrum and the oscillation parameters of $\sin ^{2}(2 \theta)=0.950$ and $\Delta m^{2}=2.500 \times 10^{-3} \mathrm{eV}^{2}$. The fit results are shown in Table VI.

Table VI: Far Detector oscillation mock data fit result. FD oscillation fit result for $65 \times 10^{20}$ pot mock data set using the shape+rate Near Detector fit result.

\begin{tabular}{|c|c|c|}
\hline & True & Best Fit \\
\hline $\sin ^{2}(2 \theta)$ & 0.950 & $0.931 \pm 0.0207$ \\
\hline$\Delta m^{2}\left(\times 10^{-3}\right) \mathrm{eV}^{2}$ & 2.500 & $2.541 \pm 0.0960$ \\
\hline \multicolumn{2}{|c|}{ obs/exp events } & $1263.85 / 2178.44$ \\
\hline \hline \multicolumn{2}{|c|}{$\chi^{2} /$ dof } & $10.971 / 9$ \\
\hline
\end{tabular}



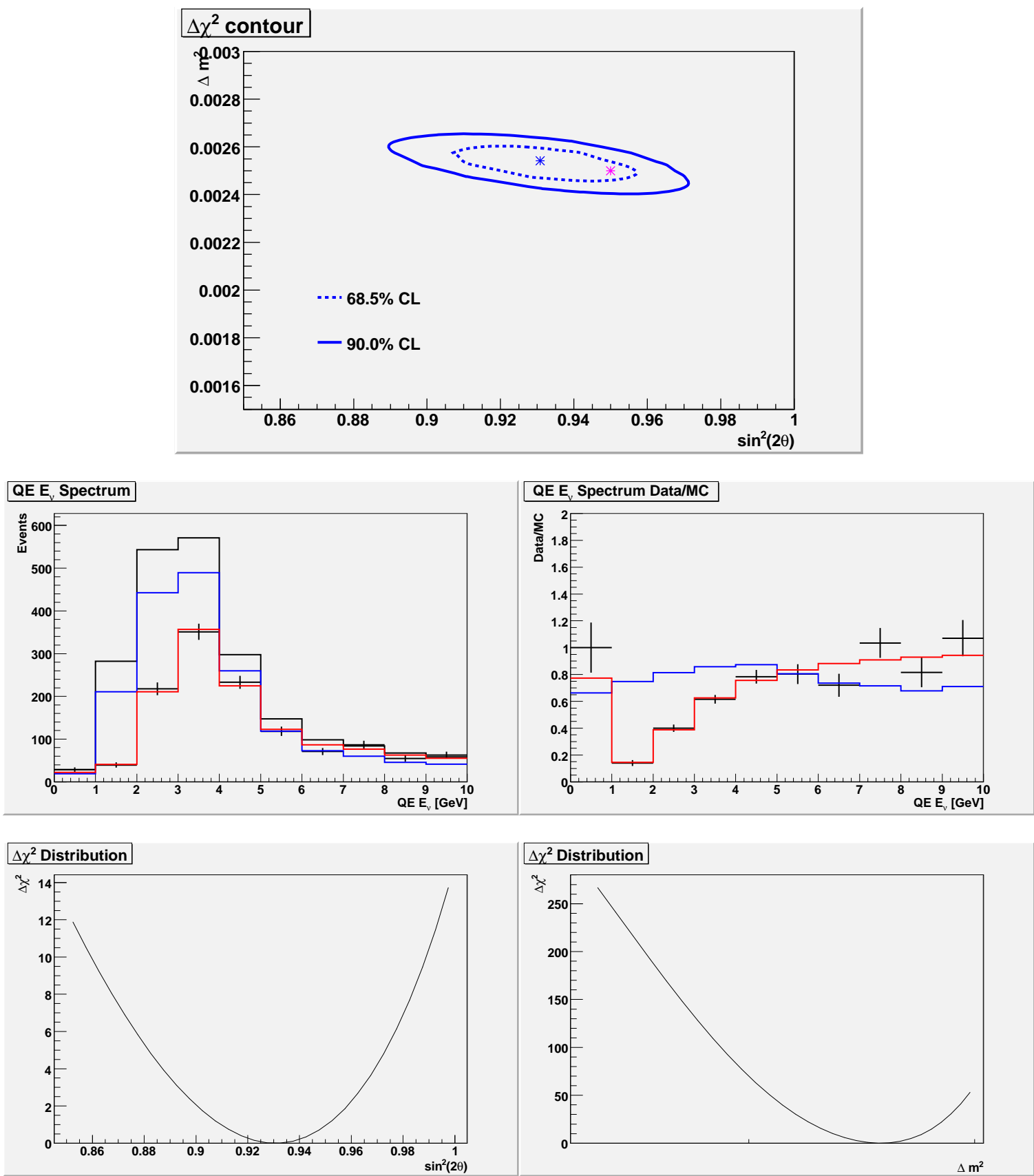

FIG. 74: The oscillation contour, $E_{\nu}$ distribution and data/MC ratio, and $\Delta \chi^{2}$ curves of the oscillation fit to the mock data. The oscillation contour of the oscillation fit to the mock data is shown in the top figure. Pink point indicates true values of oscillation parameters. The $E_{\nu}$ distribution and data/MC ratio of the oscillation fit to the mock data are shown in the middle left and right figures. The $\Delta \chi^{2}$ curves for the $\Delta m^{2}$ and mixing angle are shown in the bottom left and right figures. 


\section{VITA}

Name :

Address :

E-Mail Address :

Education :
B.S. $\quad$ May 2001

M.S. December 2005

Ph.D. $\quad$ May 2010

Masaki Watabe

Physics Department

Texas A\&M University

College Station, TX 77843-4242

mwatabe@neo.tamu.edu 